\author{
EXAMINING TEACHERS'PARADIGM AND PEDAGOGY \\ IN MANIFESTING CRITICAL HOPE IN LOW-INCOME \\ MIDDLE SCHOOL CLASSROOM:
}

A NARRATIVE INQUIRY

\author{
A DISSERTATION SUBMITTED TO THE SCHOOL OF EDUCATION OF THE \\ UNIVERSITY OF REDLANDS IN PARTIAL FULFILLMENT OF THE REQUIREMENTS \\ FOR THE DEGREE OF DOCTORATE OF EDUCATION IN LEADERSHIPFOR \\ EDUCATIONAL JUSTICE
}

\author{
Heidi Strikwerda \\ University of Redlands \\ Committee Members \\ Jose W. Lalas, Ph.D., Committee Chair \\ Barbra Flores, Ph.D. \\ Brian Charest, Ph.D.
}

August 2019 
EXAMINING TEACHERS' PARADIGM AND PEDAGOGY IN MANIFESTING CRITICAL

HOPE IN LOW-INCOME MIDDLE SCHOOL 


\section{Redilands \\ SCHOOL OF EDUCATIUN}

We hereby approve the dissertation of

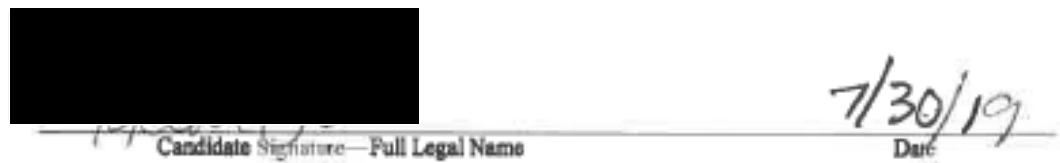

Candidate for the degree of Doctor of Education
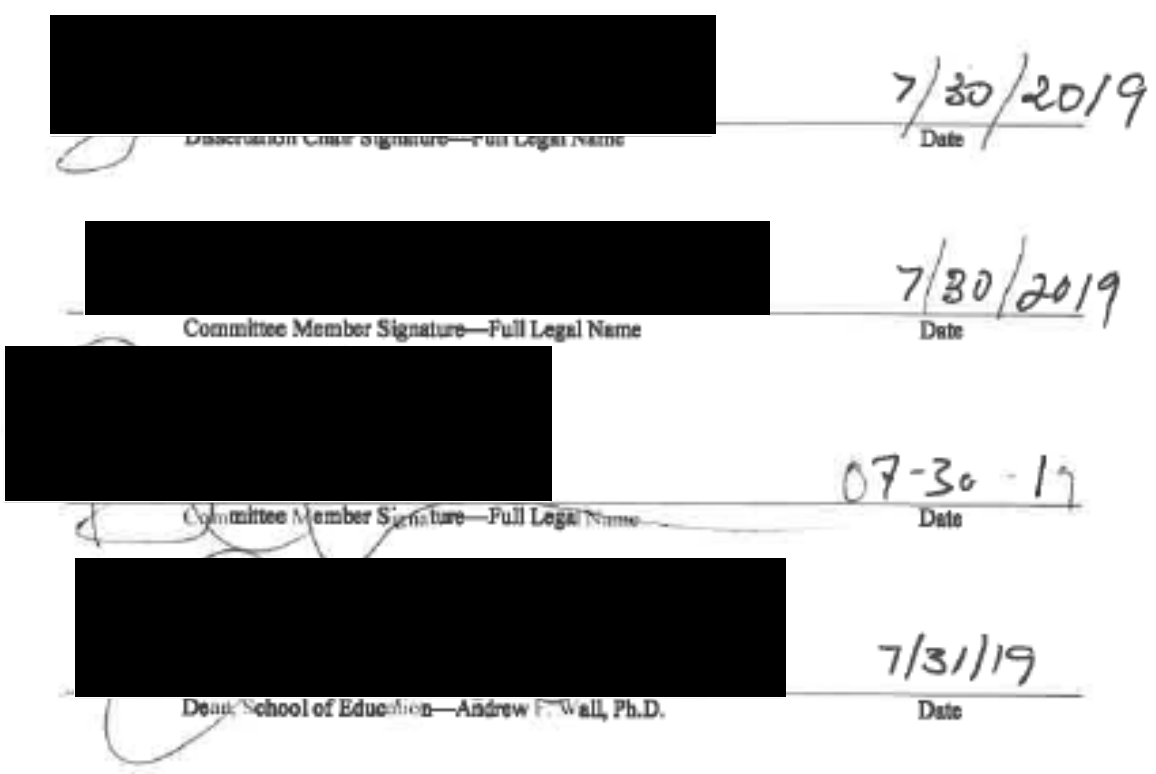

1200 East Coton Avence + PO Box 3030 - Redlands CA $92373-0999$ - Tel: 909-748-4072 - Fax: 909-335-5380 


\begin{abstract}
Examining Teachers' Paradigm and Pedagogy in Manifesting Critical Hope in Low-Income Middle School Classroom: A Narrative Inquiry.
\end{abstract}

Heidi Strikwerda

Doctor of Education, 2019

University of Redlands

Advisor: Jose W. Lalas, Ph.D.

Working in trauma-sensitive urban schools for the past 10 years steered the researcher to challenge how systemic oppression in society comfortably and continuously perpetrates the "hope gap" in low-income students, which framed the conceptual framework and the imperative work of "Critical Hope." The findings revealed the manifestations of Critical Hope in teachers' paradigm that influence their pedagogy and classroom practices. In this study, evidence of hope has been observed and identified in: (1) teachers' vocabulary expressions and written directions that connect, connote, or imply valuing and trusting optimistic aspirations to succeed, (2) teachers' written tasks and assignments that prompt critical thinking and aspirations to be caring, and (3) teachers' lesson plans that may elicit a sense of community and humanizing practices. A qualitative methodological approach using narrative inquiry was selected to seek answers to the research questions used to study, understand, and reconstruct the lived experiences of 10 teachers in the realm of practice with hope as the forum, while delving into conceptual exploration. Critical Hope paradigm-pedagogy informs teachers, administrators, and policy makers how to work effectively with children of poverty whose voices and experiences have been traditionally and historically silenced, or disregarded in the current system of education. Subsequently, other 
incidental findings revealed the danger and troubling ways common teaching practices unintentionally contribute to hopelessness. Thus, the sophistication of Critical Hope aims to inform professional development experiences for educators, administrators, and policymakers who seek to understand how to support, infuse and adopt this pedagogy that highlights hope as a transformative solution between professional development, teaching ideology and practices, and student outcomes.

Key Words: critical hope, hope gap, poverty, paradigm, engagement, social justice, equity 


\section{Dedication}

This work is dedicated to my husband, , for always believing that I could accomplish my educational and professional goals. To my children for enduring the sacrifices I have made to complete this project. Most importantly, to my Lord and savior who is my hope, counselor, and father who guides my steps each day.

"For I know the plans and thoughts that I have for you", says the Lord, "plans for peace and well-being and not of disaster, to give you a future and a hope."

Jeremiah 29:11 (The Amplified Bible) 


\section{Acknowledgements}

My sincerest gratitude is given to my mentor and chair, Dr. Jose Lalas, who inspired me to critically think about my world, to self-reflect upon my relationship to the world, and to acknowledge the responsibility we have upon those around us. This dissertation is a culmination of three years of hard work, criticality, and love. I would have never been able to complete this work on my own, nor, would I have chosen to do so if I was capable. You have opened my mind and heart to see the world in ways that have never been opened and because of you I am smarter, kinder, and stronger. "You found a light in me that I could not find." Thank you for your continued guidance and patience with me as I aspire to follow in your footsteps; to make equitable changes in education for the betterment of those who are often overlooked, overshadowed, or who have not yet found the light within them.

Salamat, ang aking magaling na propesor si Jose Lalas. Mahal kita. 


\section{Table of Contents}

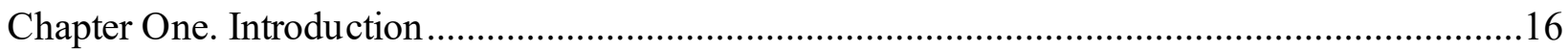

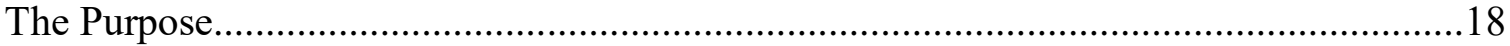

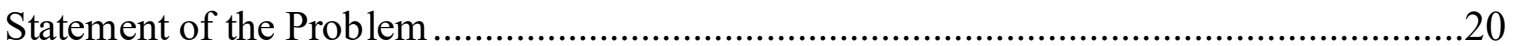

Social-Justice Leadership Perspective …......................................................20

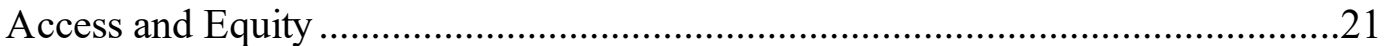

The Scourge of Poverty and Access ..........................................................22

Inequitable Inclusion through Systemic Demographics in Society .....................23

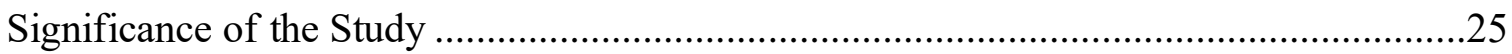

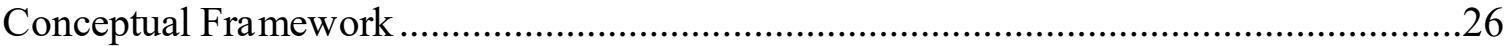

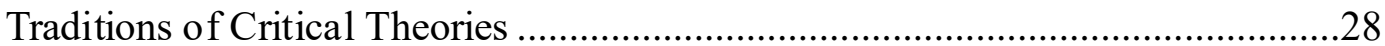

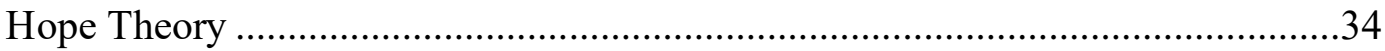

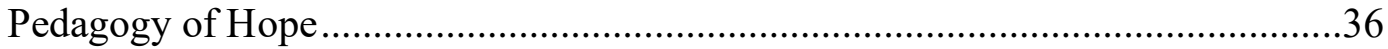

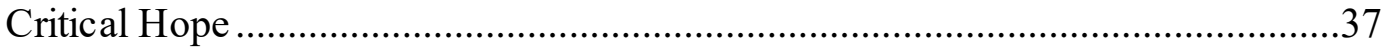

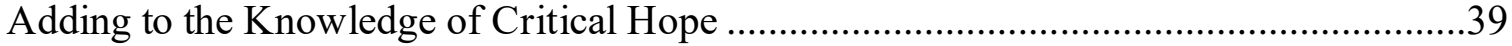

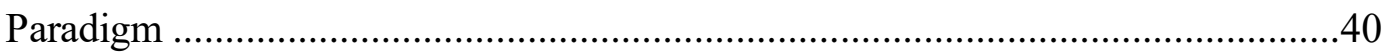

Meaning-Making and Designing .........................................................43

Individual and Institutional Paradigm............................................................44

Categories of Difference and Inequity .....................................................45

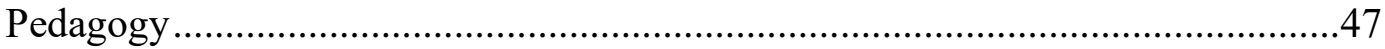

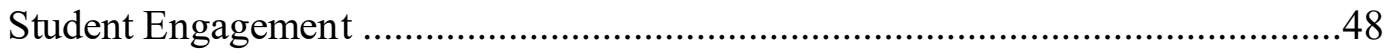

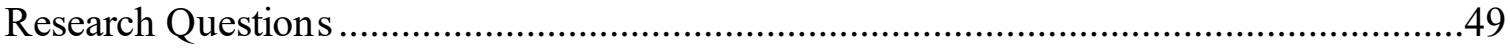

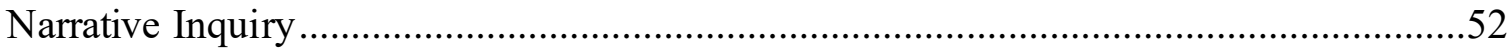




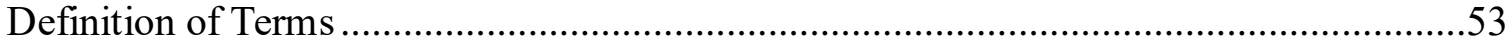

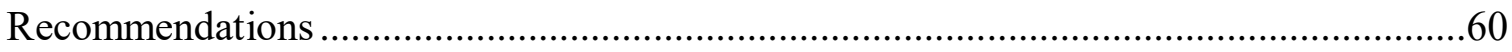

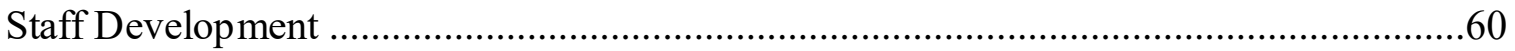

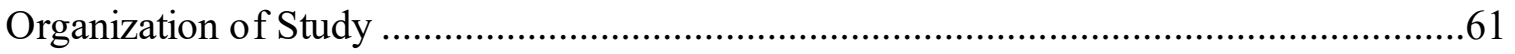

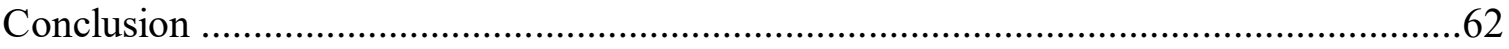

Chapter Two. Literature Review .....................................................................................64

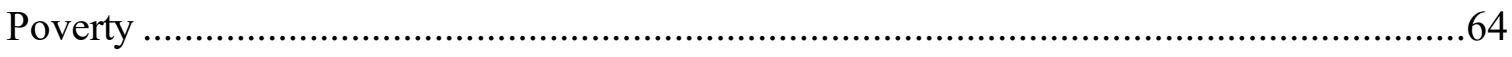

What is the Current National Poverty Level? ................................................66

Who are the People Living in Poverty? ........................................................69

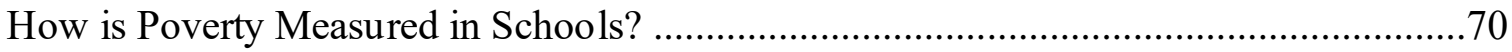

How Does the US Public Education System Receive Funding? ..................................71

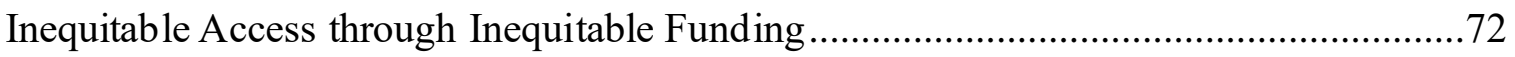

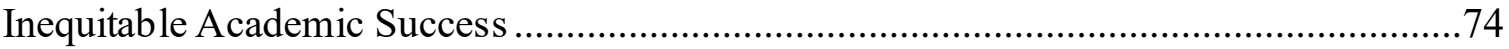

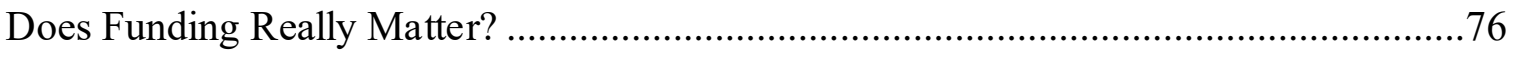

Inequitable Pedagogy: Not All Schools Are Equal (Anyon, 1980) ......................77

What Does the Surfaced Macro Data Tell Us about the US

Poverty Rate by State? .................................................................................... 78

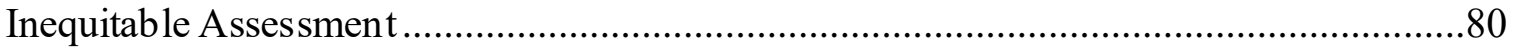

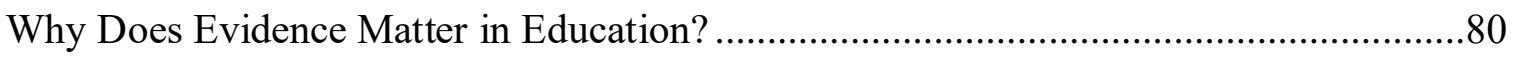

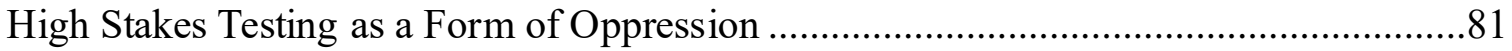

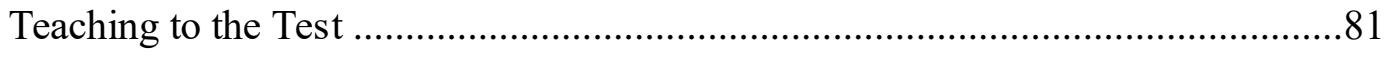

Falsification of Testing Results and Student Dropout Rates.............................82

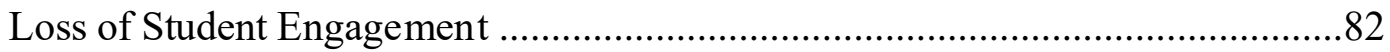


No Immediate Feedback

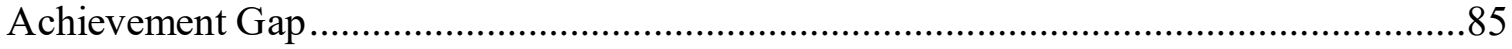

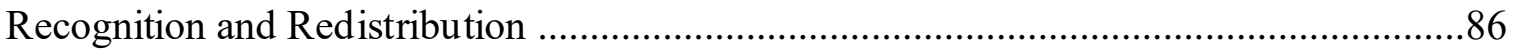

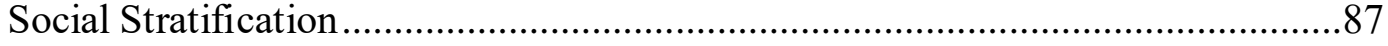

Privilege as a Form of Power and Systemic .................................................... 88

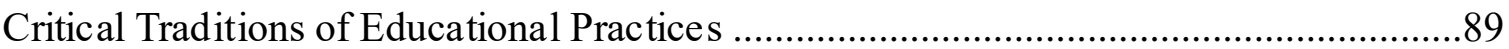

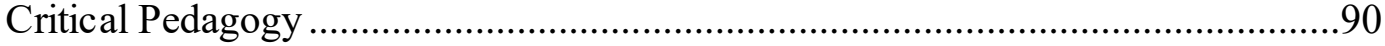

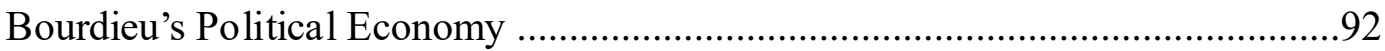

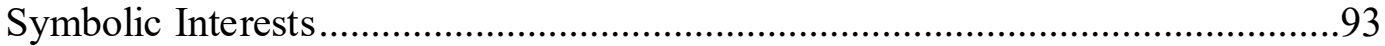

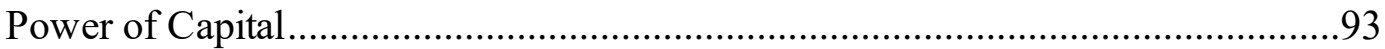

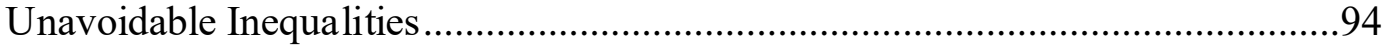

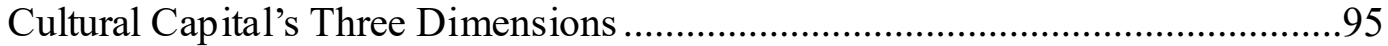

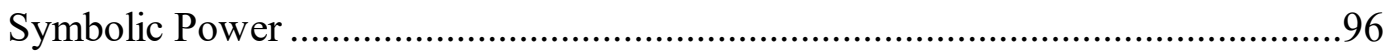

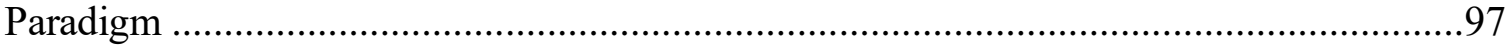

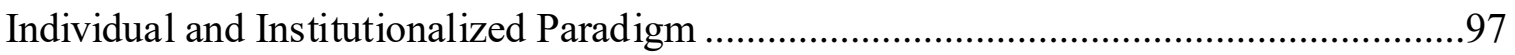

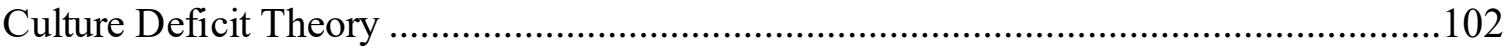

Deficit Thinking as Genetic Based ................................................................104

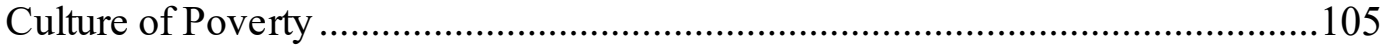

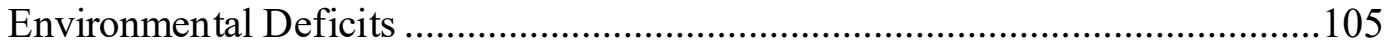

Oppressive Societal Practices and Structures ..................................................... 105

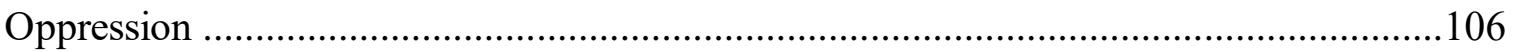

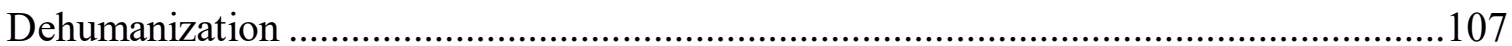




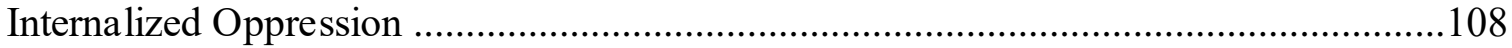

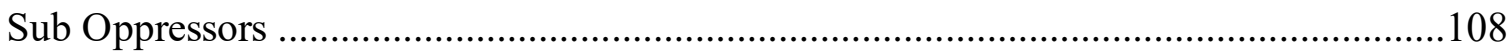

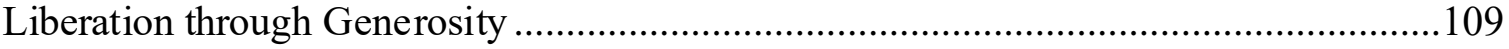

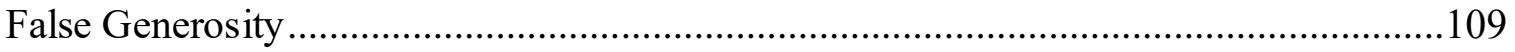

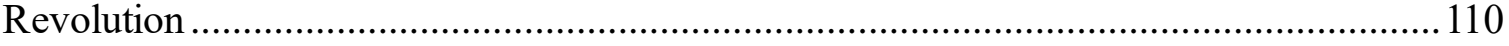

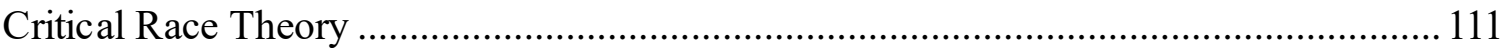

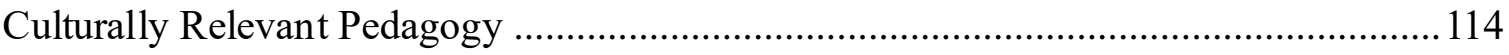

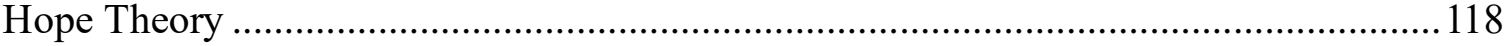

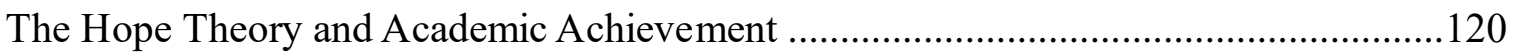

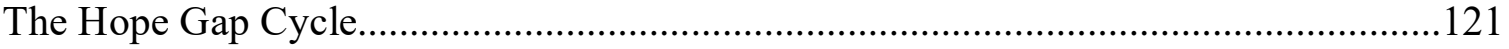

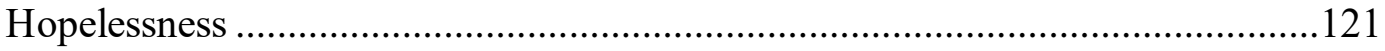

Hope, Motivation, and Student Engagement ................................................123

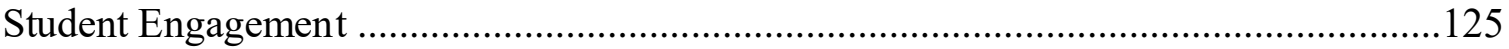

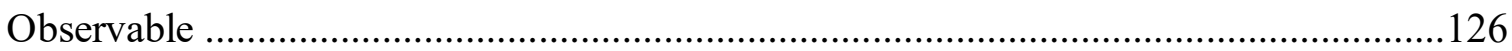

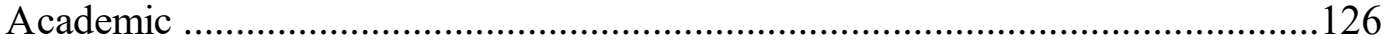

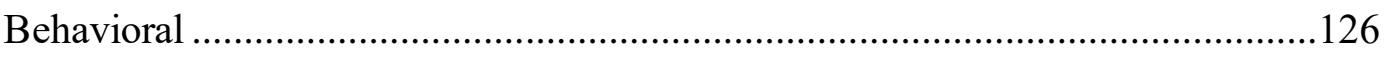

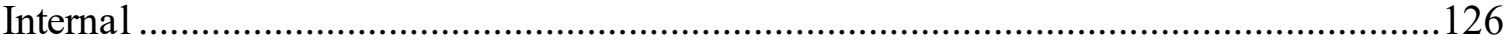

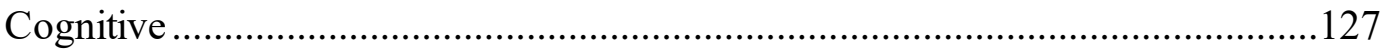

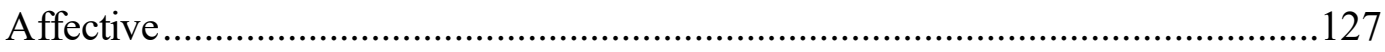

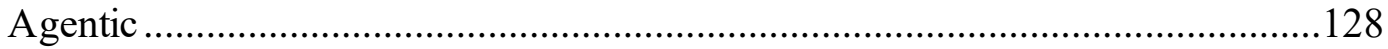

How Do We Break the Hope Gap Cycle? ......................................................129

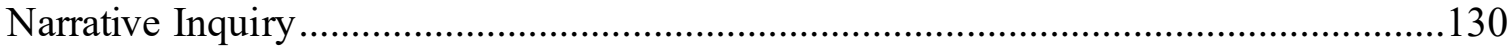

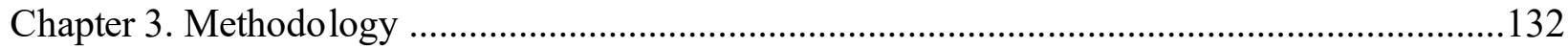


Introduction

Research Design

Narrative Inquiry

Data Collection Procedure

Setting

Sampling

Participants.

Positionality

Gate Keeper.

Protection of Participants

Potential Risks of the Study

Instrumentation

Data Collection Analys is.

Summary

Research Questions

Purpose.....

Data Analysis

Process

Findings

Themes and Categories .

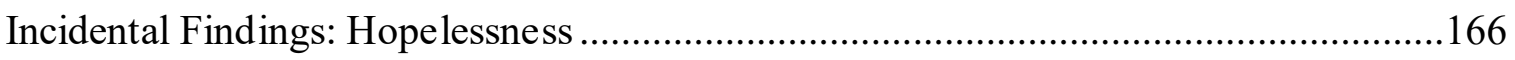

Incidental Findings: Unintentional Hopelessness 


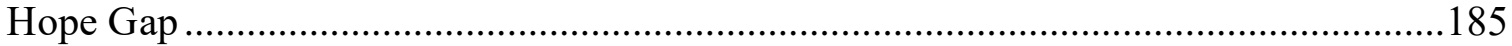

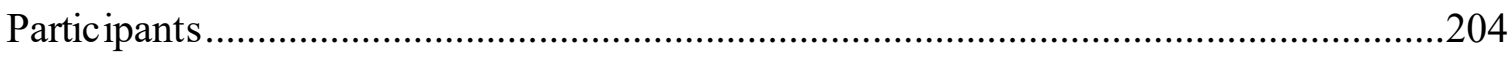

Shift in Conceptual Framework ..................................................................................208

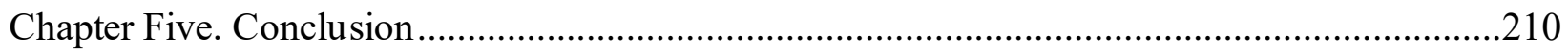

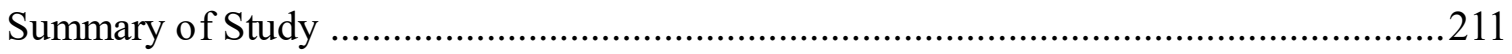

Research Questions Discussion .......................................................................212

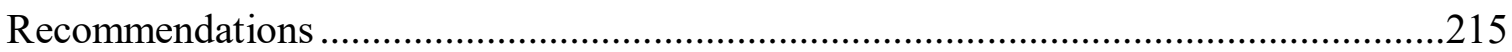

From Freire (1994) to Current Formulations of Critical Hope ........................................218

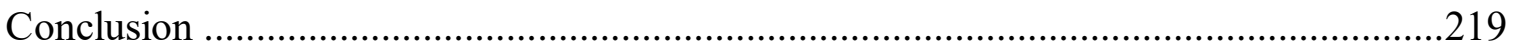

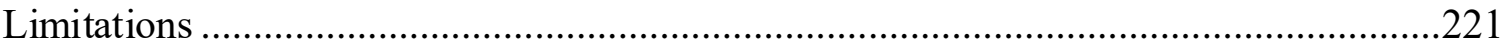

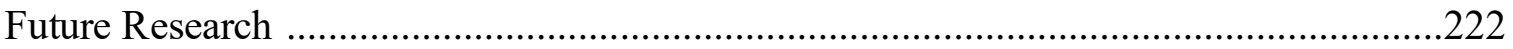

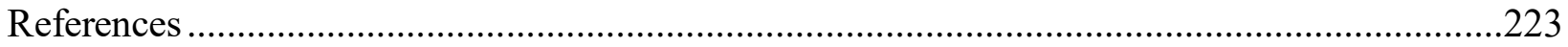

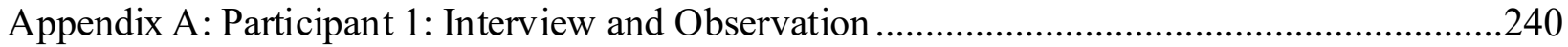

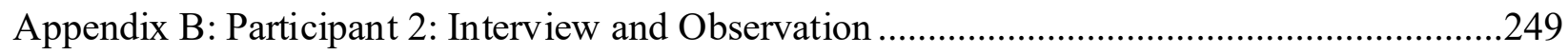

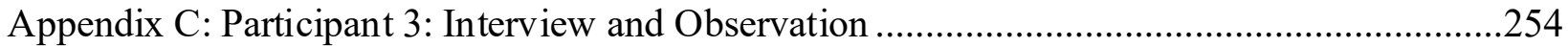

Appendix D: Participant 4: Interview and Observation...........................................................260

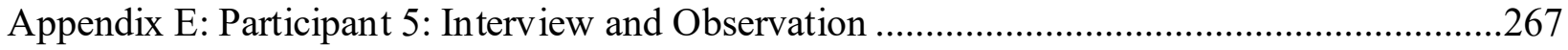

Appendix F: Participant 6: Interview and Observation .........................................................273

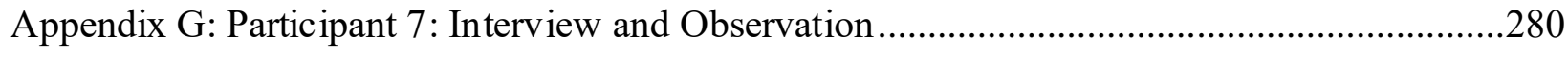

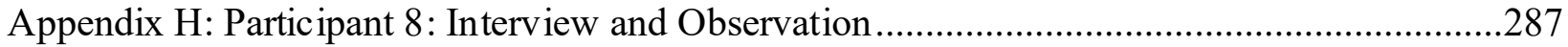

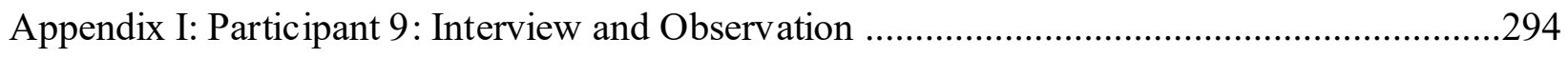

Appendix J: Participant 10: Interview and Observation .........................................................298 
EXAMINING TEACHERS' PARADIGM AND PEDAGOGY IN MANIFESTING CRITICAL

HOPE IN LOW-INCOME MIDDLE SCHOOL

\section{List of Tables}

Table 1 Highest Poverty Rate verses Lowest Academic Achievement ..................................73

Table 2 Critical Traditions of Educational Practices (CTEP) .............................................. 112

Table 3 From Freire (1994), to Duncan Andrade (2008), to Current Formulations of Critical Hope Based Upon Research Strikwerda (2019) 


\section{List of Figures}

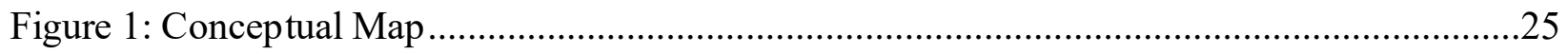

Figure 2: Official and Supplemental Poverty Measures ..................................................61

Figure 3: Poverty Rate Over 50 Years ..........................................................................62

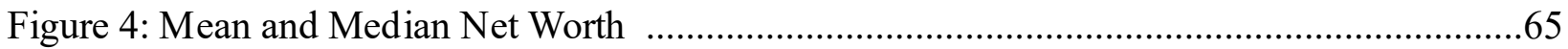

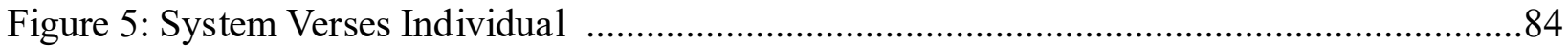

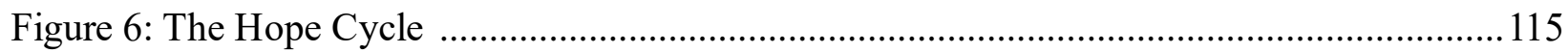

Figure 7: Cycles of the Hope and the Hopeless ............................................................... 118

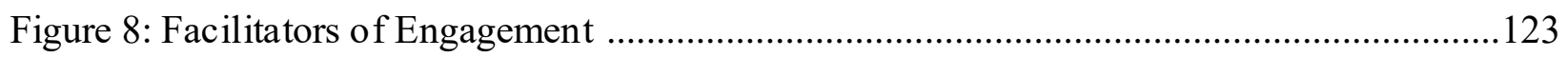

Figure 9: Raising Levels of Hope Produces Change in Motivation and Engagement ...............124

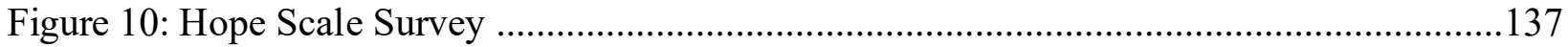

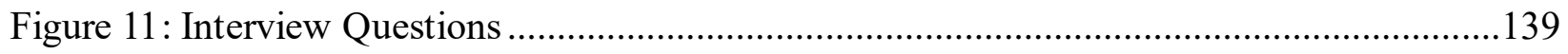

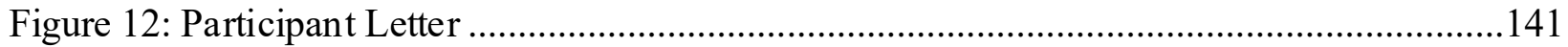

Figure 13: Manifestations of Critical Hope in Teachers' Paradigm and Pedagogy

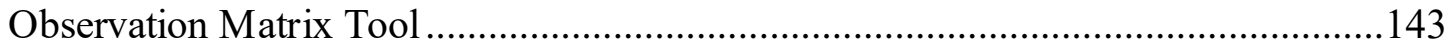

Figure 14: Manifestations of Hope in Teachers' Paradigm and Pedagogy ..............................153

Figure 15: Words Related to Hope as Reflected by Teachers' Voices .....................................156

Figure 16: Goal Oriented, Willingness to Act, and Perseverance .........................................157

Figure 17: Building Trust, High Expectations, and Caring Relationships ..............................158

Figure 18: Critical Reflection, Advocacy, Citizenry, and Empowerment...............................159

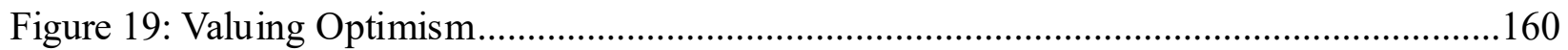

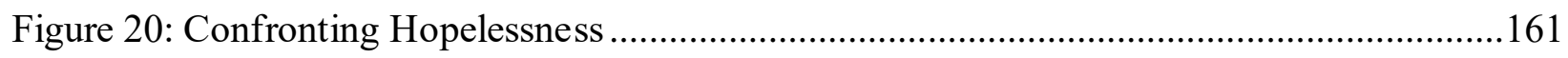

Figure 21: Personal Commitment to Ethical Responsibility and Accountability ......................162

Figure 22: Humanizing Self-Identity and Sen se of Community ..........................................163 
EXAMINING TEACHERS' PARADIGM AND PEDAGOGY IN MANIFESTING CRITICAL HOPE IN LOW-INCOME MIDDLE SCHOOL

Figure 23: Consciousness of Economic, Social, Cultural, and Historical Life Barriers 164

Figure 24: Inspiring Motivation and Engagement

Figure 25: Manifestations of Hopelessness in Teachers' Paradigm and Pedagogy 166

Figure 26: Not Goal Oriented, Depleted Willpower, and Deficiency in Coping .....

Figure 27: Mistrusts, Low Expectations, and Deficiency in Social Skills

Figure 28: Uncritical, Feels Controlled by Outside Forces, Lacks Power, and Citizenship Skills

Figure 29: Learned Pessimism 172

Figure 30: Ignoring Hopelessness

Figure 31: Professional Deflection and Lacks Ethical Accountability

Figure 32: Dehumanizing Self-identity and Sense of Community 175

Figure 33: Unconsciousness of Economic, Social, Cultural, and Historical Life Barriers 176

Figure 34: Apathetic, Unmotivated, and Disengaged 177

Figure 35: Incidental Findings Unintentional Hopelessness

Figure 36: Unintentional Hopelessness Symbolic Violence, Misrecognition, and Unstable Environments 180

Figure 37: Unintentional Hopelessness Self Deception

Figure 38: Unintentional Hopelessness Self Depreciation and Narration Sickness 185

Figure 39: Theme 1 Goal Oriented, Willingness to Act and Perseverance the Hope Gap and Not Goal Oriented, Depleted Willpower, and Deficiency in Coping Skills

Figure 40: Theme 2 Building Trust, High Expectations, and Caring relationships, the Hope Gap, and Mistrust, Low Expectations, and Deficiency in Social Skills 188

Figure 41: Theme 3: Valuing Optimism, the Hope Gap, and Learned Pessimism 190

Figure 42: Theme 4: Confronting Hopelessness, the Hope Gap, and Ignoring Hopelessness 
Figure 43: Theme 5 Personal Commitment to Ethical Responsibility and Accountability, the Hope Gap, and Professional Deflection and Lacks Ethical Accountability

Figure 44: Theme 6 Humanizing Self Identity and Sense of Community, the Hope Gap, and Dehumanizing, Low-Self Esteem, and Disconnected

Figure 45: Theme 7 Consciousness of Economic, Social, Cultural, and Historical Life Barriers, the Hope Gap, and Unconscious of Economic, Social, Cultural, and Historical Life Barriers

Figure 46: Theme 8 Critical Reflection, Advocacy, Citizenry, and Empowerment, the Hope Gap, and Uncritical, Feels Controlled by Outside Forces, Lacks Power, and Citizen ship Skills

Figure 47: Theme 9 Inspiring Motivation and Engagement, the Hope Gap, and Apathetic, Unmotivated, and Disengagement

Figure 48: Two Positions that Emerged from Teachers' Voices: Hopeful and the Hopeless as a Visual Display of the Hope Gap

Figure 49: Site Demographics 205

Figure 50: Participants 206

Figure 51: New Conceptual Framework 210

Figure 52: Invisible Factors that May Relate to the Continual and Existing Achievement Gap between Affluent Students and Low-Income Students

Figure 53: Critical Hope and Its Influence on Students Engagement. .222 
EXAMINING TEACHERS' PARADIGM AND PEDAGOGY IN MANIFESTING CRITICAL HOPE IN LOW-INCOME MIDDLE SCHOOL

\title{
Chapter One. Introduction
}

\author{
Examining Teachers' Paradigm and Pedagogy in Manifesting Critical Hope \\ in Low-Income Middle School Classroom: A Narrative Inquiry
}

I do not believe most educators enter their classrooms prepared to make transformative, equitable solutions that meet the needs of all their students when they need them (Lalas, Charest, Strikwerda, \& Ordaz, 2019). The current K-12 educational system comfortably functions year after year unconscious of how normal practices unintentionally contribute to and create hopelessness in both educators and the students of poverty who are also those with the greatest need. Our inability to challenge the common sense (Kumashiro, 2015) of pervasive paradigms and pedagogical practices that provide the foundations for permitting and continuing the status quo unjustly, inequitably, and historically, reinforce the miseducation children of poverty receive generation after generation, diminishing their hope, and preventing them from meeting their full potential (Blankstein, Noguera, \& Kelly, 2016). As it is not easy to provide the equitable pedagogical practices needed to combat the systemic oppression students of poverty face, it is even harder for those who have experienced success in the traditional educational system to recognize how it continually leads to disenfranchisement for students of poverty who are often racial and ethnic minorities and who are not privy to the privilege they themselves receive.

According to Berliner (2005),

What is it like for children to grow up here? What do they think the world has done to them? Do they believe they are being shunned or hidden by society? If so, do they think that they deserve this? What is it that enables some of them to pray? And when they pray, what do they say to God? (p. 54) 
EXAMINING TEACHERS' PARADIGM AND PEDAGOGY IN MANIFESTING CRITICAL HOPE IN LOW-INCOME MIDDLE SCHOOL

Freire (1994) discusses how hope cannot be ignored, as hope is an ontological need, important for one's existence, and a necessary attribute for one to engage in the struggle and transformation of the world. One cannot hope and not act, as to simply hope, is to, "hope in vain" (Freire, 1994, p. 3). Hope in itself is not enough because without action, hope turns into hopelessness and despair. Upon analysis of the social, historical, and economic situations for children of poverty, the progressive educator takes care in identifying the purpose for engaging in an education of hope as Freire (1994) states, "we need Critical Hope the way a fish needs unpolluted water" (p. 2). Similarly, the critical pedagogue asks questions about the construction of knowledge and power relationships seeking to bring equitable transformations in the world (Brookfield, 2005). Other related questions may include: What social, cultural, and historical constructs and power relations exist in education that intentionally and unintentionally perpetrate social inequalities and injustices for children of poverty? How do paradigms and pedagogy manifest hope for children of poverty? How do paradigms and pedagogical practices diminish their quest to be successful? How does one formulate pedagogy of Critical Hope? Historically, how have we addressed the societal failure of pervasive poverty in America, and are these transformative solutions?

During President Lyndon B. Johnson's first state of the union address on January 8, 1964, he declared an "unconditional war on poverty" as he believed that high levels of poverty in America are a national disgrace representing a deeper societal failure (Cooley, 2018). President Johnson famously said, "Our aim is not only to relieve the system of poverty, but to cure it and above all, to prevent it" (Rector \& Sheffield, 2011, p. 6). After the Civil Rights Act was signed into law on July, 2, 1964, inequitable amounts of poverty in America became the main legislative focus, as civil rights activists pressured government on economic racial disparities (McKee, 
2010). Even though President Lyndon B Johnson's intentions were honorable by seeking a cure for poverty, and signified a cry for justice within society based on the notion of equality for humanity, he failed to see the systemic causes that continually reinforce the poverty cycle in America. The "Great Society" was an ineffective, affirmative solution placing responsibility solely on the persons of poverty, instead of addressing the combined root causes as a whole, as Johnson's ideology for combating the war on poverty focused on equipping the poor through government assistance, with the expectations that they would pull themselves up out of poverty (McKee, 2014). Therefore, Johnson's ideology failed to be a transformative solution, as it did not attack poverty and equality at the root. In order for equality for humanity to exist, equality of systemic conditions is also needed (Schneiderman, 2017).

More than 50 years later, America continues to experience a degree of inequality along social class, racial, and ethnic lines in society which remain and are reinforced by the belief that anyone can be pulled out of poverty by his or her bootstraps (Howard, 2010). In fact, economic inequality has become demonstrably worse in subsequent years. According to Flint (2018), "Of all the ways in which inequality hinders American progress, gaps in educational achievement are perhaps the most pernicious" (p. 24). In reality, children of poverty are facing educational inequities, which continue to translate into lower academic achievement as measured by standardized test scores (Berliner, 2005; Ullucci \& Howard, 2015). Thus, if we want to solve the academic problems we perceive in low-income schools, we need to also address the poverty issue.

\section{The Purpose}

The purpose of this dissertation was to examine, employ, and formulate the notion of "Critical Hope" as a transformative solution for children of poverty. For the purpose of this 
EXAMINING TEACHERS' PARADIGM AND PEDAGOGY IN MANIFESTING CRITICAL HOPE IN LOW-INCOME MIDDLE SCHOOL

dissertation, "Critical Hope" is defined as an optimistic way of viewing and acting on the world from a critically historical conscious, socially and culturally situated perspective with the intention to care, endure, and sustain effort in the struggle against current forms of oppressive conditions with the belief that change is inevitable and inspires community, advocacy, liberation, and justice. "Critical Hope" is a multidisciplinary approach including sociology, history, education/pedagogy, economics, philosophy, psychology, and social justice where one analyzes all the contributing factors that may influence one's visualized trajectory and belief in their capacity to succeed in the future. Specifically, "Critical Hope" will be used to analyze and address educational inequities children of poverty face within the societal structures, and to suggest ways that educational leaders can become "agents of hope" for their students, which may translate to equitable access and academic success. First, in order to understand the educational challenges that children of poverty face in education and employ remedies to these inequities, a brief discussion about the effects of inequality and injustice on students in schools serving lowincome communities, which I will refer to as the "plight of children of poverty" will be explored through a socially-just perspective. Next, the traditions of critical theories of educational practice and the hope theory will be discussed, integrated, and formulated as a perspective or set of lenses that projects the notion of "Critical Hope." This will lead to the discussion of the need to employ "Critical Hope" for children of poverty as a remedy to increase educational access and success. Finally, recommendations have been made which may influence local school districts' professionals learning about the importance of highlighting Critical Hope as a paradigm and pedagogical shift to inform teachers, administrators, and policy makers how to work effectively with children of poverty whose voices and experiences have been traditionally and historically silenced, or disregarded in the current system of education. 
EXAMINING TEACHERS' PARADIGM AND PEDAGOGY IN MANIFESTING CRITICAL HOPE IN LOW-INCOME MIDDLE SCHOOL

\section{Statement of the Problem}

In this section, this research specifically addresses the plight of children of poverty specifically pertaining to inequitable educational access leading to inequitable academic outcomes for students in low-income areas. In a recent article, The Price of Equality: the Achievement Gap and the High Cost to America's Future (Flint, 2018), the author discusses how the present social and economic inequality in America is a result of inequitable education. Flint (2018) reported that low-income students are about 31 points behind their peers who do not belong to low-income families in both Mathematics and Language Arts, and most of the lowachieving students who belong to low-income families are Hispanic and African American (Flint, 2018). According to Flint (2018), children of poverty are not performing as well as their affluent counterparts because they do not have equal access to quality education. Thus, children of poverty suffer from educational inequity and require attention from a more critically and socially- just perspective

\section{Social-Justice Leadership Perspective}

From a socially-just perspective, Theoharis (2007) employed social-justice leadership (SJL) which is defined as having leaders who embrace inclusion as a model for education in order to create a more equitable world for historically and marginalized students who face inequitable educational conditions. In this definition, inclusion pertains to all students who have been traditionally segregated and historically underserved. In this way, social-justice leadership recognizes inequity and advocates on behalf of students who are poorly served. Leaders who embrace a social-justice perspective seek to diminish hierarchies of power as well as structures and systems that privilege some students and oppress others (Jean-Marie, Normore, \& Brooks, 2009). Thus, a discussion is needed on the plight of children of poverty through a socially-just 
EXAMINING TEACHERS' PARADIGM AND PEDAGOGY IN MANIFESTING CRITICAL HOPE IN LOW-INCOME MIDDLE SCHOOL

perspective in order to create changes that will address these inequities related to educational access and academic success.

\section{Access and Equity}

According to Brown v. Board of Education (1954),

In these days it is doubtful that any child may reasonably be expected to succeed in life if he is denied the opportunity of education. Such an opportunity, where the state has undertaken to provide it, is a right, which must be made available to all on equal terms .... (Flint, 2018, p. 22)

Sixty-five years have passed since this monumental statement was made regarding separate but equal access to education and segregation. However, the right to education in terms of equality continues to remain elusive (Flint, 2018). Having equal access to quality education is a matter of social justice. Social justice is concerned with respect for all human rights, equality, and fairness. It examined relationships concerning populations who are systematically not given the same opportunities, resources, experiences, and advocates for change within injustices (CochranSmith et al., 2016). Advocates for social justice differentiated between the concepts of equality and equity.

According to a key text in social stratification, the central thesis of Kingsley David with Wilbert Moore (1945), Some Principal of Stratification,

"Equality of conditions is possible, but inequality is inevitable, because to fill their most important positions with the most capable individuals, all societies all have to reward leaders differentially, with high wealth and high prestige” (Schneiderman, 2017, p. 5).

Social stratification refers to the concept that social groups are relationally positioned and ranked into hierarchy of unequal value and is used to justify unequal resources among groups 
EXAMINING TEACHERS' PARADIGM AND PEDAGOGY IN MANIFESTING CRITICAL HOPE IN LOW-INCOME MIDDLE SCHOOL

(Sensoy \& DiAngelo, 2012). Equality refers to sameness: same opportunity, the same rights, and the same treatment (Cochran-Smith et al., 2016). Similarly, inequality refers to difference in opportunities, conditions, provisions, and rights. Equity is theorized as the relevant and appropriate instructional, curricular, and programmatic attempt to meet the needs of all students when they need them (Blankstein, Noguera, \& Kelly, 2016; Lalas, Charest, Strikwerda, \& Ordaz, 2019). Equity goes deeper than equality and is concerned with justice or injustices within marginalized groups of people within society (Cochran-Smith et al., 2016). Social Justice in education is concerned with equal participation of all groups in society and is focused on meeting their individual needs. For the purpose of this dissertation, social justice in education refers to the ability for all students to have equal access to quality education in preparation of becoming change agents in society (Bell, L., 2007).

\section{The Scourge of Poverty and Access}

The reality is that poverty creates daily obstacles that influence equality of life conditions affecting the development of social, emotional, and academic success (Berliner, 2005). Children of poverty experience disproportionate levels of high crime, gang violence, drug influence, death, and health issues (Ullucci \& Howard, 2015) which often lead to develop what Rawlinson (2011) described as a poverty mindset. A poverty mindset leads to feelings of powerlessness, lack of control over their lives, and internalized failure as a lack of ability, rather than skill. "Many students in poverty have spotty-to-poor academic records that can often be linked to the poverty mindset that strips students of ambition and enthusiasm and makes them indifferent" (Rawlinson, 2011, p. xiii).

The result of this poverty mindset is what Rawlinson (2011) defined as a "scourge of poverty," resulting in low academic achievement scores, high behavioral problems, and a 
continual message that plays in students minds that they are unworthy. These messages of unworthiness are internally accepted, as they play over and over in their heads, diminishing desire to pursue goals, or to try new things. The challenge lies for both the student and the educator as the student internally accepts messages of failure, which is demonstrated through outward expressions of frustration and resentment due to their discouragement. Students then begin to feel a sense of hopelessness, are disengaged from school, and become depressed resulting in Educators who begin to label students as problems, apathetic, and unmotivated. Thus, the vicious cycle continues as a lack of belief in abilities is manifested in both the educator and the student leading to unwanted student behaviors and lack of student achievement.

The reality is that the poverty mindset is one of the "most difficult and pervasive challenges to overcome" (Rawlinson, 2011, p. xv). Teachers of children of poverty are often lacking in training and experience on how to connect, teach, and manage students who experience the scourge of poverty (Ullucci \& Howard, 2015). Inequitably, the areas of most academic need are also the most difficult to staff classrooms with well-trained, experienced teachers with the skills and knowledge needed to generate successful learners in low-income student populations who continually experience the scourge of poverty (Ullucci \& Howard, 2015). As a result, children of poverty do not have equitable access to a quality education, which will meet their behavioral, social, and academic needs. This current study attempted to identify the role of hope in recognizing the needs of children of poverty and paying attention to the educational and social needs resulting from scourge of poverty in order to mediate the inequities and influence the level of hope one has for their future.

\section{Inequitable Inclusion through Systemic Demographics in Society}

Inequitable inclusion through demographics is a factor for children of poverty as 
EXAMINING TEACHERS' PARADIGM AND PEDAGOGY IN MANIFESTING CRITICAL HOPE IN LOW-INCOME MIDDLE SCHOOL

segregation continues to exist in our neighborhoods by social class and race (Berliner, 2005).

Data from the U.S. Census Bureau (2018) revealed that the highest level of poverty continues to reside with Blacks at $22 \%$, followed by Hispanics at $19.4 \%$, and lastly with Whites at $8.8 \%$ (Peter G. Peterson Foundation, 2018). This demonstrates that students in poverty are primarily minorities composed of diverse people of color. In fact, demographic regions containing high levels of poverty in the United States are often homogenous and less diverse than before the Brown v. Board of Education (1954) decision (Hakuta, 2017). As a result, data revealed that race and poverty perpetually intersect (Ullucci \& Howard, 2015). It is imperative that social-justice leaders must critically examine the relationship between poverty and children of color as America continues to experience levels of economic inequality (Flint, 2018).

Current literature on the plight of children of poverty in American public schools revealed the presence of inequities in the following areas: inequitable inclusion through systemic demographics (Berliner, 2005; Education Trust-West, 2017; Howard, 2010), inequitable pedagogy and expectations between affluent schools and low-income schools (Anyon, 1980; Berliner, 2005; Chen \& Hahnel, 2017; Duncan-Andrade, 2009), inequitable funding between affluent areas and low-income schools (Chen \& Hahnel, 2017; Flint, 2018), inequitable access to properly trained teachers (Berliner, 2005; Lalas, 2007; Lushei \& Jeong, 2018), inequitable professional learning opportunities (Berliner, 2005; Chen \& Hahnel, 2017; Lalas, 2007; Schmoker, 2012), inequitable access to appropriate assessment (Darling-Hammond, AmreinBeardsley, Haertel, \& Rothstein, 2012; Flint, 2018), inequitable access to resources and adequate facilities (Chen \& Hahnel, 2017; Flint, 2018; Ladson-Billings \&Tate, 1995), inequitable access to rigorous, college preparatory coursework in all content areas (Chen \& Hahnel, 2017), access to counselors and librarians (Chen \& Hahnel, 2017), and intense segregation in schools 
EXAMINING TEACHERS' PARADIGM AND PEDAGOGY IN MANIFESTING CRITICAL HOPE IN LOW-INCOME MIDDLE SCHOOL

(Education Trust-West, 2017; Flint, 2018), which are all factors leading to inequitable academic success (Education Trust-West, 2017; Flint, 2018).

\section{Significance of the Study}

The significance of this qualitative study was to examine low-income middle school teachers' perceptions of hope that manifested in their paradigm and pedagogy and how this determination may influence the inclusion of hope as an offering in professional-learning opportunities for teachers and administrators in local school districts. Conceptually, this study revealed through teachers' voices how their paradigm and pedagogy informs their desire for their students to be hopeful in their quest for success. Theoretically, the research objective necessitated the use of critical theories in education such as Freire's education for critical consciousness (Freire, 1974), pedagogy of the hope (Freire, 1994), and pedagogy of heart (Freire, 1997), bell hooks' (2003) pedagogy of hope, Giroux's (1997) pedagogy and politics of hope and other related critical theories (Anyon, 1980; Bourdieu, 1986; Brookfield, 2005; Dewey, 1938; Duncan-Andrade, 2009; Freire, 1970; Giroux, 2011; Murphy \& Fleming, 2010; LadsonBillings \& Tate, 1995; Noddings, 2017; Saltman, 2018) and the Hope Theory (Edwards, Rand, Lopez, \& Snyder, 2007) as the conceptual framework in order to formulate a pedagogy of "Critical Hope." The practical and educational value of the proposed project was to provide an opportunity to identify classroom practices that reflect hope as they emerge in the authentic voices of teachers.

Voices of teachers sympathetic to low-income students are defined as those who are not valuable and often overshadowed by the voices of teachers from the dominant culture (Sensoy \& DiAngelo, 2012). The regular classroom is a space of neutral and normal schooling that is socially constructed to accommodate the ways that children of the dominant culture learn 
EXAMINING TEACHERS' PARADIGM AND PEDAGOGY IN MANIFESTING CRITICAL HOPE IN LOW-INCOME MIDDLE SCHOOL

(Sensoy \& DiAngelo, 2012). This current educational system creates and maintains segregation and privilege for the dominant group over the marginalized group, which reinforces the educational experiences of low-income students related to teacher paradigm and pedagogy (Ladson-Billings \& Tate, 1995; Sensoy \& DiAngelo, 2012). According to Ladson-Billings and Tate (1995), this system has reproduced the status quo in society related to race and social class.

The dissertation aims to provide stories for teachers, administrators, and policy makers to listen to and understand the perspectives of teachers of children of poverty whose voices and experiences related to hope have been silenced or disregarded in the current system of education. Upon analysis of these stories, the expectation is that themes will emerge pertaining to classroom paradigm and pedagogy and their alignment to the concepts on hope synthesized from the aforementioned critical theories. Existing research on hope demonstrated that students who develop high levels of hope, engage in school, and persevere through tasks better lead toward academic achievement, and students who have lower levels of hope are more likely to disengage from school and have negative school outcomes (Edwards et al., 2007). Thus, the purpose of this dissertation is not to make the connection between hope and academic achievement as that has already be identified through existing empirical research, but rather the purpose of this dissertation is to formulate a conceptual framework on Critical Hope and support it empirically with data that examines the manifestations of Critical Hope that emerged in teachers' paradigm and pedagogy of low-income middle school classrooms.

\section{Conceptual Framework}

The current study was guided by the formulation of the notion of "Critical Hope" and its impact on student engagement. First, a discussion of traditions of critical theories of educational practice will take place. Next, a discussion of the hope theory will occur leading to the creation 
of the "Critical Hope" conceptual framework. Then a discussion on paradigm and pedagogy will take place. Finally, a discussion on student engagement and engagement types occur. Figure 1 shows this dissertation's conceptual framework.

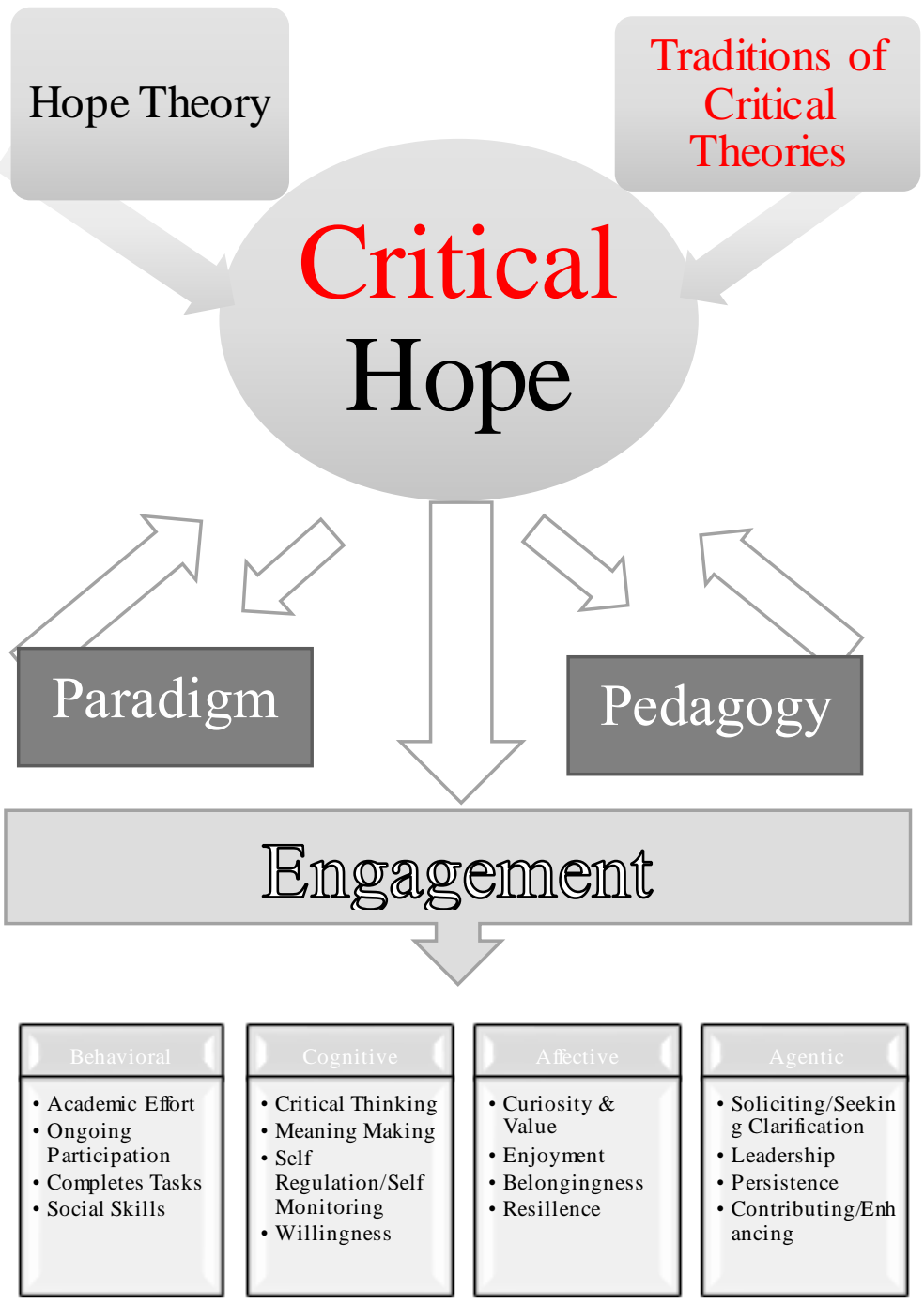

Figure 1: Conceptual Map 
EXAMINING TEACHERS' PARADIGM AND PEDAGOGY IN MANIFESTING CRITICAL HOPE IN LOW-INCOME MIDDLE SCHOOL

\section{Traditions of Critical Theories}

Figure 1 is a visual representation of the conceptual framework depicting the formulation of "Critical Hope" which is a combination of the traditions of critical theories of educational practice and the hope theory. First, the elements of the traditions of critical theory in education that form "Critical Hope" are critical optimism, ethics, criticality, social and historical consciousness, sense of community, caring relationships, knowledge of social and cultural capital, democratic advocacy, and humanizing practices (Anyon, 1980; Bourdieu, 1986; Brookfield, 2005; Dewey, 1938; Duncan-Andrade, 2009; Freire, 1997, 1994, 1974, 1970; Giroux, 2011, 1997; Murphy \& Fleming, 2010; hooks, 2003; Ladson-Billings \& Tate, 1995; Noddings, 2017; Saltman, 2018). These elements attempt to empower students as critical citizens informed by the knowledge of politics, societal structures, powers of domination, subject positions, self-reflection, responsibility, and advocacy to change the world in which they live (Giroux, 2011). As a result, these elements are considered humanizing practices of freedom (Freire, 1970) that provide freedom, criticality, praxis, and autonomy the teachers that empower students as they develop a critical consciousness of the world, and their place in relation to the world (Giroux, 2011).

On critical optimism, Freire (1997) discussed that transformation of the world begins with reading the world and preserving the hope that change is possible. "A new reading of my world requires a new language- that of possibility, open to hope" (Freire, 1997, p. 37). This language of hope includes a critical consciousness of the injustices in the world expressed with words of goodness and peace by those who are engaged in the struggle, and are in search of permanent possibilities that may alleviate the inequities of the oppressed (Freire, 1997). "Critical optimism requires a strong sense of social responsibility and of engagement in the task 
of transforming society, it cannot mean simply letting things run on" (Freire, 1974, p. 11). Thus, it is impossible to begin the search without hope, as critical optimism is where the resistance to oppression begins with ideals of possibility in the transformation and restoration of the present world.

On ethics Freire (1997) stated that the discourse of those involved in the struggle must be "hopeful, critically optimistic, and drenched in ethics" (p. 43). The ethics he referred to is related to the improvement of democracy by a process of unification against all social injustices. "On this level, the struggle for hope means the denunciation, in no uncertain terms, of all abuses, schemes, and omissions. As we denounce them, "we awaken in others and ourselves the need, and also, the taste for hope" (Freire, 1997, p. 59). For Freire (1997), ethics includes the uniting of all groups who face injustices for one common good, so they will see themselves as connected to their community, instead of each group competing and fighting their individualized battles in isolation. Freire (1997) also asserted that if "each group is fighting its own battles...we tend to divide forces, fighting among and against ourselves, instead of fighting the common enemy" (p. 44). Freire (1997) believed that more is needed to remove inequities, a "unity within diversity" by forging a greater fight, a greater dream, a greater hope that ethically unites what each party aspires for. Thus, ethics involves a unification of all those who have been marginalized in society by the dominant culture, to communally fight against social injustices as a whole.

On criticality there is a responsibility placed upon the educator to reclaim schooling as an emancipatory process involving what Freire (1997) described as the "possibility of knowing" (p. 50). This includes expanding the possibilities of critical thinking through a reading of the world and the word, dialogue, problem-posing education, and fostering curiosity. According to 
Giroux (2011), "critical thinking represents the ability to step beyond commonsense assumptions and to be able to evaluate them in terms of their genesis, development, and purpose" (p. 40). In this way, criticality is a political act where "human beings must emerge from their own submersion and acquire the ability to intervene in reality as it is unveiled (Giroux, 2011, p. 41). Criticality creates an awareness of the world through a linking of historical consciousness of how inequities and injustices have been created and maintained, and fought against throughout history. In addition, criticality develops a critical praxis, or a problem posing education that builds curiosity through a questioning of the world. This replaces what Freire (1970) called the banking education, where information from the educator is deposited into the compliant, submissive student. Instead, the problem-posing educator facilitates learning through real-world experiences and a critical praxis involving dialogue and curiosity. "The role of the educator is to challenge the learner's naïve curiosity in order that they can both share criticalness, affirming itself, and unveiling hidden truths" (Freire, 1997, p. 52). For Freire (1997), criticality is created through dialogue and reflection. "Dialogue is full of curiosity and unrest., ... presupposes maturity, a spirit of adventure, confidence in questioning, and seriousness of providing answers" (p. 5). Dialogue is a critical posture of communication that goes past a scientific position of the world and stimulates curiosity. In addition, reflection is needed to form an understanding of historical criticality. Freire (1974) stated that reflection is a prerequisite, which enables people to "reflect upon themselves, their responsibilities, and their role in the new cultural climate-indeed to reflect on their very power of reflection., ... that would mean an increased capacity for choice" (p. 13). Thus, criticality requires critical thinking skills with a gained awareness in social and historical consciousness through problem posing, dialogue, curiosity, and reflection. 
EXAMINING TEACHERS' PARADIGM AND PEDAGOGY IN MANIFESTING CRITICAL HOPE IN LOW-INCOME MIDDLE SCHOOL

Without criticality, students are trained to adapt without protest and to continue the silence, without exposure to the possibility of the negation of social injustices (Giroux, 2011). It is a way of keeping society tamed as "Hope [is] pulverized in the immobility of the crushing present, some sort of final stop beyond which nothing is possible" (Freire, 1997, p. 55). Therefore, educators must gain an understanding of the social and political nature of schooling and the role they play in shaping what knowledge students receive and are exposed to through criticality (Giroux, 2011).

On social consciousness there is a gained awareness of the social and historical problems of the world, as people learn to help themselves though critical confrontation of their problems and become agents of their own liberation. Freire (1974) described a, "Consciousness about the world, which implies a consciousness about myself in the world, with it and with others" (p. 11). Thus, without a critical consciousness of the world and their responsibility in the world, men and women are not able to integrate themselves into the struggle in order to gain experiences marked by intense change and active participation (Freire, 1974).

On sense of community, Freire (1997) stated the struggle for hope becomes impermanent and intensified "when one realizes it is not a solitary struggle" (p. 59). As previously stated, the unification of those who are facing oppression brings hope. In addition, in bell hooks' $A$ Pedagogy of Hope (2003) she discussed the importance of creating community within the classroom environment for children of poverty. "In them I work to recover our collective awareness of the spirit of community that is always present when we are truly teaching and learning" (hooks, 2003, p. xv). Thus, a sense of community increases the will to hope and the will to struggle. 
EXAMINING TEACHERS' PARADIGM AND PEDAGOGY IN MANIFESTING CRITICAL HOPE IN LOW-INCOME MIDDLE SCHOOL

On caring relationships, Buber (1970 as cited in Noddings, 2017) stated, "In the beginning is the relationship" (p. 69) demonstrating that before any learning can occur, a relationship based upon caring must be established. The ethics of care is founded upon the notion that education is a relational activity shaped by the interaction of both parties, the carer (the one providing the care) and cared for (the one receiving the care) (Noddings, 2017). The role of the carer is to be attentive, listening to the voice of the cared for through multimodal literacies including not limited to body and facial gestures, and language both written and spoken (Kalantzis, Cope, Chan, \& Dalley-Trim, 2016) leading the carer to a reflection of feeling and perhaps an understanding of what the cared for may be experiencing (Noddings, 2017). The cared for responds to the relationship and supports it by smiling, or providing notions of affirmation that the relationship has been received, creating reciprocal feelings assisting to sustain that caring relationship (Noddings, 2017). In addition, Freire (1997) stated the importance of educational relationships as a "need for relational experience on the level of existence and interactions of the living" (p. 59). Thus, caring relationships are the foundations laid, which pave the way for one to hope.

On social and cultural capital, Freire (1997) stated that human nature is socially and historically constituted. This is a Marxist (Gee, 2013) view on human nature as some groups in the world are seen as naturally inferior in biological terms to other groups, and explains why dehumanizing practices occur across the globe. Bourdieu's (1986) political economy of symbolic power introduced relations between symbolic and material dimensions of social life. Bourdieu (1991) built upon social economic structures introduced by Marx and Engels in the Manifesto of the Communist Party (1848) and dove deeper into the symbolic dimensions of political economy. In this economy, he separated himself from the view of Marxism through 
EXAMINING TEACHERS' PARADIGM AND PEDAGOGY IN MANIFESTING CRITICAL HOPE IN LOW-INCOME MIDDLE SCHOOL

three distinct cultural practices of symbolic power/symbolic interests, capital power, and symbolic violence. These three cultural practices have autonomy, but they also intersect, and overlap each other within the social realm. The knowledge of social and cultural capital is imperative because it provides recognition of the skills and funds of knowledge (Moll \& Gonzalez, 2004) that marginalized students bring into the classroom and replaces the elitist deficit thinking that embodies a notion that lower classes "require medicine" or ailment of their disease (Freire, 1974). As, “all learners construct knowledge from an inner scaffolding [made up] of their individual and social experiences, emotions, will, aptitudes, beliefs, values, selfawareness, purpose, and more" (Senge, 2012, p. 692). Thus, it is important for educators to recognize the social and cultural capital that their students bring with them into the classroom as a means of hopeful, humanizing, and transformative practice.

On democratic advocacy, Freire (1997) stated, "What makes me hopeful is not so much the certainty of the find, but my movement in the search" (p. 59). Democratic advocacy is defined as those who gain social and historical consciousness and choose to engage in the struggle towards liberation. The awareness brings a lack of satisfaction in their world and of their place in the world and encourages action, as the oppressed are "no longer satisfied to watch, they want to participate" (Freire, 1974, p. 11). This democratic advocacy is deemed as a threat to the dominant culture because they become actively involved in their emancipation. "In this strange interpretation of democracy, health is synonymous with popular silence and inaction" (Freire, 1974, p. 11). Educators can empower students through providing opportunities that develop a critical social and historical consciousness of the ways people are systemically oppressed in society. "It can provide the power for people to learn and grow even when their situations or environments are disempowering" (Senge, 2012, p. 700). Thus, democratic 
EXAMINING TEACHERS' PARADIGM AND PEDAGOGY IN MANIFESTING CRITICAL HOPE IN LOW-INCOME MIDDLE SCHOOL

advocacy is needed in order to produce and sustain hope within the struggle involved in the transformation of society.

On humanization, Freire (1970) discusses historically how this continues to be humanity's central problem and it must be addressed. However, the "concern for humanization leads at once to the recognition of dehumanization" (Freire, 1994, p.45), as one cannot begin to address humanization (Freire, 1970), without a critical, social, and historical consciousness that generates an awareness of how dehumanization exists in society through injustice acts such as oppression, exploitation, violence, racism, sexism, and classism. In addition, lack of recognition or misrecognition are also forms of oppression as, "Due recognition is not just a courtesy, but a vital human need" (Fraser, 1997, p. 14).

Dehumanization is pervasive and destructive to both the oppressed and the oppressor because "it not only marks those whose humanity has been stolen, but also (though in a different way) those who have stolen it" (Freire, 1970, p.45). Freire (1970) discusses the "great humanist and historical task of the oppressed is to liberate themselves and their oppressors" (p. 46). Liberation can only come through power that is brought forth internally from the oppressed out of a "true generosity" that fights to destroy oppression with selfless acts of love (Freire, 1970). In addition, oppression must be alleviated through the integration of humanizing practices that provide freedom, criticality, praxis, and autonomy. These humanizing practices enable the oppressed with the ability to reason in their world with criticality, praxis, and provide the choice to engage in the struggle in order to transform society and embrace their full humanity. Thus, humanizing practices are necessary acts that bring restoration to both the oppressed and the oppressor whose humanities have been distorted. 
EXAMINING TEACHERS' PARADIGM AND PEDAGOGY IN MANIFESTING CRITICAL HOPE IN LOW-INCOME MIDDLE SCHOOL

\section{Hope Theory}

Secondly, a discussion on the hope theory that was used to formulate "Critical Hope" will take place. The hope theory is a multidimensional construct consisting of three intersecting elements of goals, pathways, and agency. Hope is defined as, "a positive motivational state derived from a sense of successful agency and pathways' (Edwards et al., 2007, p. 83).

Educational research states that motivation includes four components: sense of belonging, autonomy, meaningfulness, and competency (Turner, Christenson, Kackar-Cam, Trucano, \& Fulmer, 2014). This means that classroom instruction must connect with students in these four components to increase student motivation. The positive motivated state is derived from a sense of successful agency, meaning that there has been success in the present, or in the past, which manifests in a personal belief they will be successful in the future. Agency was defined by Snyder (2000 as cited in Edwards et al., 2007) as, "The motivational component to propel people along their imagined routes to goals, ... also known as willpower, agency reflects the perceived ability to initiate and sustain movement toward a goal" (p. 84). This demonstrates that there is a cognitive analysis of one's ability to succeed. Agency looks at all the options, and considers if success is possible. When barriers arise, agency reflects a determination to meet the desired goal, and depending on one's level of hope the thoughts are either positive or negative in the form of "I can do this," or "I can't do this (Snyder, 1994b)" (as cited in Edwards et al., 2007, p. 84). The hopeless students are categorized as unmotivated, lacking will, apathetic, and having a belief that they cannot succeed.

Hope theory has been empirically used in medical research demonstrating that those who have more hope often heal faster than those who do not (Lopez, 2013). Hope has also been the notion of the School of Public Health as a "major break through in thinking., ... demonstrating 
that all diseases in the world are well correlated with social class" (Duncan-Andrade, 2009, p. 184). This research demonstrated that social class unequally distributes hope, just as health is unequally distributed along the social grain (Duncan-Andrade, 2009). Simply put, those who have high levels of hope often got sick less compared to those who have low levels of hope. It "is now thought of as one of the most-if not the most-significant contributors to poor health" (Duncan-Andrade, 2009, p. 185). Thus, research has demonstrated that the inequality of hope is making those positioned on the lower end of society sick (Duncan-Andrade, 2009). Because minimal research has been done in education relating to hope, the current study aims to add knowledge on the manifestations of Critical Hope in teachers' paradigm and pedagogy.

\section{Pedagogy of Hope}

The current study has been influenced by the seminal work of Paulo Freire on hope. In his book, Pedagogy of Hope, Freire (1994) explained the results of oppression in the peasants of Brazil, and the importance for people to be educators of hope for those who have been oppressed. He stated, "Just to hope, is to hope in vain" (1994, p. 2). This means that the feeling of hope is not enough to sustain; there must also be a belief that change is possible because of a visualization of the path one can take to create that change. When one hopes and does not do anything about their goals, it turns into hopelessness and despair. As a result, an actionless person, who lives inside the struggle, turns into a hopeless person. Freire (1994) stated, "Hopelessness and despair are both the consequence and the cause of inaction or immobilism" (p. 2). Thus, hope must involve action, and the more that one hopes and acts upon their hope, their belief strengthens because of the learned practice and exercising of hope. 
EXAMINING TEACHERS' PARADIGM AND PEDAGOGY IN MANIFESTING CRITICAL HOPE IN LOW-INCOME MIDDLE SCHOOL

\section{Critical Hope}

As previously described in my conceptual framework, Critical Hope contains the elements of the traditions of critical theories that include optimism, ethics, criticality, social and historical consciousness, sense of community, caring relationships, knowledge of prior experiences through social and cultural capital, democratic advocacy, and humanizing practices, and the hope theory that specifies goal directed thinking, with navigating pathways, and agency. This study will examine manifestations of Critical Hope in teachers' paradigm that influence their pedagogy and classroom practices. In this study, evidence of hope will be observed and identified in: (1) teachers' vocabulary expressions and written directions that connect, connote, or imply valuing and trusting optimistic aspirations to succeed, (2) teachers' written tasks and assignments that prompt critical thinking and aspirations to be caring, and (3) teachers' lesson plans that may elicit a sense of community and humanizing practices.

In the article, Note to Educators: Hope Required when Growing Roses in Concrete, Duncan-Andrade (2009) conceptualized Critical Hope to describe three elements of educational practices that can build true hope in urban schools, namely, material hope, socratic hope, and audacious hope. He started by discussing the three forms of false hope found in educational practices and beliefs: hokey hope, mythical hope, and hope deferred which have decimated hope for children in urban schools and have been “an assault on hope, particularly in our nation's urban centers ... including disinvestment in schools and overinvestment in prisons." (DuncanAndrade, 2009, p. 182).

According to Duncan-Andrade (2009), hokey hope is the notion that all are able to obtain, the ability to pull yourself up by the bootstrap, and go out in the world to live the American Dream. Mythical hope perpetrates that we live in a color-blind society by celebrating 
EXAMINING TEACHERS' PARADIGM AND PEDAGOGY IN MANIFESTING CRITICAL HOPE IN LOW-INCOME MIDDLE SCHOOL

the individual exceptions that have been able to make it out of the poverty cycle. Lastly, educators have formulated hope deferred (Duncan-Andrade, 2009) as a politics of despair by blaming the system for the conditions and challenges and removing themselves from the responsibility of manifesting forms of pedagogical transformation within that community of students.

Duncan-Andrade (2009) based his concept of Critical Hope upon Freire's (1997) pedagogy of hope and stated, "Paulo Freire (1997) described this kind of hope as an, 'ontological need,' especially in the lives and pedagogy of educators working in communities where forms of social misery seem to have taken up permanent residence" (Duncan-Andrade, 2009, p. 182). This relates to Rawlinson's (2011) scourge of poverty as the social misery a child experiences impacts their behavior, social ability, and belief in their future. In addition, according to S. Leonard Syme (2004, as cited in Duncan-Andrade, 2009) used a definition of hope that stated a "control of destiny, an actively present sense of agency to manage the immediate stressors of one's daily life" (p. 184). He provided the use of Critical Hope as a way to combat these false hopes by rejecting "cheap American optimism (West, 2008, p. 41)” (Duncan-Andrade, 2009, p. 185), but rather being committed to engage actively in the struggle.

Subsequently, Duncan-Andrade (2009) described three elements of Critical Hope. Material hope refers to educators cultivating a sense of control through quality teaching that produces outcomes pertaining to student growth. Socratic hope refers to teachers and students examining their lives and pain within the injustices in society and validating those feelings, which create a righteous indignation and an understanding that they are in the fight together (Duncan-Andrade, 2009). Audacious hope is manifested in two ways. The first way is through both educators and students sharing their burdens regarding the inequitable suffering they 
EXAMINING TEACHERS' PARADIGM AND PEDAGOGY IN MANIFESTING CRITICAL HOPE IN LOW-INCOME MIDDLE SCHOOL

experience, manifesting a humanizing hope for healing their pain through acknowledgement and deep caring relationships (Duncan-Andrade, 2009). The second way is to reinsert hope through the mainstream discourse through transformative educational principles and effective teachers. Thus, Duncan-Andrade implied that there is a need to transform the pendulum in educational practices and beliefs through a notion of Critical Hope in urban schools.

\section{Adding to the Knowledge of Critical Hope}

The current dissertation is an attempt to build upon Freire's (1994) pedagogy of Critical Hope and broaden Duncan-Andrade's (2009) conception of Critical Hope by incorporating the elements of optimism, ethics, criticality, social consciousness, sense of community, caring relationships, knowledge of social and cultural capital, democratic advocacy, and humanizing practices which are present in the foundational literature on the traditions of critical theories shown and discussed above in the current study's conceptual framework. Along with the elements of Critical Hope, Duncan-Andrade's (2009) conception is also broadened by employing the hope theory's construct found in the empirical work of Edwards et al. (2007) that includes goals, pathways, and agency.

In this dissertation, Critical Hope is differentiated from the common use of the word hope, which is commonly defined as an emotion, or a feeling that changes based on one's circumstances. "Critical Hope" is deeper than our fickle emotions. For the purpose of this study, hope is defined to be an internal belief that changes are possible, driven by a visualized trajectory leading toward the goal (Lalas, Charest, Strikwerda, \& Ordaz, 2019). In this definition, simply having a feeling of hope does not create hope. Hope must be accompanied with the action. The current research aimed to examine the manifestations of "Critical Hope" in teacher's paradigm and pedagogy through evidence of hope as observed and identified in: (1) teachers' vocabulary 
EXAMINING TEACHERS' PARADIGM AND PEDAGOGY IN MANIFESTING CRITICAL HOPE IN LOW-INCOME MIDDLE SCHOOL

expressions and written directions that connect, connote, or imply valuing and trusting optimistic aspirations to succeed, (2) teachers' written tasks and assignments that prompt critical thinking and aspirations to be caring, and (3) teachers' lesson plans that may elicit a sense of community and humanizing practices.

\section{Paradigm}

Henry Giroux (2011) discussed how educators' paradigm encompass their worldview and belief of their position in the world based on their prior experiences, their perceptions, and is reinforced politically through the education they received. According to Kuhn (1962), "A paradigm refers to the shared images, assumptions, and practices that characterize a community of scholars in a given field" (p. 80). The concept of paradigm is important because it frames and guides the practitioner's work, and is related to the social and political values in the larger society as a whole (Giroux, 2011).

In addition, paradigm is the genesis, the development, and the history behind a person's worldview and belief system (Kuhn, 1962). "Educational workers in public education are not only born into a specific historical context; they embody its history in varying ways both as a state of consciousness and as a sediment experience, as a felt reality" (Giroux, 2011, p. 32). It is important for educators to think critically, bringing the subconscious to the forefront of their conscious in regards to their paradigm and meditate upon how it has been shaped and constructed historically, politically, socially, and culturally (Giroux, 2011) through their interactions within the world (Kuhn, 1962). As paradigm and pedagogy intercept, "it is crucial to recognize that pedagogy has less to do with language of technique and methodology, than it does with issues of political power" (Giroux, 2011, p. 71). As a result, educators are either 
EXAMINING TEACHERS' PARADIGM AND PEDAGOGY IN MANIFESTING CRITICAL HOPE IN LOW-INCOME MIDDLE SCHOOL

reproducing history by being a product of it, or becoming aware of their place in their world in order to change history based upon praxis, reflection, and action (Giroux, 2011; Saltman, 2018).

Henry Giroux (2011) discussed how educators should meditate upon questions and reflections that analyze what viewpoint they embody, to discover if they are in support of the dominating view, or in opposition of it. For example: "What interest do my personal assumptions serve and what are the consequences of these assumptions? Are my assumptions creating inequalities within my work sphere? What are the material and intellectual forces that sustain these assumptions and their corresponding paradigm?" (Giroux, 2011, p. 33).

Consequently, it is imperative for educators to become aware and discover how one's paradigm shapes their view of knowledge that is delivered into the classroom through pedagogical practices and experiences their students receive.

Critical theorists have expounded upon the notion of paradigm in many forms. Foundationally, Kuhn (1962) single handedly changed the meaning of the word paradigm by evolving the connected connotations that were used previously relating to the word, before his time. As a result, the word paradigm continues to evolve. In Aristotle's book Rhetoric (4th century BC) he discussed the art of persuasion and used the word paradigm to describe the discussion between two parties, an orator and an audience who share a great many beliefs that need to be stated (Kuhn, 1962). Aristotle's use of the word paradigm meant an exemplar of the dispute between the two parties based off of the views and beliefs of those involved in the discussion (Kuhn, 1962). Thus, for Aristotle, the word paradigm evolved into the word exemplar, where a standard model was to be followed for the specific context (Kuhn, 1962).

The primary use for the word paradigm was related to grammar and was also used as a metaphor (Kuhn, 1962). In Germany in 1930s, the word paradigm was commonly used in the 
EXAMINING TEACHERS' PARADIGM AND PEDAGOGY IN MANIFESTING CRITICAL HOPE IN LOW-INCOME MIDDLE SCHOOL

philosophical writings of the Vienna Circle (Kuhn, 1962, p. xx). It then emerged in America as a philosophy of science and appeared many times in Viennese Ludwig Wittgenstein's (1953 as cited in Kuhn, 1962) book Philosophical Investigations using the word paradigm related to grammar and language games. Eventually, Kuhn (1962) evolved the word to mean a sharing of intellectual attitudes and problems that is legitimized by what the community does and believes based off of extensive research that was done on the phenomena. In Kuhn's (1962) book Second Thoughts on Paradigm he stated that paradigm refers to, "the achievements served as exemplars of what to do, the kinds of questions to ask, successful application, and exemplary observations and experiences" (p. xxii). Thus, paradigms were considered the normalcy that legitimized the problems and puzzles within the scientific community, during that time (Kuhn, 1962).

As Kuhn (1962) continued to discover the connotation behind the word paradigm, he discovered a new word, paradigm shift, which is a trendy word commonly used today in educational practice. According to Kuhn, a paradigm shift occurs when the methods legitimized by the paradigm go wrong, becoming counter to what was expected to happen. These contradictory occurrences are defined as anomalies, or a crisis that is contrary to the established order that results and persists as a historical event, resulting in a redirection of research, and creating a means for a new paradigm (Kuhn, 1962). A paradigm shift begins, as a solution to the new crisis is needed, resulting in an extraordinary amount of new research where new ideas arise, with new methods, a new theory, and a new worldview which creates a revolution. The revolution encompasses a paradigm shift that formulates a new world order with words that express the changed thoughts, the different view of the world, the different feelings for how it works, the different phenomena and puzzling of difficulties, and the new ways of interacting 
EXAMINING TEACHERS' PARADIGM AND PEDAGOGY IN MANIFESTING CRITICAL HOPE IN LOW-INCOME MIDDLE SCHOOL

(Kuhn, 1962). As a result of the revolution, there is a change in meaning within the researchers themselves, which is demonstrated, by a change in theory.

\section{Meaning-Making and Designing}

Paradigm shift also relates to the meaning-making process and the designing process in education. The meaning-making process is a multi-literacies approach involving three fluid capacities that include representation, communication, and interpretation. Representation is what a person embodies: their background knowledge, their experiences, interests, views, and the way they make sense of the world. Communication involves the interactions with others and their individual representation, as a message is being communicated. "Communication is a reciprocal thing, in which one person's meaning-action prompts another person's representation, and probably also a communication from them" (Kalantzis et al., 2016, p. 217). Interpretation involves a transformation in the person as their representation combines with the communicated meanings that they are hearing, and results in an interpretation as they are making sense of what they hear. Thus, meaning-making is a social activity involving contact and communication (Dewey, 1938) as information that is communicated mixes with their set of lenses, is interpreted, and becomes their own knowledge.

The designing process builds upon the meaning-making process as the meaning-maker does not simply copy down or repeat was being communicated, but rather creates a new design (Kalantzis et al., 2016). As an expression of his or her own voice, "the meaning maker always creates a new design, a design like no other ever made before" (Kalantzis et al., 2016, p. 222). The designing process puts creativity and imagination at the center and involves design, designing, and redesign (Kalantzis et al., 2016, p. 222). Designs are available resources and expressive materials that can be used to make meaning and be reworked to create a new message 
such as artifacts, tools, and communication. Designing is when the meaning-making work occurs as the available resources are reconstructed for the purposes of representation and communication. Redesign is the newly created design of meaning that leaves the designer and the world transformed (Kalantzis et al., 2016, p. 222). Once a person reaches the process of redesign, that is where true learning occurs (Kalantzis et al., 2016). Kalantzis et al (2016) asserted, "this process of transformation is the essence of learning" (p. 224). Thus, the designing process is a theory of learning involving a paradigm shift as the world is transformed including the beliefs, values, experiences, and knowledge all of which are embodied in an individual's representation.

\section{Individual and Institutional Paradigm}

As Kuhn (1962) struggled to understand the depth and the various meanings behind the use of the word paradigm, he realized that there were two generalizable uses of the word: global and local. For Kuhn, global referred to the shared ideas in a community, the symbolic generalizations that bind the community of a particular discipline together, and what would cause them to tear apart. "This is a fundamental sociological question to be asked of any identified group, large or small, be it political, religious, ethnic, .. .what keeps this group together?" (Kuhn, 1962, p. xxiv). For the purpose of this dissertation, I am building upon Kuhn's use of global paradigm, to be named specifically as an "institutionalized paradigm," which is defined as an encompassing worldview, professional ideology, the position of education, politics, social, and historical content of a particular discipline that is directed into society and assists in the reproduction of societal systems and norms through social systems such as churches, colleges, media, communities, and families. 
EXAMINING TEACHERS' PARADIGM AND PEDAGOGY IN MANIFESTING CRITICAL HOPE IN LOW-INCOME MIDDLE SCHOOL

The second use of the word paradigm according to Kuhn (1962) is local which refers to various types of exemplars that serve as models for future practices that is legitimized by the research and the methods practiced by a scientific community. In this way, the achievement itself serves as the local paradigm (Kuhn, 1962). The reality is that the global, or institutionalized paradigm influences the local paradigm. Thus, for the purpose of this dissertation, I am building upon Kuhn's use of the word local paradigm to include one's “individual paradigm" because it influences ones identity, ideology, and individuality in every aspect of life. As one participates in a particular paradigm, he or she becomes socialized as their experiences, beliefs, behaviors, values, and feelings are shaped by the worldview they are situated in (Kuhn, 1962; see also Johnson, 2007) ultimately impacting their individual paradigm. According to Johnson (2007), "We also have to understand how they participate in particular kinds of social systems, how this participation shapes their behavior, and the consequences it produces" (p. 78). Thus, in order to understand a particular paradigm, we have to look at both the institutionalized paradigm and the individual paradigm of people who participate inside of it.

\section{Categories of Difference and Inequity}

When examining the prevalent achievement gap that exists between low-income students and affluent students as a measure of academic failure or success, one must also examine the "non-school factors" or "out-of-school factors" (Berliner, 2005). Berliner (2005) discussed the inequality that is found in areas of poverty as systems that are not created by educators themselves, but rather oppressive systems from policies and practices that are reinforced by the educational systems providing privilege to some at the expense of the majority. It is important that educators recognize these out-of-school factors that assist in perpetrating the continuum of inequities in order to bring hope to those who face them on a daily basis. There are categories of 
difference found in the context of race, economics, culture, gender, and sexual orientation as well as the context of language, abilities, and disabilities (Johnson, 2007). In addition, there are systems in place that provide privilege for one group over another group, which assists in the continual oppressive practices for those who are not privileged (Johnson, 2007). All these contexts of learning must be considered in providing the explanation of why inequality of education for student learning exists and what oppressive systemic practices continue those inequalities and how educators can equitable provide what student need in order to be successful, by learning who there students are, how they learn, and what motivates and facilitates their engagement (Lalas, et al., 2016).

We want equality but because of the different contexts or categories of difference among students, inequality happens. I believe that inequality is inevitable and how the educational system addresses these categories of differences may be valid factors in explaining why students perform differently in doing their academic tasks. Thus, we need to consider the impact of differences and other out-of-school factors on student achievement. If we do not address the inevitable inequalities, inequities are bound to occur.

This leads me to a discussion on what I am coining to be "institutionalized deficit paradigm" and "internalized deficit paradigm." Institutionalized deficit paradigm are the systemic oppressive structures that exist in society in framing a deficit worldview and ideology for diverse children of poverty based off of categories of difference and privilege, and assists in the reproduction of societal systems through social structures such as churches, colleges, media, communities, and families. Institutionalized deficit paradigm informs and influences an individual, as they internalize the deficit worldview and thinking pertaining to diverse children of poverty, which may impact an individual's beliefs, assumptions, and attitudes that "not only 
EXAMINING TEACHERS' PARADIGM AND PEDAGOGY IN MANIFESTING CRITICAL HOPE IN LOW-INCOME MIDDLE SCHOOL

guide our perceptions about the phenomenon, but also direct our everyday schooling actions/activities, such as instructional practices, curriculum, and types of assessment used" (Flores, Strikwerda, \& Ordaz, 2019, p. 10). Thus, the institutionalized deficit paradigm influences, shapes, and reproduces an individual's internalized deficit paradigm which assists in reproducing the inequities found in oppressive social systems.

Therefore, I am proposing that we need a paradigm shift away from systemic oppressive thinking that reinforces and reproduces both institutionalized deficit paradigms and individual internalized deficit paradigms, or hopeless paradigm to an assets-based paradigm, or hopeful paradigm. This research asserts that educators of children of poverty may need to construct an alternate, assets-based paradigm and social practices of hope that may affect the consciousness and needs of their students which fosters critical thinking and social advocacy (Giroux, 2011). As research has proven time and time again that educators are the most important factor in determining student achievement, which is directly related to their belief in their students' abilities, the expectations that they set inside the classroom, and teacher preparation (Terada, 2019), it is imperative that "educators, especially teachers need to name and interrogate their deficit views of language use, culture, and learning in order to be at a place where they decide to transform them (Flores et al., 2019, p. 10)" as agents of hope for children of poverty.

\section{Pedagogy}

The research sought to determine whether or not a teacher's paradigm and pedagogy are reflective of their hopefulness or hopelessness about their students' abilities and future aspirations and their beliefs and expectations of what their students are capable of doing. This research asserts that educators of children of poverty may need to construct alternate paradigms and social practices of hope that may affect the consciousness and needs of their students, which 
EXAMINING TEACHERS' PARADIGM AND PEDAGOGY IN MANIFESTING CRITICAL HOPE IN LOW-INCOME MIDDLE SCHOOL

fosters critical thinking and social advocacy (Giroux, 2011). Pedagogy is not only found in the materials, techniques, and methodologies that is happening, it is also found in the political power driving the curriculum, and the educator who is implementing to knowledge. For example, initially Common Core was a political move as politicians passed policy on common core and then it was framed into a curriculum (Clayton, 2016). Pedagogy is broader and encompasses more than martialing direct instruction and lesson planning. Consequently, the combination of the elements of the traditions of critical theories and hope theory created the notion of Critical Hope that may play a vital role in informing teacher's paradigm and pedagogy in teachers of children of poverty.

\section{Student Engagement}

As this research examined, poverty can impact students' lives in various ways leading to hopelessness which can affect the students behavioral, social, and academic outcomes (Rawlinson, 2011). Since hope is defined as a "positive motivational state" where motivation is the internal factor and student engagement is the external factor. Thus, there is a need for educators to gain awareness on how hope impacts student engagement in children of poverty. Research showed that effective engagement is an indicator of academic success and can predict how students will perform in school (Reeve, 2012). Reeve (2012) defined engagement as the extent of a student's active involvement in a learning activity. Research suggested that without engagement, students are not learning (Reeve, 2012). Engagement can be observable through academic achievement and on-task behaviors, and unseen internally through cognitive connections leading to meaning making and affective aspects where students find value in what they are learning (Reschly \& Christenson, 2012). Cognitive engagement focuses on the student's perception (Reschly \& Christenson, 2012) how they feel and think about school, as well as their 
EXAMINING TEACHERS' PARADIGM AND PEDAGOGY IN MANIFESTING CRITICAL HOPE IN LOW-INCOME MIDDLE SCHOOL

intrinsic motivation, self-efficacy, and a positive self-perception (Mahatmya, Lehman, Matjasko, \& Farb, 2012). Affective engagement is an emotional response where involvement in activities occurs through feelings of worth or enjoyment (Finn \& Zimmer, 2012). Affective engagement establishes incentives for students to engage in school activities and to work harder to overcome challenging tasks, or to take risks (Finn \& Zimmer, 2012). Thus, engagement is

multidimensional (Reschly \& Christenson, 2012) and may be influenced by the manifestations of hope in teachers' paradigm and the pedagogy inside the classroom.

\section{Research Questions}

The pedagogy of "Critical Hope" this dissertation formulated emphasizes the use of empirical data through the hope construct and critical theories in education which operationalizes the elements of "Critical Hope" as goal-directed thinking, navigating pathways, agency, optimism, ethics, criticality, social and historical consciousness, sense of community, caring relationships, social and cultural capital, critical citizenry, democratic advocacy, and humanizing practices. Specifically, this study focused primarily on (1) teachers' language and behaviors of hope, (2) teachers' instructional delivery of hope, and (3) teachers' lesson planning of hope. In this study, Critical Hope is defined as the optimistic way of viewing and acting on the world from a critically historically conscious, socially and culturally situated perspective with the belief that inevitable change will inspire a sense of community, advocacy, liberation, and justice. Empirically, hope is defined as "a positive motivational state that is based on an interactively derived sense of successful agency and pathways" (Edwards et al., 2007, p. 83). In this study, hope is operationalized as: (1) teachers' vocabulary expressions and written directions that connect, connote, or imply valuing and trusting optimistic aspirations to succeed, (2) teachers' written tasks and assignments that prompt critical thinking and aspirations to be caring, (3) 
teachers' lesson plans that may elicit a sense of community and humanizing practices. The rationale is to give teachers in low-income middle school the forum to manifest their understanding of Critical Hope in their paradigm and pedagogy. A qualitative, methodological approach using narrative inquiry was selected to seek answers to the research questions used to study, understand, and reconstruct the lived experiences of teachers in the realm of practice while delving into conceptual exploration (Conle, 2001).

This research sought the answers to the following questions:

1. To what extents do the voices of teachers of low-income students reflect Critical Hope in their paradigm and pedagogy?

2. What themes related to teachers' language and behavior of hope, teachers' instructional delivery of hope, and teachers' lesson planning of hope emerge when observing and listening to teachers?

3. What recommendations for educators can be made to reflect the manifestations of “Critical Hope" in teachers' paradigm and pedagogy?

Question one explored how reflections of hope inform teacher's paradigm and pedagogy in low-income middle school classrooms. Specifically, the finding should draw a connection between hope and how that informs teacher paradigm and pedagogical practices in their lowincome middle school classrooms. Rawlinson (2011) connected the poverty mindset as a contributor to hopelessness that is manifested in behavioral, social, and academic outcomes of low-income students that may be perceived as students who are unmotivated and disengaged. Thus, teachers who have reflections of hope provide a connection between hopeful paradigm and pedagogical practices and their students' quests for success. 
EXAMINING TEACHERS' PARADIGM AND PEDAGOGY IN MANIFESTING CRITICAL HOPE IN LOW-INCOME MIDDLE SCHOOL

Question two aimed to shed light related to the themes that emerge in teachers' language and behavior of hope, teachers' instructional delivery of hope, and teachers' lesson planning of hope emerge when observing and listening to teachers of low-income students. Specifically, if teachers' voices reflect the realities and systemic inequities children of poverty face, as "equal treatment for children in unequal situations is not justice" (Chen \& Hahnel, 2017, p. 1). Thus, equitable remedies for children of poverty who face systemic inequities begin with teachers' recognition that unequal conditions occur and require equitable educational experiences and what the role of hope is in diminishing those inequities. In addition, themes emerged through classroom observations that revealed reflections of hope through teachers' paradigm and pedagogy allowing for connections between what the teachers express in their voices to what actions are displayed inside the classroom.

Question three allowed for recommendations to be made to teachers, administrators, and policymakers related to professional learning on "Critical Hope" and how it may impact student engagement for low-income middle school students. Pragmatically, the gathered information aims to be used as means of transforming inequities that children of poverty may face and creating more equitable conditions that may influence their student engagement.

The overarching goal of this dissertation was to determine how teachers manifest Critical Hope in their paradigm and pedagogy in low-income, middle school classrooms. Conceptually, teachers may reveal how their pedagogy and paradigm inform their desire for students to be hopeful in their language and behavior, instructional delivery, and lesson planning in performing school tasks. Theoretically, this study employed critical theories such as Freire's $(1994,1970)$ pedagogy of hope and other critical theories (Brookfield, 2005; Giroux, 2011; Saltman, 2018) including hope construct by Edwards et al. (2007) as the conceptual framework. Current 
EXAMINING TEACHERS' PARADIGM AND PEDAGOGY IN MANIFESTING CRITICAL HOPE IN LOW-INCOME MIDDLE SCHOOL

research demonstrated that students with high levels of hope engage in and persevere through tasks better and lead to higher achievement, while those with lower levels of hope disengage from school and have negative school outcomes (Edwards et al., 2007). Practically, this study gathered data manifesting practices related to Critical Hope by interviewing and observing teachers of low-income students through narrative inquiry, which may influence local school districts' professionals learning about the importance of highlighting hope for student engagement.

\section{Narrative Inquiry}

The research objective of this qualitative, narrative inquiry provided an in-depth investigation of the meaning given to experiences of participating teachers in order to reveal themes embedded in storytelling that may reflect hope and recognize what the needs of lowincome students are. Voice is used as an outward expression of lived experiences with our social environment (Bakhtin, 1981). Narrative inquiry describes the phenomenon of personal experiences and is rooted in a circular dimensional practice of inquiry and discovery (Conle, 2001). The aim was to bring understanding and clarity to a particular phenomenon through the telling of lived experiences (Connelly \& Clandinin, 1990). As the stories are told, and recalled from memory, themes emerged through theoretical exploration and interpretation of data sets (Conle, 2001).

Furthermore, Conle (2001) described that truth claims can be verified through theoretical discourse as narrative inquiry may assist with clarifying lived values and to determine what may be good for a particular society or culture. This demonstrates that narrative inquiry can serve to clarify lived experiences and be used as a method for change in a particular society. In this way, research relating to the experiences of teachers of low-income students becomes the 
phenomenon, or the story, and the inquiry with the use of open-ended questions and the participants voices used to construct these experiences becomes the method (Connelly \& Clandinin, 1990). Dewey (1938) explained that using the experiences of means of reflection becomes a method enabling people to connect their actions and the consequences of it. In using narrative inquiry, this dissertation sought to bring authentic teachers' voices to the forefront through storytelling so that they become a part of the educational literature on hope, pedagogy, and paradigm and their connection to student engagement

\section{Definition of Terms}

- $\quad$ Action- According to Bourdieu, actions are self-satisfying, self-vested, and self-interested relating to both unconscious and conscious actions, which are motivated by self, which leads to interests instead of a means end or a physical goal (Swartz, 1997).

- $\quad$ Affective Engagement- Affective engagement is a level of emotional response characterized by feelings of involvement in school as worth pursuing; it is the value placed on what they are learning (Finn \& Zimmer, 2012)

- $\quad$ Affirmative Solutions- Affirmative solutions refer to solutions that address inequalities without restructuring the systemic frameworks. It is a surfaced approach, similar to putting a Band-Aid on the problem (Fraser, 1997).

- $\quad$ Agentic Engagement- Agentic engagement is a student's ability to enrich their own learning experience by being active learners, through asking higher-leveled questions, and providing input in what lessons they would like to learn (Reeve, 2012).

- Agency- "Agency is the motivational component to propel people along their imagined routes toward goals, also known as willpower" (Edwards et al., 2007, p. 8). Agency reflects the perceived ability to initiate and sustain movement toward a goal based on the 
success they have experienced in the past and a vision of a path towards success in the future (Edwards et al., 2007).

- Assessment- Assessment, evaluation, and testing are defined as a tool of measurement, which is used to interpret data that has been gathered systematically as a determination of a subject's merit, and effective practice of instruction, by using criteria governed through a set of standards.

- Behavioral Engagement- Behavioral engagement focuses on behaviors exhibited, both positive and negative including attendance, graduation rates, grades, and disruptive behaviors (Mahatmya et al., 2012).

- Colorblindness- Colorblindness is the belief that people do not see color, only people, even though discrimination shows its face within society in many forms (Bonilla-Silva, 2017)

- Critical Hope- Critical Hope is defined as the optimistic way of viewing and acting on the world from a critically, historically conscious, socially and culturally situated perspective with a personal belief that inevitable change will inspire a sense of community, advocacy, liberation, and justice.

- Critical Praxis- Critical Praxis is a cycle of awareness involving five steps including identifying a problem, analysis of the problem, creating a plan for action, implementation of the plan, reflection upon the action, which leads to more action (Duncan-Andrade \& Morrell, 2008).

- $\quad$ Critical Theory- Critical theory emphasizes a scholarly approach that explores many dimensions including, history, policy, culture, and ideology; it is a complex process that 
takes on new knowledge with the purpose of understanding the history, political, and social movements behind it to create change in society (Sensoy \& DiAngelo, 2012).

- Critical Theory of Recognition- Critical Theory of recognition is "one that identifies and defends only those versions of the cultural politics of difference that can be combined with the social politics of equality" (Fraser, 1997, p. 12).

- Cognitive Engagement- Cognitive engagement is engagement that manifests mindful behavior, persistence, and metacognition; it includes the effort needed to comprehend complex thinking and the willingness to master difficult skills (Fredricks, Blumenfeld, \& Paris, 2004).

- Cultural Capital- Cultural capital is the education, culture, and traditions found in a person's life (Swartz, 1997).

- Dehumanization- Dehumanization refers to the effects of oppression in humanity, and results in internal feelings of unworthiness from both the oppressed and the oppressors. This occurs when humans take unjust actions towards another human that give rise to distorted notions that the oppressed are seen as less human (Freire, 1970).

- Equity- Equity is theorized as the relevant and appropriate instructional, curricular, and programmatic attempt to meet the needs of all students when they need them (Blankstein, Noguera, \& Kelly, 2016; Lalas, Charest, Strikwerda, \& Ordaz, 2019).

- Emotional Engagement- Emotional engagement refers to engagement that encompasses positive and negative reactions towards the institution including teachers, classmates, academics, and school and how it influences willingness to do the work (Fredricks et al., 2004). 
- Economic Power- Economic power includes the structures of material economy, meaning money and property (Swartz, 1997).

- Equalitarianism- Equalitarianism is the belief of equality for all people.

- $\quad$ Goals- "Goals are targets of mental action sequences" (Edwards et al., 2007, p. 84).

- $\quad$ Hope- Hope is an internal belief that change is possible, driven by a visualized trajectory leading towards the goal. Hope is a positive motivational state that is based on an interactively derived sense of successful agency and pathways" (Edwards et al., 2007, p. 83).

- Hope Cycle- There are two cycles of hope: positive and negative. The hope cycle is a continuous cycle derived by hope: hope breeds achievement, and achievement breeds opportunity, and opportunity breeds more hope. Thus, the continual cycle produces or reduces hope depending upon the success or failures one personally experiences.

- $\quad$ Hope Gap- Is the zone of difference representing sets of expressions and actions that reflect hope and hopelessness in humanity. The hope a student has for their life based upon their past experiences and inaction. Hope gap is demonstrated in those who are hopeful compared to those who are hopeless, which can be observed by lack of motivation and disengagement. Hopelessness creates a cycle of failure, lack of motivation, and lack of achievement. Contrarily, hope creates a cycle of success, motivation, perseverance, agency, and success.

- Hopelessness- Hopelessness is the lack of belief that change is possible which ultimately turns into despair and involves a lack of action or immobility.

- $\quad$ Hope Theory- The hope theory discusses how a belief in oneself produces a positive motivation that creates pathways and agency for students and adults to generate success. 
In the same way, the lack of hope creates a cycle of failure, lack of motivation, and lack of achievement.

- Hope Scale- The hope scale is a measurement used in adults and children that determines the correlation between one's hope and achievement (Edwards et al., 2007).

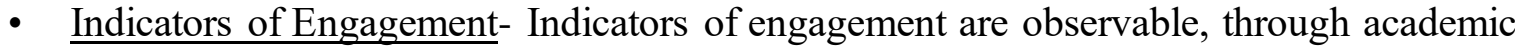
and behavioral activities and internal and affective domains, cognitively, and agentic, which leads to higher academic achievement (Reeve, 2012).

- Individual Paradigm- Individual paradigm is the belief of ones identity, ideology, and individuality that influences every aspect of life. As one participates in a particular paradigm, he or she becomes socialized as their experiences, beliefs, behaviors, values, and feelings are shaped by the worldview they are situated in Kuhn, 1962; see also Johnson, 2007) ultimately impacting their individual paradigm.

- Institutionalized Paradigm- Institutionalized paradigm is defined as an encompassing worldview, professional ideology, the position of education, politics, social, and historical content of a particular discipline that is directed into society and assists in the reproduction of societal systems and norms through social systems such as churches, colleges, media, communities, and families.

- Interest- Interest according to Bourdieu are both unconscious and conscious actions that are motivated by self, and provides the connection between economics in materialism and the symbolic economy of cultural and social capitalism (Swartz, 1997).

- Motivation- Motivation includes four components: Sense of belonging, autonomy, meaningfulness, and competency (Turner et al., 2014). 
- $\quad$ Official Poverty Measurement- The Official Poverty Measurement introduced in 1965 by Mollie Orshansky is a statistical methodological way of measuring poverty through thresholds for planning purposes. Poverty is determined by the capital one has acquired, divided by the amount of family members in that household.

- Oppression- Oppression refers to unjust systems, orders, behaviors, and attitudes towards humanity and can occur in the macro systems and the micro systems, and/or internally or externally, which leads to dehumanization, and gives rise to violence for both the oppressed and the oppressor (Freire, 1970).

- $\quad$ Pathways- "Pathways are ways that one can visualize achievement of goals through a perception that they are capable of imagining one or more routes toward success" (Edwards et al., 2007, p. 84).

- $\quad$ Poverty- Poverty is measured by the capital one has, and can be defined as the state of one who lacks a socially acceptable amount of money, or material possessions, to satisfy basic needs (Poverty, 2018).

- $\quad$ Poverty Blindness- Poverty blindness represents a systemic blindness towards the causes of poverty internally and externally representing educational policy, teacher pedagogy, and internalized oppression in the students, relating to the possibility of a broader spectrum. It is taken from the notion of culture blindness, a psychological term that is defined as the incapacity to comprehend how individuals in a different culture experience specific situations due to the systematic construction and alignment of viewpoints, outlooks, and morals of one's own society or culture (N., Pam, 2013).

- $\quad$ Self Efficacy- Self-efficacy is an internal, self-perceived competence level in one's abilities. 
- $\quad$ Social Capital- Social Capital refers to relationships and networks a person has solidified in their life (Swartz, 1997).

- Social Stratification- Social stratification refers to the concept that social groups are relationally positioned and ranked into hierarchy of unequal value and is used to justify unequal resources among groups (Sensoy \& DiAngelo, 2012).

- $\quad$ Student Engagement- Student engagement refers to the external, observable and internal expressions, and the active verb between the curriculum and actual learning (Skinner \& Pitzer, 2012). Engagement is multidimensional (Reschly \& Christenson, 2012) as it is the extent of a student's active involvement and investment in a learning activity (Reeve, 2012) including indicators of engagement that are observable, through academic and behavioral activities, affective, cognitive, and agentic domains.

- $\quad$ Supplemental Poverty Measurement- The Supplemental Poverty Measurement (SPM) is the new complex measurement that determines a statistical picture which includes additional items of tax payments, work expenses, government benefits, and all family resource estimates (Renwick, Short, Bishaw, \& Hokayem, 2012). The SPM also takes in account the differential geographical costs across the United States as it adjusts according to families' geographical location and differential need.

- Symbolic Capital- Symbolic capital refers to the legitimization one has in their social role through the eyes of others (Swartz, 1997).

- Symbolic Interests- Symbolic interests are the notion of how economic interests relate or translate into non-economic interests (Swartz, 1997).

- Transformative Solutions- Transformative solutions are remedies found through dismantling the current system and changing the root of the problem. This is not a 
EXAMINING TEACHERS' PARADIGM AND PEDAGOGY IN MANIFESTING CRITICAL HOPE IN LOW-INCOME MIDDLE SCHOOL

gradual change like affirmative but as Fraser (1997) stated it "apocalyptic change" (p. 23). This type of change is extremely hard to do in a society, especially when the change will ultimately affect the pockets of the dominant group.

\section{Recommendations}

The intent of this study was to secure and generate data to support the recommendations for educators and policy makers regarding professional learning on the issue of "Critical Hope" for teachers of low-income students in middle school. The goal was to give teachers of lowincome students a forum on hope as a topic through narrative inquiry that may influence local school districts' professionals learning about the importance of highlighting hope for student engagement leading to academic success. Consequently, this study yields stories related to hope that may inform teachers, administrators, and policy makers on how to work effectively with children of poverty whose voices and experiences have been traditionally and historically silenced, or disregarded in the current system of education.

\section{Staff Development}

Potential policy recommendations for staff development are important because they provide opportunities for continual growth and learning, improving skills, and knowledge of personnel (Webb \& Norton, 2009). No Child Left Behind (2002) developed new performance levels and provided initiatives to increase the development of effective pedagogy and technological skills. Professional development moves the staff forward towards student achievement and stated objectives of the organization (Webb \& Norton, 2009). Staff development is based off of motivation and theoretical notions of human behavior, which include the recognition that employees must be willing to devote time and energy into learning, they must feel what they are learning is necessary to improve in their personnel skills and knowledge, 
and they must be a part of a climate that fosters learning where they have autonomy to implement the needed changes (Webb \& Norton, 2009).

Web and Norton (2009) identified a framework related to professional development. The process involves five steps. They are the adoption of policy on staff development, create objectives and goals based off of the adopted policy, planning the methods of delivery, scheduling and delivering the activities to put the program in place, and evaluation of staff's progress (Webb \& Norton, 2009). The policy sets the guidelines and the objective of the staff development. It is the foundational process that staff development is built upon. It is important that the staff development matches the organizational goals. In addition, once the professional development occurs, there needs to be immediate application and implementation to find satisfaction in the learning process. Finally, a follow-up of evaluation must take place for refinement and reflection to deepen the learning and understanding (Webb \& Norton, 2009).

According to Webb and Norton (2009), staff development is based on motivation and theoretical notions of human behavior, which include three major purposes. First, is the recognition that employees must be willing to devote time and energy into learning, then they must feel what they are learning is necessary to improve in their personnel skills and knowledge related to competency and future advancements, and finally they must be a part of a climate that fosters learning where they have autonomy to implement the needed changes (Webb \& Norton, 2009). As a result, professional development is the driving force behind the movement of student achievement related to the established goals of the organization.

\section{Organization of Study}

This qualitative narrative study was organized into five chapters. Chapter One contained an overview of the plight of children of poverty, specifically leading to inequities in education 
EXAMINING TEACHERS' PARADIGM AND PEDAGOGY IN MANIFESTING CRITICAL HOPE IN LOW-INCOME MIDDLE SCHOOL

pertaining to access and academic success, the significance of this study, the conceptual framework used to guide the research, the research questions, and recommendations. Chapter Two includes a detailed literature review on poverty, educational inequities, paradigm and pedagogy, traditions of critical theories as foundational literature, the hope theory as empirical literature, how I connect Critical Hope through the traditions of critical theories and the hope theory, motivation and student engagement. Chapter Three includes a description of the methodology used for this qualitative study as well as the researcher's positionality, the setting, instrumentation, and data analysis procedure. Chapter Four includes a discussion of the process of data analysis, the findings from the qualitative data gathered, and contains the 10 narratives. Finally, Chapter Five includes a summary of the previous chapters, a discussion of the research questions, and recommendations for K-12 teachers, administrators, and policy makers, and future research.

\section{Conclusion}

Through an examination on the plight of children of poverty, a gained awareness occurs pertaining to equity and the inequities found in education. A social-justice perspective is needed to combat the inequitable relationship between systemic educational inequities and academic achievement for children of poverty. "A large part of social suffering stems from the poverty of people's relationship to the educational system, which not only shapes social destinies but also the image they have of their destiny" (Bourdieu, 1998, p. 43). The inequitable reality that children of poverty face is "there is no one in America, Black or Brown, White or Asian, rich or poor, who is untouched by the human and economic toll of America's vastly inequitable public school systems" (Flint, 2018, p. 28). Thus, an equitable conversation is needed in order to take a political stand diminishing inequities found in low-income schools to create change in the 
intersecting relationship between inequitable educational achievement and children of poverty as, "we cannot lose sight of the link between injustice and suffering" (North, 2008, p. 1200). Social-justice leaders must continue to question, critically analyze, and explore the relationship between dominants of power in society and consistent levels of poverty for low-income schools. 
EXAMINING TEACHERS' PARADIGM AND PEDAGOGY IN MANIFESTING CRITICAL HOPE IN LOW-INCOME MIDDLE SCHOOL

\section{Chapter 2. Literature Review}

This chapter is organized into a discussion of poverty, educational inequities, paradigm and pedagogy, traditions of critical theories as foundational literature, the hope theory as empirical literature, how I connect Critical Hope through the traditions of critical theories and the hope theory, and on engagement. The review of this research literature provided the formulation of my conceptual framework and how this framework guides my research questions and eventually directs the gathering of data and framing its analysis.

\section{Poverty}

Currently, poverty is measured in two ways. The first way is the Official Poverty Measurement introduced in 1965, by Mollie Orshansky who developed a statistical, methodological way of measuring poverty through thresholds for planning purposes. Poverty is determined by the capital one has acquired, divided by the amount of family members in that household (Poverty, 2017). The United States Census Bureau determines the poverty level throughout the country based upon guidelines set forth by the Office of Management and Budget's (OMB) Statistical Policy Directive 14 (Poverty, 2017). To determine poverty status, the Census Bureau uses a set of poverty thresholds, or a "yard stick” to reflect a family's need based upon income, family size, and age of the members, which is consistent across the US (Poverty, 2017). Thus, when a family's income is determined to be less than the directed poverty threshold, every family member is considered to be living in poverty.

The second way poverty is currently measured is through the Supplemental Poverty Measurement (SPM) developed in 2010 by an Interagency Technical working Group, based off of the recommendations of the 1995 National Academy of Sciences Report (NAS) titled Measuring Poverty: A New Approach and the massive research done on poverty over the past 20 
years (Renwick et al., 2012). Concerns arose regarding the adequacy of the existing poverty threshold measurement, and it was determined that a new measurement was needed to measure the effectiveness of federal policy on those living in poverty (Renwick et al., 2012). Since 1964, when President Johnson declared his "War on Poverty," policies have been put in place to combat poverty levels such as nutritional assistance, subsidized housing, and home energy assistance. The new measurement is a complex statistical picture, which includes additional items of tax payments, work expenses, government benefits, and all family resource estimates (Renwick et al., 2012). The SPM also takes in account the differential geographical costs across the United States. Differentially from the Official Poverty Measurement the SPM is not set in stone, but rather it adjusts according to families' geographical location and differential need. In addition, the Official Poverty threshold measurement does not include the measurement of noncash items, nor does it provide deductions of taxes, childcare, or medical expenses. Thus, with the policies that have been introduced since 1964, to try to combat the war on poverty, a new, more adequate measurement was needed to determine the effectiveness of these policies to be used alongside the Official Poverty Threshold Measurement. SPM is not meant to replace the Official Poverty Measurement, but rather to be used to disaggregate data in national sub populations to provide additional statistical data to help bring understanding to the diverse levels of poverty in the United States (Short, 2011; see Figure 2). 


\begin{tabular}{|c|c|c|}
\hline \multicolumn{3}{|c|}{ Poverty Measure Concepts: Official and Supplemental } \\
\hline & Official Poverty Measure & Supplemental Poverty Measure \\
\hline $\begin{array}{l}\text { Measurement } \\
\text { Units }\end{array}$ & $\begin{array}{l}\text { Families (individuals } \\
\text { related by birth, marriage } \\
\text { or adoption) or unrelated } \\
\text { individuals }\end{array}$ & $\begin{array}{l}\text { Resource units (official family definition } \\
\text { plus any coresident unrelated children, } \\
\text { foster children, unmarried partners and } \\
\text { their relatives) or unrelated individuals } \\
\text { (who are not otherwise included in the } \\
\text { family definition) }\end{array}$ \\
\hline $\begin{array}{l}\text { Poverty } \\
\text { Thresholds }\end{array}$ & $\begin{array}{l}\text { Three times the cost of a } \\
\text { minimum food diet in } \\
1963\end{array}$ & $\begin{array}{l}\text { Based on expenditures of food, clothing, } \\
\text { shelter and utilities - FCSU }\end{array}$ \\
\hline $\begin{array}{l}\text { Threshold } \\
\text { Adjustments }\end{array}$ & $\begin{array}{l}\text { Vary by family size, } \\
\text { composition and age of } \\
\text { householder }\end{array}$ & $\begin{array}{l}\text { Vary by family size and composition, as } \\
\text { well as geographic adjustments for } \\
\text { differences in housing costs by tenure }\end{array}$ \\
\hline $\begin{array}{l}\text { Updating } \\
\text { Thresholds }\end{array}$ & $\begin{array}{l}\text { Consumer Price Index: all } \\
\text { items }\end{array}$ & $\begin{array}{c}\text { Five-year moving average of } \\
\text { expenditures on FCSU }\end{array}$ \\
\hline $\begin{array}{l}\text { Resource } \\
\text { Measure }\end{array}$ & $\begin{array}{l}\text { Gross before-tax cash } \\
\text { income }\end{array}$ & $\begin{array}{l}\text { Sum of cash income, plus noncash } \\
\text { benefits that families can use to meet } \\
\text { their FCSU needs, minus taxes (or plus } \\
\text { tax credits), minus work expenses, } \\
\text { medical expenses and child support paid } \\
\text { to another household }\end{array}$ \\
\hline
\end{tabular}

(Fox, 2017, The SupplementalPoverty Measure, 2016, p. 2)

Figure 2: Official and Supplemental Poverty Measures

\section{What is the Current National Poverty Level?}

The latest data from U. S. Census Bureau (2018) used both the Official Poverty

Measurement and the SPM measurement. Since the War on poverty was declared over 50 years ago, The United States tax payers have spent over $\$ 22$ trillion dollars, not including Social Security or Medicaid, which is a significant amount of expenditures producing minimal decrease in the poverty thresholds (Rector \& Sheffield, 2011). In 1964, the Official National Poverty Level was $17.3 \%$. The most recent report showed the National Official Poverty Rate at $12.7 \%$ and the SPM poverty rate was $14.0 \%$ (Fox, 2017). These numbers show a national decrease in poverty. However, if you look closely at the lesser number of $12.7 \%$, that is an estimated 46 
million people in our nation who are currently living in poverty. In addition, depending on the US economy, the poverty rate fluctuates from year to year. Through an analysis of the poverty threshold timeline, one can determine that the poverty level has been fairly steady over the past 50 years (Rector $\&$ Sheffield, 2011). In addition, these numbers do not take into account the millions of Americans who straddle the poverty line, where getting sick and losing a job can plunge an entire family into poverty overnight. (see Figure 3)

CHART1

Poverty Rate, 1947-2012

PERCENTAGE OF INDIVIDUALS WHO WERE POOR BY THE OFFICIAL POVERTY STANDARD

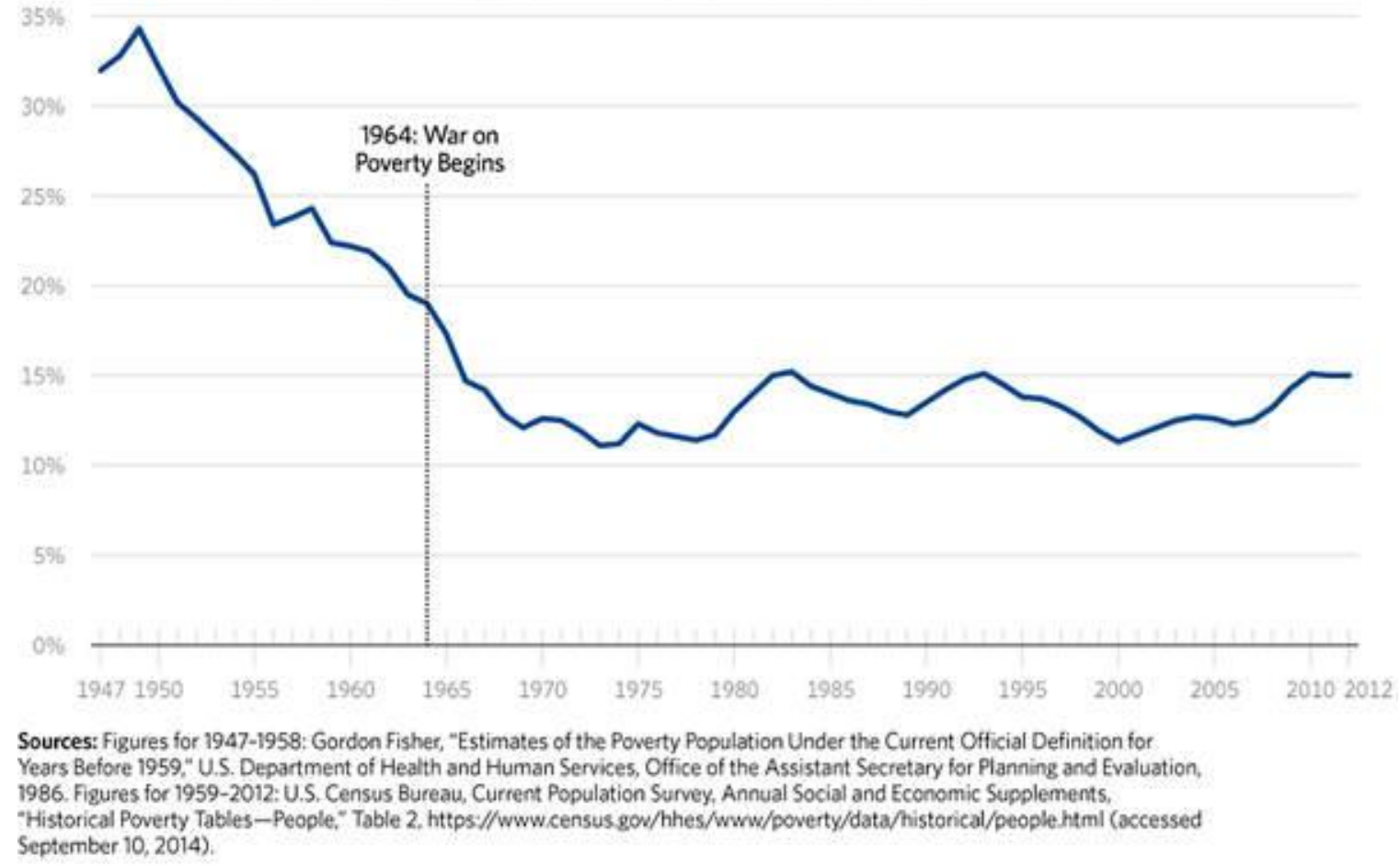

$862955 \mathbf{2}$ heritage org

Source: (Rector \& Sheffield, 2011,p. 2)

Figure 3: Poverty Rate Over 50 Years 
EXAMINING TEACHERS' PARADIGM AND PEDAGOGY IN MANIFESTING CRITICAL HOPE IN LOW-INCOME MIDDLE SCHOOL

This timeline demonstrates that the monumental efforts poured into areas and people of poverty are not transformative solutions, but rather affirmative solutions without the capacity to attack the root causes of poverty at its core (Fraser, 1997). After 50 years of effort towards striking the "War on Poverty," 46 million people still live in poverty across America. America has spent 22 trillion dollars fighting poverty (Rector \& Sheffield, 2011) without significant gain. Pouring money into poverty over time through social programs such as welfare, medical, and unemployment benefits are not transformative solutions and have failed to make a difference over time, and suggests that we are only looking at the macro system to determine the causes of poverty. We need to consider a transformative solution for poverty, and I suggest that we need to consider looking deeper into the micro system in order to break the cycle of poverty in the United States (North, 2008).

This study aimed to critically uncover the continual existence of intersecting inequities visible below the surface in education, which are a direct result of what I have coined to be poverty blindness. Poverty blindness represents a systemic blindness towards the causes of poverty internally and externally representing educational policy, teacher pedagogy, and internalized oppression in the students, relating to the possibility of a broader spectrum. It is taken from the notion of culture blindness, a psychological term that is defined as the incapacity to comprehend how individuals in a different culture experience specific situations due to the systematic construction and alignment of viewpoints, outlooks, and morals of one's own society or culture (N., Pam, 2013). This means that the dominant culture within a society, and the structures of that culture creates continual processes that emphasize the morals, views, and outlooks of that dominant culture. Similarly, the notion of colorblindness stated that people do not see color, only people, even though discrimination shows its face within society in many 
EXAMINING TEACHERS' PARADIGM AND PEDAGOGY IN MANIFESTING CRITICAL HOPE IN LOW-INCOME MIDDLE SCHOOL

forms (Bonilla-Silva, 2017). Looking critically at societal structures that continues oppressive practices for marginalized people of color to benefit the dominant culture creates an awareness of systematic structures, which oppressively provide barriers for marginalized groups. By coining the term to incorporate a broader lens of all people living in poverty and the effects of the blindness from educators, policy makers, and students, poverty blindness suggests the possibility of broader social learning processes becoming institutionalized (Murphy \& Fleming, 2010); it critically examines the (macro) systematic structures within education, and the interactions of inequities in society that are produced in (micro) children of poverty (North, 2008).

\section{Who Are the People Living in Poverty?}

The purpose of the War on Poverty was to provide opportunities for the poor that would generate income for society as whole, instead of relying on and taking money from the government. "The goal of the War on poverty was making taxpayers out of tax eaters" (Rector \& Sheffield, 2011, p. 15). The reality is that those who are born in poverty face systematic structures that make it extremely hard for them to move up in social class because opportunity is linked strongly to social class (Owen \& Sawhill, 2013). As previously stated, even after the Brown v. Board of Education (1954) ruling on racial segregation in schools, there still exists a large segregation in school demographics today, which largely affects their educational opportunities (Ullucci \& Howard, 2015). U. S. Census Bureau revealed that the highest level of poverty is with minorities and people of color with Blacks at 22\%, Hispanics at $19.4 \%$, and Whites at $8.8 \%$ (Fortenot, Semega, \& Kollar, 2018). The same correlation can be made when upon examination of who holds the majority of wealth in the US (see Figure 4). 


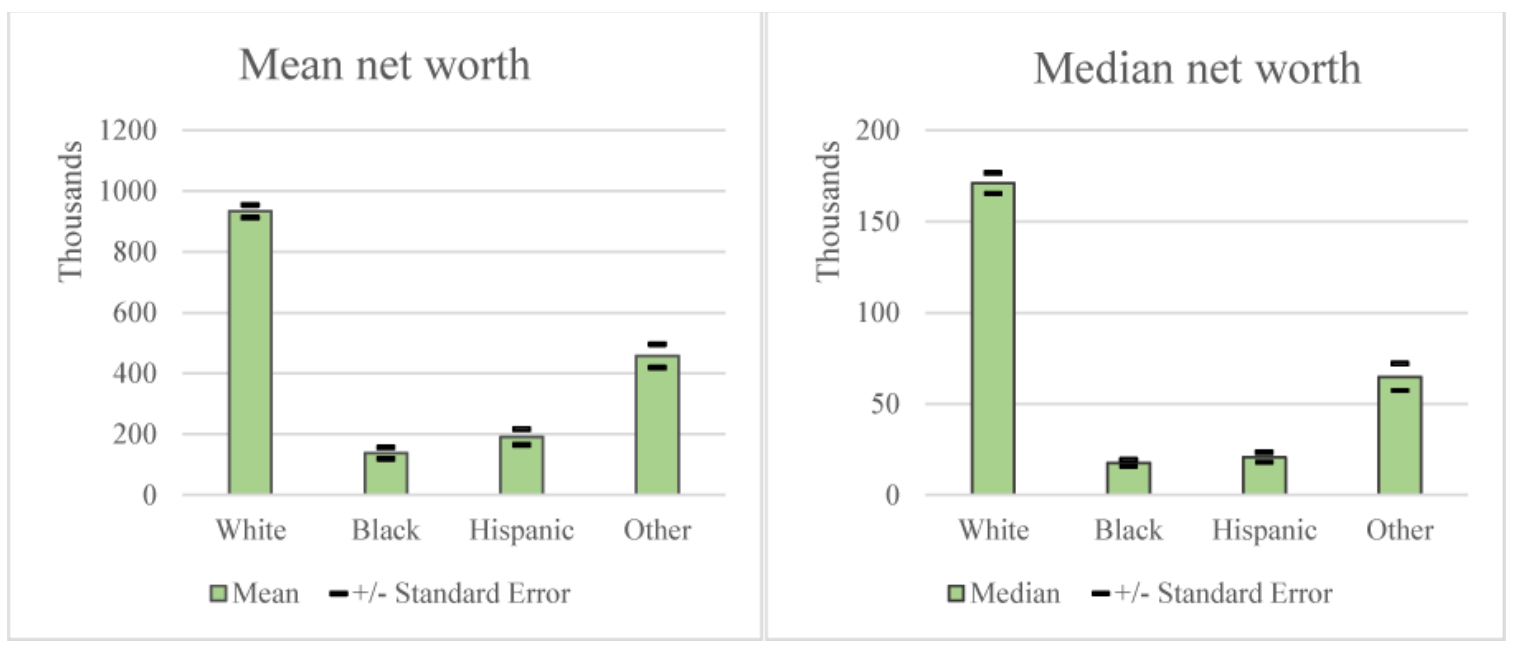

Source: (Dettling, Hsu, Jacobs, Moore, \& Thompson, 2017, Figure 1)

Figure 4: Mean and Median Net Worth

Again, Figure 4 verifies that Blacks have the lowest wealth, with Hispanics having the second lowest wealth, and Whites are holding the majority of the country's wealth.

Consequently, wealth gets passed onto the wealthy, and the lack of capital gets passed onto the poor. These inequities are continual and are enforced by the belief that anyone can succeed in this country if they pull themselves up by their bootstraps (Ullucci \& Howard, 2015). However, when poverty for children equals a less cared for education, lack of opportunities, and lessor amounts of hope for their future, the notion of pulling yourself up out of poverty by your bootstrap is clearly an understatement made in lack of equitable concern or social justice.

\section{How is Poverty Measured in Schools?}

Poverty in schools is measured differently than the poverty thresholds determined by the Census Bureau. One way to calculate poverty in school attendees is by determining how many students qualify for the free and reduced lunch program (Misra, 2015). In a report conducted by Misra (2015), it was shown that degrees of poverty naturally occur dividing the north and the 
EXAMINING TEACHERS' PARADIGM AND PEDAGOGY IN MANIFESTING CRITICAL HOPE IN LOW-INCOME MIDDLE SCHOOL

south of the United States with the exceptions of Utah, Montana, and South Dakota. A minimal amount of poverty is located in the northeastern sections. In California, pockets of poverty are situated mainly in the middle of the state consisting primarily of Hispanics as the largest group and African-Americans as the second largest group (Flint, 2018). As a result, children in poverty in California are also primarily children of color.

\section{How Does the US Public Education System Receive Funding?}

Federal, state, and local agencies determine funding for US public schools. At the national level, vested interest in education comprises a supplemented effort of around $10 \%$ of funding through a variety of programs and laws, while the remaining $90 \%$ rests within state and local funding (Spellings, 2005). The Elementary and Secondary Education Act (ESEA) of 1965 was used specifically for strengthening education through library resources, textbooks, instructional resources, professional development, and grants for children of low-income families (Spellings, 2005). In 2001, the reauthorization of ESEA manifested itself through the No Child Left Behind Act (NCLB, 2002), which was an additional effort at the national level to close the national achievement gap through accountability, options for parents, highly qualified teachers, and research-based instruction (Spellings, 2005).

Title 1, the largest Federal K-12 program under ESEA/NCLB, provides financial assistance to local educational agencies to improve academic achievement to children in high poverty schools (Spellings, 2005). Title 1 requires that states must assess achievement levels of students, provide a report to the parents and the public to determine where the achievement gap is, and designate the funds to meet the identified needs (Neuberger \& Riddle, 2015). The achievement gap between classes in American schools continues to be alarming, as in some areas of the United States, Whites and Asians are separated from all other people of color by up to 40 
EXAMINING TEACHERS' PARADIGM AND PEDAGOGY IN MANIFESTING CRITICAL HOPE IN LOW-INCOME MIDDLE SCHOOL

points (Howard, 2010). Currently in California, the achievement gap between low-income students and affluent students is 32 points as measured by Smarter Balanced Assessment Consortium (Flint, 2018). The achievement gap refers to the difference in knowledge and skill determined by the standardized tests (Howard, 2010).

However, under the U. S. Constitution the responsibility of education primarily rests under state and local funding (Spelling, 2005). In most states, the remaining $90 \%$ of funding is divided in less than half by state and local funding, and a small percent falls under other sources, consisting of private donors, or the Lottery. The local funding varies from district to district because funds are determined by local property taxes (Spelling, 2005). The more affluent the area, the more property tax revenues, resulting in higher funded schools.

\section{Inequitable Access through Inequitable Funding}

Unfortunately, children of poverty also face inequities pertinent to access through funding as there is a tightly bound connection between political economy and the quality of education a student receives (Anyon, 2011). Growing up in a low-socioeconomic area may also mean going to an underfunded school (Misra, 2015). This is a disadvantage because funding determines access related to resources, teacher quality, and opportunities afforded, inevitably leading to inequitable educational experiences (Ladson-Billings \& Tate, 1995; Misra, 2015). Primarily, being underfunded affects teacher capacity, as higher funded school districts are able to attract and keep teachers with more experience and higher levels of education shown to lead to higher student achievement (Darling-Hammond \& Post, 2000; Elliott, 1998; Ferguson, 1991; Ferguson \& Ladd, 1996). Thus, inequitably, more experienced teachers teach the children of privilege and the least experienced are teaching children of poverty (Lalas, 2007; Ullucci \& Howard, 2015). 
EXAMINING TEACHERS' PARADIGM AND PEDAGOGY IN MANIFESTING CRITICAL HOPE IN LOW-INCOME MIDDLE SCHOOL

Out of 50 US states, California remains at the bottom pertaining to educational funding. "Today despite having the world's fifth-largest economy, and the highest GDP of any state, California ranks 41st in per-student funding, 45th percentage of taxable revenue spent on education, 45th in student teacher ratio, and 48th in the number of staff per student" (Flint, 2018, p. 32). California has large areas of poverty, the largest number of English learners in the country (Flint, 2018) while consistently hovering at the bottom of the country as it pertains to educational funding and staff allocated to meet the needs of all diverse learners.

As previously noted, California hovers towards the bottom, 41st in educational spending nationally (Flint, 2018). Furthermore, the low-achievement scores from the standardized tests as measured by the Smarter Balanced Assessment Consortium (SBAC) reflect the investment made in education, as California continues to perform poorly, ranking 42nd nationally (Crave, 2018; Flint, 2018). "California students perform almost a full-grade level below their non affluent peers nationwide" (Flint, 2018, p. 27). The amount of funding invested into students in California is reflected in their achievement scores, as California is consistently in the bottom 10 in spending across the nation, and also in the bottom 10 in results. The education achievement score is based upon the data from the National Report Card, which uses standardized test scores to measure academic achievement. According to Education Week's ranking, "California received a ' $\mathrm{C}$-' overall, notching ' $\mathrm{D}+$ ' grades in $\mathrm{K}-12$ achievement and school finance" (Crave, 2018). Furthermore, since 1944, the prison budget in California has been greater than the budget for all the universities put together (Bourdieu, 1998; Giroux, 2011) inequitably reflecting the investment in education (Flint, 2018) compared to the investment in prison cells. 
EXAMINING TEACHERS' PARADIGM AND PEDAGOGY IN MANIFESTING CRITICAL HOPE IN LOW-INCOME MIDDLE SCHOOL

\section{Inequitable Academic Success}

Subsequently, by examining poverty levels and the educational funding received, we can determine that the states with the higher levels of poverty also had the lowest educational funding, and inevitably, lower academic achievement. For example, Mississippi contains high areas of poverty with the lowest educational funding. As a result, Mississippi came dead last in academic achievement ranked 50th (Education Week Research Center, 2018). The same correlation can be made with Louisiana, ranked at 48th, Alabama ranked at 45th, and as previously noted California ranked at 42nd in academic achievement (Education Week Research Center, 2018; Flint, 2018). Conclusively, nationwide the reality is we are not meeting the educational needs of our children in poverty. In fact, there is no country in the world that achieves well academically pertaining to children of poverty (Cochran-Smith et al., 2016). Inequitably, children of poverty are held to the same standards pertaining to the standardized tests as affluent students, without the same funding and classroom experiences. Therefore, there continues to be a significant difference in academic achievement between students in affluent areas and children in poverty (Ullucci \& Howard, 2015).

\section{Does Funding Really Matter?}

In 1966, in response to the Civil Rights Act (1964), James Coleman and colleagues released a massive study that was commissioned by the National Center of Education Statistics describing the relationship between funding in education and academic achievement called Equality of Educational Opportunity (Coleman, 1966). The report discussed equity issues, and is mostly known for the third section, which identified determinants of achievement. In the conclusion, it discussed that the highest determinant for student achievement was related to students' family backgrounds and their peers, and that quality of school and level of funding 
yielded minimal, or no impact. Then in 1972, economist Kenneth Boulding also noted through his research that higher amounts of educational funding seemed to not generate greater student achievement, which led to many other models for studying the effect of education funding and academic achievement. For example, in 1989 and again in 1996, economist Eric Hanushek declared repeatedly that level of funding is not related to achievement in the real world of public education, which was a welcomed conclusion proposed as fact by the population who is opposed to educational funding redistribution and reform proposals.

However, there is also evidence that funding does lead to higher academic achievement. Educators and the public population who desire to address inequities in education welcome the conclusions that higher funding leads to greater academic achievement. Unfortunately, statistics can be determined through the lens that one frames their research. For example, Rob Greenwald, Larry Hedges, and Richard Laine (1996) determined that many studies by economists have reported an increased academic achievement; if there is a combining of their findings through statistical aggregation, which suggests a significant positive effect due to higher funding due to the generation of resources, higher qualified teachers, and smaller classroom sizes (Greenwald et al., 1996; Hedges \& Greenwald, 1996; Hedges, Laine, \& Greenwald, 1994). This demonstrates that those motivated to change inequities in education state that there are positive, significant results based upon funding, and those who oppose funding reform often find ways to negate it. However, when you look critically at the quality of education given to students in affluent areas, compared to areas of poverty, one can conclude that there are vast inequalities within the equality of education received by students of poverty. This leads to the discussion of pedagogy. 
EXAMINING TEACHERS' PARADIGM AND PEDAGOGY IN MANIFESTING CRITICAL HOPE IN LOW-INCOME MIDDLE SCHOOL

\section{Inequitable Pedagogy: Not All Schools Are Equal (Anyon, 1980)}

In theory, education is the great social equalizer; however, when one critically looks at the educational system below the surface area, education is not the social leveler that it has been believed to be (Anyon, 2011). Theorists argued that poverty explains the achievement disparities (Ullucci \& Howard, 2015) and that the social class of one's parent is in reality the great equalizer determining the quality of education a child receives (Anyon, 2011). "It may be shocking, however, to learn how vast the differences in schools are - not so much in resources as in teaching methods and philosophies of education" (Anyon, 1980, p. 1). Jean Anyon's (1980) article on hidden curriculum explains how all schools are not equal as she discussed the differences in education between four schools she identifies as the Working Class, Middle Class, Affluent Professional School, and Executive Elite School. According to Anyon, there is a difference in instruction, expectations, and student tasks within the social classes leading to the preparation of the society in which they are found. For example, the working class was taught through steps of procedure, and their answers were viewed as right or wrong based on if the steps were followed, or not followed. Anyon's argument was that this pedagogy is intentionally preparing the students for the workforce in the society that they reside. As she went through and did investigations, Anyon saw that the choices the students were given, the instruction that was delivered, and the student work that was expected increased in rigor, relating to the working class found within that societal structure. Anyon looked at the schools found within different levels of social classes with a critical theory lens, and discovered that "All Schools are not equal" (Anyon, 1980, p. 1). This means that there are different levels of pedagogy found within different societies. By examining schools with a critical lens, she was able to discover inequities found within schools of differing levels of social economy. 
EXAMINING TEACHERS' PARADIGM AND PEDAGOGY IN MANIFESTING CRITICAL HOPE IN LOW-INCOME MIDDLE SCHOOL

\section{What Does the Surfaced Macro Data Tell Us about the US Poverty Rate by State?}

As of September 2017, the Official US poverty rate was at $12.7 \%$. States with higher levels of poverty are above the official poverty rate of $12.7 \%$. Table 1 displays the 10 highest states of poverty in chronological order on one column, and the 10 lowest states in K-12 achievement for 2017, in the other. The achievement score is based upon the data from the National Report Card, which uses the High State Testing (HST) scores as a means of measuring academic achievement. Although states move up and down in bottom chronological order for both poverty and achievement, they usually hover somewhere in these areas and rankings from year to year.

Table 1 easily identifies the states with highest levels of poverty and where they fall nationally using the HST to measure academic achievement. When comparing poverty at the national level to the National level HST, even on the surface level, one can determine that areas with high poverty are also the areas with the lowest academic achievement. Therefore, there continues to be inequities in education including funding and pedagogy, and we must consider a critical approach if we want to generate lasting change within these communities.

First, by taking a careful approach on examining poverty levels and the educational funding received, a determination can be made that the states with the higher levels of poverty had the lowest academic achievement. Mississippi was a high poverty state with lowest educational funding. Here we see that it came in last place in academic achievement. The same can be said of Louisiana, at 48th place and Alabama at 45 th. In fact, there is not a state with high funding and low poverty on this list. They all share one common theme: Poverty. 
Table 1

Highest Poverty Rate versus Lowest Academic Achievement

\begin{tabular}{|c|c|}
\hline $\begin{array}{l}\text { States with Highest Poverty Rate } \\
\text { (Hansen, 2018) }\end{array}$ & $\begin{array}{l}\text { States with Lowest K-12 Achievement } \\
\text { (Education Week Research Center, 2018) }\end{array}$ \\
\hline 1. Mississippi $20.8 \%$ & 50. Mississippi $60 \%$ \\
\hline 2. Louisiana $20.2 \%$ & 49. New Mexico 61.8\% \\
\hline 3. New Mexico $19.8 \%$ & 48. Louisiana $62.8 \%$ \\
\hline 4. Kentucky $18.5 \%$ & 47. West Virginia $62.8 \%$ \\
\hline 5. West Virginia $17.9 \%$ & 46. South Carolina $64.4 \%$ \\
\hline 6. Arkansas 17.2\% & 45. Alabama $64.6 \%$ \\
\hline 7. Alabama $17.1 \%$ & 44. Alaska $65.6 \%$ \\
\hline 8. Arizona $16.2 \%$ & 43. Arkansas $66 \%$ \\
\hline 9. Oklahoma $16.3 \%$ & 42. Kansas $66 \%$ \\
\hline 10. Georgia $13.1 \%$ & 41. Oklahoma $66.1 \%$ \\
\hline
\end{tabular}

Source: Education Week Research Center (2018, pp. 2-27)

\section{Inequitable Assessment}

Does poverty affect students' academic performance? Assessments can be viewed as a narrative that tells the journey involving the learning that has taken place (Shulman, 2007). The outcomes displayed the amount of learning, or the amount of effectiveness within the instruction. When the numbers are analyzed with transparency and the data disaggregated, the story is told of what was learned, what was taught, and how effective the instruction was for the students who embodied the lesson. This is a reflective evolutional method to evaluation, or the happy-ever- 
EXAMINING TEACHERS' PARADIGM AND PEDAGOGY IN MANIFESTING CRITICAL HOPE IN LOW-INCOME MIDDLE SCHOOL

after ending to the assessment narrative. However, assessment is not always used in this methodological way.

Education and assessment go hand-in-hand; meaning, whenever there is learning taking place, a measurement of that learning usually follows. In education, the three terms of evaluation, assessment, and testing are synonymous and are often used interchangeably. For this dissertation, evaluation, assessment, and testing are defined as a tool of measurement, which is used to interpret data that has been gathered systematically as a determination of a subject's merit, and effective practice of instruction, by using criteria governed through a set of standards. Within this definition, assessment is important because it shows what students know and what instruction is effective. In the same way, assessment also shows what students do not know and what needs to be taught. If assessment is done effectively, it acts as a tool to inform instruction as to what specifically is in need of targeted instruction, or what needs to be retaught in a different manner, in order for students to reach mastery of the standards, which are being assessed. Standard is a term that also embodies many objectives in education such as the Common Core Standards, the State Standards, the individual goals of the student, the learning objectives, and the mission of the institution. "Assessment focuses on important issues that are central to the mission of higher education-student learning and development (Pike, 2002, p. 131)." This means that assessment also measures the student learning pertaining to the mission and the learning objectives of the institution. Therefore, standard is also a term which embodies many aspects in education.

In terms of equity, an assessment seems to favor the students who belong to the dominant culture, who have the benefits and privileges that encompasses their social class. In addition, assessment data can be dehumanizing for those who do not receive those benefits and yet, are 
EXAMINING TEACHERS' PARADIGM AND PEDAGOGY IN MANIFESTING CRITICAL HOPE IN LOW-INCOME MIDDLE SCHOOL

subjected to taking the same inequitable assessment producing inequitable outcomes (Howard, 2010; Sensoy \& D’Angelo, 2012).

\section{Why Does Evidence Matter in Education?}

In education, assessment is used as a form of accountability with consequences, or rewards based off of the learning outcomes, or the growth that has been shown. The accountability system was put in place during President Bush's No Child Left Behind Act of 2001 (NCLB, 2002) that reauthorized the Elementary and Secondary Education Act, which included Title 1 provisions for disadvantaged students through accountability done with High Stakes Testing (HST), and has been continually enforced through President Obama's Every Student Succeeds Act (ESSA, 2015).

ESSA requires that a state's accountability system must be based on its adopted academic standards. Each state must establish long-term goals that include interim measurements of progress toward those goals for all students, as well as for certain subgroups of students-i.e., economically disadvantaged students, students from major racial ethnic groups, children with disabilities, and English language learners, (Darling-Hammond et al., 2016, p. 5)

These interim assessments are high stakes assessments (HST) that are done annually to measure the growth of the students of the institution. "Under such policies the results of examinations are used to determine students advancement, and to provide criteria for rewarding high-performing schools, or sanctioning those who test scores falter" (Ryan \& Weinstein, 2009, p. 225)." They are used to provide Title 1 funding to schools through the schools Local Control Funding Formula (LCFF), which is the schools accountability report. The purpose of the LCFF is to bring transparency and clarity to the stakeholders involving each school district pertaining 
to the funding has been provided and where it is being dispersed to meet the needs of the students and the subgroups that are in most need based off of the HST.

In terms of equity, an assessment seems to favor the students who belong to the dominant culture, who have the benefits and privileges that encompass their social class. In addition, assessment data can be dehumanizing for those who do not receive those benefits and yet, are subjected to taking the same inequitable assessment producing inequitable outcomes (Sensoy \& D’Angelo, 2012; Howard, 2010).

\section{High Stakes Testing as a Form of Oppression}

There are many negative outcomes that may arise with high stakes testing when it is used as the sole and primary measure of growth. According to Ryan and Weinstein (2009), "Results of HST reforms indicate a plethora of unintended negative consequences, leading some to suggest that HST corrupts educational practices in schools (p. 224).” Since so much is vested into high stakes testing, there can be negative consequences that ultimately affect the students learning. Some of these negative consequences are teaching to the test, falsifying testing results and dropout rates, loss of student engagement, controlling approach compared to autonomous approach, and no feedback. HST intention was to hold teachers and educational institutions accountable to student learning. Unfortunately, research demonstrated HST is providing more negative consequences than positive rewards to students in Lower Social Economic Areas, or the disadvantage in which both NCLB (2002) and ESSA (2015) was intended to impact. Following is a discussion of these negative consequences of HST.

\section{Teaching to the Test}

Teaching to the test refers to a realignment of instruction that focuses what is on the test, instead of a well-rounded quality of instruction focusing on all material. Teachers go through the 
test to determine what will be measured and then refocus the content instruction onto the material that is being tested, leaving out the rest. The results of this are irrelevant, boring, testing drills that prepare students to excel on tests, but produce no real, long-term results of learning that will be beneficial to the students' future (Ryan \& Weinstein, 2009). This superficial form of instruction leaves high performing students unmotivated, bored, unchallenged, and levitated of growth, while low-performing students feel demoralized (Ryan \& Weinstein, 2009). These strategies are noted to be most prominent in low-performing schools usually in low-social economic areas, leaving the gifted checked out and the low performing unmotivated to learn. Thus, the achievement gap in low-performing schools continues to grow.

\section{Falsification of Testing Results and Student Dropout Rates}

The pressure to succeed on high stakes testing provides opportunities for both teachers and administrators to falsify information on score reports and cheating in all levels of the system. For example, students who are known to be categorized as low performing are placed in programs such as G.E.D, occupational certificates, or simply passed onto other schools in the district as to not "drag down their scores (Ryan \& Weinstein, 2009, p. 228)." This results in decreased student achievement and motivation, which ultimately leads to a higher dropout rate for students in these lower-scoring categories. Policies have also been put in place to counter the attrition rates that have been caused by HST. However, research shows the dropout rates have also been falsified, leading to discrepancies in both testing scores and dropout rates (Ryan \& Weinstein, 2009).

\section{Loss of Student Engagement}

Student engagement is an indicator to student learning. However, HST does not focus on student engagement. It is a one-size-fits-all assessment that does not take into account individual 
student needs, students' funds of knowledge (Macias \& Lalas, 2014), or responsiveness to pedagogy. Responsiveness refers to the teacher's ability to determine what students know, what they need to know, and how to make the content culturally relevant by incorporating visuals, strategies, and text that is meaningful to the students which are ways to increase student engagement. HST is not concerned with pluralism or responsive pedagogy. It is uniform, irrelevant, and removes the notion of individualized instruction to meet student needs. When teachers are realigning instruction to conform to the patterns on the HST, they are also removing individualized, relevant pedagogy that low-social economic students with high language barriers are in dire need of.

\section{Controlling Approach Compared to Autonomous Approach}

Controlling approach refers to pressure that is applied to produce an outcome through rewards or consequences, or an attempt to control a certain behavior (Ryan \& Weinstein, 2009). For example, HSTs are related to some sort of rewards or consequences. This is what makes it a high stakes test. There will be a result coming from the test, whether it is a final grade, determines the classes for next year, or placement in a program. Students are aware that it is high stakes, which provides an enormous amount of pressure and stress, which can lead to underperformance during testing. HSTs can also be perceived as too challenging, which leads to a feeling of helplessness and the lack of student motivation to even try. In controlling approach, the focus is on the outcomes of the HST.

On the other hand, autonomous approach is when testing is used to inform instruction. This alleviates the pressure students' feel, increasing the value in the test and the purpose for it. When the test is used to inform, it is individualized, being used as a tool to see what the students' needs are, providing teacher feedback with the goal of student growth. Autonomous approach is 
EXAMINING TEACHERS' PARADIGM AND PEDAGOGY IN MANIFESTING CRITICAL HOPE IN LOW-INCOME MIDDLE SCHOOL

a mind shift, where the focus is on supporting and enhancing student learning, increasing student engagement, and the individualized responsiveness to pedagogy.

\section{No Immediate Feedback}

HST does not offer opportunities to provide students with immediate feedback. Students take these tests and do not get results until weeks or months later, and the results are usually extremely vague. For example, the Smarter Balance Assessment Consortium (SBAC) provides a proficiency level for English and math based on the student's overall scores, which are Standard Exceeded (4), Standard Met (3), Standard Nearly Met (2), or Standard Not Met (1) (California Department of Education, 2016). These scores do not provide the student with feedback that would be considered valuable because they do not know what they did well in and what they need to work on. All they know is if they met the standard or if they did not meet it. They are not told what standard this is referring to as there are many standards in both English and math. In addition, if they did not meet the standard, as is the case for many students in low-socio economic areas, the score serves to diminish their motivation for next year, as they continue to perceive themselves as a failures, not able to be successful academically, or the test was too challenging.

On the contrary, research showed assessments that provide specific, immediate feedback show a significantly larger increase in performance (Opitz, Ferdinand, \& Mecklinger, 2011). This is because students learn what they did right, and how to improve what they are not doing well. They are then intrinsically motivated to perform because they see the value in testing (Ryan \& Weinstein, 2009). Instead, of the assessment being yet, another aspect that they fail in, assessments are used to inform their performance, which increases both engagement and motivation (Ryan \& Weinstein, 2009). 
EXAMINING TEACHERS' PARADIGM AND PEDAGOGY IN MANIFESTING CRITICAL HOPE IN LOW-INCOME MIDDLE SCHOOL

In conclusion, HST contains many negative elements, which can be viewed as a form of oppression for students in poverty. They do not offer opportunities for immediate feedback, which leads to growth. They provide opportunities to falsify testing results and lead to higher dropout rates. In addition, HST encourages teachers to stray from content by teaching to the test and decrease autonomous instruction. Instead, the controlling approach is used, which is negative, stressful, and decreases intrinsic motivation and student engagement. Finally, HST is used as the accountability tool to measure academic achievement and teacher effectiveness. As you can see, HST is an important piece in education because it holds teachers and institutions accountable and is used to provide educational funding. On the other hand, HST can also lead to inequities because of the built-in pressures of accountability, which take the place and importance of educating and meeting the needs of the whole child. When we look critically at HST, we can see that we should be questioning these measures to see if they are continuing the social stratification in education and continuing poverty blindness.

\section{Achievement Gap}

Education is one of America's most cherished assets, providing hopes and dreams for all who live here (Howard, 2010). Education separates the people who have, meaning financially, compared to the people who do not have, meaning poverty. However, in the land of opportunity there exists this continual separation of social classes and equality in education. "Numerous scholars have produced revealing portrayals and penetrating analysis of the manner in which indigenous populations, enslaved Africans, people of Latin and Asian descent, women and poor Whites have been denied educational access" (Howard, 2010, p. 9). The achievement gap refers to the difference in knowledge and skill determined by the High Stakes Tests, standardized testing, and SAT Scores. The achievement gap is concerning because it will ultimately and 
EXAMINING TEACHERS' PARADIGM AND PEDAGOGY IN MANIFESTING CRITICAL HOPE IN LOW-INCOME MIDDLE SCHOOL

negatively impact the future of students determining what future opportunities students have such as the colleges students attend and what classes they are offered. Since education is considered to be the social change agent (Anyon, 2005), the achievement gap ultimately affects the individual student's future because of the opportunities that education provides.

According to Flint (2018), there are many reasons why we have achievement gaps in American schools. The reality is that we cannot discuss the achievement gap without a discussion of race. As previously noted, Whites and Asians are separated from all other people of color up to 40 points (Howard, 2010). Unfortunately, racism still exists in our society today. We have come a long way, and we still have a long way to go. The reality is when any student attends a school which lacks high-quality teachers, high-quality curriculum, and funding for students' achievement, a gap in education will rise. According to Howard (2010),

A close look at educational opportunities during the 1920's and 1930's reveals that African American and Latino students attended underfunded, segregated schools, and in fact, were denied access to better funded institutions for no other reason than their racial and ethnic classification. (p. 28).

One reason why this occurred was because people believed that these students did not have the ability to perform as well as White students. This belief is also an example of modern-day oppression that also manifests in a gap of opportunities provided by and through education.

\section{Recognition and Redistribution}

The educational inequities children of poverty face may be producing the continual achievement gap in American public schools and requires the need for recognition of their collective identities, and redistribution of resources to meet their unique authentic needs (Fraser, 1997; Freire, 1970). Perhaps, there are many citizens within poverty and outside of poverty that 
EXAMINING TEACHERS' PARADIGM AND PEDAGOGY IN MANIFESTING CRITICAL HOPE IN LOW-INCOME MIDDLE SCHOOL

are simply unaware of the inequitable funding in the US education. Since public schools serve their local communities and about half of the funding comes through the wealth within that community, it is important that the representatives of poor communities understand and recognize (Fraser, 1997) the problems that children of these communities' face. The reality is that poor children face additional challenges because their allocated options are to naturally attend poorly funded schools (Biddle \& Berliner, 2002). These schools are not equal to the schools in affluent areas (Anyon, 1980). This means that the representatives of those communities need to understand the needs of the community and how to properly distribute those funds to serve their unique student population. Sadly, often peers of their community underrepresent these populations, especially at the state department level (Biddle \& Berliner, 2002).

In the same way, wealthier communities have highly-funded schools. The successes that are achieved in those communities are culturally passed down through the community members that stay there. This tradition provides these affluent areas with more opportunities through the education they receive and the social capital within the community (Bourdieu, 1986). In addition, since it is widely understood that local communities provide for their school districts, people in these communities do not feel the obligation to pay higher taxes or redistribute their resources and wealth to poorer communities (Fraser, 1997). However, when we have a discussion that pertains to equity stemming from data, the areas that show inequities require a reorganization of the gap, and the need to redistribute funds and or resources to close the gap.

\section{Social Stratification}

Social stratification was analyzed through Karl Marx who built upon Max Weber's analysis of capitalism and ideology (Sensoy \& DiAngelo, 2012). Marxism views capitalism as a 
primary source of systemic inequality between social classes and educational attainment because he believed that the capitalist economy could not function without inequality (Anyon, 2011). Social stratification refers to the concept that, "Social groups are relationally positioned and ranked into hierarchy of unequal value. This ranking is used to justify unequal resources among groups" (Sensoy \& DiAngelo, 2012, p. 4). As critical theorists began to gather and critically examine the world, they sought to eliminate structures that devalue one group, or the minorities (Sensoy \& DiAngelo, 2012) to the benefit of the dominant group.

\section{Privilege as a Form of Power and Systemic}

We all live within a society that consists of individuals who are a part of social structures and systems. These social systems set norms and hierarchies of power within society through institutions such as colleges, churches, communities, and families. Inside these institutions people are shaped through norms that dictate what is appropriate, acceptable, and values, and the inverse of what is inappropriate, unacceptable, and not valued within society. Thus, hierarchies of power are formed in relation to what society has set through norms, values, acceptance, and appropriateness.

Privilege naturally becomes a part of social systems because in order for one to have power there must be those who do not have power. In order for one to be accepted, there must be one who is not accepted, and in order for one to be valued, there must be one who is not valued. According to Johnson (2007), the trouble with privilege is that, "it is embedded in structures of power and inequality that shape almost every aspect of life in this society, from economics to politics to religion to schools and the family" (p. 67). The reality is that privilege for someone in society equals oppression for another in the same society. 
Figure 5 demonstrated how social systems influence individuals and individuals reinforce the social system. "Social life then works through the relationship between individuals and social systems and cannot be understood by looking at individuals alone" (Johnson, 2007, p. 82). Thus, privilege becomes a form of oppression that is normalized in society in such a way that individuals cannot come to realization that social oppression exists on their own because they are constantly shaped by the socializing ideologies that they participate in (Johnson, 2007).

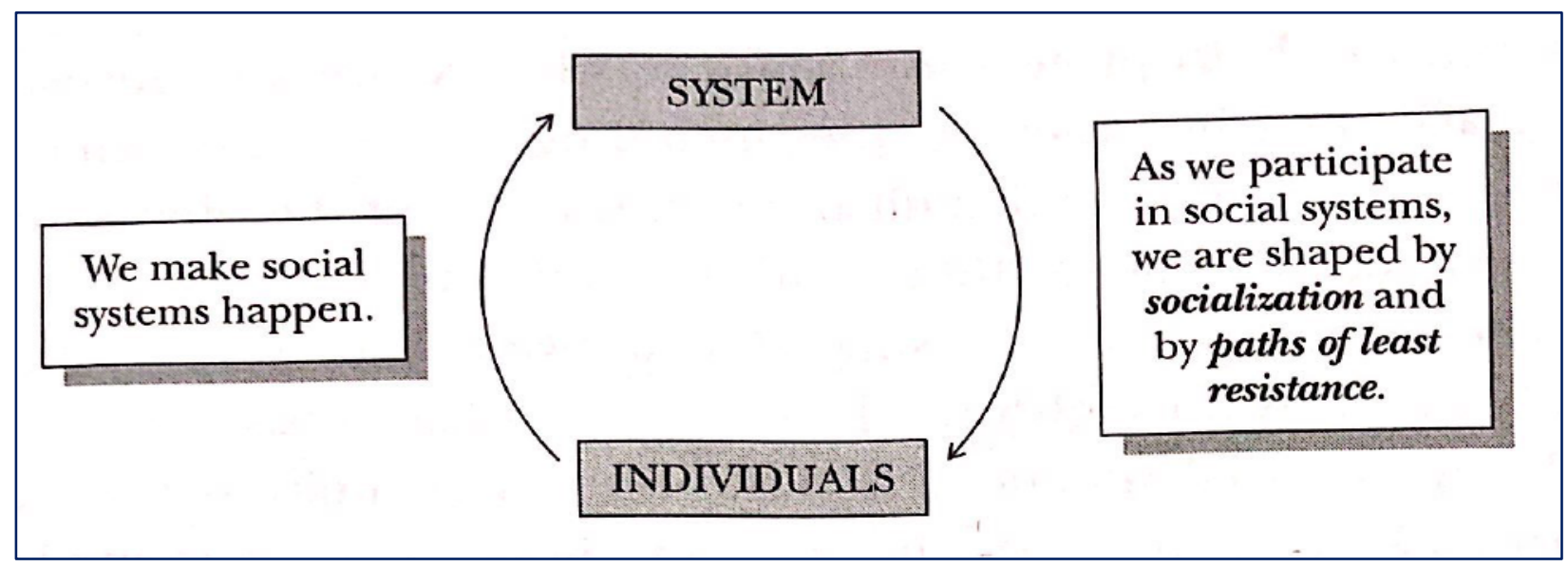

Source: (Johnson, 2007, Figure 6)

Figure 5: System versus Individual

\section{Critical Traditions of Educational Practices}

Critical Theory (CT) emerged in the 20th century from a group of scholars in Frankfurt, Germany to offer an examination and critique of how society could change for the social betterment through examinations of who holds power within societal structures (Sensoy \& DiAngelo, 2012). The term critical in this stance refers to "an intellectual skill of analysis"critical thinking"- as well as to the body of scholarship" (Sensoy \& DiAngelo, 2012, p. 1). Thinking critically means to go below the surface, and consider multiple dimensions visible from many perspectives. The Critical Theory process is complex, which emphasizes a scholarly 
EXAMINING TEACHERS' PARADIGM AND PEDAGOGY IN MANIFESTING CRITICAL HOPE IN LOW-INCOME MIDDLE SCHOOL

approach with the purpose of understanding the historical, political, and social movements behind ideology in order to create change in society (Sensoy \& DiAngelo, 2012).

Critical theory is an examination within power strictures in society and has five distinct characteristics. The first characteristic is that Critical Theory is grounded in a form of political analysis (Brookfield, 2005). This means that a critical lens is employed in order to make a judgment and to examine the inequities found within the relationship of social classes and race in society. Second, Critical Theory is concerned with providing people with the knowledge they need to free themselves from the inequities or oppression they are living in. Third, Critical Theory separates objects and subjects, as they support the theory that represents their emotions on how they desire and dream to live. The fourth characteristic of Critical Theory is that it believes that society can be better through critical analysis leading to change that makes society more equitable. Lastly, Critical Theory desires change, and the transformation acts as proof that the theory was either true or false (Brookfield, 2005).

\section{Critical Pedagogy}

On the same critical view of education, critical pedagogy $(\mathrm{CP})$ evolved out of radical emancipatory efforts derived from democratic principles with the purpose of engaging educators critically with the way societal concerns intersect within education (Darder, 2009). Henry Giroux's (1983) Theory and Resistance on Education is noted with being the first to use the term Critical Pedagogy, as his writings link practices in education to democratic practices in order to transform society for those who have been marginalized and oppressed (Darder, 2009).

Similarly, Paolo Freire's (1970) Pedagogy of the Oppressed critically analyzed power structures as methods of oppression providing a connection between educational practices and society. According to Darder (2009) "Through his views of emancipatory education, Freire 
EXAMINING TEACHERS' PARADIGM AND PEDAGOGY IN MANIFESTING CRITICAL HOPE IN LOW-INCOME MIDDLE SCHOOL

made central pedagogical questions related to social agency, voice, and democratic participationquestions that strongly inform the recurrent philosophical expressions of critical pedagogical writings even today" (p. 5). Thus, Freire strongly influenced educational philosophy through critical pedagogical practices that reinforce Frankfurt's School Focus on Critical Theory. Freire's Critical Pedagogy is a process that leads teachers and students to be social agents of change through an examination of the word and the world, leading toward the process of critical praxis (Freire \& Macedo, 1987). Critical praxis is a cycle of awareness involving five steps including identifying a problem, analysis of the problem, creating a plan for action, implementation of the plan, reflection upon the action, which leads to more action (Duncan-Andrade \& Morrell, 2008). Through problem posing, Freire's emancipatory education methods lead to a critical consciousness, as educators and students question political struggles, exploitation, and the link to educational practices that continues to oppress humans, which for Freire is a dehumanizing state (Darder, 2009). As a result, Critical Pedagogy challenges power structures in society in order to bring forth liberation and humanization through critical consciousness, dialogue, and true generosity as acts of love. Thus, Freire's liberating educational practices sought to create change for those who face inequality as they have been unjustly marginalized in society by social class.

Critical theorists have sought to conceptualize oppression in various ways. Nancy Fraser (1997) discussed the need to recognize individuals and redistribute wealth in order to create equity within society. Bourdieu (1986) discussed how different forms of capital manifest themselves and are intertwined within society to produce economic capital. Freire (1994) discussed oppression and the various ways that it manifested in a capitalist society, and the need for pedagogy of hope to combat oppression. Critical Race Theorists believed that society is stratified to produce continuous benefits for the dominant group at the expense of the minoritized 
group (Ladson-Billings \& Tate, 1995). The Cultural deficit theory is an oppressive ideology that claims that students fail in school because of internal, cultural, or external deficiencies which limit cognitive, linguistic, and motivational abilities for learning. Jean Anyon (1980) discussed the differences in pedagogy in social classes, which in turn reflects the quality of education a child receives. According to Rawlinson (2011), the scourge of poverty critically analyzes the effects of poverty on the child's psychological belief in their capabilities and how that is demonstrated in the classroom. Finally, the Hope Theory (Edwards et al., 2007) discussed how a belief in oneself produces a positive motivation that creates pathways and agency for students and adults to generate success. In the same way, the lack of hope creates a cycle of failure, lack of motivation, and lack of achievement. Therefore, each of these theories addresses modern day oppression in its own lens with a common theme that oppression is socially stratified and leads to inequalities for children of poverty.

\section{Bourdieu's Political Economy}

Bourdieu's (1986) political economy of symbolic power introduced relations between symbolic and material dimensions of social life. Bourdieu (1991) built upon social economic structures introduced by Althusser's (1970) Marxism and dove deeper into the symbolic dimensions of political economy. In this economy, he separated himself from the view of Marxism through three distinct cultural practices of symbolic power-symbolic interests, capital power, and symbolic violence. These three cultural practices have autonomy, but they also intersect and overlap each other within the social realm.

\section{Symbolic Interests}


EXAMINING TEACHERS' PARADIGM AND PEDAGOGY IN MANIFESTING CRITICAL HOPE IN LOW-INCOME MIDDLE SCHOOL

Symbolic interests are the notion of how economic interests relate or translate into noneconomic interests (Swartz, 1997, p. 66). In this way, Bourdieu (1986) separated himself from Marxism by claiming that there are both material and nonmaterial interests that translate into forms of capital. This is a form of Weber's Sociology of Religion where he stated that, "all action is interested" (Swartz, 1997, p. 66). This means that there is a motive behind every social action that is done, which leads to some form of symbolic profit. In addition, "Symbolic interest and material interest are viewed as two objective forms of interest" (Swartz, 1997, p. 67). This demonstrated that symbolic interests and material interest are two separate forms of interests , which are caused by an action. Thus, one could conclude that every action stems from a motive that one has leading to an interest.

In general, Bourdieu (1986) stressed that actions shape individual future responses through what has occurred in their past. A person's past shapes their actions through their culture, traditions, habits, politics, and social responses. These past actions produce future responses. Bourdieu distanced his work from Weber's by stating that "all action leads to interests instead of a means end, or a physical goal" (Swartz, 1997, p. 69). These interests can be both unconscious and conscious actions that are motivated by self. In this way, actions are selfsatisfying, self-vested, and self-interested (Swartz, 1997). Bourdieu's notion of interest brings the connection between economics in materialism and the symbolic economy of cultural and social capitalism.

\section{Power of Capital}

Power of capital refers to the notion that "there are resources of capital that function as a form of social power" (Swartz, 1997, p. 73). Capital of power refers to both social capital in the form of relationships or networks, and the cultural capital in the form of education. Both of 
these forms can translate into sources of income through the workforce (Swartz, 1997). This notion turns into a theory of value where the product, or labor, derives from forms social capital and cultural capital. Bourdieu (1986) separated power into four categories: economic, social, cultural, and symbolic. Economic power includes the structures of material economy meaning money and property. Cultural capital is the education, culture, and traditions found in a person's life. Social capital refers to relationships and networks a person has solidified in their life. Symbolic capital refers to the legitimization one has in their social role through the eyes of others.

\section{Unavoidable Inequalities}

Since three of the forms of power are not quantifiable, meaning there is not a numerical value that can be placed on how much one has or how to evenly distribute it, there is an unavoidable inequality that derives from the unequal distribution among individuals in the forms of cultural, social, and symbolic power. According to Swartz (1997) "These forms of power, and their unequal distribution among individuals and groups explain for Bourdieu why random and perfect competition models are inadequate for understanding social life" (p. 74). Therefore, these forms of power transcend into inequalities found among classes and races within each other, separating the haves with the have nots. In this way, capital is only a form of power if it is invested or transferred from one person to the next because it brings a return in the amount of education one attains. As you can see, these forms of capital transfer into forms of power, which separates classes and races within and amongst each other.

\section{Cultural Capital's Three Dimensions}


EXAMINING TEACHERS' PARADIGM AND PEDAGOGY IN MANIFESTING CRITICAL HOPE IN LOW-INCOME MIDDLE SCHOOL

Bourdieu (1986) analyzed cultural capital in three dimensions. First, embodied capital means internalized cultural goods that one may obtain because they understand the meaning behind it. This begins in childhood as parents invest their time by transferring through communication, teaching, and explaining the cultural goods to their children. Next, objectified capitals are "specialized abilities one has with its culture such as art, music, scientific instruments, that require training or cultural abilities to use" (Swartz, 1997, p. 76). Finally, there is institutionalized capital, or the educational system, which plays a large role in social status and class structure in the job market. For example, parents who "understand the structure that the conversion of economics is derived through the cultural capital investment into their child's education will ultimately lead to the profit of attaining a good paying job" (Swartz, 1997, p. 76). Consequently, the conversion of cultural capital may lead to economic capital.

Therefore, an inequality is found in the historical trend of the affluent transferring and distributing their forms of capital, and it continues to produce social stratification between classes and within races. "In modern differentiated societies, access to sources of income in the labor market depends upon cultural capital in the form of educational credentials and social capital in the form of networks" (Swartz, 1997, p.74). As equity has been discussed and analyzed, ones' access to cultural or social capital can also be considered an inequality.

First, there is no way to regulate cultural or social capital. It is culturally or relationship based. Students can come from a culturally strong background, while other students may not know whom their parents are. Secondly, there are advantages that come with students who have strong distribution of social capital because it can be a type of currency that is used to bring advantages to those students who have not had this type of capital, compared to those who do not; the haves and the have nots (Howard, 2010). For example, students who have strong 
relationships with peers, counselors, teachers, or administrators have support and access to educational opportunities or job opportunities. This is seen as capital because they can get knowledge about programs that are offered, or letters of recommendations from these relationships. In addition, as people get to know people, jobs may be attained based off of a relationship acquired, or social capital, instead of attainment through the skills or knowledge of the individual. This creates inevitable inequities within people who have forms of social capital compared to those who do not.

\section{Symbolic Power}

Bourdieu's (1986) political economy of symbolic power introduced relations between symbolic and material dimensions of social life and presents the notion that social class embodies more than income. Symbolic interests are the notion of how economic interests relate or translate into non-economic interests (Swartz, 1997). Bourdieu's notion of interest brings the connection between economics in materialism and the symbolic economy of cultural and social capitalism.

Power of capital refers to the notion that there are resources of capital that function as a form of social power (Swartz, 1997). Capital of power refers to both social capital in the form of relationships or networks, and the cultural capital in the form of education. Both of these forms can translate into resources of income through the workforce (Swartz, 1997). Symbolic capital refers to the legitimization one has in their social role through the eyes of others. Applied to the plight of children of poverty, if the available resources and attention are not provided to them, symbolically it can be argued that unfairness, inequality, and even "symbolic violence" are accorded to them. Bourdieu (1989 as cited in Swartz, 1997, ) "believe[d] that even in advanced societies the principal modes of domination has shifted from overt coercion and the threat of 
EXAMINING TEACHERS' PARADIGM AND PEDAGOGY IN MANIFESTING CRITICAL HOPE IN LOW-INCOME MIDDLE SCHOOL

physical violence to forms of symbolic manipulation" (p. 82). Symbolic violence according to Bourdieu is the "capacity to impose the means for comprehending and adapting to the social world by representing economic and political power in disguised, taken for granted forms" (Swartz, 1997, p. 89). In this definition, Bourdieu demonstrated that the dominant accepts and continues their dominance and legitimized power by eliciting consent from both those groups they dominate and from themselves as the dominators (Swartz, 1997). Thus, symbolic violence is a contributor to the continual reproduction of the systemic oppression that marginalized groups experience within society.

\section{Paradigm}

Paradigm refers to systems that shape how people think, act, and believe in a society (Johnson, 2007). Society is made up of individuals and systems that interact and shape one's world view, belief system, values, norms, and behaviors that affect diverse groups found within society. Paradigms can be passed down generationally, reinforced through media and print, and reinforced politically through policies, hierarchies of power, and the education one receives. Thus, paradigm is an important concept to understand when considering transforming inequities, systemic oppression, and poverty.

\section{Individual and Institutionalized Paradigm}

Similar to the way Johnson (2007) showed how privilege in society is shaped by systems and those systems shape individuals who reinforce those systems, I am proposing that paradigms are also shaped and reinforced in society through both the institutions and individuals. Kuhn (1962) referred to this as global and local paradigm. Kuhn (1962) believed that nature and the world were constantly in flux. As the understanding of the word paradigm began to grow, noting that it was generously being used in 21 different ways, he distinguished two uses: one for global 
and one for local. Kuhn began to ask questions about what keeps and binds a group together, and what will threaten a group causing it to divide or fall apart. For Kuhn, the answers were found in terms of paradigm. This is the global sense of the word where he emphasized symbolic generalizations, models, exemplars, commitments, and practices. Thus, the word paradigm has evolved over time and has been used in many forms.

For this dissertation, institutionalized paradigm refers to the social systems within societies that shape one's behavior, values, ideologies, worldview, and norms. Individual paradigm refers' to a personal belief, value, ideology, worldview, and norm which in turn reinforces the institutionalized paradigm, or can act as a change agent through acts of resistance to transform it. According to Johnson (2007), "When you openly change how you participate in a system, you do more than change your behavior; you also change how the system happens" (p. 43). Thus, there is a dynamic relationship between institutionalized paradigm and individual paradigm and changing the world must involve both (Johnson, 2007).

Scientifically, paradigm refers to a set of beliefs, practices, and proven solutions that have been researched, tested, and formed by a group of practitioners (Kuhn, 1962). For example, for many years scientists believed that the earth was flat, or that the sun, planets, and the stars orbited the earth. It was not until Copernicus and Galieo found that there were numerous astronomical anomalies that did not fit the prevailing paradigm through extensive research, that they were able to offer a new paradigm that would replace the old paradigm creating a paradigm shift (Johnson, 2007). In order for one to accept the new paradigm, the old one must be replaced (Johnson, 2007; Kuhn, 1962). Thus scientifically, paradigm refers to a proven and accepted belief system found within an institution made up of individuals. 
EXAMINING TEACHERS' PARADIGM AND PEDAGOGY IN MANIFESTING CRITICAL HOPE IN LOW-INCOME MIDDLE SCHOOL

Henry Giroux (2011) referred to the beliefs of educators as their paradigm, which shapes their pedagogical practices and the knowledge that is distributed in the classroom. Freire (1997) referred to paradigm as "human nature being socially and historically situated ... the trajectory in which we make ourselves conscious is marked by finiteness, by inclusion and by characterization of it as historical beings" (p. 49). Freire's argument on objectivism and subjectivism demonstrated that paradigm is systemic and can only be transformed through development of social, cultural, and historical consciousness. "Just as objective social reality exists not by chance, but as the product of humanization, so it's not transformed by chance. If human kind produces social reality, then transforming that reality is an historical task, a task for humanity" (Freire, 1970, p. 25). Therefore, if society wants to engage in transformation, one must become consciously aware of the inequitable knowledge that is given, the lesser opportunities that are provided, and the dehumanizing practices that occur as people are domesticated by objective forces (Freire, 1970). Thus, the world people find themselves in is often who they become.

Henry Giroux’s use of the word paradigm involved having an historical and political consciousness of how one has been shaped in the world. "The concept of paradigm is important not merely because it guides practitioners work, it also illustrates that paradigms are related to the nexus of social and political values in the larger society" (Giroux, 1997, p. 17). Thus, Giroux added to the meaning of the word paradigm the notion of historical and political consciousness.

Similarly, Bourdieu (2005) argued that societal structures and economics combined intentionally reproduce the world in which people reside. In Bourdieu's (2005) work, The Social Structures of the Economy, Bourdieu quoted Bertrand Russell who stated, "While economics is about how people make choice, sociology is about how they don't have a choice to make" (p. 1). 
Bourdieu used this quote to demonstrate that economic and social conditions within a society universally and continually reproduce the knowledge, income, and social order of a society. He based this from his understanding of habitus, social capital, cultural capital, and symbolic violence as means of power that continually provide inequitable opportunities and education for those who do not inhibit those forms of capital. Bourdieu also exerted that in the United States, the spirit of capitalism has produced the notion of individualism, which serves to continue the American social order (Bourdieu, 2005). Subsequently, Bourdieu used the word paradigm to refer to an ideal or standard that is socially, culturally, and economically situated that can become personalized. Bourdieu demonstrated that inequitably the privileged citizens have knowledge and understanding of the adjusted realities related to the norms within society. Thus, transforming these reproductions in society is impossible when there continues to be economic and informational inequality, and the absence of critically aware citizenry between those who are able to enjoy the fruit of these inequitable transgressions compared to those who are void of these forms of capital.

James Paul Gee (2013) built upon Bourdieu's (1986) social and cultural capital when he referred to paradigm as Discourse (with capital letter D), as the ways of using language, acting, interacting, valuing, dressing, thinking, believing, and feeling . . .as well as ways of interacting with various objects, tools, artifacts, technologies, spaces, and times so as to seek to get recognized as having a specific socially consequential identity. (p.55)

Here paradigm is socially and culturally situated and it embodies a person's beliefs, values, and ways of knowing. 
EXAMINING TEACHERS' PARADIGM AND PEDAGOGY IN MANIFESTING CRITICAL HOPE IN LOW-INCOME MIDDLE SCHOOL

Dewey (1938) discussed how education is built upon knowledge and information derived from the past including development of standards, rules, moral conduct, forming habits of action in conformity of these standards, and that it is the purpose of schools to transmit these paradigms to the new generation. "Teachers are the agents through which knowledge and skills are communicated and rules of conduct enforced" (Dewey, 1938, p. 18). However, Dewey's philosophy in education embodied a different approach and perspective to include experiences in education. His view differed from what he called traditional education, which he believed is the continuum of the gulf between what the adult educator knows and the young students know, where the passive student has knowledge deposited directly into them by the professor who forbids active participation. On the other hand, Dewey (1938) believed as society changes, education must progress and connect educational practices to experiences relating the individual student's present, as well as to the knowledge formulated from the past. Dewey believed that educators need to become intimately aware of the local community's physical, historical, economic, and occupational conditions in order to gain an understanding of what knowledge these experiences have given to students. Dewey (1938) believed that all experience is social involving contact and communication and it is found within this place one desires to grow and continue to learn. As a result, educators can be agents in education, forming habits through experiences in education, and impacting the young as "the most important attitude that can be formed is that of desire to go on learning" (Dewey, 1938, p. 48).

Senge (2012) discussed how fields of knowledge do not exist separate from each other, or from the people who study that knowledge. Senge defined paradigm as one of the most complex living systems made up of often invisible networks and interrelationships., ... the ideology of the nature of knowledge and knowing, the teachers' underlying beliefs and 
EXAMINING TEACHERS' PARADIGM AND PEDAGOGY IN MANIFESTING CRITICAL HOPE IN LOW-INCOME MIDDLE SCHOOL

values of the nature of schooling, and social interactions in the learning environment. (p. 686)

In this definition, paradigm is a living system that changes over time depending on experiences, interactions in education, and ways of knowing and belonging to the individuals that are involved. Unfortunately, educators are mostly unaware of their paradigm and how it has been historically, politically, socially, and culturally constructed and how it is manifested in their pedagogy (Giroux, 2011). For the purpose of this dissertation, paradigm is defined as the living system of knowledge and knowing one's world view; belief system; personal experiences; education; personal values; social intersections; and political, social, cultural, and historical consciousness that shapes an educator's pedagogy and practices. Consequently, this dissertation attempted to determine how teacher's paradigm may be directed by hope, stemming from a belief in their students' abilities, and their future aspirations informing their pedagogical practices.

\section{Culture Deficit Theory}

Each year, millions of low-socioeconomic status (SES) minority students of color compete against affluent White and Asians who attend prestigious schools for college, scholarships, and careers. The High Stakes Testing (HST) that is used to calibrate academic achievement exposes the educational failure that SES students face each day. Many theories have arisen with the purpose of explaining these great disparities between education in SES and students in affluent areas, including the culture deficit theory.

Of the several theories that have been advanced to explicate school failure among the economically disadvantaged minority students, the deficit model has held the strongest currency-spanning well over a century, with roots going back further to the beginning of American colonies of the 1600's. (Valencia, 1997, p. 2) 
The cultural deficit theory claims that students fail in school because of internal, cultural, or external deficiencies, which limits cognitive, linguistic, and motivational abilities for learning.

"In race relations, we have social engineers who think up ways of strengthening the Negro family, rather than methods of eradicating racism” (Ryan, W., 1971, p. 8). Instead of focusing on eliminating racism, cultural deficit theory combines all poor students, which are predominantly African Americans, Mexican, American Indians, and minorities into one category to explain away the educational failure, which has been coined as the achievement gap between students of color and White and Asian students that exists from high stakes testing scores (HST). Therefore, deficit thinking is a cruel form of oppression that is used to explain away educational failure and it has taken on three forms: Deficit Thinking as Genetic Based, Culture of Poverty, and Environmental Deficits.

\section{Deficit Thinking as Genetic Based}

In 1958, the level of intelligence and cognitive ability in African Americans were put into question by Audrey Shuey's book titled The Testing of Negro Intelligence where she argued that due to genetics, African Americans contained and will always contain an intelligence deficit compared to Whites. In her interpretation of the testing that was done, African Americans consistently scored 1 standard deviation lower, or 15 IQ points lower than Whites (Shuey, 1966). Sadly, her findings did not go unchallenged, and were widely accepted amongst researchers of intelligence during that time period. Even though most of her research was subject to interpretation; also, she did not take into consideration the many oppressive environmental factors African American faced (Valencia, 1997). For example, when comparing White northerners to White southerners, it was noted that White southerners scored lower because of environmental factors such as lower education in families and farming as the dominant trade 
EXAMINING TEACHERS' PARADIGM AND PEDAGOGY IN MANIFESTING CRITICAL HOPE IN LOW-INCOME MIDDLE SCHOOL

compared to the education contained by the White families in the north. In the same way, southern African Americans had lesser opportunities for education, and came from families who also were uneducated and mostly illiterate, compared to the educational opportunities the northern African Americans had received. Comparisons of southern African Americans to southern Whites would not have been comprehensible, since they did not receive the same amount of education, or come from families with similar educational backgrounds. Additionally, when comparing northern African Americans to northern Whites, northern African Americans went to school in Harlem and were subject to a lesser of quality of education in the ghetto schools compared to the education of prestigious White schools in the north. These environmental factors would contribute to the disparities of the IQ scores between Whites and Black students for both the north and the south (Valencia, 1997). However, Shuey's final thoughts when averaging the African American scores to the scores of the Whites, reflected the genetic hypothesis that originated in the 1920 s as she stated, "The results, all taken together, inevitably point to the presence of native differences between Negros and Whites as determined by intelligence tests (Shuey, 1966, p. 521)." Her thoughts of genetic inferiority gained in popularity, and continued to infiltrate the minds in educational culture, which provided a basis for continual segregation in public schools (Valencia, 1997).

\section{Culture of Poverty}

"To study culture is to study shared codes of meaning" (Geertz, 1973, p. 79). Culture is traditionally viewed as a collection of values, traditions, and behaviors. When using this lens of culture to analyze the disparities in education between poor marginalized students and their White counterparts, it became widely the notion of a culture of poverty exists and creates an intelligence deficit that is seen on testing scores became widely accepted. "Poor, minority 
students in schools were viewed with a lens of deficiencies, substandard in their socialization practices, language practices, and orientation of their scholastic achievement" (González, Moll, \& Amanti, 2005, p. 34). The culture of poverty claimed that poor students come from households, which are deficient as cognitive sources for learning because they have a culture that does not favor learning (González et al., 2005). Educators who believed in this view of culture from a deficit theory treated their students as if they were unable and unwilling to learn, and did so with the intention to explain away the achievement gap that existed between minority students and their White counterparts (González et al., 2005). This makes it easier for educators to accept the reality of the achievement gap as a deficit in culture, rather than a deficit in teaching, or an educational failure. Simply put, the culture of poverty is a way of blame shifting, instead of taking ownership and responsibility.

\section{Environmental Deficits}

Environmental deficit is the notion the inadequate socialization consequentially produces deficits in intelligence. Some factors are inadequate parents, family style, poor relational skills, and the development of law and order. According to Kohlberg and Turiel (1971 as cited in Valencia, 1997) who studied inadequate socialization and the consequences of deficit, "Only those who have social and economic advantage are able to graduate to Kohlberg's higher level of morality" (p. 34). In addition to minorities, it is noted that Kohlberg also considered women as morally underdeveloped (Valencia, 1997).

\section{Oppressive Societal Practices and Structures}

'If we are truly to 'face up to this American Dream,' we must take full account of this interdependence of domination and freedom, privilege and subordination" (Flax, 1998, p. 2). In general, there are several belief systems about the causes of poverty. One perspective 
EXAMINING TEACHERS' PARADIGM AND PEDAGOGY IN MANIFESTING CRITICAL HOPE IN LOW-INCOME MIDDLE SCHOOL

historically supported in America is the meritocratic notion of individualism, which holds that one's personal success or failure is a result derived from individual effort, rather than social structures (Biddle \& Berliner, 2002). America was founded on the belief that it is the Land of Opportunity, one manifests their destiny, and the power is derived from created opportunity based upon individual effort. For instance, this is why we have such a diverse population representing people from all over the world. People migrate here filled with hopeful endeavors that support these ideals within the Land of Opportunity. However, these belief systems can also lead to negative connotation when one does not think critically about people living in poverty, but rather places blame on impoverished persons for their collected lack of success in life (Biddle \& Berliner, 2002).

\section{Oppression}

Oppression refers to unjust systems, orders, behaviors, and attitudes towards humanity, and can occur in the macro, the micro, internally, or externally. Oppression, which leads to dehumanization, gives rise to violence for both the oppressed and the oppressor. It distorts their thoughts, their behaviors, and changes their destiny from a life of peace, liberty, and morality to a distorted life of anger, fear, power hunger, and loss. Charles Taylor, drew on Hegelian notions to argue that,

Recognition and misrecognition ... can be a form of oppression, imprisoning someone in a false, distorted, reduced mode of being. Beyond simple lack of respect, it can inflict a grievous wound, saddling people with crippling self-hatred. Due recognition is not just a courtesy, but a vital human need. (Fraser, 1997, p. 14)

This quote demonstrates that the way we view people or not view people in society can have a long-lasting, internal impact on them. Recognition simply means to acknowledge 
someone's existence, his or her identity. The way society recognizes a person, adds to their selfidentity and their self-worth. A lack of recognition or a misrepresented recognition can cause internalized oppression, which ultimately leads to a lack of hope in one's abilities. For example, if someone in authority, someone who has power over another person, is constantly denying their capabilities and their worth, the person becomes oppressed and begins to believe the view of the authoritative person. They then must fight these internalized thoughts that hinders them from believing in their capabilities and taking risks in life to pursue goals. The outcome is self-hatred, the "why should I try?" It is the Pygmalion effect, that if you believe you can't achieve then you simply will not.

\section{Dehumanization}

Dehumanization refers to the effects of oppression in humanity, and results in internal feelings of unworthiness from both the oppressed and the oppressors (Freire, 1970). This occurs when humans take unjust actions towards another human that gives rise to distorted notions that the oppressed are seen as less human. Humanity is on a journey of realization of self; what being human means and what it does not mean (Freire, 1970). When the oppressed are treated in ways that deny them of this inalienable right, their humanity is stolen, and that act of stealing their humanity affects both parties in invisible ways. In order to combat against the struggle of dehumanization, the struggle of freedom from the oppression as Freire (1970) discussed, we must first recognize that oppression exists as, "concern for humanization leads at once to the recognition of dehumanization" (Freire, 1994, p. 45). In addition, the recognition of dehumanization must come from society including the people involved, both the oppressed and the oppressors. Therefore, oppression affects both the oppressed and the oppressor by taking 
EXAMINING TEACHERS' PARADIGM AND PEDAGOGY IN MANIFESTING CRITICAL HOPE IN LOW-INCOME MIDDLE SCHOOL

away pieces of their humanities, and it prevents both parties from becoming fully human, living in liberty and morality (Freire, 1970).

\section{Internalized Oppression}

People who are oppressed begin to internally believe that they are unworthy of being fully human. Freire (1970) described this process of internalized oppression, "The oppressed having internalized the image of the oppressor and adopted his guidelines are fearful of freedom. Freedom would require them to eject this image and replace it with autonomy and responsibility" (p. 48). This demonstrates that the oppressed are fearful of life outside of oppression because they would be independent and without the guidelines from their oppressors. They do not believe that they are capable of living without their oppressor, and they believe that they deserve the unjust actions they receive because they have this distorted image in their head and they are not fully human (Freire, 1970). Thus, their struggle is in duality, from within themselves as internalized oppression, and from outside of themselves through the oppressors (Freire, 1970).

\section{Sub Oppressors}

The great mystery is of the sub oppressors, which result as the oppressed become oppressors themselves. Their dehumanization has conditioned and structured their thoughts turning the oppressed into oppressors themselves against people in their own community. This occurs as the oppressed seek to become human through what they have been conditioned to understand through their relations with the oppressors; that power over others is what makes you a man, and the more oppressive you are, the manlier you become. According to Freire (1970), "Their ideal is to be men; but for them, to be men it to be oppressors" (p. 47). This is demonstrated when peasants are promoted as overseers of other peasants. Even though their job code has changed, their situation has not changed, meaning the oppressed and the oppressor 
EXAMINING TEACHERS' PARADIGM AND PEDAGOGY IN MANIFESTING CRITICAL HOPE IN LOW-INCOME MIDDLE SCHOOL

relationship remains the same. They have been given additional authority, which means power, and for the peasant, power is made evident through violence.

Sub oppression is also a result of their duality, where they have an admiration of their oppressor and a desire to be freed from them as well. For the oppressed, the oppressor is a model of manhood (Freire, 1970) as they take on the oppressors' identity admiring and desiring what they have: a sense of control and power. On the other hand, the oppressed are angry with their oppressors for the maltreatment and lack of freedom in their life. As a result, the sub oppressors lash out and attack their community members, as a way of attacking their oppressors and gaining a sense of control and power. This is what Freire (1970) referred to as horizontal violence, when the oppressed strike at their comrades creating waves of crime in their communities. Thus, areas of poverty are also areas of high crime, manifested as a result of the dehumanizing and hopeless experiences the oppressed have endured.

\section{Liberation through Generosity}

The ultimate goal of the oppressed is to become liberated, or free from their oppression, but this cannot happen through becoming oppressors themselves, which is the natural progression, or cycle of the oppressed. Nor can it happen from the oppressors who have pillaged, raped, and stolen their humanity (Freire, 1970). Rather, it must come from the oppressed as they consciously choose and seek restoration of both themselves and the oppressor. "Only the power that springs from the weakness of the oppressed will be sufficiently strong to free both" (Freire, 1970, p. 46). This beautiful statement shows that the oppressed is made strong in their weakness through the forgiveness found within them for their oppressors (Freire, 1970). Freire (1994) titled this action of restoration "generosity," meaning that it is undeserved. The oppressors do not deserve, nor have they earned this act of love, but rather it is a selfless act 
of generosity found within the oppressors themselves. According to Freire (1970), "True generosity consists precisely in fighting to destroy the causes which nourish false charity., ... True generosity lies in striving in becoming humans, which work and working to transform the world" (p. 46). This demonstrates that true generosity is one where the oppressed works to restore the relationships within society by becoming the life that fights against injustice, instead of sustaining it. This takes courage, love, forgiveness, understanding, care, and kindness. It is a denial of what is rightfully due to that person, as if payment was owed for all the harm that was caused. The generosity comes from within; it's internally motivated, as the oppressed does not seek a repayment, or compensation for the wrong that has been charged to their life. Instead, there is a forgiveness of this compensation and acts of love are repaid in the place of acts of death (Freire, 1994).

\section{False Generosity}

"This then is the great humanist and historical task of the oppressed: to liberate themselves from the oppressors as well" (Freire, 1970, p. 46). In order for the generosity to be genuine, it must come from the oppressed. False generosity refers to generosity given from the oppressors; it is false because they do not have enough strength within them to liberate the oppressed (Freire, 1970). Any attempt to soften the oppressor's power is weak because any attempt to take away the power of the oppressor ultimately results in despaired attempts to keep it, which continues the oppression (Freire, 1970). According to Freire (1994), "An unjust social order is the permanent font of this false generosity, which is nourished by death, despair, and poverty" (p. 46). This statement reveals that false generosity continues to produce a society of poverty and despair. 
EXAMINING TEACHERS' PARADIGM AND PEDAGOGY IN MANIFESTING CRITICAL HOPE IN LOW-INCOME MIDDLE SCHOOL

\section{Revolution}

Revolution involves the process of establishing liberation through confronting the problem. It is a critical recognition of the cause (Fraser, 1997; Freire, 1994) and the will to transform the situation with the struggle of becoming fully human (Freire, 1994). This cannot be done through false generosity as that is used to keep the oppressed subdued and in submission (Freire, 1970). False generosity provides tiny strands of hope that keep the oppressed working and moving according to the laws and orders of the oppressors. True generosity brings revolution, as the internal motivation desires independence, liberty, and hope to be free from their oppression (Freire, 1970). Liberation involves the courage to face the oppression and the web that it has entangled inside of both parties. It is important to note that revolution in itself does not remove the shadow of oppression (Freire, 1994). The removal of the conditioned behaviors involves the ability for both the oppressed and the oppressor to face their fears. For the oppressed, they have a fear of freedom because they have become dependent on the oppressor for life. They must conquer this fear and break the bondage that has removed all responsibility and autonomy for one's choices. In addition, they must remove the feeling of admiration for their oppressor and release them from their hands. According to Freire (1994), "Freedom is acquired by conquest, an indispensable condition for the quest of human completion" (p. 48). For these reasons, liberation is an act of critical recognition and reflection, and true transformation comes from critically recognizing the cause and then seeking to change it (Freire, 1994).

Aligned to the principles conceived by Freire (1970), Jonathan Kozol's (2007) The Shame of a Nation examined issues pertaining to social class, inequalities in education, and race as power structures by using stories and experiences of those who have faced the social 
EXAMINING TEACHERS' PARADIGM AND PEDAGOGY IN MANIFESTING CRITICAL HOPE IN LOW-INCOME MIDDLE SCHOOL

consequences related to poverty. As Kozol critically analyzed the connection between power structures and education, he documented that human oppression also included the conditions of lesser education for racialized communities (Darder, 2009). In addition, Peter McLaren's (2003) Life in Schools asserted that Critical Pedagogy creates change through analysis of cultural politics between critical educators and students leading towards dual empowerment. McLaren's (1994) Critical Pedagogy prepares educators to examine the relationship between social and power relations that are found between knowledge, formal and hidden curriculum, and social reproduction. For McLaren (2003), the hidden curriculum is what is taught and tconsists of learning conditions, rules, teaching styles, and grading practices (Duncan-Andrade \& Morrell, 2008). Furthermore, as previously stated, Jean Anyon's (1980) Social Class and the Hidden Curriculum of Work also built upon Freire's (1970) work similarly to McLaren (1994), as she examined the inequitable educational practices and opportunities afforded between social-class, and discovered that all schools are not equal.

\section{Critical Race Theory}

From this critical view of education, Ladson-Billings and Tate (1995) applied this perspective by formulating critical race theory (CRT) in education. Critical Race Theory critically examined the connections between race, laws, and societal structures and how those views are found in education (Ladson-Billings, 2013). Critical Race Theory has five tenets. The first tenet states that racism has been normalized and it is embedded in the policies and practices of society (Ladson-Billings, 2013). This tenet proposes that racism is the normal way of society and the common everyday experience for people of color (Ladson-Billings, 2013). The second tenet of Critical Race Theory is what Derrick Bell (1980) called interest convergence. LadsonBillings (2013) suggested that Derrick Bell was the father of Critical Race Theory as he stated 
that those of privilege would seek racial justice only when they believed that it would benefit them in some way (Ladson-Billings, 2013). Thus, civil rights activists need to align their interests with the interests of the dominant group in order to create change for marginal groups who are oppressed (Ladson-Billings, 2013).

The third tenet of Critical Race Theory asserts race as a social construction. Even though humans are scientifically mostly similar species, hierarchies of power have created social structures on the basis of race as there are significant disparities related to positions of power in society and quality of life (Ladson-Billings, 2013). For example, race as a social idea impacts where someone lives, what schools they will attend, and what career will develop out of those educational opportunities afforded (Ladson-Billings, 2013). Consequently, these disparities are determined by the color of one's skin.

The fourth tenet of Critical Race Theory is intersectionality and identity. As Critical Race Theory developed, it was discovered that race was the result of many hierarchical factors in society that leads towards oppression. The reality is that people represent multiple identities. Intersectionality occurs when a person is oppressed in multiple forms or ways. According to Delgado and Stefancic (2017), "Intersectionality means the examination of race, sex, class, national origin, and sexual orientation and how their combinations play out in various settings" (p. 58). This demonstrates that people are not binary and have multiple social identities and categories of difference. Thus, Critical Race Theory states that because society has many forms of hierarchy a person may be oppressed in more than one way, which affects their identity.

The final tenet of Critical Race Theory presents voice or counter narrative through storytelling. Storytelling in society presents the view of the dominant and the way the world is viewed through the narrator. Similarly, history is told through the view of the dominant culture 
EXAMINING TEACHERS' PARADIGM AND PEDAGOGY IN MANIFESTING CRITICAL HOPE IN LOW-INCOME MIDDLE SCHOOL

for morality preservation and education (Ladson-Billings, 2013). The purpose of counter narrative is to provide the opposing view leading to an understanding of how legal principles operate in society in order to advance larger concerns, and advocate for racial and social justice (Ladson-Billings, 2013). Thus, Critical Race Theory desires to deconstruct laws by providing a lens to view the connection between race and policies and bring enlightenment to racial injustices within the power structures in society.

\section{Culturally Relevant Pedagogy}

From the review of critical traditions of educational practices, culturally relevant pedagogy (CRP) was formed as a remedy inside the classroom for children of poverty (LadsonBillings, 2005). According to Ladson-Billings (2005), the way to break the systemic cycle of inequities for children of poverty is to focus on the kind of education minority students need in order to be successful. Culturally relevant pedagogy was developed by Ladson-Billings (1994) to empower students through Critical Pedagogy that uses culture to engage students intellectually, socially, emotionally, and politically by providing meaningful learning experiences. According to Ladson-Billings (2005), CRP develops a socio-critical consciousness by stressing how teachers conceptualize the culture of self and of their students in order to develop culturally congruent teaching practices (Howard, 2010). Thus, pedagogy that responds to culture and acknowledges an individual effectively provides inclusive and equitable access to education (Harmon, 2012).

Culturally relevant pedagogy is a form of Critical Theory as it critically examines the world through multiple lenses and teaches students to engage in problem-solving skills in order to improve society through actions of social justice (Harmon, 2012). "Ideally, culturally relevant pedagogy can prepare students to change society, not just merely fit into society" (Harmon, 
EXAMINING TEACHERS' PARADIGM AND PEDAGOGY IN MANIFESTING CRITICAL HOPE IN LOW-INCOME MIDDLE SCHOOL

2012, p. 13). In addition, culturally relevant pedagogy supports Critical Race Theory, as education is viewed through the diverse children who experience it. Thus, Culturally Relevant Pedagogy focuses on how teaching practices can effectively improve education for children of color. As a result, Culturally Relevant Pedagogy has shown to be dually beneficial for both students and educators (Harmon, 2012).

In general, pedagogical strategies used by teachers to provide learning experiences that are culturally congruent have taken on many forms of terminology by educational anthropologists such as cultural appropriateness, culturally compatible, culturally responsive, and culturally relevant (Ladson-Billings, 2014). Similarly, Geneva Gay (2000) formulated her own version called culturally relevant teaching (CRT) that is defined as "the cultural characteristics, experiences, and perspectives of ethnically diverse students as conduits for teaching them more effectively" (Gay, 2002, p. 106). In addition, Culturally Relevant Teaching facilitates engagement as culture is used to provide context of students' lived experiences, and applies it to the academic content knowledge they are learning to make it more personal and meaningful, providing background knowledge, or frames of reference to pull upon, in order to make meaningful connections for learning (Gay, 2002). This practice is defined as cultural scaffolding in teaching diverse students by using their own culture and experiences to facilitate engagement expanding their academic knowledge and achievement (Gay, 2002). Thus, there is a place for Culturally Relevant Teaching in order to facilitate engagement in all content areas in low-income schools (Gay, 2002). As a result, academic performance for low-income students have shown to improve as students are equitably taught through their own cultural experience, facilitating engagement through the discovery of personal value in what they are learning (Gay, 2002; Ladson-Billings, 2014). 
After an analysis of some of the critical traditions of educational practices, Table 2 shows the commonalities found are analysis of systemic inequities, inequitable access, and a desire to make changes to diminish oppression for marginalized groups through empowerment and voice. In tenets one and two, the critical traditions of educational practices focus on the social and political analysis of the society and how individuals are inequitably socialized in a variety of contexts. Tenets three and four emphasize the impact and accessibility of the individual cultural identities and intersectionalities in creating changes in the society. Lastly, tenet five emphasizes humanization, voice, and empowerment for democratic participation in order to diminish oppression.

Table 2

Critical Traditions of Educational Practices (CTEP)

\begin{tabular}{|c|c|c|c|c|}
\hline Theory & $\begin{array}{l}\text { Critical } \\
\text { Theory }\end{array}$ & $\begin{array}{l}\text { Critical } \\
\text { Pedagogy }\end{array}$ & $\begin{array}{l}\text { Critical } \\
\text { Race Theory }\end{array}$ & $\begin{array}{l}\text { Culturally } \\
\text { Relevant } \\
\text { Pedagogy }\end{array}$ \\
\hline Purpose & $\begin{array}{l}\text {-Examines } \\
\text { structures of } \\
\text { power }\end{array}$ & $\begin{array}{l}\text {-Analyzes } \\
\text { practices of } \\
\text { schooling to } \\
\text { democratic } \\
\text { principles for } \\
\text { transformation } \\
\text { in society }\end{array}$ & $\begin{array}{l}\text {-Examines } \\
\text { race in } \\
\text { connection to } \\
\text { laws, policy, } \\
\text { and structures } \\
\text { of power. }\end{array}$ & $\begin{array}{l}\text { - The use of } \\
\text { diverse cultural } \\
\text { characteristics, } \\
\text { experiences, and } \\
\text { perspectives as } \\
\text { conduits for } \\
\text { teaching them } \\
\text { more effectively }\end{array}$ \\
\hline Tenet \#1 & $\begin{array}{l}\text {-Political } \\
\text { Analysis }\end{array}$ & $\begin{array}{l}\text {-Critical } \\
\text { analysis of } \\
\text { the world and } \\
\text { the word }\end{array}$ & $\begin{array}{l}\text {-Belief that } \\
\text { racism has been } \\
\text { normalized in } \\
\text { society }\end{array}$ & $\begin{array}{l}\text {-Belief that } \\
\text { systemic } \\
\text { inequities for } \\
\text { children of } \\
\text { poverty can be } \\
\text { broken }\end{array}$ \\
\hline Tenet \#2 & $\begin{array}{l}\text {-Provides } \\
\text { knowledge in }\end{array}$ & $\begin{array}{l}\text {-Develops a } \\
\text { critical }\end{array}$ & $\begin{array}{l}\text {-Interest } \\
\text { convergence }\end{array}$ & $\begin{array}{l}\text {-Develops socio } \\
\text { cultural }\end{array}$ \\
\hline
\end{tabular}


EXAMINING TEACHERS' PARADIGM AND PEDAGOGY IN MANIFESTING CRITICAL HOPE IN LOW-INCOME MIDDLE SCHOOL

\begin{tabular}{lllll}
\hline & $\begin{array}{l}\text { order to be } \\
\text { self-liberated }\end{array}$ & $\begin{array}{l}\text { consciousness } \\
\text { to fight } \\
\text { oppression }\end{array}$ & consciousness \\
\hline Tenet \#3 & $\begin{array}{l}\text {-Separates } \\
\text { objects and } \\
\text { subjects }\end{array}$ & $\begin{array}{l}\text {-Problem } \\
\text { Posing }\end{array}$ & $\begin{array}{l}\text {-Race as a } \\
\text { social } \\
\text { construction }\end{array}$ & $\begin{array}{l}\text {-Holding high } \\
\text { expectations for } \\
\text { all students }\end{array}$ \\
& system & &
\end{tabular}

Table 2 (Cont'd.)

\begin{tabular}{lllll}
\hline & $\begin{array}{l}\text { Critical } \\
\text { Theory }\end{array}$ & $\begin{array}{l}\text { Critical } \\
\text { Pedagogy }\end{array}$ & $\begin{array}{l}\text { Critical } \\
\text { Race Theory }\end{array}$ & $\begin{array}{l}\text { Culturally } \\
\text { Relevant } \\
\text { Pedagogy }\end{array}$ \\
\hline Tenet \#4 & $\begin{array}{l}\text {-Believes } \\
\text { society can be } \\
\text { better }\end{array}$ & $\begin{array}{l}\text {-Critical } \\
\text { Praxis }\end{array}$ & $\begin{array}{l}\text {-Intersectionality } \\
\text { and identity }\end{array}$ & $\begin{array}{l}\text {-Cultural Identity } \\
\text { development }\end{array}$ \\
& $\begin{array}{l}\text {-Change is } \\
\text { proof that the } \\
\text { theory was } \\
\text { true or false }\end{array}$ & $\begin{array}{l}\text {-Humanization } \\
\text { through } \\
\text { generosity, } \\
\text { love, and } \\
\text { dialogue }\end{array}$ & $\begin{array}{l}\text {-Voice or } \\
\text { counter } \\
\text { narrative to } \\
\text { create change }\end{array}$ & $\begin{array}{l}\text {-Empowerment to } \\
\text { make change } \\
\text { through equity, } \\
\text { access, and social } \\
\text { justice }\end{array}$ \\
& & & &
\end{tabular}

Source: Adapted from Brookfield, 2005; Freire, 1970; Ladson-Billings, 1994; \& Ladson-Billings \& Tate, 1995

In addition, the review of critical traditions of educational practices revealed that all these commonalities are all socially situated (Hawkins, 2014) meaning that when applied to examining the children of poverty these students "are particular people with particular interests, points of views, personalities, and experiences" (Cooper, 2014, p. 367). Research showed that there is disparity in achievement when comparing children of poverty to children in affluent areas related to whom they are and how they learn (Lalas et al., 2016; Ullucci \& Howard, 2015). As a result, through the use of these critical traditions of educational practices, the plight of children of poverty can be analyzed and social-justice leaders can be guided in creating changes to address these inequities related to educational access, inclusion, and academic success. 
EXAMINING TEACHERS' PARADIGM AND PEDAGOGY IN MANIFESTING CRITICAL HOPE IN LOW-INCOME MIDDLE SCHOOL

\section{Hope Theory}

Hope is commonly used to define an emotion or a feeling that changes based on one's circumstances. I am defining hope to be an internal belief that changes are possible, driven by a visualized trajectory leading towards the goal (Lalas, Charest, Strikwerda, \& Ordaz, 2019. In this definition, simply having a feeling of hope does not create hope. Hope must be accompanied with the action. When one hopes and does not do anything about their goals it turns into hopelessness and despair. An action-less person, who lives inside the struggle turns into a hopeless person. "Hopelessness and despair are both the consequence and the cause of inaction or immobilism" (Freire, 1994, p. 2). This means that hope must involve action, and the more that one hopes and acts upon their hope, their belief strengthens because of the learned practice and exercise of hope.

The hope theory is a construct that is useful for making both positive and negative correlations in adults and children. Hope is defined as "a positive motivational state that is based on an interactively derived sense of successful agency and pathways" (Edwards et al., 2007, p. 83). Agency is defined as "The motivational component to propel people along their imagined routes to goals., ... also known as willpower, agency reflects the perceived ability to initiate and sustain movement toward a goal" (Edwards et al., 2007, p. 84). This means that there is a cognitive analysis of one's ability to succeed. Agency looks at all the options and considers if success is possible. When barriers arise, agency reflects a determination to meet the desired goal, and depending on one's level of hope, the thoughts are either positive or negative in the form of "I can do this," or "I can't do this" (Edwards et al., 2007). Goals are defined as "targets of mental action sequences" (Edwards et al., 2007, p. 84). 
Goals need to be "sufficiently important" to the individual, again connecting to the notion of motivation through the four principles. When one is processing and analyzing their ability to achieve, there must be at least a middle level of probability that success is possible. "To achieve goals people must perceive that they are capable of imagining one or more routes to their goals" (Edwards et al., 2007, p. 84). This is referred to as pathway thinking, meaning that there is a way that one can visualize towards success. When barriers arise, pathways provide flexibility in determining an alternative route towards success (Edwards et al., 2007). These two components of agency and pathways are learned through experiences in childhood, and influence emotions of success or failure (Edwards et al., 2007). According to the hope theory, "Successful goal pursuits of high-hope individuals cast a positive emotional set over the process in general. Likewise, individuals who lack hope may enter the thought sequence with negative feelings toward goal pursuits" (Edwards et al., 2007, p. 84). Therefore, hope is a learned cognitive process that affects one's motivation and ability in their belief to reach their goals. 


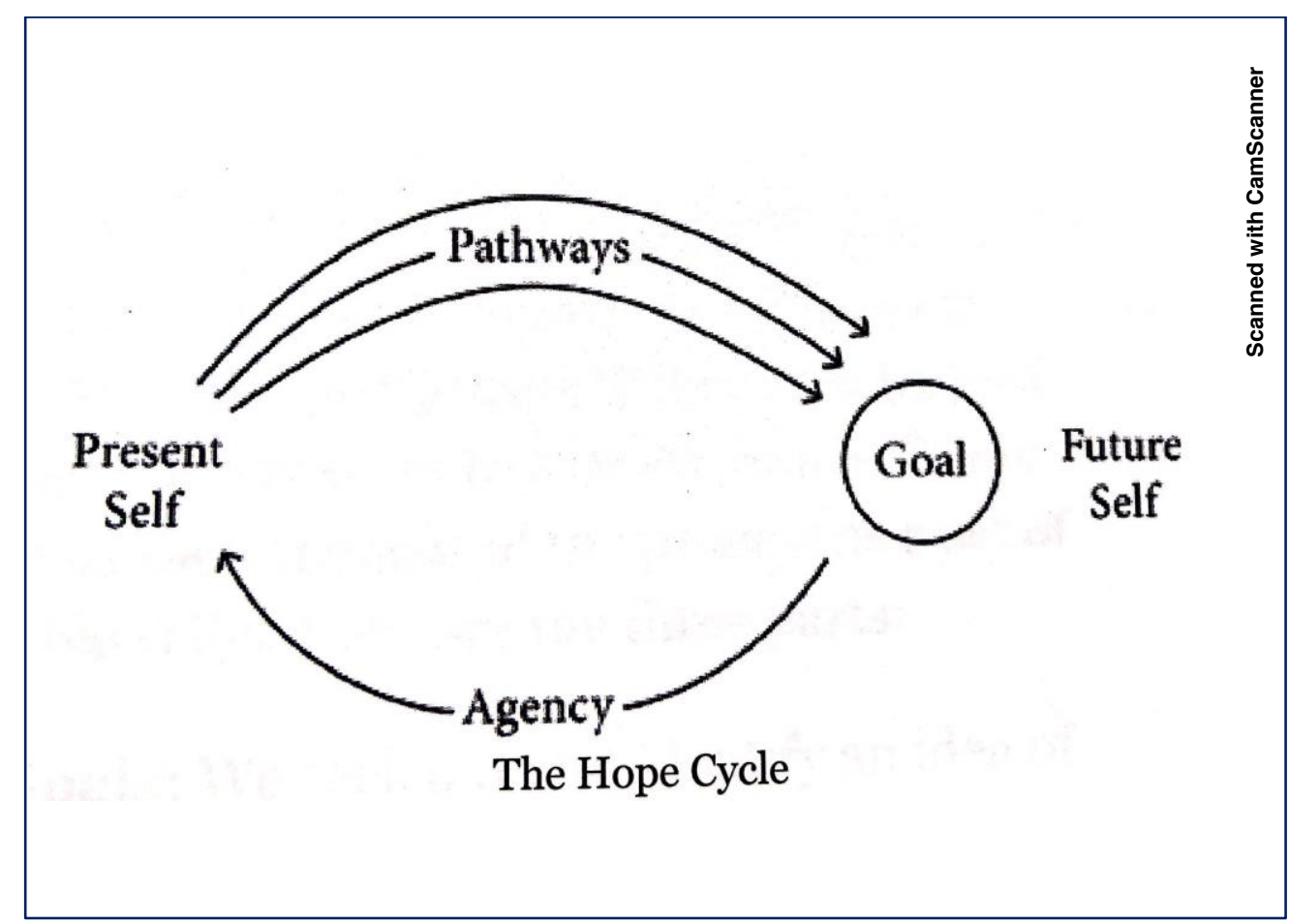

Source: Adapted from Lopez, 2013,p. 25

Figure 6: The Hope Cycle

\section{The Hope Theory and Academic Achievement}

The hope scale is used to measure hope in adults and children. There has been extensive research done to assure validity of the hope theory and the scales used to measure hope (Edwards et al., 2007). Hope scores do not measure intelligence, but rather a correlation between one's hope and achievement (Edwards et al., 2007). Children and adults with higher hope scores have shown to perform better on standardized tests, predicted higher grade point averages, lower dropout rates, and higher graduation rates (Edwards et al., 2007). The correlation is simple: the higher the hope, the higher the achievement. The same can be said with the inverse: the lower the hope, the lower achievement that is based upon their negative feelings toward past pursuits with goals (Edwards et al., 2007). This is because there is an internal, self-perceived competence 
EXAMINING TEACHERS' PARADIGM AND PEDAGOGY IN MANIFESTING CRITICAL HOPE IN LOW-INCOME MIDDLE SCHOOL

level in one's abilities, which determines the amount of effort and resoluteness to stay the course based upon passed successes or failures. Therefore, hope is linked to self-efficacy; it is part of the same construct (Zhou \& Kam, 2016).

\section{The Hope Gap}

\section{Hopelessness}

"Unfortunately, many Americans live on the outskirts of hope-some because of their poverty, and some because of their color, and all too many because of both. Our task is to help replace their despair with opportunity" (Woolley \& Peters, n.d., para. 1). According to Freire (1993), "Hope is an ontological need. Hopelessness is but hope that has lost its bearings, and become a distortion of the ontological need" (p. 2). This means hope is vital to human existence. Without hope in one's future, there is no will to struggle. Children in poverty face daily obstacles that require effort and strength to persevere (Ullucci \& Howard, 2015). Freire (1994) used the term struggle to represent these daily obstacles that are embedded in their life. They are considered oppression including oppressive policies, oppressive teaching practices, and oppressive behaviors. "Without a minimum of hope, we cannot so much as start the struggle" (Freire, 1994, p. 3). In order to even begin the struggle, there must first be hope.

A critical examination of hope leads one to the recognition of hopelessness. An examination of hope and hopelessness is necessary to explain how a lack of hope manifests itself just as strongly as hope, but in a negative manner. "As expected, low hope individuals are more likely to be affected by stressors and become derailed in goal pursuits" (Edwards et al., 2007, p. 85). This means when one has low hope they go through the same analysis of the desired goal and their perceived ability to attain it. Prior negative emotions arise, and distinguish any motivation to pursue their goal. "When we fight as hopeless or despairing persons, our struggle 
will be suicidal" (Freire, 1994, p. 2). This quote means that when a hopeless person fights against inequities without the ability to believe that change is possible, they will become victims of their attempt to struggle, and they will give up in their despair. As individuals progress towards their goal, cognitively, and if there is negative feedback, they will most likely stop pursuing their goal. Freire (1994) stated, "When it becomes a program, hopelessness paralyzes and immobilizes us. When we succumb to fatalism, it becomes impossible to muster the strength we absolutely need for a fierce struggle that will recreate the world" (p. 2). Thus, hopelessness forms and represents a hope gap in the zone of proximity between hope and hopelessness. As hopelessness is formed and continues to increase as goals are not pursued or met. Therefore, I present the notion that a closing of the hope gap is needed before the struggle of closing the achievement gap can begin in children of poverty.

However, high hope individuals receive positive feedback from their positive past experiences which aids them in a continual pursuit towards their goals. According to the hope theory, "As individuals progress toward goal attainment, the success feedback from overcoming the stressor reinforces the individual's hopeful thinking" (Edwards et al., 2007, p. 85). Therefore, there is a continuous cycle as hope breeds achievement, and achievement breeds opportunity, and opportunity breeds more hope. Thus, the continual cycle produces or reduces hope, depending upon the success or failures one personally experiences. I am presenting two cycles of hope: Hopeful and Hopeless (see Figure 7). 


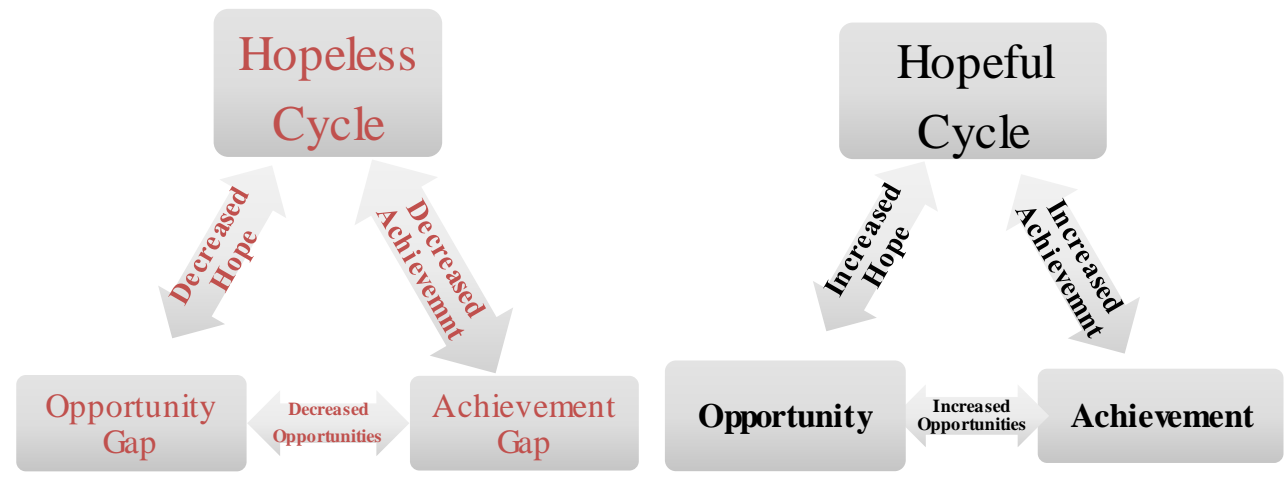

Figure 7: Cycles of the Hopeful and the Hopeless

\section{Hope, Motivation, and Student Engagement}

Research stated that when teachers enact instructional strategies through the four factors of motivation: sense of belonging, autonomy, meaningfulness, and competency that motivation leads to student engagement in the classroom (Turner et al., 2014). Self-determination theory stated that motivation is an internalized drive to meet the psychological needs of relatedness, competence, and autonomy (Deci \& Ryan, 2015). Autonomy develops stronger identifications with the importance of attempting a task. Students who internalize the value in what they are doing are personally connected, and result in higher cognitive learning. Competence means that a student has a belief that they can achieve. Relatedness means that there is a sense of belonging within the classroom environment. The students begin to identify with the learning space that is facilitated by the teacher and the school. Research shows that teachers who support competence, autonomy, and relatedness have higher levels of student engagement and academic success (Niemiec \& Ryan, 2009; Deci \& Ryan, 2015).

Motivation is a universal need. Some psychologists have suggested that the concept of autonomy is only relevant to western culture. However, research done on autonomy in various 
EXAMINING TEACHERS' PARADIGM AND PEDAGOGY IN MANIFESTING CRITICAL HOPE IN LOW-INCOME MIDDLE SCHOOL

cultures shows that regardless of the culture or the values, autonomy is necessary in both eastern and western cultures (Deci \& Ryan, 2015)

Maslow's (1943) hierarchy of needs stated that behavior is motivated by the desire to satisfy needs that are not met (Webb \& Norton, 2009). In Maslow's hierarchy the physiological needs of food, clothing, safety, and shelter are at the bottom, followed by needs pertaining to security, social, esteem, and self-actualization. According to Maslow, "Basic lower level needs serve as potential motivators until they are satisfied, then they are no longer motivators" (Webb \& Norton, 2009, p. 149). This is important because people possess different levels pertaining to their hierarchy of need. This leads to the third level of social needs. As Webb and Norton (2009) stated, "If they do feel secure in their work environment and feel they received adequate compensation, motivators such as belonging and acceptance become important" (p. 149). In general, in order to understand what motivates educators and students on campus an examination and reflection will be needed to determine the levels of motivation.

Many students in high-crime areas face daily attacks on their basic needs of food, clothing, safety, and safety shelter which may result in negative behaviors and disengagement (Rawlinson, 2011). Since teachers have the most influence inside the classroom, it is important for them to understand what motivates and engages their student population. "Some scholars have gone so far to state that teachers are the most valuable influence on students' performance in the classroom" (Howard, 2010, p. 33). High-quality instruction and student performance go hand-in-hand; this means they are interrelated. It is a known fact that students learn by doing. If students are not producing, but rather they are sitting in a chair, filling in bubbles and empty lines on a worksheet, their skills are not going to develop or improve. Effective teachers understand what motivates their students and how they learn, while increasing the rigor, the skill, and the 
EXAMINING TEACHERS' PARADIGM AND PEDAGOGY IN MANIFESTING CRITICAL HOPE IN LOW-INCOME MIDDLE SCHOOL

knowledge simultaneously. "Good teachers bring students into living communion with the subjects they teach" (Senge, 2012, p. 688). Subsequently, effective educators realize that the task is just as important as the instruction and they focus their efforts on student engagement, which leads to student outcomes.

\section{Student Engagement}

Research on student engagement has shown the difference between engagement and motivation. Motivation is the intention or aspiration to do something while engagement pertains to observable, action-oriented behaviors. Skinner and Pitzer (2012) explained their view of "engagement as the outward manifestation of motivation" (p.22). Through effective engagement, students are able to learn making engagement an important indicator of academic success that can predict how a student will perform in school (Reeve, 2012). Student engagement can be different from classroom to classroom and from lesson to lesson. It is important to clearly define what engagement is in order measure it properly. Skinner and Pitzer (2012) defined engagement as "the active verb between the curriculum and actual learning." Reeve (2012) defined engagement as the extent of a student's active involvement in a learning activity. Along with understanding the definition of engagement, it is also important to know what indicators and facilitators of engagement are (Reschly \& Christenson, 2012).

There are many forms of engagement from what can be easily seen to what is happening in a student's mind and heart. Ensuring that all forms of engagement are targeted is important to a well-balanced classroom. Engagement is multidimensional and includes engagement that is observable, engagement that can be seen, academic and behavioral activities, and internal, cognitive, and affective aspects (Reschly \& Christenson, 2012). The following four types of student engagement are considered indicators of engagement. 


\section{Observable}

\section{Academic}

Academic engagement focuses on the academic side of the classroom. How engaged are students throughout the day? Are students participating in classroom conversations? Are they asking and answering questions? How much effort are students putting into their assignments? (Mahatmya et al., 2012). As an educator who works in an urban school district with a large portion of marginalized student groups, academic engagement feels as if it is what educational policy makers traditionally feel is the most important aspect of the educational system, highertest scores. Other examples of academic engagement indicators can be increased reading scores and math scores. These examples can help teachers determine the effectiveness of direct instruction and to determine if a concept needs to be retaught or if the students understood and are ready to move forward (Skinner \& Pitzer, 2012).

\section{Behavioral}

Behavioral engagement focuses on positive and negative behaviors exhibited in the classroom. Examples of behavioral engagement include attendance, staying on class, completing work and disruptive behaviors (Mahatmya et al., 2012). Behavioral engagement is important due to the fact that a student's risk for poor performance can be linked to poor behavior (Mahatmya et al., 2012). Positive behaviors included staying on task and being able to follow instructions. Just like negative behavior can be a predictor of poor academic performance, positive behavior can indicate academic success Mahatmya et al., 2012). Providing an environment where all students have the potential to have positive and successful behavior lies in a teacher's ability to 
EXAMINING TEACHERS' PARADIGM AND PEDAGOGY IN MANIFESTING CRITICAL HOPE IN LOW-INCOME MIDDLE SCHOOL

provide a safe and well-structured classroom, build relationships and trust with their students, and to know who their students are, and what facilitates their engagement (Lalas, et al., 2016; Mahatmya et al., 2012).

\section{Internal}

\section{Cognitive}

Cognitive engagement focuses the student's perception (Reschly \& Christenson, 2012), how they feel and think about school activists, how the socialize with other students, as well as looking at a student's intrinsic motivation, self-efficacy and a positive self-perception (Mahatmya et al., 2012). In addition, cognitive engagement is connected to critical thinking skills. What critical thinking levels of questions are being asked and at what level are students expected to think and perform. When a student builds strong cognitive engagement skills there is a higher chance that as they grow and continue their educational journey, they will maintain higher engagement in their education (Mahatmya et al., 2012) as they have learned and developed skills and knowledge on how to critically think and learn in school and in life. Strong cognitive engagement goes hand in hand with a student's ability to see the value of learning and helps them to set their own personal learning goals (Reschly \& Christenson, 2012).

\section{Affective}

Affective engagement is "a level of emotional response characterized by feelings of involvement in school as worth pursuing" (Finn \& Zimmer, 2012). Affective engagement establishes incentives for students to engage in school activities and to work harder to overcome challenging school activities (Finn \& Zimmer, 2012). Affective engagement is connected to the enjoyment a student feels, excitement, and the internal value one may have related to what is being taught, and the task the student is doing. This particular form of engagement is not easily 
seen or spotted during observations. In order to accurately determine if affective engagement is evident, interviewing students or providing surveys that ask specific questions about how a student values school and their sense of belonging is needed (Finn \& Zimmer, 2012).

\section{Agentic}

Agentic engagement is measured by a student's ability to enrich their own learning experience (Reeve, 2012). Students have the ability to enrich their own learning by being active learners. Instead of sitting quietly and soaking in information, they actively learn by asking higher-level questions and by providing input in what lessons they would like to learn (Reeve, 2012). This is when the students change the atmosphere in the room, when they become agents of their own learning and or the learning of the peers. Agentic engagement is shown in leadership, empowerment, student voice, and action. This can be observed by students that ask questions and are willing to lead their peers.
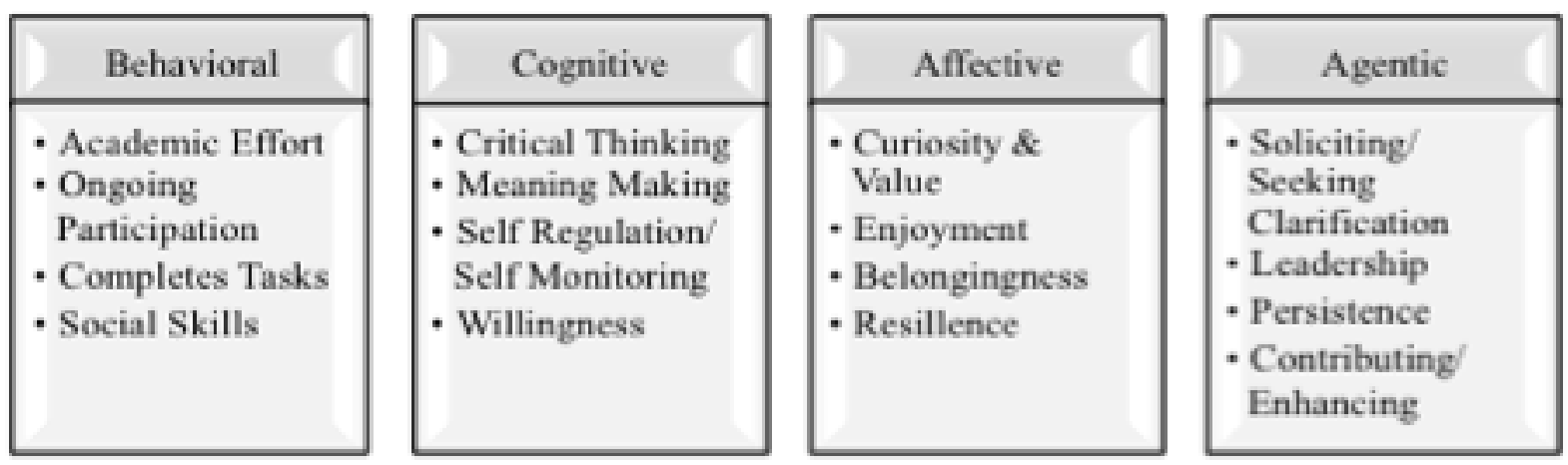

Figure 8: Facilitators of Engagement

Facilitators are "explanatory casual factors, that have the potential to influence the target" (Skinner \& Pitzer, 2012). Teachers are key facilitators that promote engagement. Teachers have the ability to promote a student's intrinsic motivation by establishing high expectations and 
building lessons around topics that connect with their students (Skinner \& Pitzer, 2012). How a teacher structures the classroom can help provide a safe environment where relationships are established and trust is built between the teacher and the students and the student and their peers. This trust can help students to feel safe enough to take risks that they might not otherwise take in a hostile environment. When teachers are able to build relationships, form connections with their students, establish trust, and demonstrate care, research shows that students will want to reach high expectations in order to make the teacher proud (Skinner \& Pitzer, 2012).

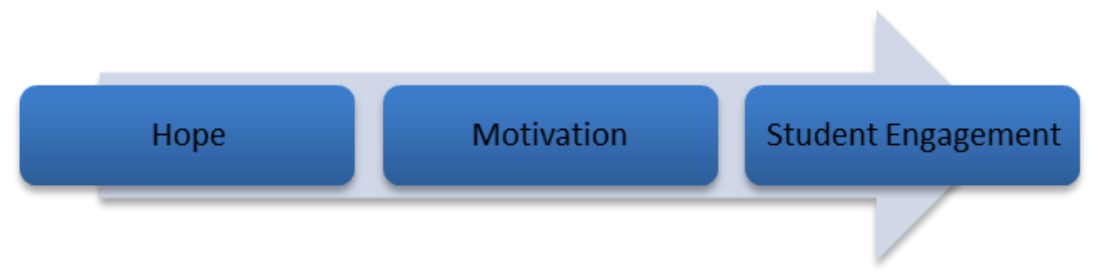

Figure 9: Raising Levels of Hope Produces Change in Motivation and Engagement

\section{How Do We Break the Hope Gap Cycle?}

When we look critically at the entire picture, there is researched evidence to support that underneath the surface there lies this dire need to increase the hope in students of poverty. The High Stakes Testing data that educators are immersed in, that students see and are told over and over again they are failing, they are in the red, and that standards are not met or reading and math scores are below grade level, all leads to deficit thinking and deficit internalized paradigm in both educators and students of poverty. This is complicated by the stories they hear from society that they are poor, and looked down upon because of their social status or even because of their race. In addition, those stories that play over and over in their minds as they struggle with the scourge of poverty that tells them, "I Can't," and goes with them wherever they go (Rawlinson, 2011). These conditions and life factors diminish one's hope in their quest for success, as data 
EXAMINING TEACHERS' PARADIGM AND PEDAGOGY IN MANIFESTING CRITICAL HOPE IN LOW-INCOME MIDDLE SCHOOL

recently reported shows that $70 \%$ of children in poverty will stay in poverty (Mason, 2017).

Subsequently, the detailed literature review on poverty, educational inequities, paradigm and pedagogy, traditions of critical theories as foundational literature, the hope theory as empirical literature, motivation and student engagement, leads to the dire need of examining the manifestations of Critical Hope in teachers paradigm and pedagogy for students in low-income middle schools. As Paulo Freire (1994) states, "We need Critical Hope the way a fish needs unpolluted water" (p. 2).

\section{Narrative Inquiry}

Narrative inquiry is a form of storytelling used in educational research to focus on the study of lived experiences (Conle, 2006). It describes the phenomenon of personal experiences and is rooted in a circular dimensional practice of inquiry and discovery (Conle, 2006). Narrative inquiry is used to study lived experiences through narrative modes of inquiry at its base (Conle, 2006). The aim is to bring understanding and clarity to a particular phenomenon through authentic voices of the participants as they engage in telling of lived experiences. As the stories are told, and recalled from memory, themes emerge through theoretical exploration and interpretation of data sets (Conle, 2006). Narrative inquiry uses concrete language to help facilitate specific factual statements through narrative discourse which may result in a change of phenomenon as the narrative in itself presents opportunity for change for those who are engaged in the discourse (Conle, 2006). This fluidity allows for emerging of conditions and knowledge with the understanding that definitive knowledge is not a possibility (Conle, 2006).

However, by using Habermas's (1984) Theory of Communication Action and Communication Reality, Conle (2006) described that truth claims can be verified through theoretical discourse, "Narrative Inquiry might serve to clarify lived values and to determine 
what the good might be for a particular life or a particular society or culture" (p. 157). This demonstrates that narrative inquiry can serve to clarify lived experiences and be used as a method change for a particular society. Narrative inquiry can be a successful way to bring understanding to teacher educators who serve marginalized and diverse students as their experiences and encounters are told through their voices. In this way, it can be used to shift polarized positions and to bring about positive social encounters through collaborative and mutual construction of sociocultural histories in students of poverty for education (Conle, 2006). 
EXAMINING TEACHERS' PARADIGM AND PEDAGOGY IN MANIFESTING CRITICAL HOPE IN LOW-INCOME MIDDLE SCHOOL

\section{Chapter 3. Methodology \\ Introduction}

The objective of this qualitative study of teachers of low-income students was to examine the manifestations of Critical Hope in teachers' paradigm and pedagogy and to design professional development for teachers on how to acquire pedagogy of "Critical Hope." According to the hope construct, hope is defined as "a positive motivational state that is based on an interactively derived sense of successful agency and pathways" (Edwards et al., 2007, p. 83). The pedagogy of "Critical Hope" that this dissertation is trying to frame and formulate emphasizes the use of the critical traditions of educational practices including optimism, ethics, criticality, social consciousness, caring relationships, sense of community, democratic agency, and humanizing practices in order to elevate students' consciousness of their world and to be hopeful of their successful spaces in relation to that world. Through narrative inquiry, 10 teachers were interviewed and observed to determine: (1) their understanding of hope; (2) how that understanding of hope is manifested in their teaching; and (3) how their teaching practices related to hope influenced student engagement in middle school. The interviews of teachers about hope reflect their view of how hope influences students' success and the impact on lowincome student engagement given their social and cultural environments. The rationale is the need for voices of low-income middle school teachers to be heard, understood, and reported via stories and shared experiences of how their pedagogy (Senge, 2012) and paradigm (Giroux, 2011) either increased or diminished hope for their students' success.

\section{Research Design}

The objective of this qualitative study of teachers of low-income students was to determine how the voices of teachers reflect their perceptions of hope for student engagement 
EXAMINING TEACHERS' PARADIGM AND PEDAGOGY IN MANIFESTING CRITICAL HOPE IN LOW-INCOME MIDDLE SCHOOL

and success in middle school. Conceptually, this research reveals through teachers' voices how their pedagogy and paradigm informs their desire for their students to be hopeful in their quest for success. Theoretically, the research objective necessitated the use of critical theories in education such as Freire's (1974) education for critical consciousness, pedagogy of hope (1994), and pedagogy of the heart (1997), hooks' pedagogy of hope (2003), and other related critical theories (Brookfield, 2005; Murphy \& Fleming, 2010; Freire, 1970; Giroux, 2011; LadsonBillings \& Tate, 1995; Saltman, 2018) and the Hope Theory (Edwards et al., 2007) as the conceptual framework in order to establish a link between pedagogy, paradigm, and hope. The practical and educational value of this research was to provide an opportunity to identify classroom practices that reflect hope as they emerge in the authentic voices of teachers.

In using narrative inquiry, this dissertation sought to bring authentic teachers' voices to the forefront through storytelling, so that they become a part of the educational literature on hope, pedagogy, and paradigm and their connection to student engagement. Specifically, this narrative inquiry sought answers to the following questions: (1) To what extents do the voices of teachers of low-income students reflect Critical Hope in their paradigm and pedagogy? (2) What themes related to teachers' language and behavior of hope, teachers' instructional delivery of hope, and teachers' lesson planning of hope emerge when observing and listening to teachers? (3) What recommendations for educators can be made to reflect the manifestations of "Critical Hope" in teachers' paradigm and pedagogy? The rationale was to give teachers in low-income middle schools the forum to manifest their understanding of Critical Hope in their paradigm and pedagogy. A qualitative methodological approach using narrative inquiry was selected to seek answers to the research questions used to study, understand, and reconstruct the lived 
EXAMINING TEACHERS' PARADIGM AND PEDAGOGY IN MANIFESTING CRITICAL HOPE IN LOW-INCOME MIDDLE SCHOOL

experiences of teachers in the realm of practice while delving into conceptual exploration (Conle, 2001).

These research questions will give voice to teachers of low-income students who are often overshadowed, or left out of the discussion, but who have the lowest student outcomes of all sub groups (Flint, 2018). The rationale was to give teachers in low-income middle schools the forum to manifest their understanding of Critical Hope in their paradigm and pedagogy. A qualitative methodological approach using narrative inquiry was selected to seek answers to the research questions used to study, understand, and reconstruct the lived experiences of teachers in the realm of practice, while delving into conceptual exploration (Conle, 2001). Consequently, this research yields stories related to hope that will inform teachers, administrators, and policy makers on how to work effectively with children of poverty whose voices and experiences have been traditionally and historically silenced, or disregarded in the current system of education.

\section{Narrative Inquiry}

Narrative inquiry as the methodology in this qualitative research design was used to elicit the voices of teachers from a low-income middle school. Low-income students were chosen because they represent the lowest achieving sub group across the nation on standardized tests and college acceptance rates (Flint, 2018). This underperformance can lead to negative visions and hope for their future such as the "school-to-prison pipeline" and high school dropout rates for this population (Giroux, 2011). The age range of middle school was chosen for this study because it represents a pivotal development age of adolescence for low-income students for academic development and hope for their future, as, there is a group of students who complete middle school and chose not to pursue a high school education. Narrative inquiry was used because it allows for an in-depth investigation of the meaning given to experiences of 
EXAMINING TEACHERS' PARADIGM AND PEDAGOGY IN MANIFESTING CRITICAL HOPE IN LOW-INCOME MIDDLE SCHOOL

participating teachers in order to reveal themes embedded in storytelling that may reflect hope and recognize what the needs of low-income students are.

Additionally, narrative inquiry is a form of storytelling used in educational research to focus on the study of experiences through the use of voice (Connelly \& Clandinin, 1990). Voice is used as an outward expression of lived experiences with our social environment (Bakhtin, 1981). Narrative inquiry describes the phenomenon of personal experiences and is rooted in a circular dimensional practice of inquiry and discovery (Conle, 2001). Narrative inquiry is used to study experiences through narrative modes of inquiry at its base (Conle, 2001). The aim of this research was to bring understanding and clarity to a particular phenomenon through the telling of lived experiences (Connelly \& Clandinin, 1990).

As the stories are told, and recalled from memory, themes emerged through theoretical exploration and interpretation of data sets (Conle, 2001). Narrative inquiry uses concrete language to help facilitate specific factual statements through narrative discourse which may result in a change of phenomenon as the narrative in itself presents opportunity for change for those who are engaged in the discourse (Conle, 2001). This fluidity allows for emerging of conditions and knowledge with the understanding that definitive knowledge is not a possibility (Conle, 2001).

Furthermore, Conle (2001) described that truth claims can be verified through theoretical discourse as narrative inquiry may assist with clarifying lived values and to determine what may be good for a particular society or culture. This demonstrates that narrative inquiry can serve to clarify lived experiences and be used as a method for change in a particular society. In this way, research relating to the experiences of teachers of low-income students becomes the phenomenon, or the story and the inquiry with the use of open-ended questions and the 
EXAMINING TEACHERS' PARADIGM AND PEDAGOGY IN MANIFESTING CRITICAL HOPE IN LOW-INCOME MIDDLE SCHOOL

participants' voices used to construct these experiences becomes the method (Connelly \& Clandinin, 1990).

\section{Data Collection Procedure}

This dissertation employed narrative inquiry as a qualitative research technique. The qualitative process involved eight steps: (1) identification of teachers of students in a low-income middle school who are willing to share their experiences; (2) development of the interview questions that would draw out the lived experiences of teachers related to paradigm, pedagogy, and hope; (3) development of the observation tool including the formulation of the elements of Critical Hope as facilitators that influence teachers' paradigm and pedagogy, and its impact on student engagement indicators; (4) actual interviewing of teachers; (5) classroom observation on indictors of student engagement; (6) collection and reading of the transcribed narratives and observations; (7) description and analysis of the content of the teachers' narratives and classroom observations; and (8) reporting of findings and drawing out of conclusions emerging from the description and analysis.

\section{Setting}

Teachers were interviewed in their classrooms or at the campus library. This was based on the preferences of the teachers. Interviews in the classroom happened after the school day was completed, in quiet classrooms that were free from distractions. The school is located in southern California.

\section{Sampling}

The selection of the participants was conducted by using purposive sampling. Purposive sampling is used when the participant sample is chosen based on the qualities of the participants who are proficient and well informed with the specific phenomenon of inquiry (Etikan, Musa, \& 
Alkassim, 2016). Participants were 10 teachers of low-income students in middle school. This is purposive sampling because the individuals who are teachers of low-income students also have the quality of serving in a trauma-sensitive middle school in an urban area with high crime. A trauma-sensitive school undergoes specific professional development related to adverse childhood experiences and how that may impact behavior, social and emotional responses, and academics for these students (Felter \& Ayers, 2016). This trauma-sensitive approach is designed to create an empathetic culture and is shown to improve the relationships between the students and the teachers (Felter \& Ayers, 2016). Thus, these individuals are highly qualified due to their lived experiences. Therefore, a narrative inquiry with purposive sampling was the best research method because of the lived experiences the teachers have with being in a trauma-sensitive school working with low-income middle school students.

\section{Participants}

The Participants are 10 classroom teachers with various levels of experiences and content areas to provide a broad sample from a low-income, trauma-sensitive, middle school.

\section{Positionality}

The Primary Investigator is also a teacher in the same school district that the Participants work in. There is a pre-existing relationship because all the teachers are employed in the school district where the Primary Investigator is a teacher. There is not a hierarchical relationship, which could affect responses as the Primary Investigator is a teacher and the Participants are also teachers. There is a peer-to-peer relationship. The Primary Investigator does not have any authority over the Participants, or any say on pay raises, hiring, or extra duty payment, so there was no coercion. 
EXAMINING TEACHERS' PARADIGM AND PEDAGOGY IN MANIFESTING CRITICAL HOPE IN LOW-INCOME MIDDLE SCHOOL

\section{Gate Keeper}

The Principal Investigator obtained informed consent from all Participants through the University of Redlands Consent to Participate in a Research Study agreement. All Participants read and signed the consent to participate form prior to any interviews.

The informed consent included information regarding the purpose of the study, how data would be collected, the expected time of participation, the possible risks associated with participation, the possible benefits to participation, any compensation, the process of maintaining confidentiality, the fact that participation is voluntary, the fact that Participants may leave the study at any time, and contact information for the researcher. Additionally, Consent to Participate agreements contained contact information for mental health services which Participants can utilize should interviews bring up any unresolved emotional responses related to working with students in low-income schools. A copy of the Consent to Participate in a Research Study form is Figure 12. All Participants were over the age of 18. Therefore, it was not necessary to obtain the consent of a child. All Participants in the study were volunteers. In addition, there were no negative influences used for those not interested in continuing with the study.

\section{Protection of Participants}

Since the Participants in this study are representatives of specific characteristics, a targeted recruitment process was used. The Participants are individuals who are teachers in a trauma-sensitive school with low-income students. The sample was purposive sampling due to the important qualities of the targeted Participants (Etikan et al., 2016). Potential teacher Participants were given a recruitment letter. Teachers who were recruited will be referred to by 
an Alphanumeric to keep their identities private. This process will be beneficial to connect information to the teachers while keeping their identities private and protected.

The primary investigator will destroy all the data collected. The hardcopy will be shredded and the digital data will be destroyed, which will completely eliminate all of the electronic data files. Data will be destroyed after the completion of the dissertation and the related articles are composed or after five years, whichever is longer. In addition, all data will be destroyed using the guidelines that have been established by the University institutional policies and the state laws.

\section{Potential Risks of the Study}

This research project had minimal risk to Participants because no economic, physical, or emotional harm occurred. To ensure teachers would not lose instructional time in classes, teachers attended interviews immediately after school. All interviews were done on a voluntary basis, and teachers were prompted that they did not have to answer any questions that may cause them emotional discomfort. If emotional discomfort was visible and occurred during the interview, the researcher reminded the Participant that they do not have to answer the question, if they do not want to. The researcher explained the use and the purpose of the audio recording before each interview began and ensured the Participant that their information would remain anonymous. In addition, the researcher informed the Participants that all data will be completely destroyed following the completion of the study. Finally, the researcher reminded the Participants that they will meet for a third time to review the transcripts of the first two interviews and to ensure that the reporting is accurate. The email address of the researcher will be provided and the Participants will be reminded that they can contact the researcher at any time with questions and or concerns regarding the study. 
EXAMINING TEACHERS' PARADIGM AND PEDAGOGY IN MANIFESTING CRITICAL HOPE IN LOW-INCOME MIDDLE SCHOOL

The potential for a breach of confidentiality was minimized by using the following: an Alphanumeric to refer to each Participant; storing of information was on a password-protected, secure computer; and completely destroying all documents and related articles of the study at the conclusion of the dissertation or after five years, whichever is longer in congruence with University policies and state laws.

\section{Instrumentation}

This research was a narrative inquiry, which allowed the researcher to focus on the subjective experiences of the teachers of low-income students and their lived experiences in order to apply their experiences to the research connected to hope, pedagogy, and paradigm and the influence on student engagement. The questions the researcher asked of the Participants were open-ended questions, which enabled the researcher to explore the lived experiences of the Participants as they use their voices to tell their story (Seidman, 2006). The researcher used Seidman's three-tiered approach to interviews, in intervals of 30-45 minutes, spaced three days apart (Seidman, 2006). The first interview focused on the Participant as a teacher. The second interview focused on the details and experiences as a teacher related to hope in a low-income school. The third interview focused on the reflection of the first two interviews and the meaning provided through the answers that the Participants gave. Since this was a narrative inquiry, the questions asked in the second and third interview changed based on the stories from the first interview and the nature of the study method. While there were questions available for the second and third interview for all 10 Participants, some or none of the questions were asked as each Participant's story was different which required different questions for the second and third interview. See the interview questions in Figure 11. In addition to the interview questions, the observation tool the researcher used in determining how the elements of hope were manifested in 
teachers' paradigm and pedagogy thorough their language and behavior of hope, their learning tasks of hope and their lesson plans of hope.

During all interviews, audiotaping of the Participants was completed with the individualized consent and approval from Participants to ensure accurate transcriptions of their voices and the data that was being collected and analyzed to ensure a direct reflection of the inherent meaning. If interviewees declined the audiotaping, the primary investigator transcribed their answers during the interviews and read the answers back to the Participants to ensure the accuracy of the transcriptions. All interviewees were assigned alphanumeric codes to ensure protection of the Participants' identities during interviews and audiotaping. When discussing the findings and reporting data, only the alphanumeric codes will be used.

\section{Hope Survey}

\section{What is Your HOPE Score?}

Dr. Shane Lopez (2013), writer of Making Hope Happen has constructed a brief Hope Scale to measure your current level of hope.

Directions: Read each item carefully. Using the scale next to each item, ranging from Strongly Disagree (SD) to Strongly Agree (SA), please circle the number that best describes you.

\begin{tabular}{|c|c|}
\hline The Hope Scale & (SD) \\
\hline 1. My future will be better than the present. & 12345 \\
\hline 2. I have the power to make my future better. & 12345 \\
\hline 3. I am excited about at least one thing in my future. & 12345 \\
\hline 4. I see many paths to my goals. & 12345 \\
\hline 5. The paths to my important goals are free of obstacles. istept] & 12345 \\
\hline 6. My present life circumstances are the only determinants & 12345 \\
\hline
\end{tabular}


EXAMINING TEACHERS' PARADIGM AND PEDAGOGY IN MANIFESTING CRITICAL HOPE IN LOW-INCOME MIDDLE SCHOOL

\begin{tabular}{|c|c|}
\hline 7. My past accomplishments are the only determinants of $\mathrm{m}$ & 12345 \\
\hline 8. I make others feel excited about the future. isceps & 12345 \\
\hline 9. I spread hope through modeling or support of others. & 12345 \\
\hline 10. I spread hope through the way I live my life. SSEP & 2345 \\
\hline
\end{tabular}

Now add up your "Yes" answers: This is your Hope Score.

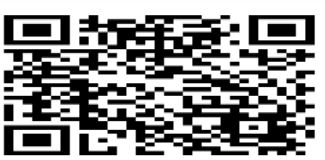

https:/qtrial2019q1az1.qualtrics.com/jfe/form/SV_9Rg8hkgwvCnz8q1

\section{Scoring:}

Questions 1-5: On a range from 5 to 25 , where did you score? This is your hope score. If it is below 15 , it will take hard work and much practice to raise that score. If you scored 16 to 20 , your hope is an asset to you every day, but here are many strate gies that can help you increase it. If you scored 21 or above, you are a high-hope person whose thinking about the future is an asset.

Questions 6-7: This is your readiness to Hope Score, ranging from 2 to 10. The higher you score, the more you believe that your future is dominated by your past and present circumstances, and the le ss room you have for hope. Learn to expand your sense of personal freedom without denying realistic constraints we all face. Take more control of the future.

Questions 8-10: This is your Hope Contagion score, ranging from 3 to 15. If you scored above 12 , you are a model for others and consciously boost the hope of those around you. A low score suggests that you would benefit greatly from seeking out the support and companionship of high-hope people in your daily life.

Figure 10: Hope Scale Survey 


\section{Interview Questions}

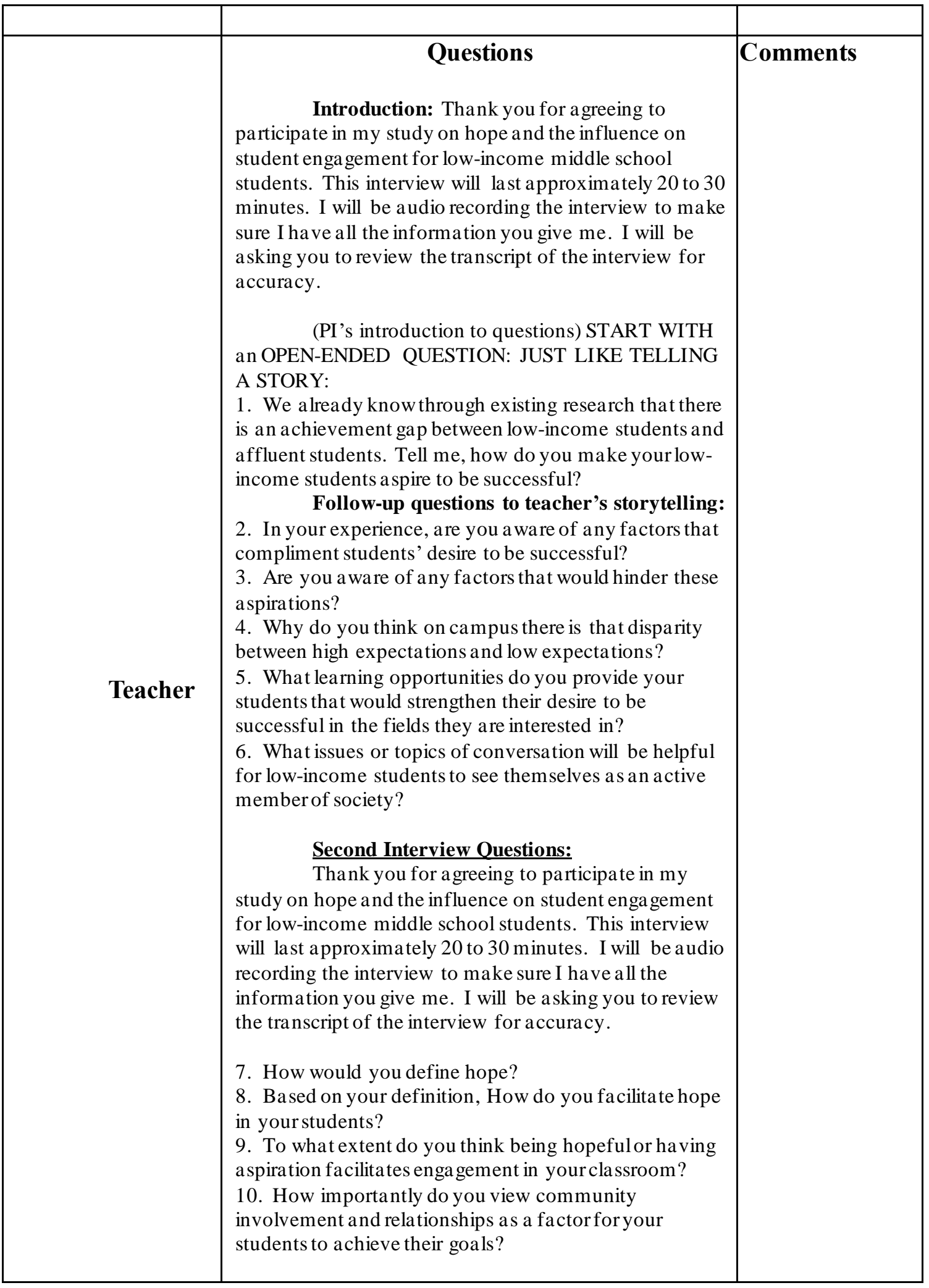




\begin{tabular}{|l|l|l|}
\hline \multicolumn{1}{|c|}{$\begin{array}{c}\text { Interview Three: } \\
\text { In this session you will be reviewing your } \\
\text { responses from the questions in interview one and two } \\
\text { by looking at the transcripts for any concerns. If there } \\
\text { are concerns, we will make some revisions. If there are } \\
\text { no concerns, we will proceed with data analysis. }\end{array}$} & \\
\hline
\end{tabular}

Figure 11: Interview Questions

\section{Participant Consent Letter}

Study Title: Examining Teachers' Paradigm and Pedagogy in Manifesting Critical Hope in LowIncome Middle School Classrooms: A Narrative Inquiry

Thank you for participating in this study. Your participation in this study is extremely valuable. I would like to take an opportunity to explain the purpose of this study in detail. This study was designed to gain insight into the lived experiences of teachers of low-income students to examine the manifestations of Critical Hope in teachers' paradigm and pedagogy reflected in low-income middle school classrooms. The study may result in stories for teachers, administrators, and policy makers to be able to listen and understand the perspectives of teachers of children of poverty whose voices and experiences related to hope have been silenced or disregarded in the current system of education. Existing research on hope demonstrates that students who develop high levels of hope engage in school and persevere through tasks better leading toward academic achievement, and students who have low er levels of hope are more likely to disengage from school and have negative school outcomes.

During this qualitative research, I will collect data by completing a series of interviews and observations with individuals who work inside a low-income middle school. These semistructured interviews and observations will be analyzed for common themes and experiences to 
better understand the multiple perspectives of teachers' paradigm and pedagogy related to hope. I am interested in using the information to help inform teachers, administrators, and policy makers on the manifestations of hope in teachers' paradigm and pedagogy reflected in classroom practices.

If any portion of this interview process was overly distressing or caused unresolved issues related to working in a trauma-sensitive school with low-income students, please seek help from a trained professional. You may contact the Mental Health Services Hotline, which has counselors available 24 hours a day, seven days a week at 800-854-7771.

In the future, should any questions arise regarding this study, please feel free to contact me. My dissertation advisor's contact information is Jose Lalas@redlands.edu If you would like a copy of the results of this study, I can send you a copy once they are approved by my advisor.

Thank you for your participation in this study,

Heidi Strikwerda

Figure 12: Participant Letter

\section{Manifestations of Critical Hope Reflected in Teachers' Paradigm and Pedagogy Observation Matrix}

(For observing elements of Critical Hope manifested in teachers' language and behavior of hope, teachers' instructional delivery of hope, and teachers' lesson panning of hope)

I. The Teacher

Time Observed:

Observer:

Date:

\begin{tabular}{|c|c|c|c|c|}
\hline $\begin{array}{l}\text { Manifestations of } \\
\text { Critical Hope } \\
\text { Elements in } \\
\text { Teachers' Paradigm }\end{array}$ & $\begin{array}{l}\text { Manifestations } \\
\text { of Critical } \\
\text { Hope in: }\end{array}$ & $\begin{array}{l}\text { General } \\
\text { Indicators }\end{array}$ & Examples & $\begin{array}{l}\text { Observed } \\
\text { Evidence }\end{array}$ \\
\hline
\end{tabular}




\begin{tabular}{|c|c|c|c|c|}
\hline \& Pedagogy & & & & \\
\hline 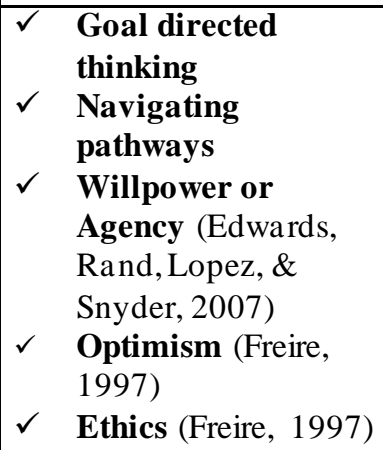 & $\begin{array}{l}\text { Teachers use } \\
\text { of vocabulary } \\
\text { expression } \\
\text { and written } \\
\text { directions }\end{array}$ & $\begin{array}{l}\text { - Language and } \\
\text { behavior that } \\
\text { connect, connote, } \\
\text { imply valuing and } \\
\text { trusting optimist } \\
\text { aspirations }\end{array}$ & 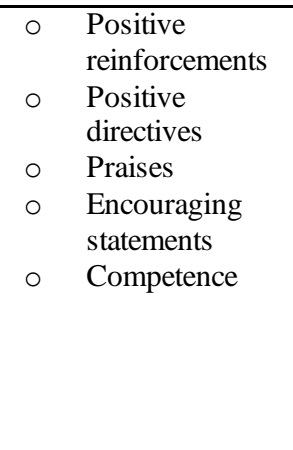 & \\
\hline $\begin{array}{ll}\checkmark & \text { Criticality (Freire, } \\
& \text { 1994, 1997) } \\
\checkmark & \text { Social and } \\
& \text { Historical } \\
\text { Consciousness } \\
\\
\text { (Freire, 1974, 1997) } \\
\text { Humanizing } \\
\text { Practices (Freire, } \\
\text { 1970, 1974, 1994, } \\
\text { 1997) }\end{array}$ & $\begin{array}{l}\text { Teachers } \\
\text { prepared } \\
\text { written tasks } \\
\text { and } \\
\text { assignments }\end{array}$ & $\begin{array}{l}\text { - Teacher delivery } \\
\text { in prompting } \\
\text { critical thinking } \\
\text { and aspirations to } \\
\text { be caring socially } \\
\text { conscious and } \\
\text { ethical }\end{array}$ & $\begin{array}{ll}\circ & \text { Higher levels of } \\
\text { questioning } \\
\text { Making value } \\
\text { judgments that } \\
\text { show caring and } \\
\text { ethical } \\
\text { dispositions } \\
\text { based upon the } \\
\text { criteria }\end{array}$ & \\
\hline $\begin{array}{ll}\checkmark & \begin{array}{l}\text { Sense of } \\
\text { Community }\end{array} \\
& \text { (hooks, 2003; Freire, } \\
1997) & \text { Caring } \\
\checkmark & \text { relationships } \\
& \text { (Noddings, 2017; } \\
& \text { Freire, 1997) } \\
\checkmark & \begin{array}{l}\text { Knowledge of Social } \\
\text { \& Cultural Capital }\end{array} \\
& \text { (Bourdieu, 1986) }\end{array}$ & $\begin{array}{l}\text { Teachers } \\
\text { composed } \\
\text { lesson plans. }\end{array}$ & $\begin{array}{l}\text { - Teacher planning } \\
\text { that may elicit a } \\
\text { sense of } \\
\text { community and } \\
\text { humanizing } \\
\text { practices }\end{array}$ & $\begin{array}{l}\text { Lesson plans that } \\
\text { require } \\
\text { collaboration and } \\
\text { creativity in } \\
\text { manifesting voice } \\
\text { and advocacy } \\
\text { that reflect sense } \\
\text { of community } \\
\text { and humanizing } \\
\text { practices. }\end{array}$ & \\
\hline
\end{tabular}

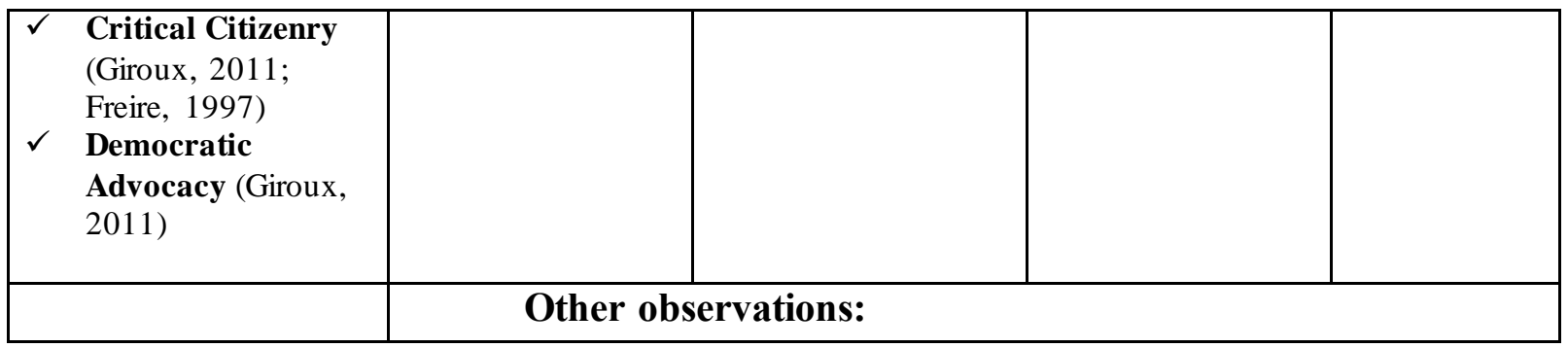

Figure 13: Manifestations of Critical Hope in Teachers' Paradigm and Pedagogy Observation Matrix Tool 
EXAMINING TEACHERS' PARADIGM AND PEDAGOGY IN MANIFESTING CRITICAL HOPE IN LOW-INCOME MIDDLE SCHOOL

\section{Data Collection Analysis}

The qualitative data from the narrative inquiry methodology was collected through three semi-structured interviews of 10 teachers for 30 to 45 minutes each in length, observations, and other relevant available information such as lesson plans, student work, and test data. The data gathering was completed with information from the interview transcriptions, field notes, and other observations. The primary investigator transcribed the interviews. The interview documents, field notes, and other observed data was read and analyzed to determine the preliminary codes based from the responses of the Participants. Primarily, hand coding was used to provide an immersed and engaged understanding of the lived experiences of all the Participants that were generated from interviews and observation. This will ensure that the researcher is grounded in the perspective and the voices of the Participants capturing the themes that emerge.

Once the preliminary codes and themes were developed, the researcher asked her dissertation chair, Dr. Jose Lalas, who is knowledgeable and experienced in narrative inquiry, to do a peer check and review about findings and themes that emerge from the conducted interviews and observations. This helped to eliminate researcher bias and to validate that the findings were accurate (Creswell \& Poth, 2018). In additon, the information from the preliminary data analysis was taken back to the Participants to complete the process with a check and review by them to ensure that the information that is being provided reflected the Participants' voices and the inherent meanings being shared. The sample size for narrative inquiry was 10 teachers, which is a small sample size. In order to collect extensive data, there were three interviews conducted of 30 to $45 \mathrm{~min}$ each (Seidman, 2006). The stories of the 
EXAMINING TEACHERS' PARADIGM AND PEDAGOGY IN MANIFESTING CRITICAL HOPE IN LOW-INCOME MIDDLE SCHOOL

Participants will add to the literature on hope in relation to pedagogy and paradigm as it is viewed through the perspectives of educators of low-income students.

\section{Summary}

Low-income communities face many hardships and recognizing these hardships and their influences on students can provide the community with hope. Numerous quantities of research exist in the medical field on the need for building hope when people face trauma (Lopez, 2013). The research demonstrated that those who are hopeful achieve their goals more often than those who are hopeless. When students enter a classroom feeling hopeless about their future, they often exhibit negative feelings and unwanted behaviors. It is important to have communication between the teachers, the students, and the parents regarding hope and setting goals, creating pathways to achieve those goals, and the support that is needed along the way. These actions can create hope in those who feel hopeless and can change the outcomes of their present lives leading towards future successes. Therefore, learning the importance of hopeful educational practices and pedagogy can be beneficial to all the stakeholders inside the school community. Specifically, professional development for teachers, administrators, and policy makers on the influence of hope on student engagement was formulated and will be offered.

While information regarding hopeful practices for low-income students continues to be a need in education, currently there is little information regarding the manifestations of Critical Hope in teachers' paradigm and pedagogy. This study will help to generate data regarding how teacher pedagogy and paradigm manifest hope for students quest for success in a low-income middle school.

The practical and educational benefits of this research include advancing my expertise on the manifestations of Critical Hope in teachers' paradigm that influence their pedagogy and 
practices inside the classroom. Hopelessness can occur due to a variety of reasons including broken homes, poverty, traumas, death, lack of support, and more. As a result, it will be beneficial to gain information on how to work with students in low-income schools who experience many different adverse childhood experiences, which decreases their hope and achievement.

In using narrative inquiry, this dissertation sought to bring authentic teachers' voices to the forefront through storytelling so that they become a part of the educational literature on hope, pedagogy, and paradigm and their connection to student engagement.

\section{Research Questions}

1. To what extent do the voices of teachers of low-income students reflect Critical Hope in their paradigm and pedagogy?

2. What themes related to teachers' language and behavior of hope, teachers' instructional delivery of hope, and teachers' lesson planning of hope emerge when observing and listening to teachers?

3. What recommendations for educators can be made to reflect the manifestations of “Critical Hope" in teachers' paradigm and pedagogy?

These research questions will give voice to teachers of low-income students who are often overshadowed, or left out of the discussion, but who have the lowest student outcomes of all sub groups (Flint, 2018). The rationale is to give teachers in low-income middle school the forum to manifest their understanding of Critical Hope in their paradigm and pedagogy. A qualitative methodological approach using narrative inquiry was selected to seek answers to the

research questions used to study, understand, and reconstruct the lived experiences of teachers in the realm of practice, while delving into conceptual exploration (Conle, 2001) and to provide 
EXAMINING TEACHERS' PARADIGM AND PEDAGOGY IN MANIFESTING CRITICAL HOPE IN LOW-INCOME MIDDLE SCHOOL

recommendations for teachers and policy makers who can become agents of hope in creating equitable practices and policies that may increase hope and engagement in low-income schools. 
EXAMINING TEACHERS' PARADIGM AND PEDAGOGY IN MANIFESTING CRITICAL HOPE IN LOW-INCOME MIDDLE SCHOOL

\section{Chapter Four. Data Analysis and Findings}

\section{Purpose}

The purpose of this research study was to answer the following research questions through a narrative inquiry methodology of 10 teachers' authentic voices: To what extents do the voices of teachers of low-income students reflect Critical Hope in their paradigm and pedagogy; What themes related to teachers' language and behavior of hope, teachers instructional delivery of hope, teachers' instructional planning of hope emerge when observing and listening to teachers; What recommendations for educators can be made to reflect the manifestations of “Critical Hope" in teachers' paradigm and pedagogy. The researcher used narrative inquiry method to interview 10 teachers of low-income middle school students over the course of twoweeks. This chapter includes a discussion of the findings from those interviews. The researcher first discusses the process used to organize the data for analysis to determine themes, and then discusses each theme and subsequent categories as they related to manifestations of Critical Hope in teachers' paradigm and pedagogy. The researcher provides a narrative story of each of the 10 teacher Participants demonstrating how the findings from the interviews addressed each of the three research questions. A completed conceptual map follows based on the data analysis providing a visual representation of how the findings relate to and added to the Critical Hope conceptual framework discussed in chapters one and two.

\section{Data Analysis}

\section{Process}

The researcher used the following process to organize the data collected from the 10 interviews. 
1. Data organization- organizing the 10 transcripts into data files.

2. Reading and memoing- read through each of the 10 transcripts to do initial codes and make notes in the margins and memos.

3. Describing the data into codes and themes- Describe the story of each Participant in chronology or a set of experiences

4. Classifying the data and codes into themes- Identify themes and categories in the stories, locate epiphanies to add to the conceptual framework.

5. Interpreting the data- Determine the meaning of data

6. Using Nvivo analysis as tool- upload data into computer software program.

7. Discovered and analyzed incidental findings

8. Dissertation chair did a peer review by going through pieces of the data and looked for themes and categories

9. Interpreting the data after triangulation- Determine meaning and locating epiphanies to add to conceptual framework and to the incidental findings.

10. Representing, visualizing the data into tables per theme describing the themes, categories, and elements of Critical Hope and providing evidence from the data to support the language and behavior of hope, the instructional delivery of hope, the planning of hope, and the paradigm of hope. A second table was created to show the themes related to Critical Hope with evidence from teachers' voices to support each and the connection to the elements of Critical Hope.

11. Incidental findings were found on hopelessness and also put into tables describing the themes, categories, and elements of hopelessness and provide evidence from the data to support the language and behavior of hopelessness, the instructional delivery of 
hopelessness, the planning of hopelessness, and the paradigm of hopelessness that emerged. A second table was created to show the themes related to hopelessness with evidence from teachers' voices to support each and the connection to the elements of hopelessness.

12. A second set of incidental findings were found on the zone of difference between hope and hopeless that I call the hope gap. A table representing and visualizing the data into tables per themes of hope and hopelessness, describing elements of Critical Hope, and providing textual evidence from the teachers' voices to support the findings of the hope gap are also included.

13. Organize the story of each of the 10 Participants in chronology of when the interviews took place.

14. The researcher wrote the 10 narratives to describe each of the Participants and their unique experiences as a teacher in a low-income middle school. Each narrative was framed in the context related to Critical Hope and discussed the themes and Critical Hope elements as they emerged.

\section{Findings}

The researcher first transcribed each of the 10,30-45 minute interviews as the stories were being told and saved them on a password-protected file with each of the teacher's alphanumeric code and marked as Participant 1 Interview, Participant 2 Interview, Participant 3 Interview, and so on for each interview. The transcribed interview was then given to the Participant to check for credibility and to ensure that the transcriptions were accurate and reliable. Then the researcher conducted an observation on each of the Participants during one class period to observe the Participant pedagogy in order to examine manifestations of Critical 
EXAMINING TEACHERS' PARADIGM AND PEDAGOGY IN MANIFESTING CRITICAL HOPE IN LOW-INCOME MIDDLE SCHOOL

Hope in the teacher's language and behavior of hope and instructional delivery of hope by using an observation matrix created and adapted from research done by Lalas et al,. (2016). Then the observation matrix was transcribed and saved on a password-protected file with the teacher's alphanumeric code and marked as Participant 1 Observation, Participant 2 Observation, Participant 3 Observation, etc. Lesson plans that were delivered during the observation of each of the 10 Participants were collected, organized, and saved on a password-protected file with the teacher's alphanumeric code and marked as Participant 1 Lesson Plans, Participant 2 Lesson Plans, Participant 3 Lesson Plans, etc. For more information please see the index labeled "Participant Data", which includes all transcribed interviews and transcribed classroom observations that has been collected and organized by Participant.

After the researcher transcribed the interviews, conducted the observations, and collected the lesson plans and organized the data by Participant into password-protected files using alphanumeric codes, the researcher then read through each of the transcripts, observations, and lesson plans and used the NVivo hand coding process as the initial coding, being sure to write notes in the margin for unanswered questions or possible themes as they emerged from the coding process. The researcher then uploaded the 10 interviews into the NVivo software program and conducted a text and word query to determine the most common words and descriptions from all 10 interviews pertaining to the 10 interview questions that were asked. Then the researcher grouped the common words and descriptions into categories of commonalities. As the commonalities were emerging, the researcher noticed two overarching findings and hand coded the commonalities in relation to two overarching findings. The first one is pertaining to hope as in the language and behavior of hope, the instructional delivery of hope, the lesson planning of hope, and the paradigm of hope. Invasively, a second finding emerged 
pertaining to hopelessness as depicted in the language and behavior of hopelessness, the instructional delivery of hopelessness, the lesson planning of hopelessness, and the paradigm of hopelessness. Figure 14 includes the first coding of Manifestations of Hope in Teachers' Paradigm and Pedagogy. 


\section{Manifestations of Hope in Teachers' Paradigm and Pedagogy}

\begin{tabular}{|c|c|c|c|}
\hline Indicators & Evidence & Themes of Critical Hope & Elements of Critical Hope - \\
\hline Paradigm of Hope & $\begin{array}{l}\text { Asset based } \\
\text { Faith } \\
\text { Growth mindset } \\
\text { Possitive Mindset/Optimism/Possibilitites }\end{array}$ & \begin{tabular}{|l} 
Valuing Optimism \\
Valuing Optimism \\
Valuing Optimism \\
Valuing Optimism \\
\end{tabular} & $\begin{array}{l}\text { Optimism } \\
\text { Optimism } \\
\text { Agency } \\
\text { Optimism } \\
\end{array}$ \\
\hline Language and Behavior of Hope & $\begin{array}{l}\text { Building Relationships/Trust/Authentic/Buy In/Acceptence/Stability } \\
\text { Teacher Roles: Councelor, Disciplinarian, Mentor, Role Model } \\
\text { Visable -Teacher involvement/Investment } \\
\text { Modeling Behavior/Demonstartion/Models of Excellence from theCommunity } \\
\text { Citizenry/Respect } \\
\text { Community involvement/Community Service } \\
\text { Grit/Perseverance } \\
\text { Goals/Purpose/Visualize } \\
\text { Seff-Realization/ldentity Development/Recognition } \\
\text { Safe-Comfortable Environment/Lower Effective Filter/Conditions for Learning } \\
\text { Parent Involvement/High Expectations at Home } \\
\text { Fun/ humor } \\
\text { Inspiration/Motivation/Engagement } \\
\text { Accountability/Work Ethic/Professional Accountability } \\
\text { Relentless/Pushing/Consistently Reinforcing/Challenge/Whatever it Takes } \\
\text { Possitive Adult Attitude/New Start Each Day } \\
\text { Driven: What drives you? What is your why for teaching? }\end{array}$ & $\begin{array}{l}\text { Confronting Hopelessness } \\
\text { Confronting Hopelessness } \\
\text { Confronting Hopelessness } \\
\text { Confronting Hopelessness } \\
\text { Critical Reflection, Advocacy, Citizenry, and Empowerment } \\
\text { Critical Reflection, Advocacy, Citizenry, and Empowerment } \\
\text { Goal Oriented, Willingness to Act, and Persevere } \\
\text { Goal Oriented, Willingness to Act, and Persevere } \\
\text { Humanizing Self-Identity and Sense of Community } \\
\text { Humanizing Self-Identity and Sense of Community } \\
\text { Humanizing Self-Identity and Sense of Community } \\
\text { Humanizing Self-Identity and Sense of Community } \\
\text { Inspiring Motivation and Engagement } \\
\text { Personal Commitment to Ethical Responsibility } \\
\text { Personal Committment to Ethical Responsibility } \\
\text { Valuing Optimism } \\
\text { Personal Commitment to Ethical Responsibility } \\
\end{array}$ & $\begin{array}{l}\text { Humanizing Practices } \\
\text { Humanizing Practices } \\
\text { Social And Historical Consciousness } \\
\text { Social And Historical Consciousness } \\
\text { Critical Citizenry } \\
\text { Critical Citizenry } \\
\text { Agency } \\
\text { Goal Directed Thinking } \\
\text { Humanizing Practices } \\
\text { Sense of Community } \\
\text { Social \& Cultural Capital } \\
\text { Social \& Cultural Capital } \\
\text { Engagement } \\
\text { Ethics } \\
\text { Ethics } \\
\text { Optimism } \\
\text { Ethics }\end{array}$ \\
\hline Instructional Delivery of Hope & $\begin{array}{l}\text { Hopeful Climate/SEL/PBIS } \\
\text { Focussed on Learning/Student Centered/Teacher as a Facilitator } \\
\text { Culture of Goal Setting/Cycle of Goal/Futuristic Thinking/College Going Culture } \\
\text { Believe/True believer/Competency } \\
\text { Culturally Relevant } \\
\text { Criticality/Evidence Based/Analytical Thinking/Problem Posing } \\
\text { Independent Learners/Students have body of knowledge } \\
\text { Possitive feedback } \\
\text { Autonomy/Student Choice } \\
\text { Meaningfulness/Connection to Future Aspirations/Student Interest/Relevancy }\end{array}$ & $\begin{array}{l}\text { Valuing Optimism } \\
\text { Building Trust, High Expectations, and Caring Relationships } \\
\text { Goal Oriented, Willingness to Act, and Persevere } \\
\text { Inspiring Motivation and Engagement } \\
\text { Confronting Hopelessness } \\
\text { Critical Reflection, Advocacy, Citizenry, and Empowerment } \\
\text { Critical Reflection, Advocacy, Citizenry, and Empowerment } \\
\text { Critical Reflection, Advocacy, Citizenry, and Empowerment } \\
\text { Inspiring Motivation and Engagement } \\
\text { inspiring Motivation and Engaeement }\end{array}$ & $\begin{array}{l}\text { Sense of Community } \\
\text { Caring Relationships } \\
\text { Goal Directed Thinking } \\
\text { Engagement } \\
\text { Social And Historical Consciousness } \\
\text { Criticality } \\
\text { Criticality } \\
\text { Criticality } \\
\text { Engagement } \\
\text { Eneagement }\end{array}$ \\
\hline
\end{tabular}




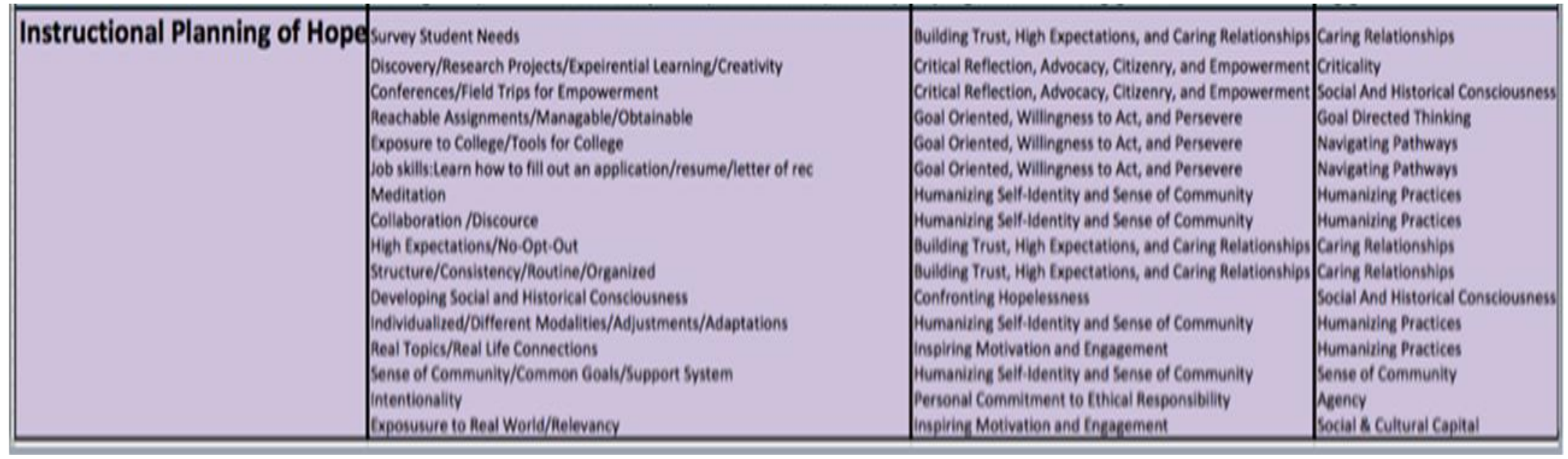

Figure 14: Manifestations of Hope in Teachers' Paradigm and Pedagogy 
EXAMINING TEACHERS' PARADIGM AND PEDAGOGY IN MANIFESTING CRITICAL HOPE IN LOW-INCOME MIDDLE CLASSROOM

First, the researcher hand coded all 10 transcripts pertaining to hope as in the language and behavior of hope, the instructional delivery of hope, the lesson planning of hope, and the paradigm of hope.

\section{Themes and Categories}

The themes identified using hand coding by the researcher led to the discovery of the manifestations of both positions of hope and hopelessness in teachers' paradigm and pedagogy. The following section describes each theme and category, as a unique and shared experience of each Participant, as it relates to the manifestations of Critical Hope in teachers' paradigm and pedagogy. Following each explanation is a table with a visual representation of each theme and category.

The interviews of all 10 Participants demonstrated the themes as manifestations of Critical Hope in teachers' paradigm and pedagogy. While some themes have more textual evidence than others, the unique experiences of the 10 teachers are described in detail in the narrative section of this chapter. Previously in this research, Critical Hope was defined as the optimistic way of viewing and acting in the world from a critically, historically conscious, socially and culturally situated perspective with the belief that inevitable change will inspire a sense of community, advocacy, liberation, and justice. During the interviews, the Participants were asked to define hope, "What does hope mean to you?" The researcher then took all 10 Participants' answers and put them into NVivo software to create a word wall of the Participants' definitions of hope (see Figure 15). 


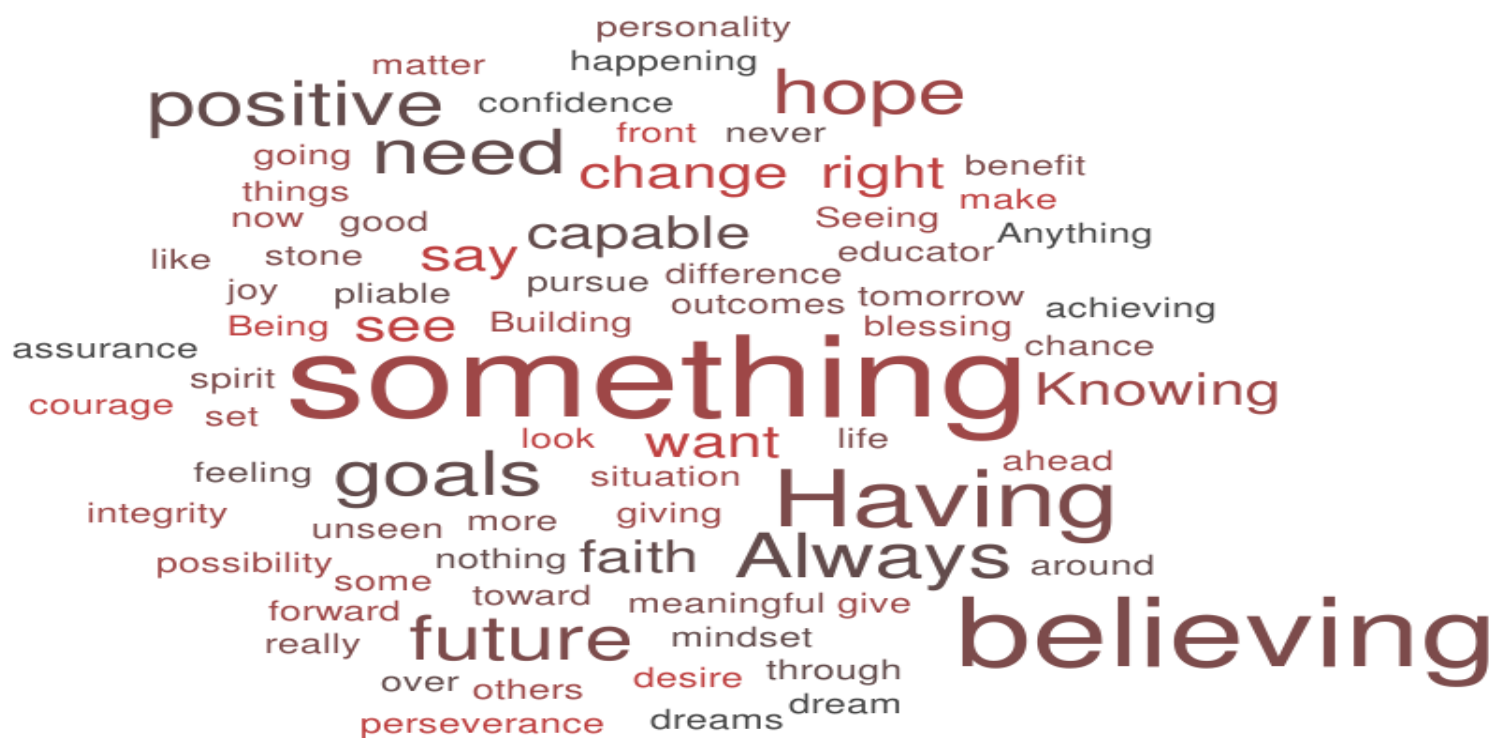

Figure 15: Words Related to Hope as Reflected by Teachers' Voices

After reviewing the teachers' voices as reflected in this word wall, I discovered that the most frequently used word to define hope is the word "something." This means that hope is very personal and is connected to a belief in something. Thus, I redefined Critical Hope to reflect the voices of the teachers and included a personal belief in the definition. Therefore, Critical Hope is defined as the optimistic way of viewing and acting on the world from a critically, historically conscious, socially and culturally situated perspective with a personal belief that inevitable change will inspire a sense of community, advocacy, liberation, and justice.

Then the researcher identified nine themes pertaining to Critical Hope as they emerged from the authentic voices of teachers and are consistent with the shared experiences of the teachers' in the low-income middle school.

1. Goal Oriented, Willingness to Act, and Perseverance

2. Building Trust, High Expectations, and Caring Relationships

3. Critical Reflection, Advocacy, Citizenry, and Empowerment 
4. Valuing optimism

5. Confronting Hopelessness

6. Personal Commitment to Ethical Responsibility and Accountability

7. Humanizing Self-Identity and Sense of Community

8. Consciousness of Economic, Social, Cultural, and Historical Life Barriers

9. Inspiring Motivation and Engagement

Using the nine themes of hope, the researcher created NVivo themes and coded all 10 interview transcripts. The researcher then read through all the transcriptions again and hand coded the transcripts looking for the textual evidence from the teachers' voices as it emerged in each of the nine themes of hope. 


\begin{tabular}{|c|c|c|}
\hline $\begin{array}{l}\text { Themes of Critical } \\
\text { Hope }\end{array}$ & $\begin{array}{l}\text { Elements of } \\
\text { Critical Hope }\end{array}$ & $\begin{array}{l}\text { Manifestations of Hope } \\
\text { (Teachers Voices) }\end{array}$ \\
\hline \multirow{10}{*}{$\begin{array}{l}\text { Goal Oriented, } \\
\text { Willingness to Act, } \\
\text { and Perseverance } \\
\text { Mentally invested, } \\
\text { engaged, focused on } \\
\text { achieving clear and } \\
\text { difficult goals. } \\
\text { Embraces barriers } \\
\text { and sees them as a } \\
\text { challenge. } \\
\text { Anticipates } \\
\text { problems, plans } \\
\text { ahead for roadblocks, } \\
\text { has patience with } \\
\text { unfolding events and } \\
\text { is mentally flexible. }\end{array}$} & \multirow{10}{*}{$\begin{array}{l}\text { - Agency } \\
\text { - Caring } \\
\text { Relationships } \\
\text { - Goal Directed } \\
\text { Thinking } \\
\text { - Navigating - } \\
\text { Pathways } \\
\text { - Optimism } \\
\text { - Social and } \\
\text { Cultural } \\
\text { Capital }\end{array}$} & "The hopeful students are working and completing tasks." \\
\hline & & "I build a culture of having a goal." \\
\hline & & $\begin{array}{l}\text { "We talk a lot with our kids about goal setting, their future careers, their } \\
\text { strengths, and what they are good at." }\end{array}$ \\
\hline & & $\begin{array}{l}\text { "With goal setting, with career day projects, I try to guide them towards } \\
\text { always having something specific they are working towards. So they } \\
\text { understand there is a cycle: set a goal, try, fail, keep trying, succeed, then } \\
\text { set a new goal. And we talk a lot about failure in class as a step toward } \\
\text { success." }\end{array}$ \\
\hline & & "If you have hope you will try." \\
\hline & & $\begin{array}{l}\text { "There are many factors that make people successful like grit, and when } \\
\text { we are working together for a common goal it changes the mindset like this } \\
\text { person believes in me." }\end{array}$ \\
\hline & & $\begin{array}{l}\text { "So I believe that if kids see that they can be successful then they are more } \\
\text { likely to try to be successful." }\end{array}$ \\
\hline & & $\begin{array}{l}\text { "You have to have plans and plans start now. There has to be a work ethic } \\
\text { to go with it. You might not want to go to college but it is work ethnic that } \\
\text { you need to be successful for your future." }\end{array}$ \\
\hline & & $\begin{array}{l}\text { "We talk a lot with our kids about goal setting, their future careers, their } \\
\text { strengths, and what they are good at." }\end{array}$ \\
\hline & & $\begin{array}{l}\text { "They are goal setting on their own now and telling us what they want to } \\
\text { do." }\end{array}$ \\
\hline
\end{tabular}

Figure 16: Goal Oriented, Willingness to Act, and Perseverance 


\begin{tabular}{|c|c|c|}
\hline \multirow{10}{*}{$\begin{array}{l}\text { Building Trust, } \\
\text { High Expectations, } \\
\text { and Caring } \\
\text { Relationships } \\
\text { Others centered, close } \\
\text { connection to } \\
\text { caregivers, enjoys } \\
\text { competition }\end{array}$} & \multirow{10}{*}{$\begin{array}{l}\text { - Caring } \\
\text { Relationships } \\
\text { - Sense of } \\
\text { Community } \\
\text { - Social and } \\
\text { Cultural } \\
\text { Capital } \\
\text { - Optimism } \\
\text { - Goal Directed } \\
\text { Thinking }\end{array}$} & $\begin{array}{l}\text { "I need them to visualize in the future, that's part of the hope. They have to } \\
\text { visualize in the future how much better they are going to be with me, if they } \\
\text { enogee with me as a teacher." }\end{array}$ \\
\hline & & $\begin{array}{l}\text { "Then I tell them to trust me and then I say, "Try! If you try and you trust me, } \\
\text { you will experience success." }\end{array}$ \\
\hline & & $\begin{array}{l}\text { "Not afraid to challenge scholars even when they say they can't do it even } \\
\text { though you know they can." }\end{array}$ \\
\hline & & "My constant reminder that I send them is they are capable, building them up." \\
\hline & & $\begin{array}{l}\text { "That girl, her mom has cancer she talks to me. I know you are going through } \\
\text { these things, I tell her you are working so hard and you will be able to take care } \\
\text { of her." }\end{array}$ \\
\hline & & $\begin{array}{l}\text { "They have to know that someone in in their corner and that someone is } \\
\text { expecting them to be successful. They see that you are relentless, that you are } \\
\text { not giving up, that they have no other option, than to meet that standard." }\end{array}$ \\
\hline & & $\begin{array}{l}\text { "I am a true believer in setting the expectations high and pushing my students } \\
\text { to be there best and helping along the way." }\end{array}$ \\
\hline & & $\begin{array}{l}\text { I think that finding a middle ground in the classroom where they are being } \\
\text { held to expectations, there are consequences, and at the same time there is a } \\
\text { new start we start over tomorrow. }\end{array}$ \\
\hline & & $\begin{array}{l}\text { "Some of them did not even sleep. Sometimes they tell me 'I have a headache, } \\
\text { so I let them put their head down for } 5 \text { min. You know what the student is } \\
\text { going to do for me? He will work for me because they know that I care more } \\
\text { about them, than just math." }\end{array}$ \\
\hline & & $\begin{array}{l}\text { "Relationships in my classroom are my bread and butter. My goal is to form a } \\
\text { relationship with them as well as with their guardians, their parents first } \\
\text { because that is where trust is built." }\end{array}$ \\
\hline
\end{tabular}

Figure 17: Building Trust, High Expectations, and Caring Relationships 


\begin{tabular}{|c|c|c|}
\hline $\begin{array}{l}\text { Themes of Critical } \\
\text { Hope }\end{array}$ & $\begin{array}{l}\text { Elements of } \\
\text { Critical Hope }\end{array}$ & $\begin{array}{l}\text { Manifestations of Hope } \\
\text { (Teachers Voices) }\end{array}$ \\
\hline \multirow{6}{*}{$\begin{array}{l}\text { Critical Reflection, } \\
\text { Advocacy, } \\
\text { Citizenry, and } \\
\text { Empowerment } \\
\text { Takes bold risks, } \\
\text { accepts challenges, } \\
\text { enjoys trying new } \\
\text { things and is attracted } \\
\text { to the process of testing } \\
\text { and stretching their } \\
\text { skills. }\end{array}$} & \multirow{6}{*}{$\begin{array}{l}\text { - Critical } \\
\text { Citizenry } \\
\text { - Criticality } \\
\text { - Democratic } \\
\text { Advocacy } \\
\text { - Social and } \\
\text { Historical } \\
\text { Consciousness }\end{array}$} & $\begin{array}{l}\text { "They annotate, research, I am pushing you hard, and if I give it to you, I } \\
\text { know you can do it." }\end{array}$ \\
\hline & & $\begin{array}{l}\text { "I think it goes with modeling behavior. If I want them to be respectful to } \\
\text { me, then I need to be respectful to them. If I want them to be involved in } \\
\text { the community, then I need to be involved. }\end{array}$ \\
\hline & & $\begin{array}{l}\text { "Just because you're } 13 \text { or } 14 \text { does not mean that you can't have an impact. } \\
\text { Think about voting, community service, help with siblings, homework, } \\
\text { anything that would help in your community, so you can see the positive } \\
\text { impact of giving. }\end{array}$ \\
\hline & & $\begin{array}{l}\text { "Not to be afraid to use your voice when we have discussions. Its ok to } \\
\text { have an opinion, but make sure with that opinion you have facts." }\end{array}$ \\
\hline & & $\begin{array}{l}\text { "I think topics of how in different ways people can make a difference and } \\
\text { that could range a lot. Even that could be a broad spectrum: like what does } \\
\text { that mean to make a difference. For example, how does pursuing a career } \\
\text { make an impact for someone else? How would you help others in this } \\
\text { career? What do your parents do and how do they make a difference in } \\
\text { their community? In some way or another we all affect each other." }\end{array}$ \\
\hline & & $\begin{array}{l}\text { "As much as I want them to keep their innocence and their culture, they } \\
\text { also have to know they have to have a voice because someone is going to } \\
\text { try to burst your bubble someday. You must know how to respond in these } \\
\text { situations. If you don't tell them, they will go out and set themselves up } \\
\text { for failure and get knocked off of their journey. It will take them a while } \\
\text { to come back. They need to know how others see their place is in society } \\
\text { and how you have to work against that place, and if no one ever tells you, } \\
\text { you do not know what you are working against." }\end{array}$ \\
\hline
\end{tabular}

Figure 18: Critical Reflection, Advocacy, Citizenry, and Empowerment 


\begin{tabular}{|c|c|c|}
\hline $\begin{array}{l}\text { Themes of Critical } \\
\text { Hope }\end{array}$ & $\begin{array}{l}\text { Elements of } \\
\text { Critical Hope }\end{array}$ & $\begin{array}{l}\text { Manifestations of Hope } \\
\text { (Teachers Voices) }\end{array}$ \\
\hline \multirow{13}{*}{$\begin{array}{c}\text { Valuing Optimism: } \\
\text { Sees setbacks as } \\
\text { momentary, Diminishes } \\
\text { setbacks, does not dwell } \\
\text { on past, failures motivat } \\
\text { them, and greater } \\
\text { happiness. }\end{array}$} & \multirow{13}{*}{$\begin{array}{l}\text { - Agency Goal } \\
\text { Directed } \\
\text { Thinking } \\
\text { - Optimism } \\
\text { - Sense of } \\
\text { Community } \\
\text { - Social \& } \\
\text { Cultural } \\
\text { Capital } \\
\text { - Caring } \\
\text { Relationship } \\
\text { - Humanizing }\end{array}$} & $\begin{array}{l}\text { "I try to approach them as already being successful because its hard to think of } \\
\text { ourselves that way." }\end{array}$ \\
\hline & & $\begin{array}{l}\text { "They are already successful, what do they need to be more successful than they } \\
\text { already are." }\end{array}$ \\
\hline & & $\begin{array}{l}\text { "I always try to highlight the positive. 'Look, you got } 2 \text { out of } 10 \text {, you already know } 2 \\
\text { things!" }\end{array}$ \\
\hline & & $\begin{array}{l}\text { "I try to help them have a positive mindset. If you think you can't do something, you } \\
\text { won't try. You can always picture what you want to do and your dreams will become } \\
\text { a reality someday }\end{array}$ \\
\hline & & $\begin{array}{l}\text { "One of the conversations I have with the } 8^{\text {th }} \text { graders before they leave me has to do } \\
\text { with their future. I tell them, "When you leave that University, I want a pairing flag } \\
\text { from your University because your success is my success." }\end{array}$ \\
\hline & & $\begin{array}{l}\text { "I try to help them realize that they are one step closer to figuring out what they want } \\
\text { to do by discovering that this career isn't for me." }\end{array}$ \\
\hline & & "I would be constantly reinforcing that I believe in them." \\
\hline & & $\begin{array}{l}\text { "Me believing in them, and them believing in themselves. My belief in them, } 50 \text { it } \\
\text { translates into them believing in themselves." }\end{array}$ \\
\hline & & "I try to finds their strengths and skills and give them positive feedback on that." \\
\hline & & $\begin{array}{l}\text { "Community involvement, when it is positive, it is very important. When you think } \\
\text { about the community they live around here, there is a lot of negativity. It is important } \\
\text { to plug into the positives." }\end{array}$ \\
\hline & & $\begin{array}{l}\text { "Always talking to them and telling them that you are capable and you are able just } \\
\text { as much as anyone else. I think those things are helpful." }\end{array}$ \\
\hline & & "My thing is they are kids and they start new everyday with me." \\
\hline & & $\begin{array}{l}\text { "Giving them what they need to help them be successful. It does not happen over } \\
\text { night." }\end{array}$ \\
\hline
\end{tabular}

Figure 19: Valuing Optimism 


\begin{tabular}{|c|c|c|}
\hline $\begin{array}{c}\text { Themes of Critical } \\
\text { Hope }\end{array}$ & $\begin{array}{l}\text { Elements of } \\
\text { Critical Hope }\end{array}$ & $\begin{array}{l}\text { Manifestations of Hope } \\
\text { (Teachers Voices) }\end{array}$ \\
\hline \multirow{6}{*}{$\begin{array}{c}\text { Confronting } \\
\text { Hopelessness } \\
\text { Through relevant and } \\
\text { authentic personal } \\
\text { narratives, relentless } \\
\text { belief in their } \\
\text { capabilities and } \\
\text { understanding }\end{array}$} & \multirow{6}{*}{$\begin{array}{l}\text { - Humanizing } \\
\text { Practices } \\
\text { - Social and } \\
\text { Historical } \\
\text { Consciousness } \\
\text { - Caring } \\
\text { Relationships }\end{array}$} & $\begin{array}{l}\text { "Not afraid to challenge scholars even when they say they can't do it even } \\
\text { though you know they can." }\end{array}$ \\
\hline & & $\begin{array}{l}\text { "I try to encourage them. I don't want them to look at their present } \\
\text { circumstances. I don't care where you came from. I don't want you to say } \\
\text { I did not learn this because I came from King; I want them to learn this } \\
\text { well. They don't have to go to a private school in a rich area; they can be } \\
\text { successful right where they are..." }\end{array}$ \\
\hline & & $\begin{array}{l}\text { "I try to use their stories, to turn it around on how they can be great, to } \\
\text { empower them because someone might use their story to demote them and } \\
\text { make them feel less than worthy to be on this earth. I take that very same } \\
\text { story and turn it around, to help them feel like they have worth." }\end{array}$ \\
\hline & & $\begin{array}{l}\text { "I try to be a model, an example to them. Throughout the year I tell them } \\
\text { my own personal stories that relate to them. How I got to this point How I } \\
\text { made my way. Things were not set up for me to be successful and through } \\
\text { my family and my faith I made myself successful and they can do that } \\
\text { too." }\end{array}$ \\
\hline & & $\begin{array}{l}\text { "I try to give them stories about the things I have done, about people I } \\
\text { know, real life examples to make things relevant and to let them know that } \\
\text { where you live doesn't define where you are going." }\end{array}$ \\
\hline & & $\begin{array}{l}\text { "By making them see how I relate to them, by making them see how I } \\
\text { came from a similar background and making them see, for them to feel } \\
\text { successful to see how I grew up and my parents grew up. If they were to } \\
\text { see where I came from, the neighborhood, the poverty, the discrimination } \\
\text { to where I am now, they can see that hope and inspiration for them to see } \\
\text { that you can be successful in an upbringing that is similar to mine." }\end{array}$ \\
\hline
\end{tabular}

Figure 20: Confronting Hopelessness 


\begin{tabular}{|c|c|c|}
\hline $\begin{array}{c}\text { Themes of Critical } \\
\text { Hope }\end{array}$ & $\begin{array}{l}\text { Elements of } \\
\text { Critical Hope }\end{array}$ & $\begin{array}{l}\text { Manifestations of Hope } \\
\text { (Teachers Voices) }\end{array}$ \\
\hline \multirow{6}{*}{$\begin{array}{l}\text { Personal } \\
\text { Commitment to } \\
\text { Ethical } \\
\text { Responsibility and } \\
\text { Accountability: } \\
\text { Positive adult attitude, } \\
\text { and holds themselves } \\
\text { professionally and } \\
\text { personally responsible } \\
\text { to whatever it takes } \\
\text { ensure student's } \\
\text { success. }\end{array}$} & \multirow{6}{*}{$\begin{array}{l}\text { - Agency } \\
\text { - Ethics } \\
\text { - Sense of } \\
\text { Community }\end{array}$} & $\begin{array}{l}\text { "I find myself being a counselor, a teacher, disciplinarian, many other roles } \\
\text { in middle school." }\end{array}$ \\
\hline & & $\begin{array}{l}\text { "I think it has to do with more of you as a person. What is your goal? What } \\
\text { is your motive for teaching? Your why? If my why is to see my students } \\
\text { succeed then I know that I will have high expectations. If they are not } \\
\text { learning the responsibility involves me." }\end{array}$ \\
\hline & & $\begin{array}{l}\text { "Demonstration first! I have to have faith, so I have to have hope and } \\
\text { confidence in them, in their abilities first because a lot of them do not } \\
\text { believe in themselves. I have to have almost an exaggerated amount of } \\
\text { hope, so they buy in." }\end{array}$ \\
\hline & & $\begin{array}{l}\text { "In general, show you want to be more than just a teacher, be active in the } \\
\text { community, a part of their culture in the community, in the school, and be } \\
\text { hopeful. That is when you have complete buy in. It's hard but once you } \\
\text { get the parents trust that opens the doors for every thing else." }\end{array}$ \\
\hline & & $\begin{array}{l}\text { Even when they do something small, I have to show them and make it a } \\
\text { big deal. Wow!!!This is amazing!! Wow!! Then after a few months they } \\
\text { start using my language. I have to make sure that my jar of hope is full } \\
\text { and running over because they might not see hope in their homes, or on } \\
\text { the street. I have to be that demonstration, that ray of light, when everyone } \\
\text { who is around them is telling them something different." }\end{array}$ \\
\hline & & $\begin{array}{l}\text { "If they are hungry, I can choose to provide something for them. When I } \\
\text { bring all these oranges I don't bring them just for myself. I know I have } \\
\text { students who haven't eaten The granola bars; the students have been eating } \\
\text { them because they don't have breakfast. Making sure they are eating well." }\end{array}$ \\
\hline
\end{tabular}

Figure 21: Personal Commitment to Ethical Responsibility and Accountability 


\begin{tabular}{|c|c|c|}
\hline $\begin{array}{l}\text { Themes of Critical } \\
\text { Hope }\end{array}$ & $\begin{array}{l}\text { Elements of } \\
\text { Critical Hope }\end{array}$ & $\begin{array}{l}\text { Manifestations of Hope } \\
\text { (Teachers Voices) }\end{array}$ \\
\hline \multirow{9}{*}{$\begin{array}{l}\text { Humanizing Self } \\
\text { Identity and } \\
\text { Sense of } \\
\text { community } \\
\text { Builds self-esteem and } \\
\text { self-efficacy as a } \\
\text { result of efficiency in } \\
\text { pathways and agency } \\
\text { needed to reach and } \\
\text { attain past goals. }\end{array}$} & \multirow{9}{*}{$\begin{array}{l}\text { - Humanizing } \\
\text { Practices } \\
\text { - Optimism } \\
\text { - Sense of } \\
\text { Community } \\
\text { - Social \& } \\
\text { Cultural } \\
\text { Capital } \\
\text { - Goal Directed } \\
\text { Thinking } \\
\text { - Visualized } \\
\text { Pathways } \\
\text { - Caring } \\
\text { Relationships }\end{array}$} & $\begin{array}{l}\text { "I try to start with what they are doing well and push them a little further. } \\
\text { Instead of starting with what they are doing wrong. I try to focus on what } \\
\text { they are doing right." }\end{array}$ \\
\hline & & $\begin{array}{l}\text { "By giving them support by building confidence in them that they can } \\
\text { achieve whatever they put their mind into. Positive reinforcement." }\end{array}$ \\
\hline & & $\begin{array}{l}\text { "Me believing in them, and them believing in themselves. My belief in } \\
\text { them, so it translates into them believing in themselves." }\end{array}$ \\
\hline & & $\begin{array}{l}\text { "No opt out. When they say I don't know, IDK, letting them not be afraid } \\
\text { to try. Even if the answer is not correct, showing them that they can learn } \\
\text { the information whether it is at this school, or another school. Your belief } \\
\text { is what builds that confidence in that scholar." }\end{array}$ \\
\hline & & "I constantly reinforcing that I believe in them." \\
\hline & & $\begin{array}{l}\text { "In projects they find their nitch because it has a writing component, an } \\
\text { art component and a creative component in different modalities, and they } \\
\text { learn they are good at things." }\end{array}$ \\
\hline & & $\begin{array}{l}\text { "Once the trust is built, I can teach and I can teach effectively because I } \\
\text { am a human being and I can have honest conversations and admit that I } \\
\text { have done something wrong." }\end{array}$ \\
\hline & & $\begin{array}{l}\text { "When they ask me questions, I tell them to find out and then come and } \\
\text { teach me. Everyone here, all the resources, that I am not the only one with } \\
\text { the body of knowledge. I tell them to ask the people in this room." }\end{array}$ \\
\hline & & $\begin{array}{l}\text { "Behaviors can be outrageous and personally offensive, but at the end of } \\
\text { the day they are an expression of a need that is not being met. If you can } \\
\text { connect it to that, you can move passed the behavior and into the person." } \\
\text { | }\end{array}$ \\
\hline
\end{tabular}

Figure 22: Humanizing Self-Identity and Sense of Community 


\begin{tabular}{|c|c|c|}
\hline $\begin{array}{c}\text { Themes of Critical } \\
\text { Hope }\end{array}$ & $\begin{array}{l}\text { Elements of } \\
\text { Critical Hope }\end{array}$ & $\begin{array}{l}\text { Manifestations of Hope } \\
\text { (Teachers Voices) }\end{array}$ \\
\hline \multirow{8}{*}{$\begin{array}{l}\text { Consciousness of } \\
\text { Economic, Social, } \\
\text { Cultural, and } \\
\text { Historical Life } \\
\text { Barriers } \\
\text { Awareness of systemic } \\
\text { oppression and } \\
\text { personal barriers in } \\
\text { students' daily lives. }\end{array}$} & \multirow{8}{*}{$\begin{array}{l}\text { - Navigating } \\
\text { Pathways } \\
\text { - Social And } \\
\text { Historical } \\
\text { Consciousness } \\
\text { - Sense of } \\
\text { Community }\end{array}$} & $\begin{array}{l}\text { "As educators, I think we need to be aware of those factors. There are a lot of } \\
\text { barriers they have to overcome in our community being low socio-economic } \\
\text { and a lot of them come with issues that you would not expect of middle- } \\
\text { schoolers." }\end{array}$ \\
\hline & & $\begin{array}{l}\text { "Then I tell them that the only way that San Bernardino gets better is if we } \\
\text { keep our best and you are our best. While I want you to go out and live your } \\
\text { dreams and experience life to the fullest, I want you to come back here because } \\
\text { this is the environment that made you who you are. Its really about having that } \\
\text { honest conversation." }\end{array}$ \\
\hline & & "A lot of them have tough lives at home and they share that with me." \\
\hline & & "I try to be honest and give them statistics about prison, about being a target." \\
\hline & & $\begin{array}{l}\text { "I was going over the statistics on how I and } 6 \text { Latino males go to prison and } \\
\text { how those who do not graduate often go to prison and I related that to him and } \\
\text { his grades and his effort. I told him that I do not want one of my students to go } \\
\text { to prison, but its on you." }\end{array}$ \\
\hline & & $\begin{array}{l}\text { "The school is really an extension of the community. If you are not involved it } \\
\text { will impact the school. Whatever problems you see in the community, you will } \\
\text { see in the school. You have to be involved in the community so you all can be } \\
\text { on the same page." }\end{array}$ \\
\hline & & $\begin{array}{l}\text { "They do not have any real example; they do not have anyone to help them at } \\
\text { home who can tutor or to help them. These kids do no have access to that. For a } \\
\text { lot of them it's not an option to stay after school because of safety." }\end{array}$ \\
\hline & & "Their well-being can hinder their aspirations too." \\
\hline
\end{tabular}

Figure 23: Consciousness of Economic, Social, Cultural, and Historical Life Barriers 


\begin{tabular}{|c|c|c|}
\hline $\begin{array}{c}\text { Themes of Critical } \\
\text { Hope }\end{array}$ & $\begin{array}{l}\text { Elements of } \\
\text { Critical Hope }\end{array}$ & $\begin{array}{l}\text { Manifestations of Hope } \\
\text { (Teachers Voices) }\end{array}$ \\
\hline \multirow{10}{*}{\begin{tabular}{|c|} 
Inspiring \\
Motivation and \\
Engagement \\
Less burnout, grit, \\
perseverance, high task \\
completion, active in \\
their thinking, meets \\
deadlines.
\end{tabular}} & \multirow{10}{*}{$\begin{array}{l}\text { - Engagement } \\
\text { - Humanizing } \\
\text { Practices } \\
\text { - Social \& } \\
\text { Cultural } \\
\text { Capital } \\
\text { - Social \& } \\
\text { Historical } \\
\text { Consciousness }\end{array}$} & $\begin{array}{l}\text { "When they experience success outside my four walls that is when their } \\
\text { desire for success grows tenfold. Once they experience some success it is } \\
\text { contagious and they want to try harder." }\end{array}$ \\
\hline & & $\begin{array}{l}\text { "I have to be that demonstration, that ray of light, when everyone who is } \\
\text { around them is telling them something different." }\end{array}$ \\
\hline & & $\begin{array}{l}\text { "By finding ways to get them interested in what you are doing and } \\
\text { celebrating little small success." }\end{array}$ \\
\hline & & $\begin{array}{l}\text { "Talking about things that motivate them. Some of the things they want } \\
\text { to see incorporated in the classroom. Allowing them to have a voice, } \\
\text { build a community where that can have input on issues." }\end{array}$ \\
\hline & & $\begin{array}{l}\text { "As far as in class, I try to give them the freedom of choice in some } \\
\text { projects we do, that way they can take control of their learning. We do a } \\
\text { lot of hands on. I love hands on." }\end{array}$ \\
\hline & & $\begin{array}{l}\text { "That is what triggers the students, They realize real quick. This again is } \\
\text { without me. They do their own research. They discover." }\end{array}$ \\
\hline & & $\begin{array}{l}\text { "Not one thing works for all kids. Experience little success and once } \\
\text { "they experience a little success it propels them to want to do more." }\end{array}$ \\
\hline & & "I think hope is the biggest factor when it comes to engagement." \\
\hline & & $\begin{array}{l}\text { "Acknowledging them and making them feel valued in the classroom and } \\
\text { even out of the classroom like going to their games." Letting them see } \\
\text { there is more, instead of this is it, does not mean more problems, but } \\
\text { allow them to experience a little more of what learning is really about and } \\
\text { to find out in real life how this is being used. They actually will be able to } \\
\text { benefit from this more." }\end{array}$ \\
\hline & & "We are dealing with real life." \\
\hline
\end{tabular}

Figure 24: Inspiring Motivation and Engagement

\section{Incidental Findings: Hopelessness}

The researcher then connected the elements of Critical Hope in relation to the themes that emerged from the teachers' voices. Incidentally, hopelessness emerged and was coded similarly, as in language and behavior of hopelessness, the instructional delivery of hopelessness, the lesson planning of hopelessness, and the paradigm of hopelessness. Figure 25 presents the second coding of Manifestations of Hopelessness in Teachers' Paradigm and Pedagogy. 


\section{Findings: "We find the why of both positions: the}

hopeful and the hopeless one" [Freie, 1994, p.3.).

\begin{tabular}{|c|c|c|c|c|}
\hline & Indicator & Evidence & Themes & Elements \\
\hline & Paradigm of hopelessness & 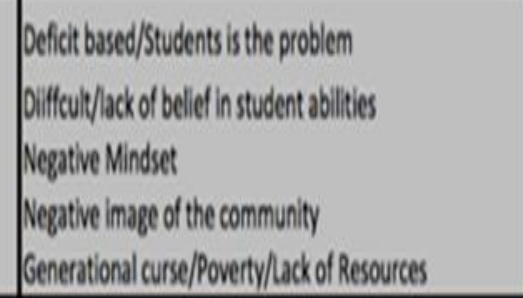 & 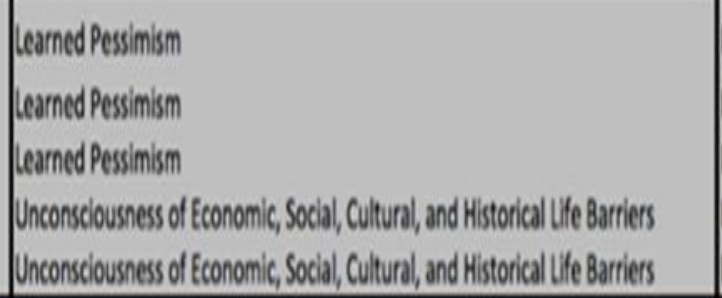 & 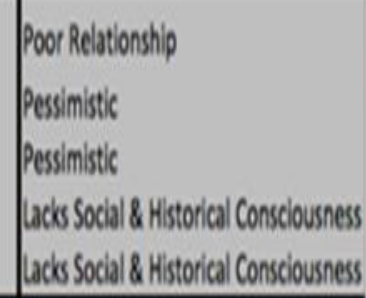 \\
\hline & Instructiona Dellivery of Hopelessness & 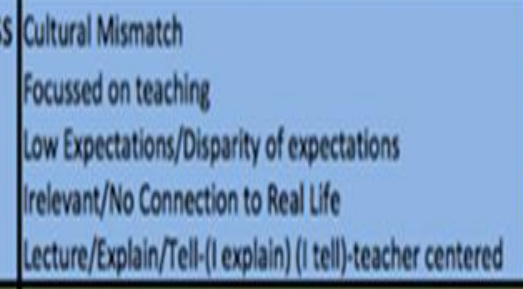 & 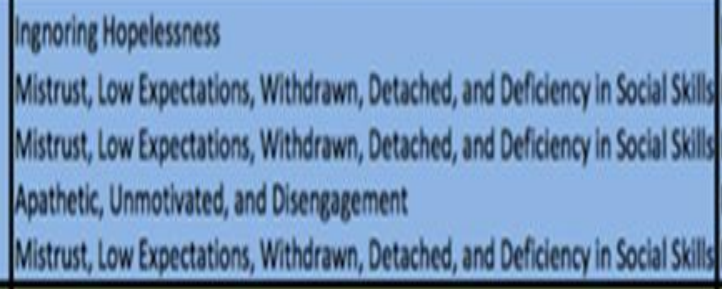 & 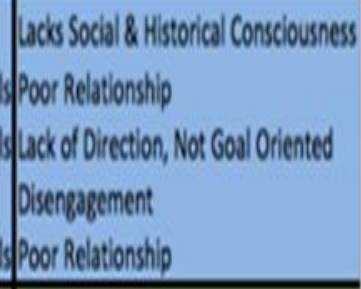 \\
\hline & Instructional Planning of Hopelessnes & 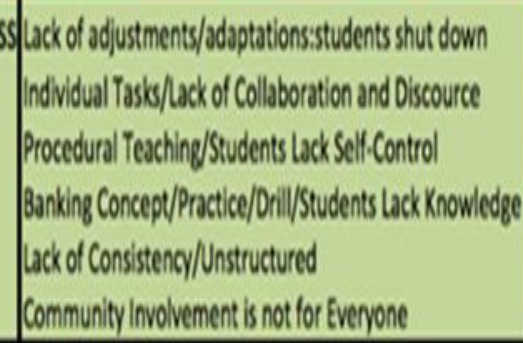 & 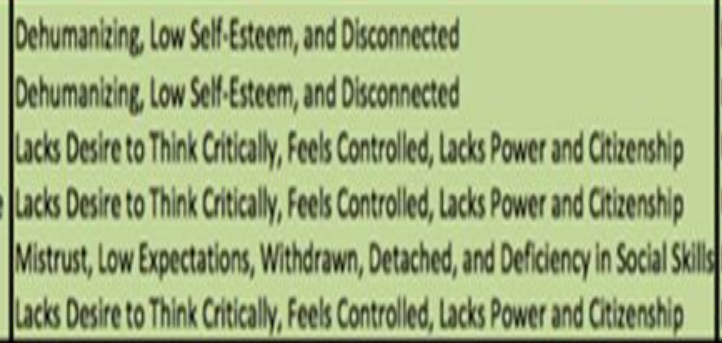 & 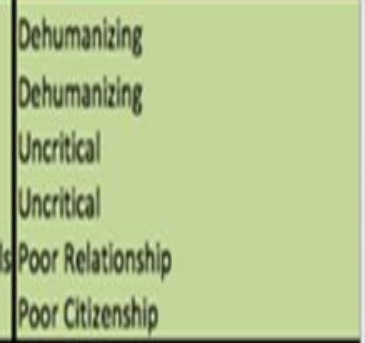 \\
\hline
\end{tabular}




\begin{tabular}{|c|c|c|c|}
\hline Language \& Behavior of Hopelessness & 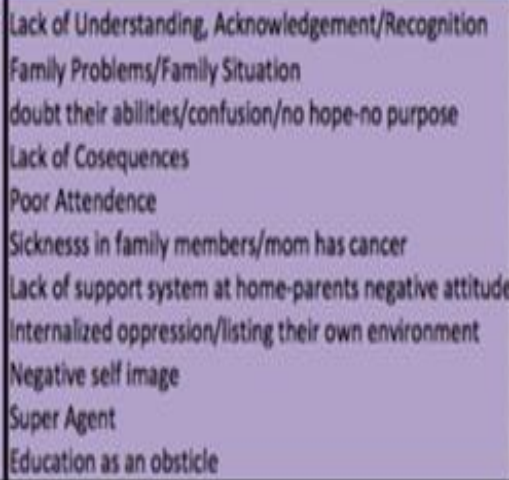 & 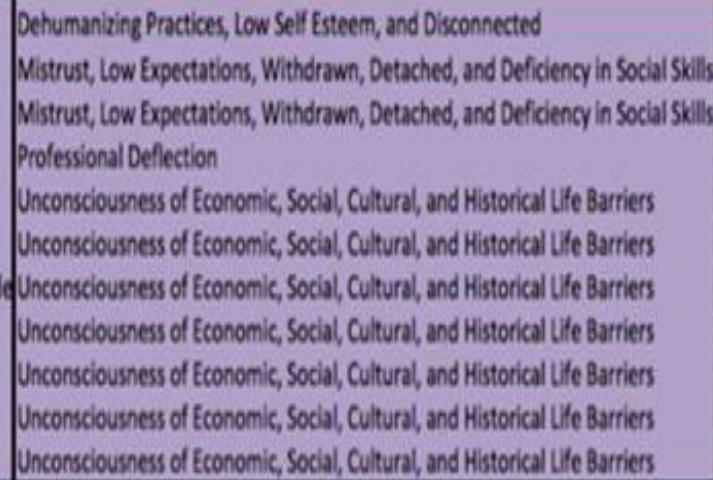 & 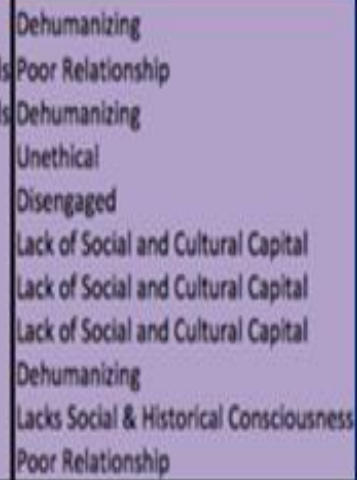 \\
\hline
\end{tabular}

Figure 25: Manifestations of Hopelessness in Teachers' Paradigm and Pedagogy 
In the second hand coding, the researcher coded all 10 transcripts pertaining to hopelessness as in the language and behavior of hopelessness, the instructional delivery of hopelessness, the lesson planning of hopelessness, and the paradigm of hopelessness. Then the researcher identified nine themes pertaining to hopelessness as they emerged from the authentic voices of the teachers and are consistent with the shared experiences of the teachers' in the lowincome middle school.

1. Not Goal Oriented, Depleted Willpower, and Deficiency in Coping

2. Mistrusts, Low Expectations, and Deficiency in Social Skills

3. Uncritical, Feels controlled by Outside Forces, Lacks Power, and Citizenship Skills

4. Learned Pessimism

5. Ignoring Hopelessness

6. Professional Deflection and Lacks Ethical Accountability

7. Dehumanizing self-identity and sense of community

8. Unconsciousness of economic, social, cultural, and historical life barriers

9. Apathetic, Unmotivated, and Disengagement

Using the nine themes of hopelessness, the researcher created NVivo themes and coded all 10 interview transcripts. The researcher then read through all the transcriptions again and hand coded the transcripts looking for the textual evidence from the teachers' voices as it emerged in each of the nine themes hopelessness. 


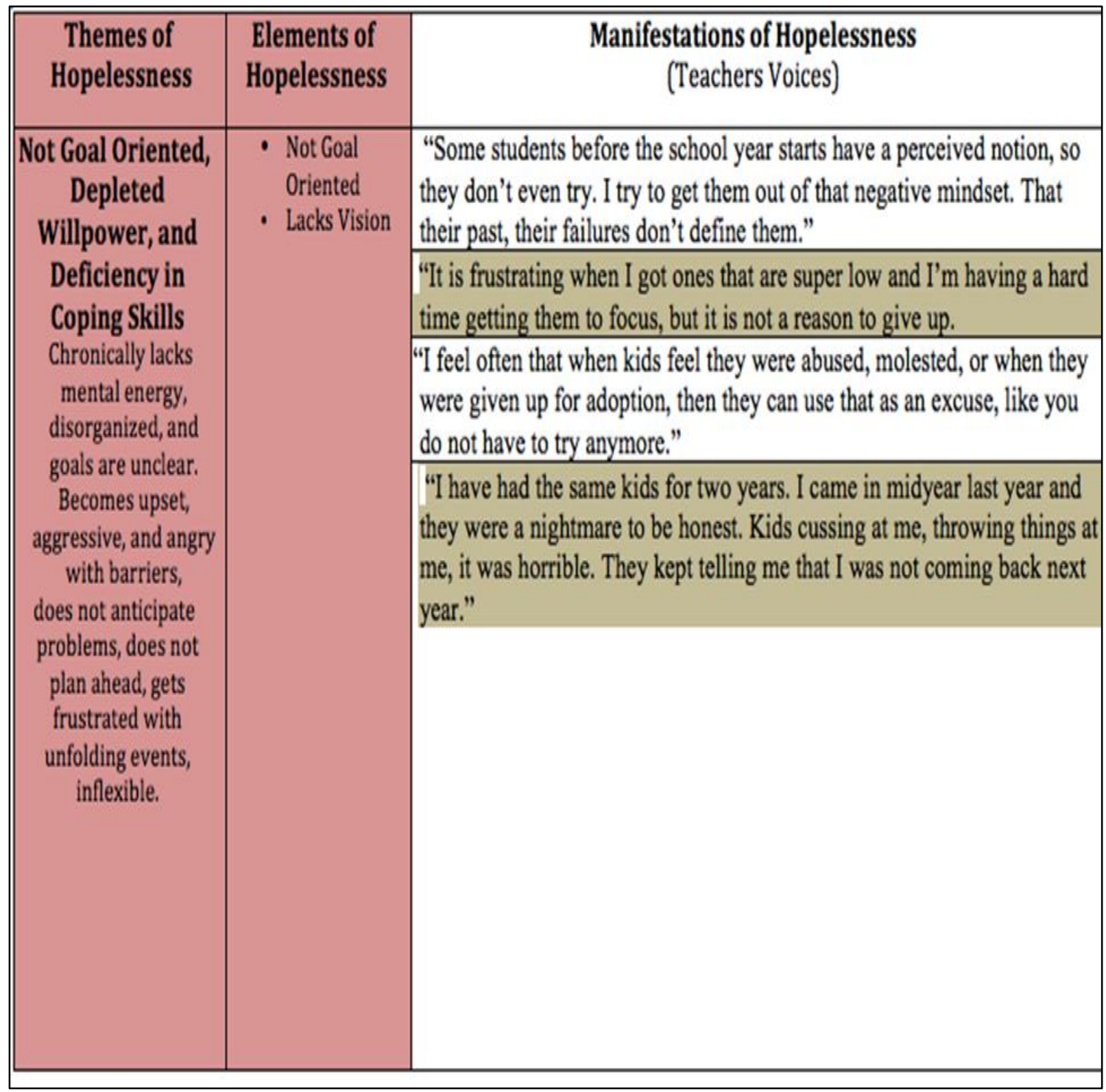

Figure 26: Not Goal Oriented, Depleted Willpower, and Deficiency in Coping 


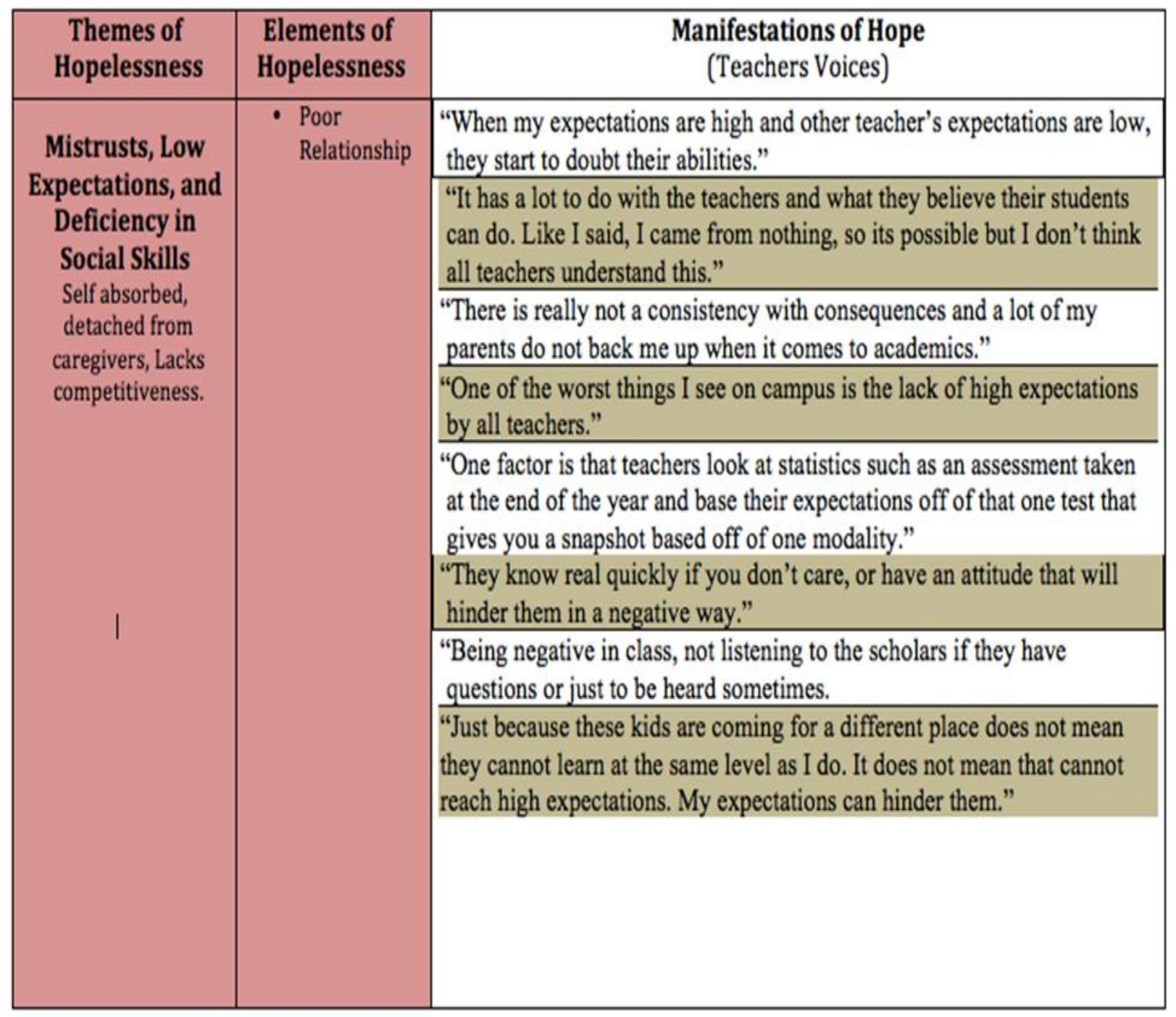

Figure 27: Mistrusts, Low Expectations, and Deficiency in Social Skills 


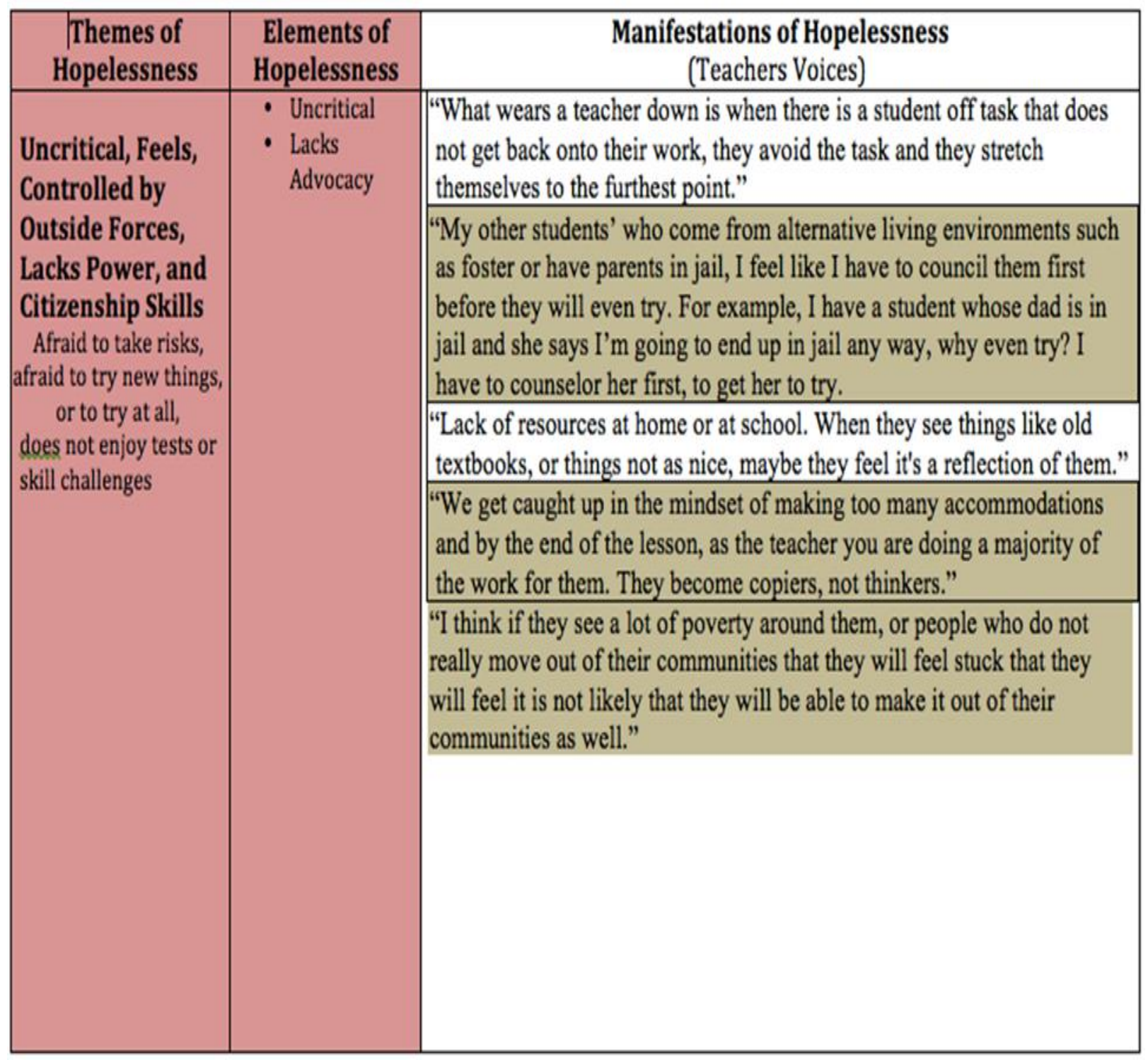

Figure 28: Uncritical, Feels Controlled by Outside Forces, Lacks Power, and Citizenship Skills 


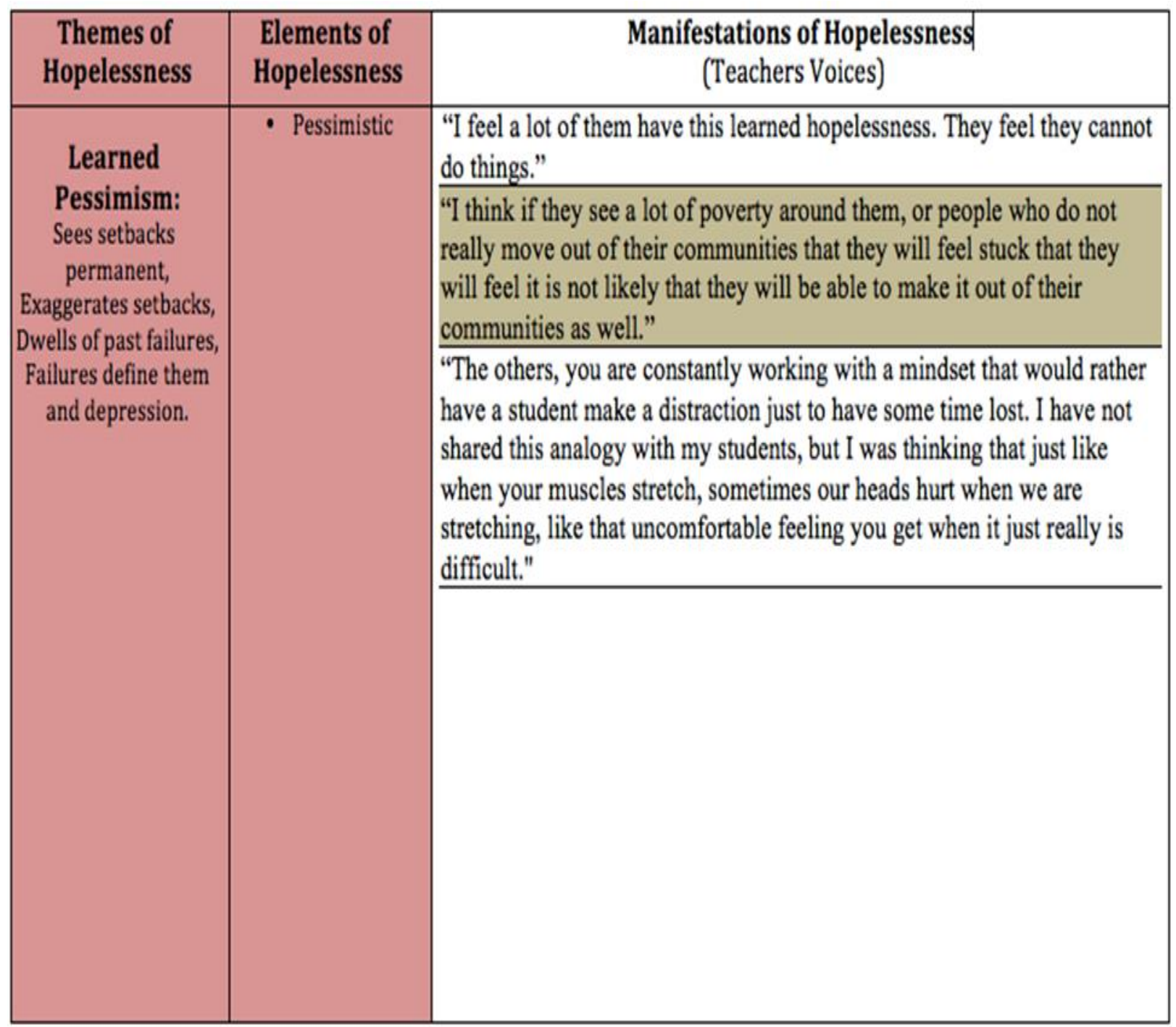

Figure 29: Learned Pessimism 


\begin{tabular}{|c|c|c|}
\hline $\begin{array}{c}\text { Themes of } \\
\text { Hopelessness }\end{array}$ & $\begin{array}{l}\text { Elements of } \\
\text { Hopelessness }\end{array}$ & $\begin{array}{l}\text { Manifestations of Hopelessness } \\
\text { (Teachers Voices) }\end{array}$ \\
\hline $\begin{array}{c}\text { Ignoring } \\
\text { Hopelessness } \\
\text { Through lack of } \\
\text { acknowledgement } \\
\text { and relevancy of their } \\
\text { personal experiences, } \\
\text { lack of belief in their } \\
\text { capabilities, and lack } \\
\text { of understanding. }\end{array}$ & $\begin{array}{l}\text { - Lacks Social } \\
\text { and Historical } \\
\text { Consciousness }\end{array}$ & $\begin{array}{l}\text { "The teacher... And I'll clarify that. The teacher determines those. I think } \\
\text { every teacher on campus understands that the students are special because } \\
\text { of their circumstances, but I do not think that they know how to deal with } \\
\text { those circumstances. Instead of acknowledging that, they talk past it, they } \\
\text { don't speak to it. Instead of using it as motivation as to why they are going } \\
\text { to be better. Maybe because the teachers did not go through it, or no one in } \\
\text { their circle or their life has had to deal with such disadvantages." } \\
\text { "It is easier to teach a book to them, instead of thinking, speaking to them } \\
\text { as if they are people. In addition, it is hard to understand that these } \\
\text { children are dealing with adult circumstances, so they are just children to } \\
\text { them because that is what you see." } \\
\text { "I think many of our students think they are going to be sports figures. I } \\
\text { had a student telling me all about their } 20 \text { some lst place trophies as he } \\
\text { was messing around today. } \\
\text { "I think we confuse our kids with their behavior sometimes. Behaviors can } \\
\text { be outrageous and personally offensive, but at the end of the day they are } \\
\text { an expression of a need that is not being met. If you can connect it to that, } \\
\text { you can move passed the behavior and into the person. If you can't, that } \\
\text { person is the behavior and they are the problem." } \\
\text { "You can't really read It from a book and when they do its general its not } \\
\text { my life." }\end{array}$ \\
\hline
\end{tabular}

Figure 30: Ignoring Hopelessness 


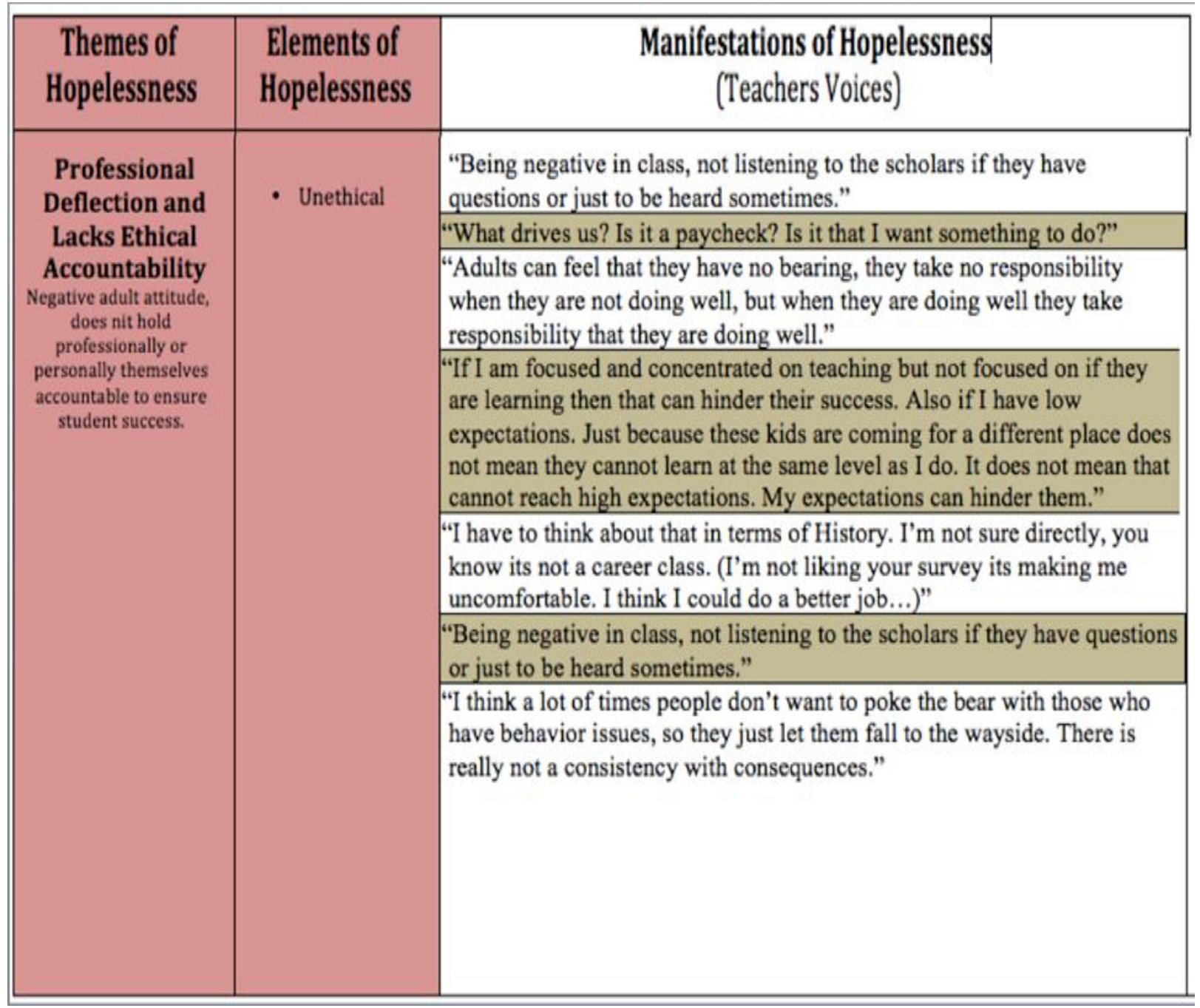

Figure 31: Professional Deflection and Lacks Ethical Accountability 


\begin{tabular}{|c|c|c|}
\hline $\begin{array}{l}\text { Themes of } \\
\text { Hopelessness }\end{array}$ & $\begin{array}{l}\text { Elements of } \\
\text { Hopelessness }\end{array}$ & $\begin{array}{l}\text { Manifestations of Hopelessness } \\
\text { (Teachers Voices) }\end{array}$ \\
\hline \multirow{9}{*}{$\begin{array}{c}\text { Dehumanizing, } \\
\text { Low Self-Esteem, } \\
\text { and } \\
\text { Disconnected: } \\
\text { as a result of chronic } \\
\text { deficiency in } \\
\text { pathways and agency, } \\
\text { incapable of obtaining } \\
\text { past goals }\end{array}$} & \multirow[t]{9}{*}{ - Dehumanizing } & $\begin{array}{l}\text { "I am realizing that our school community struggles with their self-image, } \\
\text { a lot of what I do is targeted at making students feel that they are capable } \\
\text { of reaching their goals." } \\
\text { "I think sometimes their hope is in the wrong places." }\end{array}$ \\
\hline & & $\begin{array}{l}\text { "If they feel they are the ones that are bad, instead of their behavior that } \\
\text { is a poor choice, then that will hinder their aspirations." }\end{array}$ \\
\hline & & $\begin{array}{l}\text { "I read about something called stereotype threat, which is not other } \\
\text { people stereotyping, but its themselves, how they see themselves that is } \\
\text { limiting them from reaching their potential." }\end{array}$ \\
\hline & & $\begin{array}{l}\text { "Being negative in class, not listening to the scholars if they have } \\
\text { questions or just to be heard sometimes." }\end{array}$ \\
\hline & & $\begin{array}{l}\text { "I needed a teacher to really see me and not just look at a class of } 35, \text { but } \\
\text { really see me. }\end{array}$ \\
\hline & & $\begin{array}{l}\text { "One factor is that teachers look at statistics such as an assessment taken } \\
\text { at the end of the year and base their expectations off of that one test that } \\
\text { gives you a snapshot based off of one modality." }\end{array}$ \\
\hline & & $\begin{array}{l}\text { "I think we confuse our kids with their } \\
\text { behavior sometimes." }\end{array}$ \\
\hline & & $\begin{array}{l}\text { "For instance reading scores that have gone several years at the same } \\
\text { level, so it gets brushed under the rug and not addressed." }\end{array}$ \\
\hline & & $\begin{array}{l}\text { "I think many of our students think they are going to be sports figures. I } \\
\text { had a student telling me all about their } 20 \text { some } 1 \text { st place trophies as he } \\
\text { was messing around today." }\end{array}$ \\
\hline & & \\
\hline
\end{tabular}

Figure 32: Dehumanizing Self-identity and Sense of Community 


\begin{tabular}{|c|c|c|}
\hline $\begin{array}{l}\text { Themes of } \\
\text { Hopelessness }\end{array}$ & $\begin{array}{l}\text { Elements of } \\
\text { Hopelessness }\end{array}$ & $\begin{array}{l}\text { Manifestations of Hopelessness } \\
\text { (Teachers Voices) }\end{array}$ \\
\hline \multirow{7}{*}{$\begin{array}{l}\text { Unconscious of } \\
\text { Economic, Social, } \\
\text { Cultural, and } \\
\text { Historical Life } \\
\text { Barriers } \\
\text { Lack of awareness of } \\
\text { systemic oppression } \\
\text { and personal barriers } \\
\text { in students 'daily lives. }\end{array}$} & \multirow{7}{*}{$\begin{array}{l}\text { Lack of Social } \\
\text { and Historical } \\
\text { Consciousness } \\
\text { - Lack of } \\
\text { Knowledge of } \\
\text { Social and } \\
\text { Cultural } \\
\text { Capital }\end{array}$} & $\begin{array}{l}\text { "There are sometimes things in life that you cannot change. I am aware } \\
\text { that there are things that are pulling at them. Sometimes they are willing } \\
\text { to share and sometimes they are not." }\end{array}$ \\
\hline & & $\begin{array}{l}\text { "Politics, some of my students are so entrenched in their own } \\
\text { neighborhood and their own culture that they do not really understand } \\
\text { what is going on around them." }\end{array}$ \\
\hline & & $\begin{array}{l}\text { "I think if they see a lot of poverty around them, or people who do not } \\
\text { really move out of their communities that they will feel stuck that they } \\
\text { will feel it is not likely that they will be able to make it out of their } \\
\text { communities as well." }\end{array}$ \\
\hline & & $\begin{array}{l}\text { "I think community involvement can help you achievement high places in } \\
\text { life. I am not sure that everyone is cut out for community involvement." }\end{array}$ \\
\hline & & $\begin{array}{l}\text { "I ask, 'Where do you want to live?' Most of our scholars say Rancho } \\
\text { Cucamonga. They say because its near the airport and I want to travel. I } \\
\text { don't have to worry about gunshots at night because there are no gangs. } \\
\text { They start listing and what they are really doing is listing their own } \\
\text { environment." }\end{array}$ \\
\hline & & $\begin{array}{l}\text { "For example, one time my administrator said to me what my people } \\
\text { signify in the status quo of culture and my community." }\end{array}$ \\
\hline & & $\begin{array}{l}\text { "Here they are living that same generational curse, their parents did not } \\
\text { make it out, and yet, they are the ones expected to break the cycle." }\end{array}$ \\
\hline
\end{tabular}

Figure 33: Unconsciousness of Economic, Social, Cultural, and Historical Life Barriers 


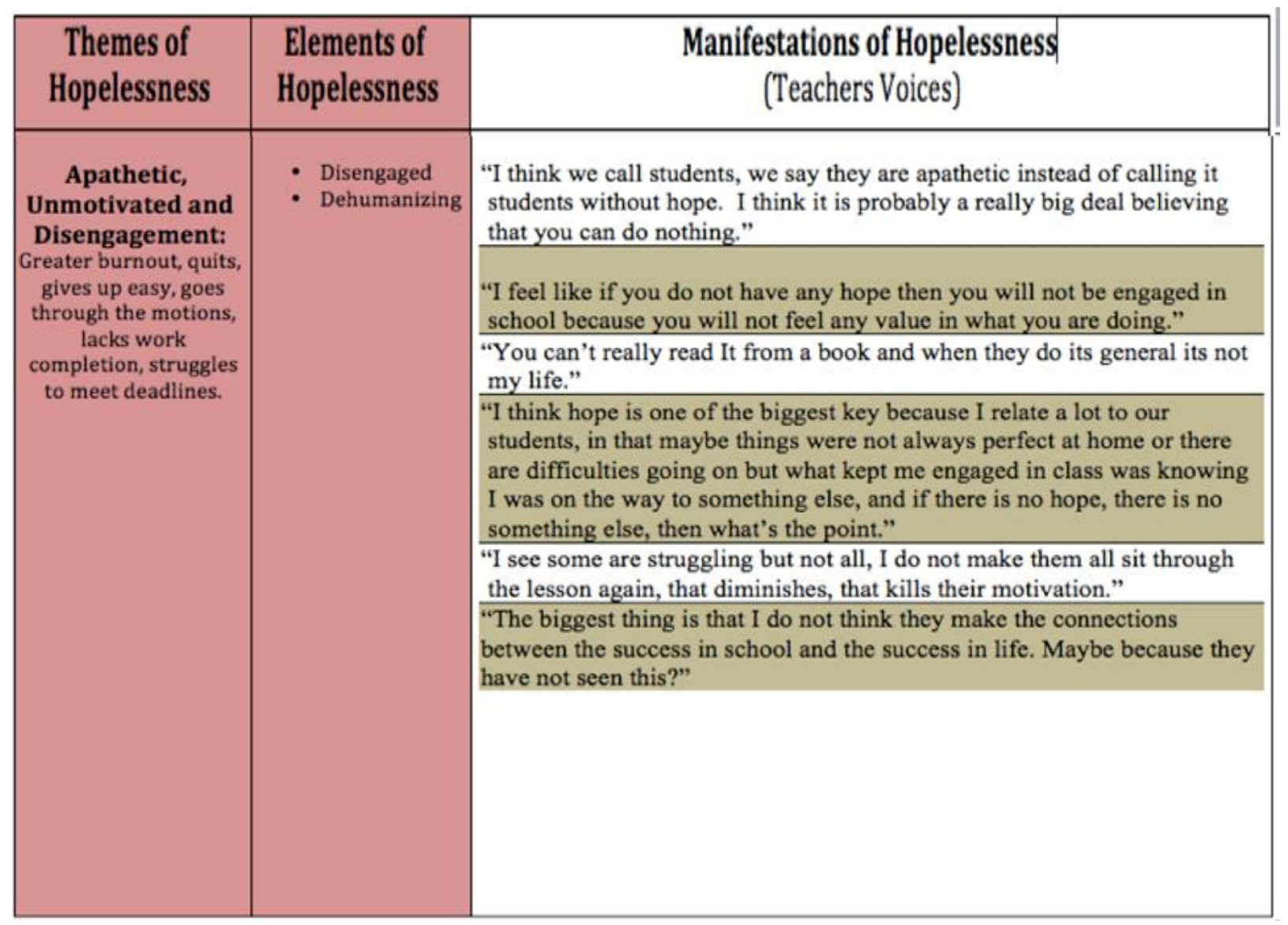

Figure 34: Apathetic, Unmotivated, and Disengaged

\section{Incidental Findings: Unintentional Hopelessness}

Many people in society do not acknowledge that everyday practices in schools often comply with or contribute to hopelessness and oppression through traditional and historic ways, cultural myths, and dominating views and practices of common teachers. In order to create change, people who see this must challenge the common sense of what teachers do and how they think (Kumashiro, 2015). Now I do not believe that educators are purposely oppressing and intentionally contributing to hopelessness in their students, but rather, I believe that this is a result in a lack of awareness and understanding of who their students are what their life 
experiences entail, and what is needed to increase their learning and facilitate their engagement (Lalas et al., 2016). Subsequently, other incidental findings revealed the danger and troubling ways common teaching practices unintentionally contribute to hopelessness through oppressive acts of symbolic violence, misrecognition, unstable and oppressive environments, self-deception, self-depreciation, and narrative sickness. Thus, we need a paradigm shift representing an education of Critical Hope, as hope is an ontological need and we must take care not to unintentionally allow it to slip toward hopelessness and despair (Freire, 1994; see Figure 35).

In the third hand coding, the researcher coded all 10 transcripts pertaining to unintentional hopelessness created by teachers through language and behavior, the instructional delivery, and the lesson planning, which were all driven by their paradigm of hopelessness. In Figure 36, the six categories of unintentional hopelessness with its descriptor are listed in one column with the textual evidence from the teachers' voices that support that category.

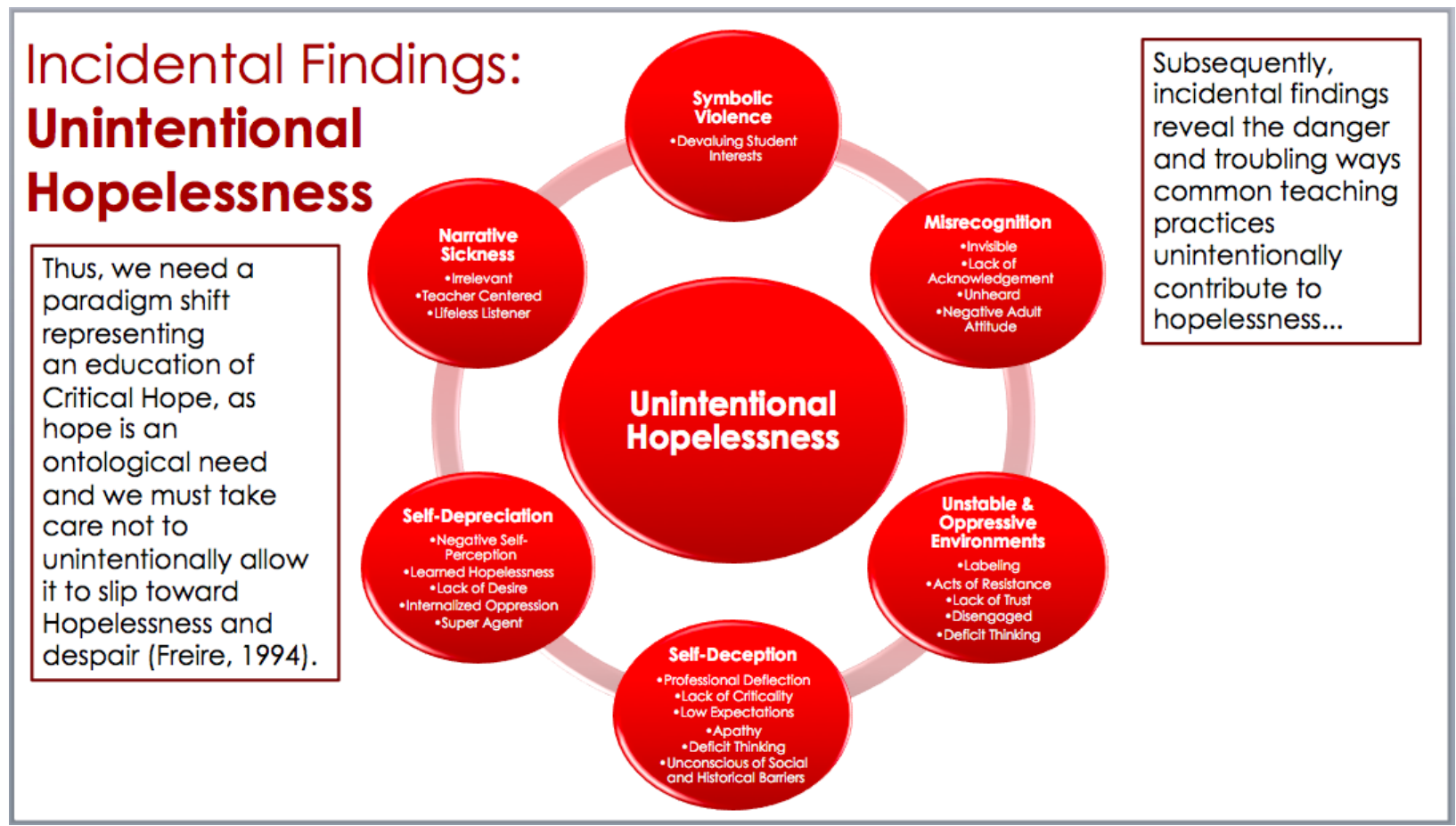

Figure 35: Incidental Findings Unintentional Hopelessness 
EXAMINING TEACHERS' PARADIGM AND PEDAGOGY IN MANIFESTING CRITICAL HOPE IN LOW-INCOME MIDDLE SCHOOL

\begin{tabular}{|c|c|c|}
\hline $\begin{array}{l}\text { Unintentional Hopelessness } \\
\text { Themes }\end{array}$ & Indicators & Textual Evidence \\
\hline $\begin{array}{l}\text { Symbolic Violence } \\
\text { "The experience of having one's culture, } \\
\text { knowledge Language, Tastes, and } \\
\text { dispositions devalued" (Saltman, 2018, p.6). }\end{array}$ & $\begin{array}{l}\text { - Devaluing } \\
\text { Student Interests }\end{array}$ & $\begin{array}{l}\text { "I think many of our students think they are going to be sports figures. I had a student telling me all about } \\
\text { their } 20 \text { some Ist place trophies as he was messing around today. I tried to make some connections for } \\
\text { him. I think sometimes their hope is in the wrong places. So maybe it isn't teven that they are not hopeful, } \\
\text { but that their hope is in the wrong places. }\end{array}$ \\
\hline \multirow{3}{*}{$\begin{array}{l}\text { Misrecognition/Lack of recognition } \\
\text { "Nonrecegnition or misrecognition... can be } \\
\text { a form of oppression, imprisoning someone } \\
\text { in a false, distorted, reduced mode of being. } \\
\text { Beyond simple lack of respect, it can inflict a } \\
\text { grievous wound, saddling people with } \\
\text { crippling self-hatred. Due recognition is not } \\
\text { just a courtesy but a vital human need. } \\
\text { (Frazer, 1997p. 25; Taylor, 1992). }\end{array}$} & \multirow{3}{*}{$\begin{array}{l}\text { - Invisible } \\
\text { - Lack of } \\
\text { Acknowledgement } \\
\text { - Not Listening } \\
\text { - Negative } \\
\text { Attitude }\end{array}$} & "I needed a teacher to really see me and not just look at a class of 35 , but really see me." \\
\hline & & $\begin{array}{l}\text { "The teacher... And I'll clarify that. The teacher determines those. I think every teacher on campus } \\
\text { understands that the students are special because of their circumstances, but I do not think that they know } \\
\text { how to deal with those circumstances. Instead of acknowledging that, they talk past it, they don't speak to } \\
\text { it. Instead of using it as motivation as to why they are going to be better. Maybe because the teachers did } \\
\text { not go through it, or no one in their circle or their life has had to deal with such disadvantages. It is easier } \\
\text { to teach a book to them, instead of thinking and speaking to them as if they are people. In addition, it is } \\
\text { hard to understand that these children are dealing with adult circumstances, so they are just children to } \\
\text { them because that is what you see." }\end{array}$ \\
\hline & & $\begin{array}{l}\text { "Being negative in class, not listening to the scholars if they have questions or just to be heard sometimes. } \\
\text { They know real quickly if you don't care, or have an attitude that will hinder them in a negative way. }\end{array}$ \\
\hline \multirow{5}{*}{$\begin{array}{l}\text { Unstable and 0ppressive } \\
\text { Environments } \\
\text { Dehumanizing leads the oppressed to } \\
\text { struggle against their oppressors } \\
\text { (Freire, 1970) }\end{array}$} & \multirow{5}{*}{$\begin{array}{l}\text { - Labeling } \\
\text { Students } \\
\text { - Acts of } \\
\text { Resistance } \\
\text { - Lack of Trust } \\
\text { - Disengaged } \\
\text { - Deficit } \\
\text { Thinking }\end{array}$} & $\begin{array}{l}\text { "I think we confuse our kids with their behavior sometimes. Behaviors can be outrageous and personally } \\
\text { offensive, but at the end of the day they are an expression of a need that is not being met. If you can } \\
\text { connect it to that, you can move passed the behavior and into the person. And if you can't that person is } \\
\text { the behavior and they are the problem." }\end{array}$ \\
\hline & & $\begin{array}{l}\text { "Its funny a student that tried to fight me last year says now I'm her favorite teacher. It took a while for } \\
\text { them to trust me to accept me. They came in at the beginning of the year and they said you're not mad at } \\
\text { me and I said no. The big thing was they said your not coming back and then I did." }\end{array}$ \\
\hline & & $\begin{array}{l}\text { "It is frustrating when I got ones that are super low and I'm having a hard time getting them to focus, but } \\
\text { it is not a reason to give up. What wears a teacher down is when there is a student off task that does not } \\
\text { get back onto their work, they avoid the task and they stretch themselves to the furthest point." }\end{array}$ \\
\hline & & $\begin{array}{l}\text { "I have had the same kids for two years. I came in midyear last year and they were a nightmare to be } \\
\text { honest. Kids cussing at me, throwing things at me, it was horrible. They kept telling me that I was not } \\
\text { coming back next year. Then summer came and they saw me. They came in and were able to follow the } \\
\text { routine and are a lot more kind with one another now. Kids that snapped at each other are now kind to } \\
\text { each other and are helping each other to learn how to read." }\end{array}$ \\
\hline & & $\begin{array}{l}\text { "The hopeful students are working and completing tasks. The others, you are constantly working with a } \\
\text { mindset that would rather have a student make a distraction just to have some time lost. I have not shared } \\
\text { this analogy with my students, but I was thinking that just like when your muscles stretch, sometimes our } \\
\text { heads hurt when we are stretching, like that uncomfortable feeling you get when it just really is difficult." }\end{array}$ \\
\hline
\end{tabular}

Figure 36: Unintentional Hopelessness Symbolic Violence, Misrecognition, and Unstable Environments 
EXAMINING TEACHERS' PARADIGM AND PEDAGOGY IN MANIFESTING CRITICAL HOPE IN LOW-INCOME MIDDLE SCHOOL

Symbolic Violence is "The experience of having one's culture, knowledge language, tastes, and dispositions devalued" (Saltman, 2018, p. 6). This was demonstrated in the devaluing of student's interests. For example, Participant 9 stated,

I think many of our students think they are going to be sports figures. I had a student telling me all about their 20 some 1 st place trophies as he was messing around today. I tried to make some connections for him. I think sometimes their hope is in the wrong places. So maybe it isn't even that they are not hopeful, but that their hope is in the wrong places.

Misrecognition/Lack of recognition: Charles Taylor (1992 as cited in Fraser, 1997) argued:

Nonrecognition or misrecognition ... can be a form of oppression, imprisoning someone in a false, distorted, reduced mode of being. Beyond simple lack of respect, it can inflict a grievous wound, saddling people with crippling self-hatred. Due recognition is not just a courtesy but a vital human need. (p. 14).

This is when students seem invisible because there is a Lack of Acknowledgement of them, not listening to the students, teachers with negative, adult attitude. For example, Participant 5 stated, The teacher., . . And I'll clarify that. The teacher determines those. I think every teacher on campus understands that the students are special because of their circumstances, but I do not think that they know how to deal with those circumstances. Instead of acknowledging that, they talk past it, they don't speak to it, instead of using it as motivation as to why they are going to be better. Maybe because the teachers did not go through it, or no one in their circle or their life has had to deal with such disadvantages. It is easier to teach a book to them, instead of thinking and speaking to 
EXAMINING TEACHERS' PARADIGM AND PEDAGOGY IN MANIFESTING CRITICAL HOPE IN LOW-INCOME MIDDLE SCHOOL

them as if they are people. In addition, it is hard to understand that these children are dealing with adult circumstances, so they are just children to them because that is what you see.

Unstable and Oppressive Environments: Can be observed by labeling students in a negative manner such as problem students, behavioral students, and low achievers. Teachers noted what I call acts of resistance by students who display negative behaviors. There is a lack of trust from teacher to student, which causes students to exhibit Disengagement behaviors. Followed by teachers with deficit thinking about what their students can do to achieve. For example, Participant 9 stated,

The hopeful students are working and completing tasks. The others, you are constantly working with a mindset that would rather have a student make a distraction just to have some time lost. I have not shared this analogy with my students, but I was thinking that just like when your muscles stretch, sometimes our heads hurt when we are stretching, like that uncomfortable feeling you get when it just really is difficult.

Self Deception occurs when teachers display a lack of confidence in their students' abilities, in what they think they should already know and think by middles school. This leads teachers to use self-deflection and not take responsibility for students' learning but rather they blame shift, it's the students as having a lack of motivation, the parents, or the teacher-from-theprior-years' fault. As a result, they have low expectations for student achievement, they have deficit thinking in students' abilities, and they are unaware of the social and historical life barriers. For example, Participant 1 stated, "One of the worst things I see on campus is the lack of high expectations from all teachers." 
Self-Depreciation is when students internalize these negative hopeless behaviors and they become convinced of their unfitness toward success. For example, Participant 10 stated, "I feel a lot of them have this learned hopelessness. They feel they cannot do things."

Finally, Narrative Sickness is when teachers do all the talking and students sit passively and idly by coping and listening to the teacher. The lectures are irrelevant, teacher centered, and students are lifeless inside the classroom. For example, Participant 3 was discussing irrelevant content and stated, "You can't really read it from a book and when they do it's general, it's not my life." 


\begin{tabular}{|c|c|c|}
\hline \multirow{13}{*}{$\begin{array}{l}\text { Self-Deception } \\
\text { "Lack of confidence in their abilities to } \\
\text { think to want and to know." (Evejice, 1970). }\end{array}$} & \multirow{13}{*}{$\begin{array}{l}\text { - Professional } \\
\text { Deflection } \\
\text { - Lack of } \\
\text { Criticality } \\
\text { - Low } \\
\text { expectations } \\
\text { - Apathy } \\
\text { - Deficit } \\
\text { Thinking } \\
\text { - Unconscious } \\
\text { of Social and } \\
\text { Historical } \\
\text { Barriers }\end{array}$} & $\begin{array}{l}\text { "For instance reading scores that have gone several years at the same level, so it gets brushed under the } \\
\text { rug and not addressed." }\end{array}$ \\
\hline & & $\begin{array}{l}\text { "There are also some things I do have control over. One is adult attitude. Adults can feel that they have no } \\
\text { bearing, they take no responsibility when they are not doing well, but when they are doing well they take } \\
\text { responsibility that they are doing well. }\end{array}$ \\
\hline & & "I have to think about that in terms of History. I'm not sure directly, you know its not a career class." \\
\hline & & $\begin{array}{l}\text { "I think a lot of times people don't want to poke the bear with those who have behavior issues, so they just } \\
\text { let them fall to the wayside. There is really not a consistency with consequences." }\end{array}$ \\
\hline & & $\begin{array}{l}\text { "We get caught up in the mindset of making too many accommodations and by the end of the lesson, as } \\
\text { the teacher you are doing a majority of the work for them. They become copiers, not thinkers." }\end{array}$ \\
\hline & & $\begin{array}{l}\text { "When my expectations are high and other teacher's expectations are low, they start to doubt their } \\
\text { abilities." }\end{array}$ \\
\hline & & "One of the worst things I see on campus is the lack of high expectations by all teachers." \\
\hline & & $\begin{array}{l}\text { "It has a lot to do with the teachers and what they believe their students can do. Like I said, I came from } \\
\text { nothing, so its possible but I don't think all teachers understand this." }\end{array}$ \\
\hline & & $\begin{array}{l}\text { "Also if I have low expectations. Just because these kids are coming for a different place does not mean } \\
\text { they cannot learn at the same level as I do. It does not mean that cannot reach high expectations. My } \\
\text { expectations can hinder them." }\end{array}$ \\
\hline & & $\begin{array}{l}\text { "One factor is that teachers look at statistics such as an assessment taken at the end of the year and base } \\
\text { their expectations off of that one test that gives you a snapshot based off of one modality." }\end{array}$ \\
\hline & & $\begin{array}{l}\text { "I think we call students, we say they are apathetic instead of calling it students without hope. I think it is } \\
\text { probably a really big deal believing that you can do nothing." }\end{array}$ \\
\hline & & $\begin{array}{l}\text { "I think community involvement can help you achievement high places in life. I am not sure that everyone } \\
\text { is cut out for" community involvement." }\end{array}$ \\
\hline & & $\begin{array}{l}\text { "As much as I want them to keep their innocence and their culture, they also have to know they have to } \\
\text { have a voice because someone is going to try to burst your bubble someday. You must know how to } \\
\text { respond in these situations. If you don't tell them, they will go out and set themselves up for failuere and get } \\
\text { knocked off of their' journey. It will take them a while to come back. They need to know how others see } \\
\text { what your place is in society and how you have to work against that place, and if no one ever tells you, } \\
\text { you do not know what you are working against." }\end{array}$ \\
\hline
\end{tabular}

Figure 37: Unintentional Hopelessness Self Deception 
EXAMINING TEACHERS' PARADIGM AND PEDAGOGY IN MANIFESTING CRITICAL HOPE IN LOW-INCOME MIDDLE SCHOOL

\begin{tabular}{|c|c|c|}
\hline \multirow{6}{*}{$\begin{array}{l}\text { Self-Depreciation } \\
\text { "In the end they become convinced of their } \\
\text { own unfitness" (Ereize, 1970). }\end{array}$} & \multirow{6}{*}{$\begin{array}{|ll|}\text { - } & \text { Negative Self- } \\
\text { Perception } \\
\text { - Learned } \\
\text { Hopelessness } \\
\text { - Lack of Desire } \\
\text { - Internalized } \\
\text { Oppression } \\
\text { - Super Agent } \\
\end{array}$} & $\begin{array}{l}\text { "If they feel they are the ones that are bad, instead of their behavior that is a poor choice, then that will } \\
\text { hinder their aspirations." }\end{array}$ \\
\hline & & $\begin{array}{l}\text { "I read about something called stereotype threat, which is not other people stereotyping, but its } \\
\text { themselves, how they see themselves that is limiting them from reaching their potential." }\end{array}$ \\
\hline & & "I feel a lot of them have this learned hopelessness. They feel they cannot do things." \\
\hline & & $\begin{array}{l}\text { "My other students' who come from alternative living environments such as foster or have parents in jail, I } \\
\text { feel like I have to council them first befoore they will even try. For example, I have a student whose dad is } \\
\text { in jail and she says I'm going to end up in jail any way, why even try. I have to counselor her first, to get } \\
\text { her to try." }\end{array}$ \\
\hline & & $\begin{array}{l}\text { "I feel often that when kids feel they were abused, molested, or when they were given up for adoption, } \\
\text { then they can use that as an excuse, like you do not have to try anymore." }\end{array}$ \\
\hline & & $\begin{array}{l}\text { "Their everyday life! Their everyday life hinders their aspirations just the fact that they wake up and are } \\
\text { all sharing one mattress, the fact there is not food in the house, and the fact that they walk this street, } \\
\text { where they see the condoms, the needles, the beer bottles, all of this stuff saying that this is the lower class } \\
\text { people, this is what you will end up being... you will end up drunk, pregnant and on drugs, they walk over } \\
\text { that every day. They come to a classroom here, where multiple people have the same stories. Here they are } \\
\text { living that same generational curse, their parents did not make it out, and yet, they are the ones expected to } \\
\text { break the cycle." }\end{array}$ \\
\hline \multirow{5}{*}{$\begin{array}{l}\text { Narration Sickness } \\
\text { "Education is suffering from narration } \\
\text { sickness where students are lifeless } \\
\text { listeners" (Everive, 1970, p. 67). }\end{array}$} & \multirow{5}{*}{$\begin{array}{l}\text { - Irrelevant } \\
\text { - Teacher } \\
\text { Centered } \\
\text { - Lifeless } \\
\text { Listener }\end{array}$} & "You can't really read It from a book and when they do its general its not my life." \\
\hline & & $\begin{array}{l}\text { "If I am focused and concentrated on teaching but not focused on if they are learning then that can hinder" } \\
\text { their success." }\end{array}$ \\
\hline & & $\begin{array}{l}\text { "The biggest thing is that I do not think they make the connections between the success in school and the } \\
\text { success in life. Maybe because they have not seen this?" }\end{array}$ \\
\hline & & $\begin{array}{l}\text { "Today, I pulled in several students to talk about their potential and their leadership skills, and to } \\
\text { encourage them to use them in a productive way. I told them that they had gitts and they should use those } \\
\text { gitts, and that I did not see them using it for the best right now." }\end{array}$ \\
\hline & & $\begin{array}{l}\text { "I explain to them that what we are doing in the classroom makes them ready for jobs and prepares them } \\
\text { for college, which can help them with the income. I try to make a connection between what we are doing } \\
\text { in here and what they want to do in the future. School gives you choices. The more you do in school, the } \\
\text { more educated you are, the more choices are opened up for you." }\end{array}$ \\
\hline
\end{tabular}

Figure 38: Unintentional Hopelessness Self Depreciation and Narration Sickness 


\section{EXAMINING TEACHERS' PARADIGMAND PEDAGOGY IN MANIFESTING CRITICAL HOPE IN LOW-INCOME MIDDLE SCHOOL}

\section{Hope Gap}

This led to the discovery that a hope gap exists, which I am defining as the zone of difference representing sets of expressions and actions that reflect hope and hopelessness in humanity.

\begin{tabular}{|c|c|c|c|c|}
\hline $\begin{array}{l}\text { Manifestations of Hope } \\
\text { (Teachers Voices) }\end{array}$ & $\begin{array}{l}\text { Themes of } \\
\text { Critical Hope }\end{array}$ & $\begin{array}{l}\text { Hope } \\
\text { Gap }\end{array}$ & $\begin{array}{l}\text { Themes of } \\
\text { Hopelessness }\end{array}$ & $\begin{array}{l}\text { Manifestations of Hopelessness } \\
\text { (Teachers Voices) }\end{array}$ \\
\hline $\begin{array}{l}\text { "The hopeful students are working and completing } \\
\text { tasks." }\end{array}$ & \multirow{14}{*}{\begin{tabular}{|} 
Goal Oriented, \\
Willingness to \\
Act, and \\
Perseverance \\
Mentally invested, \\
engaged, focused \\
on achieving clear \\
and difficult goals. \\
Embraces barriers \\
and sees them as a \\
challenge. \\
Anticipates \\
problems, plans \\
ahead for \\
roadblocks, has \\
patience with \\
unfolding events \\
and is mentally \\
flexible. \\
ELEMENTS \\
-Agency \\
-Caring \\
Relationships \\
-Goal Directed \\
Thinking \\
-Navigating \\
Pathways \\
-Optimism \\
-Social and \\
Cultural Capital
\end{tabular}} & \multirow{8}{*}{\begin{tabular}{|c|}
$\mid$ \\
Zone of \\
Difference
\end{tabular}} & \multirow{8}{*}{\begin{tabular}{|c|} 
Not Goal \\
Oriented, \\
Depleted \\
Willpower, \\
and \\
Deficiency in \\
Coping Skills \\
Chronically \\
lacks mental \\
energy, \\
disorganized, \\
and goals are
\end{tabular}} & \multirow{3}{*}{$\begin{array}{l}\text { "Some students before the school year starts } \\
\text { have a perceived notion, so they don't even } \\
\text { try. I try to get them out of that negative } \\
\text { mindset. That their past, their failures don't } \\
\text { define them." }\end{array}$} \\
\hline "I build a culture of having a goal." & & & & \\
\hline \multirow{2}{*}{$\begin{array}{l}\text { "We talk a lot with our kids about goal setting, their } \\
\text { future careers, their strengths, and what they are } \\
\text { good at." }\end{array}$} & & & & \\
\hline & & & & \multirow{5}{*}{$\begin{array}{l}\text { "It is frustrating when I got ones that are } \\
\text { super low and I'm having a hard time getting } \\
\text { them to focus, but it is not a reason to give } \\
\text { up. } \\
\text { "I feel often that when kids feel they were } \\
\text { abused, molested, or when they were given } \\
\text { up for adoption, then they can use that as an } \\
\text { excuse, like you do not have to try anymore." }\end{array}$} \\
\hline $\begin{array}{l}\text { "With goal setting, with career day projects, I try to } \\
\text { guide them towards always having something } \\
\text { specific they are working towards. So they }\end{array}$ & & & & \\
\hline & & & & \\
\hline lot about failure in class as a step toward suocess." & & & & \\
\hline "If you have hope you will try." & & & & \\
\hline $\begin{array}{l}\text { "There are many factors that make people } \\
\text { successful like grit, and when we are working } \\
\text { together for a common goal it changes the mindset } \\
\text { like this person believes in me." }\end{array}$ & & \multirow{6}{*}{$\begin{array}{l}\text { The Hope Gap } \\
\text { is the zone of } \\
\text { difference } \\
\text { representing } \\
\text { Sets of } \\
\text { Expressions } \\
\text { and actions } \\
\text { that reflect } \\
\text { hope and } \\
\text { hopelessness } \\
\text { in humanity. }\end{array}$} & $\begin{array}{l}\text { unclear. } \\
\text { Becomes } \\
\text { upset, } \\
\text { aggressive, and }\end{array}$ & \multirow{6}{*}{$\begin{array}{l}\text { "I have had the same kids for two years. I } \\
\text { came in midyear last year and they were a } \\
\text { nightmare to be honest. Kids cussing at me, } \\
\text { throwing things at me, it was horrible. They } \\
\text { kept telling me that I was not coming back } \\
\text { next year." }\end{array}$} \\
\hline $\begin{array}{l}\text { "So I believe that if kids see that they can be } \\
\text { successful then they are more likely to try to be } \\
\text { successful." }\end{array}$ & & & $\begin{array}{l}\text { angry with } \\
\text { barriers, } \\
\text { does not }\end{array}$ & \\
\hline $\begin{array}{l}\text { "You have to have plans and plans start now. There } \\
\text { has to be a work ethic to go with it. You might not } \\
\text { want to go to college but it is work ethnic that you } \\
\text { need to be successful for your future." }\end{array}$ & & & $\begin{array}{l}\text { anticipate } \\
\text { problems, does } \\
\text { not plan ahead, } \\
\text { gets frustrated }\end{array}$ & \\
\hline $\begin{array}{l}\text { "We talk a lot with our kids about goal setting, } \\
\text { their future careers, their strengths, and what they } \\
\text { are good at." }\end{array}$ & & & $\begin{array}{l}\text { with unfolding } \\
\text { events, } \\
\text { inflexible. }\end{array}$ & \\
\hline $\begin{array}{l}\text { "They are goal setting on their own now and } \\
\text { telling us what they want to do." }\end{array}$ & & & $\frac{\text { ELEMENTS }}{\text { Not Goal }}$ & \\
\hline & & & & \\
\hline
\end{tabular}

Figure 39: Theme 1 Goal Oriented, Willingness to Act and Perseverance the Hope Gap and Not Goal Oriented, Depleted Willpower, and Deficiency in Coping Skills 
On the left side are the manifestations of hope from the teachers' voices for the theme of Goal Oriented, Willingness to Act, and Perseverance. The textual evidence that displays hope in this theme is quoted on the left side. For example, Participant 1 stated, "I build a culture of having a goal." This demonstrates that the teacher understands the importance of students having goals and goals are connected to the element of Critical Hope through goal-directed thinking. On the right side are the manifestations of hopelessness, which emerged through the teachers' voices. Theme 1 is Not Goal Oriented, Depleted Willpower, and Deficiency in Coping skills. The textual evidence in Figure 40 shows educators who are hopeless in this theme and what they perceive as students who are hopeless in this theme as well. For example, Participant 10 stated, "I feel often that when kids feel they were abused, molested, or when they were given up for adoption, they can use that as an excuse, that they don't have to try anymore." This demonstrates that the students themselves have depleted willpower, as they often do not try inside the classroom. Therefore, there is a hope gap between those who set goals, are willing to act and persevere, and those who are not goal oriented, have depleted willpower, and a deficiency in coping skills. 


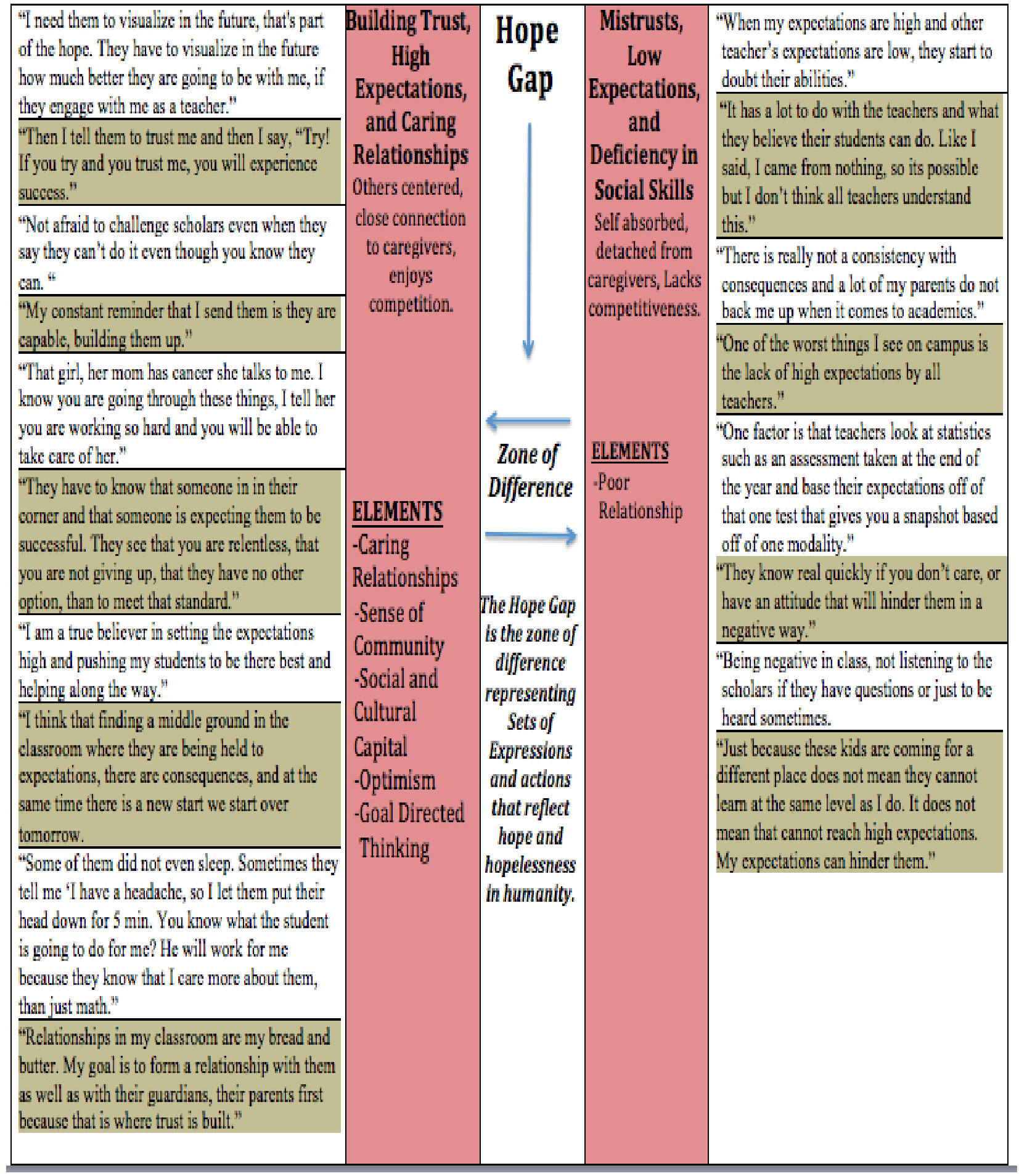

Figure 40: Theme 2 Building Trust, High Expectations, and Caring relationships, the Hope Gap, and Mistrust, Low Expectations, and Deficiency in Social Skills 
For Theme 2 Building Trust, High Expectations, and Caring relationships, it was noted by all the Participants the importance of establishing a caring relationship and trust that is built through that relationship as the most important factor in effective teaching. The high expectations were a result of the established caring relationships as, "caring teachers do not allow their students to not succeed" (Lalas et al., 2019, p. 48). For example, Participant 5 stated, "Relationships in my classroom are my bread and better. My goal is to form a relationship with them as well as with their guardians, their parents first because that is where trust is built." This teacher noted the importance of building relationships and trust with both the students and their guardians. On the other hand, the manifestations of hopelessness were also found in the theme of Mistrust, Low Expectations, and Deficiency in Social Skills. For example, Participant 10 stated, "There is not really a consistency with consequences and a lot of my parents do not back me up when it comes to academics." This demonstrates the inverse, as the teacher may have not effectively built caring relationships or established trust with the students or the parents. Thus, there is a hope gap between the two positions: hope and hopeless. 


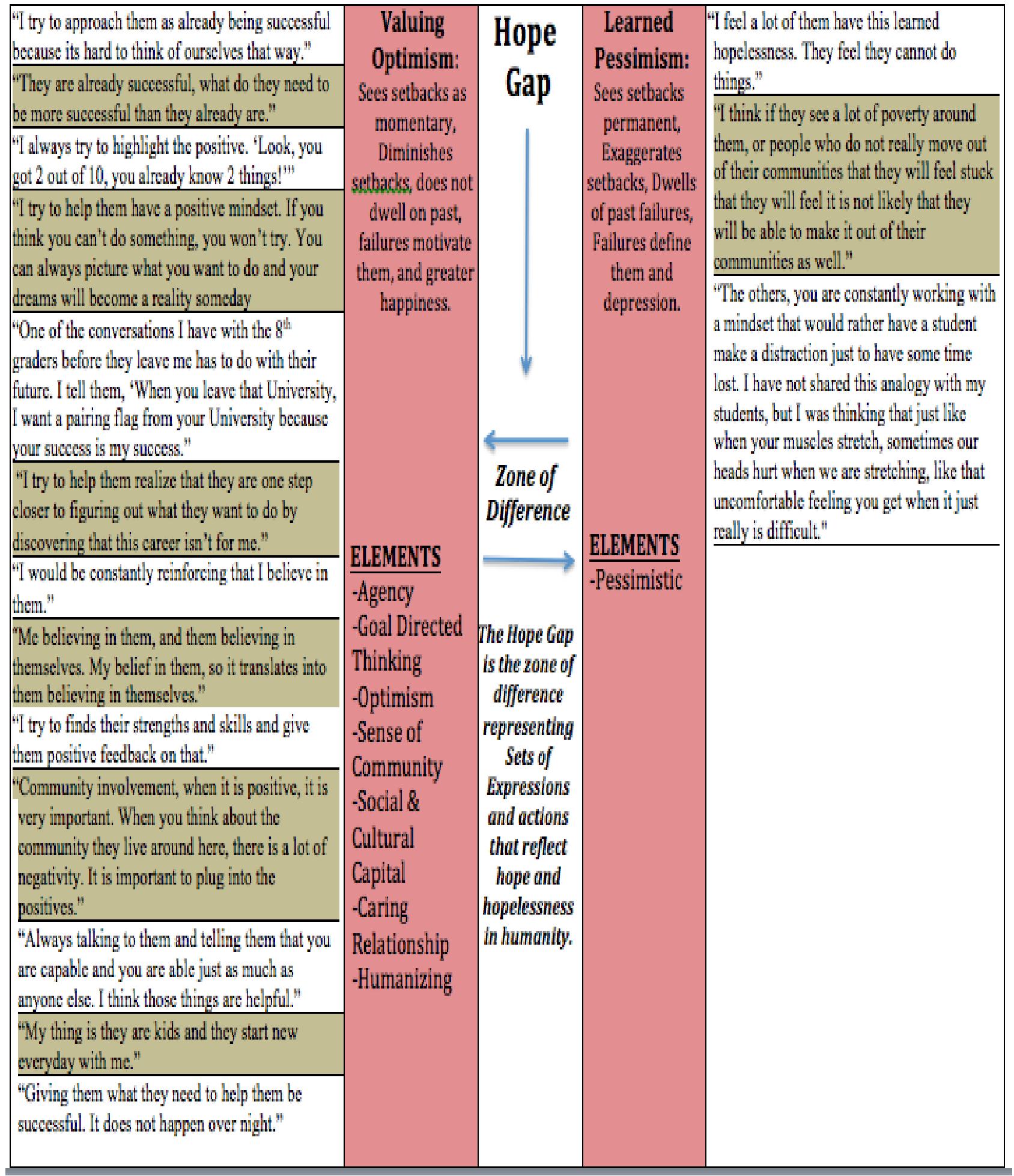

Figure 41: Theme 3: Valuing Optimism, the Hope Gap, and Learned Pessimism 
In Theme 3, the manifestations of Critical Hope emerged through teachers' voices in how they were Valuing Optimism. Teachers saw setbacks as momentary and used them to teach students how to persevere and be successful. They had an optimistic view of their students and believed deeply in their capabilities. For example, Participant 2 stated, "They are already successful, what do they need to be more successful." This optimism was very evident in the language and behavior of the hopeful teachers. On the other hand, manifestations of hopelessness were found through the theme of Learned Pessimism. For example, Participant 10 stated, "I feel a lot of them have this learned hopelessness. They feel they cannot do things." This demonstrates that the teacher views the students themselves as pessimistic and it prevents them from trying inside the classroom. Thus, there is a hope gap between the optimism and pessimism. 


\begin{tabular}{|c|c|c|c|c|}
\hline $\begin{array}{l}\text { "Not afrald to challenge scholars even waen they } \\
\text { say they can't do it even though you know they } \\
\text { can." } \\
\text { "I try to encourage them. I don't want them to look } \\
\text { at their present circumstances. I don't care where } \\
\text { you came from. I don't want you to say I did not } \\
\text { leam this because I came from King; I want them } \\
\text { to learn this well. They don't have to go to a } \\
\text { private school in a rich area; they can be } \\
\text { successful right where they arc..." } \\
\text { "I try to use their stories, to turn it around on how } \\
\text { they can be great, to empower them because } \\
\text { someone might use their story to demote them and } \\
\text { make them feel less than worthy to be on this } \\
\text { carth. I take that very same story and turn it } \\
\text { around, to help them feel like they have worth." } \\
\text { "I try to be a model, an example to them. } \\
\text { Throughout the year I tell them my own personal } \\
\text { stories that relate to them. How I got to this point } \\
\text { How I made my way. Things were not set up for } \\
\text { me to be successful and through my family and my } \\
\text { faith I made myself successful and they can do that } \\
\text { too." } \\
\text { "I try to give them stories about the things I have } \\
\text { done, about people I know, real life examples to } \\
\text { make things relevant and to let them know that } \\
\text { where you live doesn't define where you are } \\
\text { going." } \\
\text { "By making them see how I relate to them, by } \\
\text { making them see how I came from a similar } \\
\text { background and making them sec, for them to feel } \\
\text { suceessful to see how I grew up and my parents } \\
\text { grew up. If they were to see where I came from, } \\
\text { the neighborhood, the poverty, the discrimination } \\
\text { to where I am now, they can see that hope and } \\
\text { inspiration for them to see that you can be } \\
\text { successful in an upbringing that is similar to } \\
\text { mine." }\end{array}$ & $\begin{array}{c}\text { Confronting } \\
\text { Hopelessness } \\
\text { Through relevant } \\
\text { and authentic } \\
\text { personal } \\
\text { narratives, } \\
\text { relentless belief in } \\
\text { their capabilities } \\
\text { and } \\
\text { understanding. } \\
\\
\text { ELEMENTS } \\
\text {-Humanizing } \\
\text { Practices } \\
\text {-Social and } \\
\text { Historical } \\
\text { Consciousness } \\
\text {-Caring } \\
\text { Relationships }\end{array}$ & 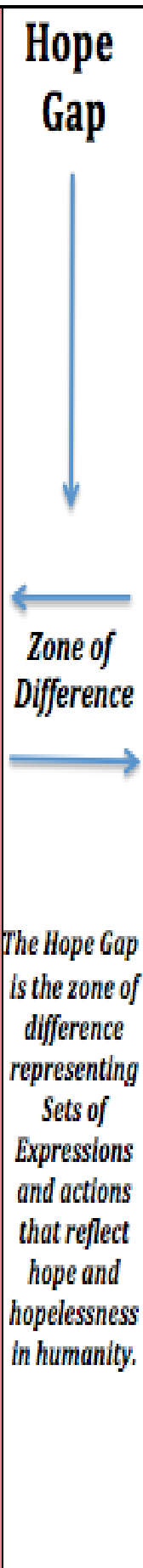 & & 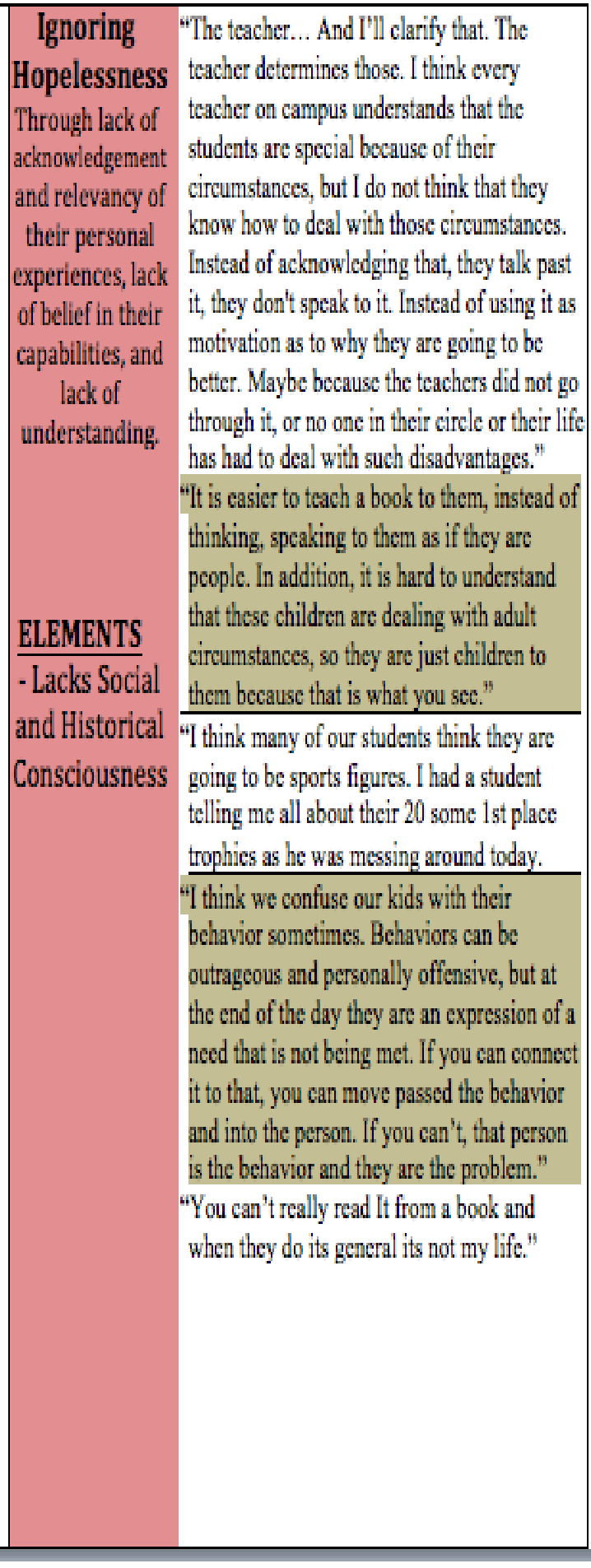 \\
\hline
\end{tabular}

Figure 42: Theme 4: Confronting Hopelessness, the Hope Gap, and Ignoring Hopelessness 
For Theme 4, manifestations of Critical Hope were found in the manner and ability of the teachers in Confronting Hopelessness through relevant and authentic personal narratives and in their relentless belief in their students' capabilities to be successful. For example, Participant 6 stated, "that even though students may resist, teachers need to not be afraid to challenge scholars even when they say they can’t do it, even though you know they can." This demonstrates the teacher being relentless and ensuring that students will reach their expectations. On the other hand, manifestations of hopelessness also emerged through teachers' voices in the theme of Ignoring Hopelessness through a lack of belief in their students' capabilities, lack of relevancy in content, and recognition of students' experiences inside the classroom. For example, Participant 5 stated, "I think every teacher on campus understands that the students are special because of their circumstances, but I do not think they know how to deal with those circumstances. Instead of acknowledging that, they talk past it, they don't speak to it." This demonstrates that educators who don't acknowledge what the students face on a daily basis are ignoring the hopelessness that they bring with them into the classroom. Thus, there is a hope gap between the two themes of confronting hopelessness and ignoring hopelessness. 


\begin{tabular}{|c|c|c|c|c|}
\hline $\begin{array}{l}\text { "I find myself being a counselor, a teacher, } \\
\text { disciplinarian, many other roles in middle school." }\end{array}$ & \multirow{9}{*}{$\begin{array}{c}\text { Personal } \\
\text { Commitment } \\
\text { to Ethical } \\
\text { Responsibility } \\
\text { and } \\
\text { Accountability } \\
\text { Positive adult } \\
\text { attitude, and holds } \\
\text { themselves } \\
\text { professionally and } \\
\text { personally } \\
\text { responsible to } \\
\text { whatever it takes } \\
\text { ensure student's } \\
\text { success. }\end{array}$} & \multirow{8}{*}{$\begin{array}{l}\text { Hope } \\
\text { Gap } \\
\\
\text { Difference }\end{array}$} & \multirow{8}{*}{\begin{tabular}{|c|} 
Professional \\
Deflection \\
and Lacks \\
Ethical \\
Accountability \\
Negative adult \\
attitude, does nit \\
hold \\
professionally or \\
personally \\
themselves \\
accountable to \\
ensure student \\
success.
\end{tabular}} & \multirow[t]{2}{*}{$\begin{array}{l}\text { "Being negative in class, not listening to the } \\
\text { scholars if they have questions or just to be } \\
\text { heard sometimes." }\end{array}$} \\
\hline "I think it has to do with more of you as a person. & & & & \\
\hline & & & & $\begin{array}{l}\text { "What drives us?' Is it a paycheck? Is it that I } \\
\text { want something to do?" }\end{array}$ \\
\hline students succeed then I know that I will have high & & & & Adults can feel that they have no bearing, \\
\hline & & & & \\
\hline $\begin{array}{l}\text { Demonstration first! I have to have faith, so I have } \\
\text { to have hope and confidence in them, in their }\end{array}$ & & & & $\begin{array}{l}\text { they take responsibility that they are doing } \\
\text { well." }\end{array}$ \\
\hline $\begin{array}{l}\text { abilities first because a lot of them do not believe } \\
\text { in themselves. I have to have almost an } \\
\text { exaggerated amount of hope, so they buy in." }\end{array}$ & & & & $\begin{array}{l}\text { If I am focused and concentrated on teaching } \\
\text { but not focused on if they are learning then } \\
\text { that can hinder their success. Also if I have }\end{array}$ \\
\hline $\begin{array}{l}\text { In general, show you want to be more than just a } \\
\text { teacher, be active in the community, a part of their } \\
\text { culture in the community, in the school, and be } \\
\text { hopeful. That is when you have complete buy in. } \\
\text { lt's hard but once you get the parents trust that } \\
\text { opens the doors for every thing else." }\end{array}$ & & & & $\begin{array}{l}\text { low expectations. Just because these kids are } \\
\text { coming for a different place does not mean } \\
\text { they cannot learn at the same level as I do. It } \\
\text { does not mean that cannot reach high } \\
\text { expectations. My expectations can hinder } \\
\text { them." }\end{array}$ \\
\hline $\begin{array}{l}\text { Even when they do something small, I have to } \\
\text { show them and make it a big deal. Wow!!this is } \\
\text { amazing!! Wow!! Then after a few months they } \\
\text { start using my language. I have to make sure that } \\
\text { my jar of hope is full and running over because }\end{array}$ & & & ELEMENTS & $\begin{array}{l}\text { "I have to think about that in terms of History. } \\
\text { I'm not sure directly, you know its not a } \\
\text { carcer class. (I'm not liking your survey its } \\
\text { making me uncomfortable. I think I could do } \\
\text { a better job...." }\end{array}$ \\
\hline $\begin{array}{l}\text { they might not see hope in their homes, or on the } \\
\text { strect. I have to be that demonstration, that ray of } \\
\text { light, when everyone who is around them is telling }\end{array}$ & $\begin{array}{l}\text {-Ethics } \\
\text {-Sense } 0\end{array}$ & $\begin{array}{l}\text { difference } \\
\text { representing } \\
\text { Sets of }\end{array}$ & -Unethical & $\begin{array}{l}\text { "Being negative in class, not listening to the } \\
\text { scholars if they have questions or just to be } \\
\text { heard sometimes." }\end{array}$ \\
\hline them something different." & Community & & & "I think a lot of times people don't want to \\
\hline $\begin{array}{l}\text { "If they are hungry, I can choose to provide } \\
\text { something for them. When I bring all these } \\
\text { oranges I don't bring them just for myself. I know } \\
\text { I have students who haven't caten The granola } \\
\text { bars; the students have been cating these bars. } \\
\text { Because they don't have breakfast. Making sure } \\
\text { they are eating well." }\end{array}$ & & $\begin{array}{l}\text { and actions } \\
\text { that reflect } \\
\text { hope and } \\
\text { hopelessness } \\
\text { in humanity. }\end{array}$ & & $\begin{array}{l}\text { poke the bear with those who have behavior } \\
\text { issues, so they just let them fall to the } \\
\text { wayside. There is really not a consistency } \\
\text { with consequences." }\end{array}$ \\
\hline
\end{tabular}

Figure 43: Theme 5 Personal Commitment to Ethical Responsibility and Accountability, the Hope Gap, and Professional Deflection and Lacks Ethical Accountability

In Theme 5, manifestations of Critical Hope emerged through Personal Commitment to Ethical Responsibility and Accountability. This is where the adults hold themselves personally 
responsible and accountable to students' success. They do whatever it takes to ensure their students meet their high expectations. For example, Participant 8 stated,

I think it has to do with more of who you are as a person. What is your goal? What is your motive for teaching? What is your why? If my why is to see my students succeed, then I know that I will have high expectations. If they are not learning, the responsibility involves me.

This demonstrates the personal and ethical responsibility educators have to ensuring student success. On the other hand, there were also manifestations of hopelessness in the inverse theme of Professional Deflection and Lacks Ethical Accountability where teachers did not take responsibility but rather blame shifted that responsibility onto others like students, parents, past educators, etc. One teacher actually noted being very uncomfortable with this question. It was a simple interview question that asked: What learning opportunities do you provide your students that would strengthen their desire to be successful in the fields they are interested in? Participant 9 responded with personal deflection-and stated, "I have to think of that in terms of History. I'm not sure directly, you know it's not a career class." Then the Participant went onto to say, "I'm not liking your survey, and it's making me uncomfortable. I think I could do a better job." This demonstrates that the first response was personal deflection and somewhere in between the Participant felt remorse for not making connections to fields students are interested in. Thus, there is a gap between the manifestations of Critical Hope in Theme 5: Personal Commitment to Ethical Responsibility and Accountability and the manifestations of hopelessness in Professional Deflection and Lacks Ethical Accountability. 


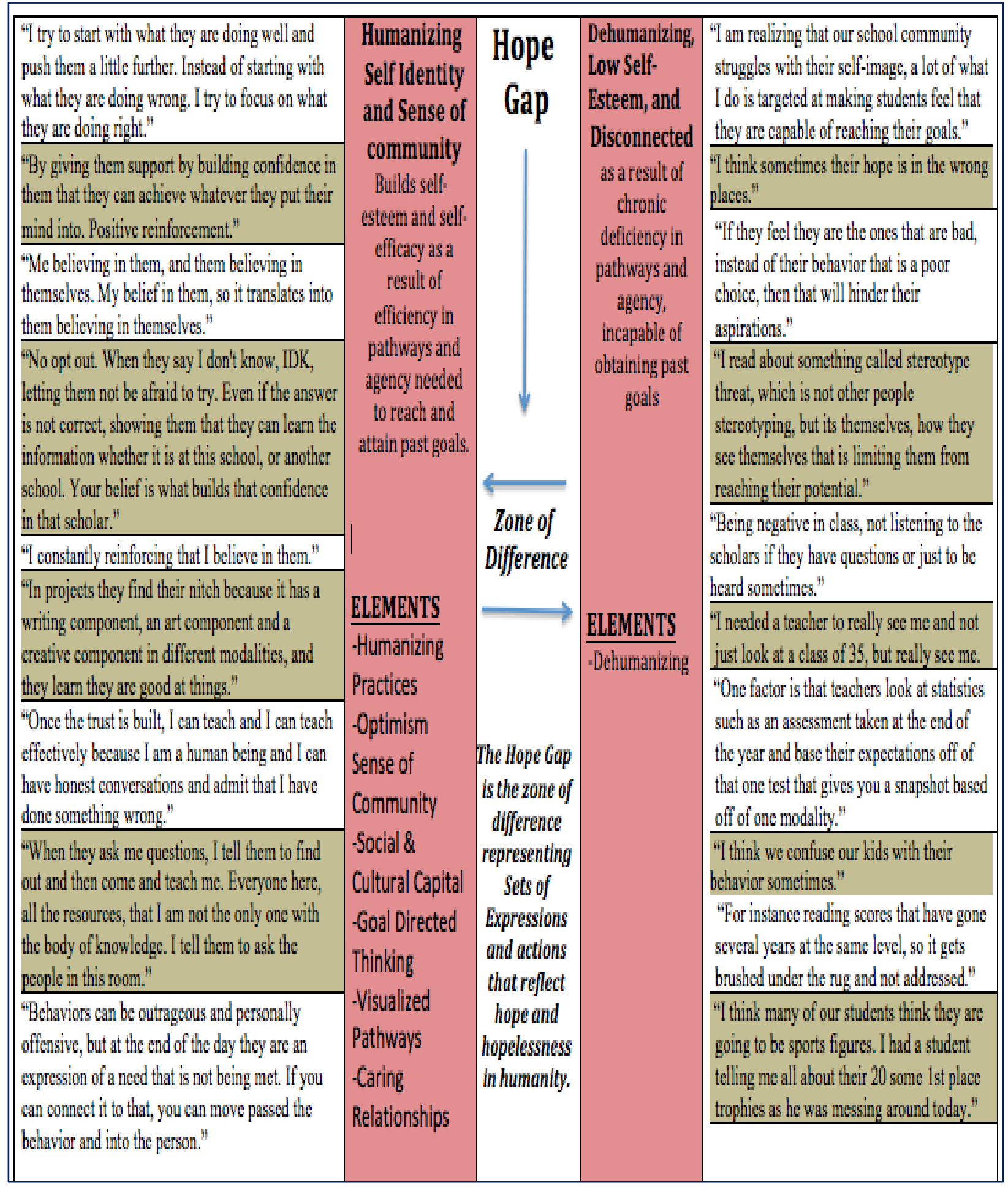

Figure 44: Theme 6 Humanizing Self Identity and Sense of Community, the Hope Gap, and Dehumanizing, Low-Self Esteem, and Disconnected 
For Theme 6: Manifestations of Critical Hope is found in Humanizing Self Identity and Sense of Community where the educator provided opportunities for students to have dialogue, reflection, and praxis in the classroom, which in turn created a sense of belonging in the classroom between the students and the teacher and increased the students' self-identity. Teachers believed in their students' abilities and acknowledged that students have knowledge that should be expressed and valued inside the classroom. For example, Participant 8 stated, "When they ask me questions, I tell them to find out and then come teach me. Everyone in here, all the resources, that I am not the only one with the body of knowledge. I tell them to ask the people in this room." This demonstrates that the teacher wants to encourage the students to see themselves as knowledgeable and encourages discourse between the students, which is humanizing for students of poverty who have been demoralized. On the other hand, manifestations of hopelessness were found in the theme of Dehumanizing, Low-Self Esteem, and Disconnected as the Participants noted how educators can dehumanize students by not seeing them or not hearing their voices, and by labeling students as Participant 2 stated, "I think we confuse our students with their behavior sometimes." We label them as behavioral students instead of as a "student who is making a poor choice." Thus, there is a hope gap in Theme 6 between the manifestations of Critical Hope in Humanizing Self Identity and Sense of Community, and the manifestations of hopelessness in Dehumanizing, Low-Self Esteem, and Disconnected. 


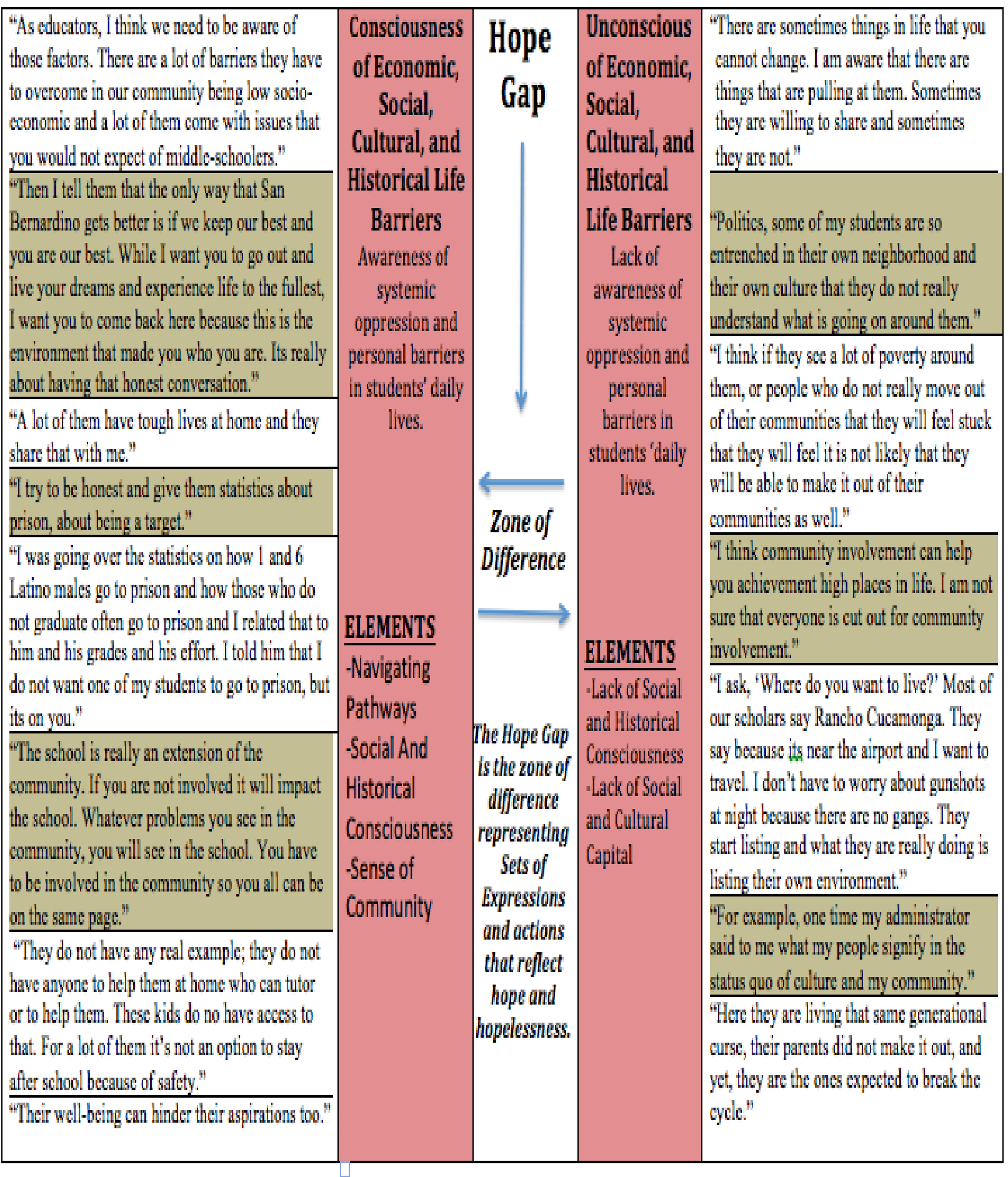

Figure 45: Theme 7 Consciousness of Economic, Social, Cultural, and Historical Life Barriers, the Hope Gap, and Unconscious of Economic, Social, Cultural, and Historical Life Barriers 
EXAMINING TEACHERS' PARADIGM AND PEDAGOGY IN MANIFESTING CRITICAL HOPE IN LOW-INCOME MIDDLE SCHOOL

In Theme 7, manifestations of Critical Hope emerged in the theme of Consciousness of Economic, Social, Cultural, and Historical Life Barriers as Participants noted and were very aware at times of the out-of-school factors (Berliner, 2005) that may affect a student's hope in their quest to be successful. For example, Participant 1 stated,

As educators, I think we need to be aware of those factors. There are a lot of barriers they have to overcome in our community being low socioeconomic and a lot of them come with issues that you would not expect of a middle schooler.

This demonstrates the consciousness this educator has of these life barriers and the importance of the understanding it brings into the classroom. Inversely, manifestations of hopelessness were also found as through the teachers' voices emerged that educators, students, and parents can also be Unconscious of Economic, Social, Cultural, and Historical Life Barriers. For example, Participant 5 stated, "Here they are living that same generational curse. Their parents did not make it out, and yet they are ones expected to break the cycle." This quote is powerful because if we are unconscious of economic, social, cultural, and historical life barriers, and there is this expectation that students are super agents (Berliner, 2005), then the systemic oppression will continue to create generational poverty as we cannot overestimate the power of the person and underestimate the power of the situation. This will only lead to hopelessness and despair (Freire, 1994). Thus, there is a hope gap between those who are consciousness of economic, social, cultural, and historical life barriers and those who are unconscious of economic, social, cultural, and historical life barriers. 


\begin{tabular}{|c|c|c|c|c|}
\hline 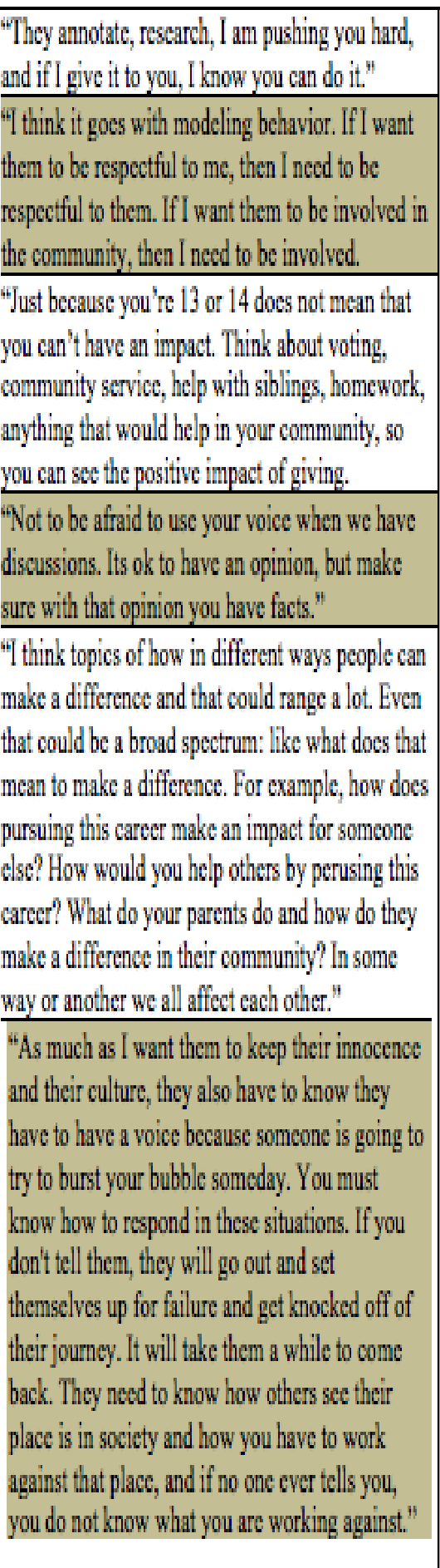 & $\begin{array}{c}\text { Critical } \\
\text { Reflection, } \\
\text { Advocacy, } \\
\text { Citizenry, and } \\
\text { Empowerment } \\
\text { Talkes bold risks, } \\
\text { accepts challenges, } \\
\text { enjoys trying new } \\
\text { thinge and is } \\
\text { attracted to the } \\
\text { process of testing } \\
\text { and strutching } \\
\text { their skills. }\end{array}$ & $\begin{array}{l}\text { The Hope Gap } \\
\text { is the zone of } \\
\text { difference } \\
\text { representing } \\
\text { Sets of } \\
\text { Expressions } \\
\text { and actions } \\
\text { that reflect } \\
\text { hope and } \\
\text { hopelessness } \\
\text { in huminanity. }\end{array}$ & 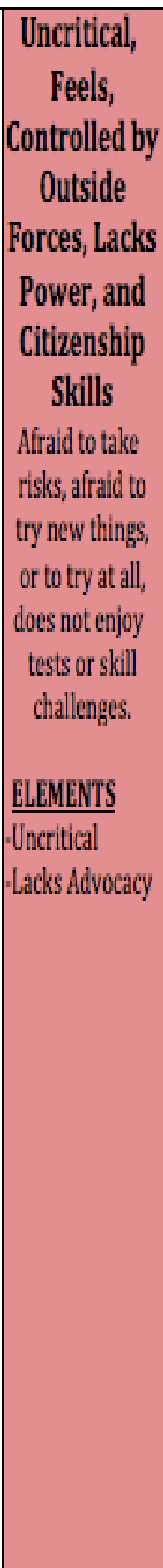 & 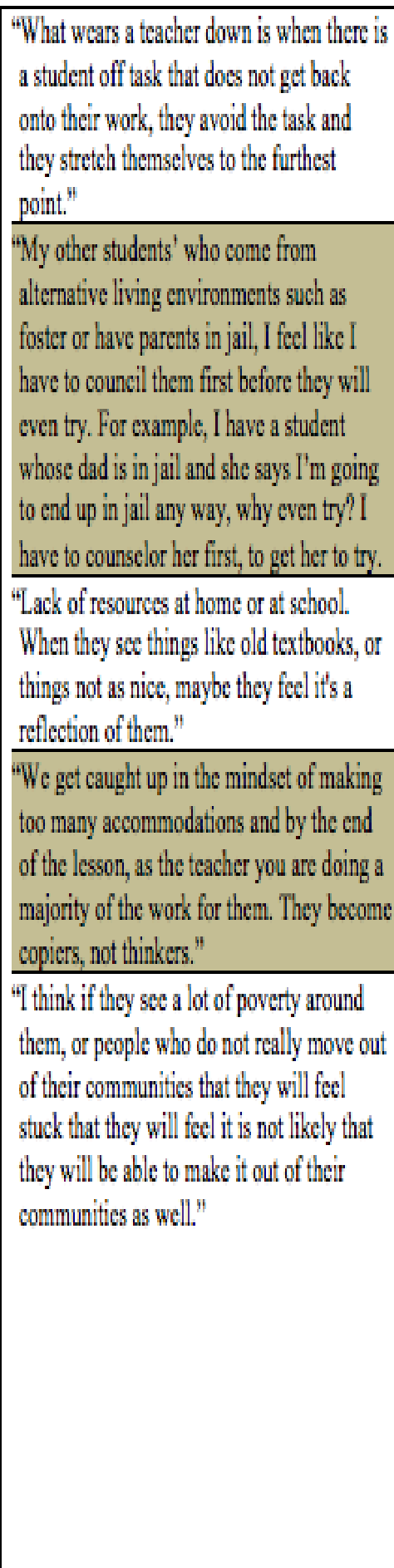 \\
\hline
\end{tabular}

Figure 46: Theme 8 Critical Reflection, Advocacy, Citizenry, and Empowerment, the Hope Gap, and Uncritical, Feels Controlled by Outside Forces, Lacks Power, and Citizenship Skills 
For Theme 8, manifestations of Critical Hope were found in Critical Reflection, Advocacy, Citizenry, and Empowerment as educators noted the importance of empowering students to take bold risks, to use their minds to think critically, and to advocate for change. For example, Participant 6 stated, "I teach my students to not be afraid to use your voice when we have discussions. It's ok to have an opinion, but make sure with that opinion you have facts." This demonstrates the teacher's emphasis on student voice and critical thinking inside the classroom. On the other hand, manifestations of hopelessness were also found in the theme of Uncritical, Feels Controlled by Outside Forces, Lacks Power, and Citizenship Skills. This occurs when students feel controlled by outside forces, they are not expected to think critically inside the classroom and they lack power and citizenship skill. For example, Participant 1 stated, "We get caught up in the mindset of making too many accommodations and by the end of the lesson, as the teacher you are doing a majority of the work for them. They become copiers not thinkers." This demonstrates that a lack of belief in students' abilities by the educator produces tasks that are uncritical and lack power. Students sit idly by and become copiers and they do not learn how to think for themselves. Thus, there is a hope gap in Theme 8 of Critical Reflection, Advocacy, Citizenry, and Empowerment, and Uncritical, Feels Controlled by Outside Forces, Lacks Power, and Citizenship Skills. 


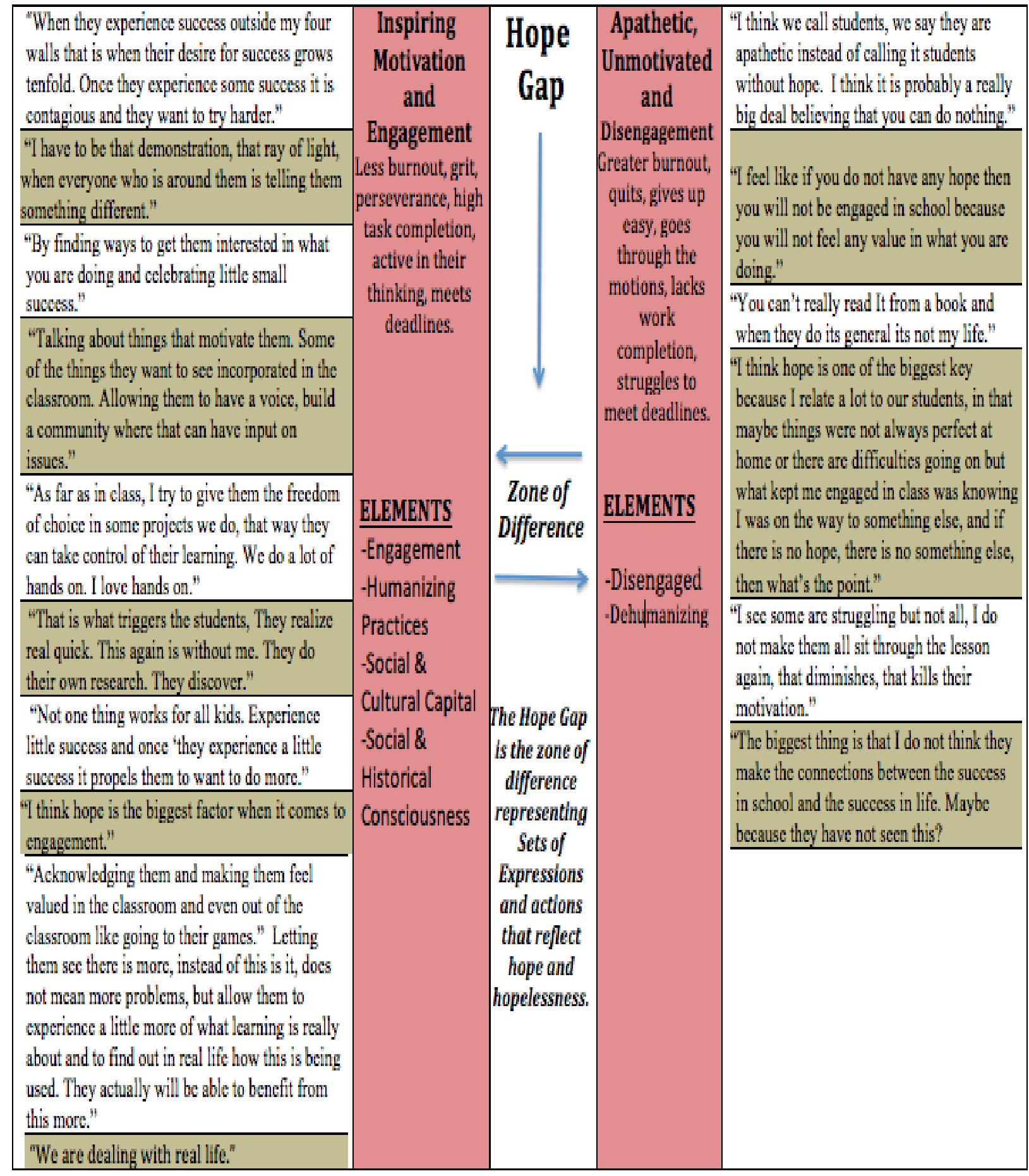

Figure 47: Theme 9 Inspiring Motivation and Engagement, the Hope Gap, and Apathetic, Unmotivated, and Disengagement 
EXAMINING TEACHERS' PARADIGM AND PEDAGOGY IN MANIFESTING CRITICAL HOPE IN LOW-INCOME MIDDLE SCHOOL

In Theme 9, manifestations of Critical Hope were found in Inspiring Motivation and Engagement, as the educators were very inspirational in their language and behavior of hope inside the classroom and in the interviews. You could feel their passion and they connected with the four factors of motivation that research has stated as sense of belonging, autonomy, competence, and meaningfulness, which leads to student engagement (Lalas et al., 2019). For example, Participant 8 stated, "Talking about things that motivate them. Some of the things that they want to see incorporated into the classroom. Allowing them to have a voice. Building a community where they can have input on things." This demonstrates the connection between motivation and student engagement through autonomy, competency, meaningfulness, and sense of belonging. The Participants also noted that hope is a big factor in student engagement. For example, Participant 2 states, "I think hope is the biggest factor when it comes to engagement." Contrarily, manifestations of hopelessness also emerged through the voices of teachers in the theme of Apathetic, Unmotivated, and Disengagement where content was not meaningful or relevant to students, where they had no choice or belief in their capabilities, and as a result, students were viewed as unmotivated or apathetic. Teachers also noted that without hope students will not be engaged. Participant 2 stated, "I think that if you do not have any hope you will not be engaged in school because you will not feel any value in what you are doing." The value in what you are doing is connected to motivation as meaningfulness. If students are hopeless, it may be because the tasks are meaningless to them and what they are experiencing in their everyday lives. Thus, there is a disconnect and a hope gap between the two themes of Inspiring Motivation and Engagement, and Apathetic, Unmotivated, and Disengagement. 


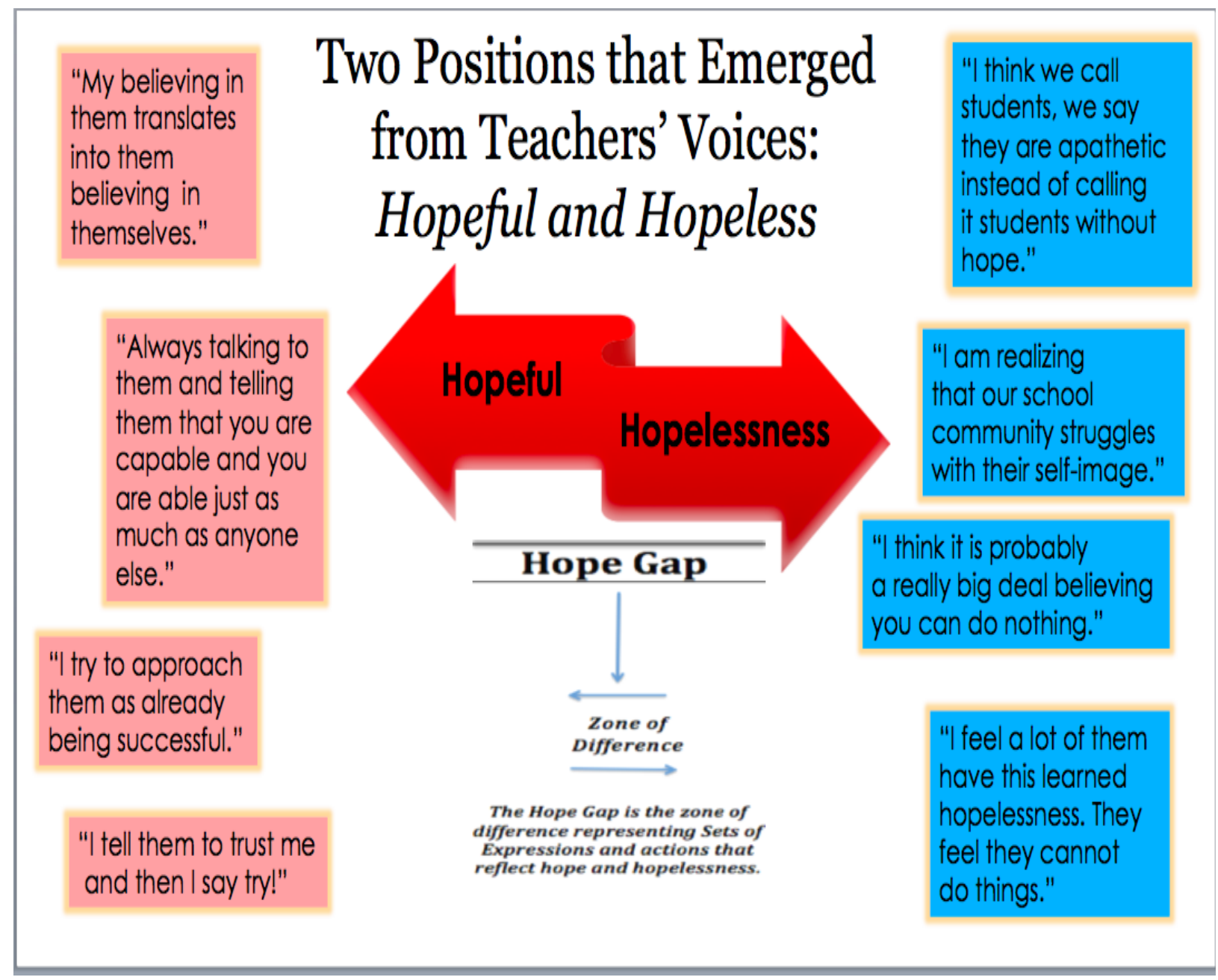

Figure 48: Two Positions that Emerged from Teachers' Voices: Hopeful and the Hopeless as a Visual Display of the Hope Gap

In Figure 48, the voices of the teachers of a low-income middle school demonstrate the hope gap. As Participant 6 stated, "My believing in them translates into them believing in themselves." This demonstrates the teachers' awareness that the students have a low belief in their abilities and when the teacher believes in them, the student's hope is raised and they begin to believe in their own abilities. This is contrasted with Participant 9's statement on the top right that states, "I think we call students; we say they are apathetic instead of calling it student's without hope." This teacher is also aware of the lack of hope the students have, but instead of 
confronting the hopelessness with belief in their abilities, the teacher continues the hopelessness by labeling students as apathetic or without hope. This is an example of unintentional hopelessness of unstable and oppressive environments through labeling. Thus, the hope gap is real and apparent and may reside in both the educators and in the students themselves.

\section{Participants}

The Participants are all teachers at a low-income middle school in an urban area with high crime. The middle school's data mirrors the data for the city it is located in.

\section{Demographics}

\section{Middle School}

- Total enrollment: 704

- Free and reduced lunch: $100 \%$

- Homeless \& Foster Youth $15 \%$

- English Learner $27 \%$

- Hispanic $81.4 \%$

- African American $11.5 \%$

- White $2.9 \%$

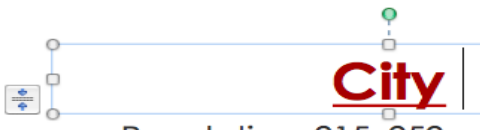

- Population: 215, 252

- Ranked $9^{\text {th }}$ Worst City to live in Ca.

- Medium Home Value: \$202,100

- Unemployment: $12.8 \%$

- 1 in 10 residents are without a job

- Crime Rate: $88 \%$ higher than the national average.

- 1 in 20 chance of becoming a victim of crime

Figure 49: Site Demographics

The teachers were recruited to participate in the study because they are all middle school teachers in the same low-income school. The sample was purposive sampling due to the important qualities of the targeted population (Etikan et al., 2016). All potential teacher Participants were on the same campus that we will call Middle School (MS) as a pseudonym. Depending upon interest, some or all of the Participants were selected to provide an unbiased 
sample of teachers. The intent was not to make any generalizations of the findings, but rather to provide a variety of teachers' voice in all content areas and grade levels to support the study's purpose and rationale (Patton, 2002). To determine teacher-Participants' current level of hopefulness, the Lopez Hope Scale (Lopez, 2013) was used as a qualitative descriptor of Participants. Teachers who were recruited are referred to by alphanumeric system, which identifies the Participants by a combination of alphabetic and numeric characters to keep their identities private. This process was beneficial to connect information to the teachers while keeping their identities private and protected.

\begin{tabular}{|l|l|l|l|}
\hline Participants & Subject/Grade Level & Years of Teaching Experience & Hope Score \\
\hline Participant 1 & AVID 7th/8th & 6yrs: 5yrs King/1yr Colton & $20 / 2 / 14$ \\
Participant 2 & Science 8th & 2yrs:2yrs King & $23 / 9 / 15$ \\
Participant 3 & Math 8th & 1st year-1yr King & $24 / 4 / 13$ \\
Participant 4 & History 8th & 5yrs: 3yrs King/2yrs in China & $24 / 9 / 14$ \\
Participant 5 & ELD 7th/8th & 6yrs: 2 yrs King/ 3yrs Juvenile Hall/1yr continuation SCUSD & $22 / 5 / 15$ \\
Participant 6 & History 8th & 6yrs: 4yrs King/2yrs private St. Josephs Fontana & $22 / 8 / 15$ \\
Participant 7 & Science 7th & 1st yr: 1yr King/2yrs Els tutor at Arroryo & $20 / 4 / 11$ \\
Participant 8 & Math 8th & 19yrs:18yrs King/ 1yr Shandon Hills & $23 / 2 / 15$ \\
Participant 9 & History 7th & 30yrs: 4yrs King/26yrs Del Velejo & $21 / 5 / 12$ \\
Participant 10 & SPED ELA/History 7th/8th & 1.5yrs King started Feb 2018 & $21 / 4 / 12$ \\
\hline
\end{tabular}

Figure 50: Participants

Generally, all teachers scored fairly high on the Lopez Hope Scale (Lopez, 2013), which is not surprising considering the area where the teachers have chosen to work. I found the hope scale to not be useful in this study as it did not define the Participants or set them apart in any 
way. In Figure 50, you can see that each Participant scored 20 or higher on the first section which calculated their hope score. According to the Lopez (2013) scale, if you scored 21 or above, you are a high-hope person whose thinking about the future is an asset. The second set is 'This is your readiness to Hope Score,' ranging from 2 to 10 . The higher you score, the more you believe that your future is dominated by your past and present circumstances, and the less room you have for hope. The third section is the Hope Contagion score, ranging from 3 to 15 . If they scored above 12 , they are a model for others and consciously boost the hope of those around you. As you can see, the range of scores in all three areas is relatively close and hopeful from all the Participants.

This site is located in the highest crime area in southern California. According to a recent article $^{1}$ this urban city was found to be in the top 10 of the worst and most dangerous places to live in California because of the high crime rate. During this school year, when the study was conducted from August 1, 2018 - June 7, 2019, there were over 3,500 crimes reported in a twomile radius around the school. ${ }^{1}$ As Freire (1994) stated, "It is imperative that we maintain hope even when the harshness of reality may suggest the opposite (p. 4)". The educators of this school have been trained to be aware of the crime and the impact it has on students. They have all gone through trauma-sensitive training. Thus, I am not surprised that the educators at this school site have personal high levels of hope according to the Lopez Hope Scale (Lopez, 2013).

Participant 1: Has been teaching at MS for six years. This teacher is an AVID teacher and teaches both 7 th and 8th graders. This teacher is a part of the site leadership on campus and knows the student population well. Participant 1 defined hope as, "Building a personality with

\footnotetext{
${ }^{1}$ Further identification withheld for confidentiality
} 
perseverance, integrity, to set goals, and see those goals through. Believing as an educator they are capable. They need to believe and I need to believe."

Participant 2: Has been teaching at MS for two years. This teacher teaches 8 th grade Science and is a part of the collegiality committee. Participant 2 defined hope as "The courage to dream and to pursue those dreams."

Participant 3: Has only been teaching for one year. This teacher teaches 8 th grade math and is from the same area. This teacher attended MS for their middle school experience. Participant 3 defined hope as, "Having a positive mindset toward a situation, something you want, or need."

Participant 4: Has been teaching for five years; three of them at MS and two were in China. This teacher teaches 8th grade History and leads the African American Bee on campus. Participant 4 defined hope as, "Being positive and having a positive spirit. Always having something to look forward to. Always feeling like you can make a difference."

Participant 5: Has been teaching for six years and has both continuation and juvenile hall experience. This teacher teaches 7th and 8th grade English Language Development. Participant 5 defined hope as, "Believing in the unseen. My hope is my faith. I really don't say hope, I say faith."

Participant 6: Has been teaching for six years. This teacher teaches 8th grade History and is also the technical support on campus. Participant 6 defined hope as, "To believe in and never giving up."

Participant 7: Has been teaching for one year. This teacher teaches 7th grade Science and is from the area. Participant 7 defined hope as 
Seeing in the future that there is always something, that chance, that possibility for change, so that nothing is set in stone. Anything in the future is pliable; you can change it if you want to. Having the desire for something.

Participant 8: Has been teaching for 19 years, all but one year have been at MS. This teacher teaches 8th grade Math, is the Math Department Chair, and a Math demonstration teacher for the district. Participant 8 defined hope as

Hope is when you see something that is not right in front of you. You don't have it right now, but you have the assurance and the confidence that you are going to get this.

Knowing that there is something for me tomorrow.

Participant 9: Has been teaching for 30 years, only four have been at MS. This teacher teaches 7 th grade History and is the History Department Chair. Participant 9 defined hope as, "There are good things ahead in your future that will give you joy, that your life can be meaningful, that you can be a benefit and blessing to others, and that you have some control over the outcomes."

Participant 10: Has been teaching for one and a half years. This teacher teaches Special Education in the subjects of English and History. Participant defined hope as, "Believing that you are capable of achieving your goals no matter what is happening around you and knowing that there is more."

\section{Shift in Conceptual Framework}

After examining the manifestations of Critical Hope in teachers' paradigm and pedagogy, I discovered that the paradigm of hope (asset based) drives the language and behavior of hope, the instructional delivery of hope, and the lesson planning of hope, and those with a paradigm of hopelessness (deficit based) drives the language and behavior of hopelessness, instructional 
delivery of hopelessness, and lesson planning of hopelessness. This discovery enforces the understanding that educators are driven to teach the way they think and believe, as those who have a hopeful paradigm will result in hopeful teaching practices and those who have a hopeless paradigm will result in hopeless teaching practices. Research has shown that human behaviors are driven by their ideology and influenced by their perceptions of the world (Johnson, 2007). Thus, paradigm and pedagogy are inseparable, which causes a shift in my conceptual framework to reflect this discovery. See Figure 51 for new conceptual framework.

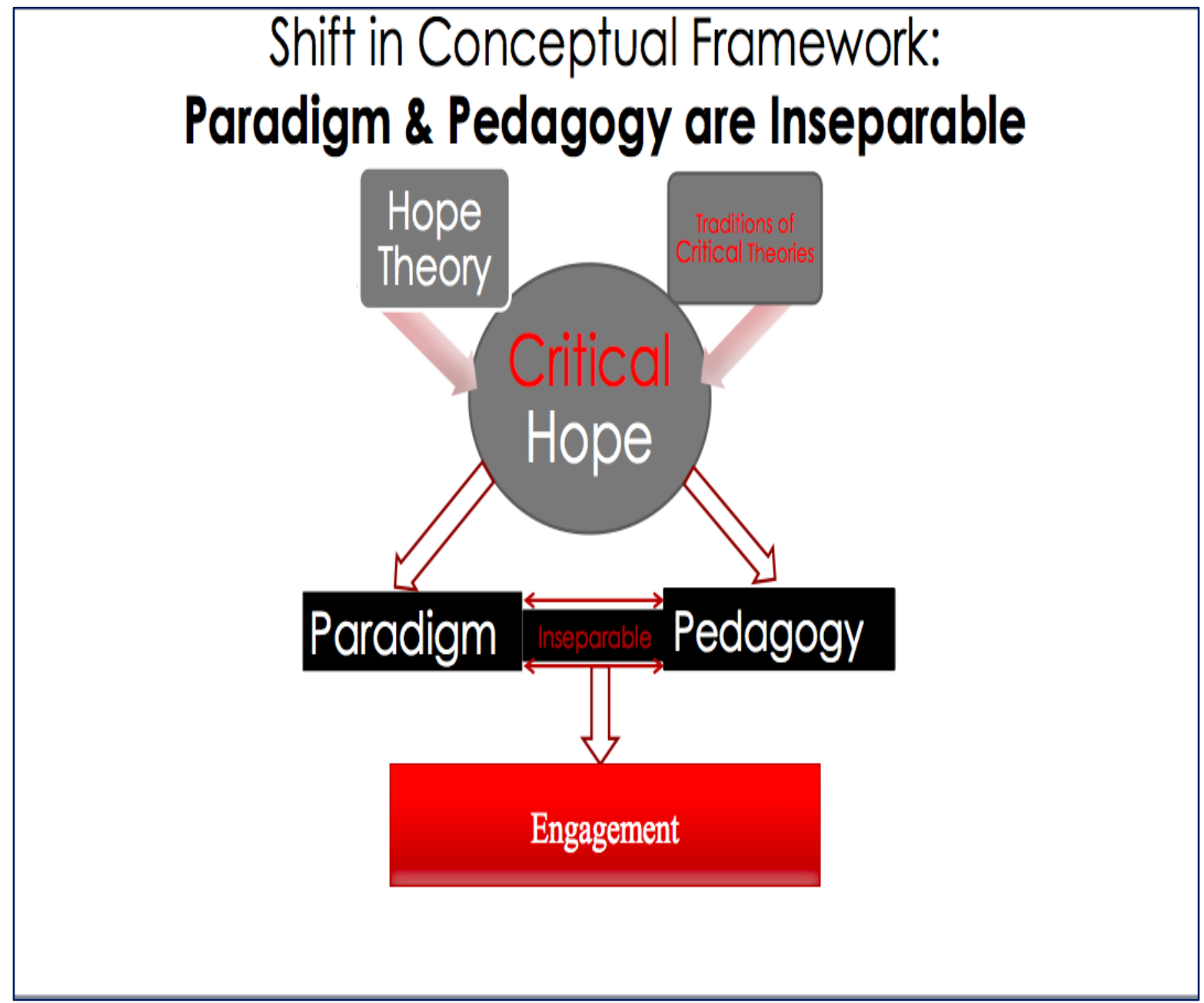

Figure 51: New Conceptual Framework 
EXAMINING TEACHERS' PARADIGM AND PEDAGOGY IN MANIFESTING CRITICAL HOPE IN LOW-INCOME MIDDLE SCHOOL

\section{Chapter Five. Conclusion \\ Summary of Study}

Chapter one of this dissertation study included an explanation of the problem of the achievement gap that exists between low-income students and affluent students and English Learners and English-Only students. Looking at national, state, and local data, there is an achievement gap between low-income students and affluent students, there are also inequities found in funding, and teacher quality between low-income students and affluent students all which constitutes a social-justice issue. Using Critical Hope as the conceptual framework, the researcher sought to answer the study's research questions through a qualitative narrative inquiry examining the manifestations of Critical Hope in teachers' paradigm and pedagogy in lowincome middle school. Based on this conceptual framework, the researcher answered the following questions: To what extent do the voices of teachers of low-income students reflect Critical Hope in their paradigm and pedagogy? What themes related to teachers' language and behavior of hope, teachers' instructional delivery of hope, teachers' instructional planning of hope emerge when observing and listening to teachers? What recommendations for educators can be made to reflect the manifestations of "Critical Hope" in teachers' paradigm and pedagogy?

Chapter two included the foundational theories and empirical studies behind poverty, paradigm, pedagogy, critical traditions of educational practices, hope, motivation, and engagement. The gap in literature is found on the manifestations of Critical Hope in teachers' paradigm and pedagogy in low-income middle school. To address this gap, the narrative inquiry qualitative method was chosen. 
EXAMINING TEACHERS' PARADIGM AND PEDAGOGY IN MANIFESTING CRITICAL HOPE IN LOW-INCOME MIDDLE SCHOOL

In chapter three the study's methodology was the focus. Using Seidman's (2006) threetiered approach to interviewing and purposive sampling, 10 teacher Participants were interviewed and observed for this study. Their years of teaching experience ranged from one year to 30 years and they represented each grade level and all content disciplines found on campus. All 10 Participants were selected from the same middle school site and all interviews were conducted at this site. Over the course of two weeks, each Participant was interviewed and observed using the interview protocol and developed interview prompts and observation matrix developed by the researcher. The structure of the interviews was semi structured for a more conversational interview with open-ended questions and follow-up questions to provide a deep understanding of the critical issue of hope through teachers' voices.

Chapter four included a discussion on how the researcher transcribed all 10 Participants interviews and hand coded each document several times using NVivo as a tool. The researcher used open coding to create axial codes, and axial codes to create analytical themes. Nine themes were found in the manifestations of Critical Hope in teachers' paradigm and pedagogy. The data was uploaded into NVivo software program as a tool for a word query on the definition of hope through the teachers' voices. This led to the discovery that hope is very personal to all the Participants. Thus, the definition of Critical Hope went through the process of design, designing and redesigned to include the notion that Critical Hope is a personal belief, and has been informed by an extensive literature review. In addition, incidental findings of hopelessness were found discovering two positions: the hopeful and the hopeless. Consequently, nine themes were discovered for the inverse of hopelessness. A second incidental finding was found in the area of unintentional hopelessness that is created by educators who are unaware of how common teaching practices and beliefs may lead to acts of symbolic violence, misrecognition, unstable 
EXAMINING TEACHERS' PARADIGM AND PEDAGOGY IN MANIFESTING CRITICAL HOPE IN LOW-INCOME MIDDLE SCHOOL

and oppressive environments, self-deception, self-depreciation, and narrative sickness. These incidental finding led to the discovery that the hope gap exists which I defined as the zone of difference representing sets of expressions and actions that reflect hope and hopelessness in humanity.

Once the data was collected, the researcher provided textual evidence for each theme and category along with the connection to Critical Hope elements. To explain the development of the manifestations of Critical Hope, the hope gap and hopelessness, the researcher wrote nine explanations, one for each theme highlighting the hope gap in teachers' paradigm and pedagogy. This led to a shift in conceptual framework to include the findings, which support that teachers' paradigm and pedagogy are inseparable.

Chapter Five of this study includes a summary of the study, a brief review of the research questions, methodology, data analysis, and the findings. Also included in this chapter are the recommendations for professional development pertaining to Critical Hope and future research.

\section{Research Questions Discussion}

Based on this conceptual framework, this paper sought to answer the following questions: To what extent do the voices of teachers of low-income students reflect Critical Hope in their paradigm and pedagogy? What themes related to teachers' language and behavior of hope, teachers' instructional delivery of hope, teachers' instructional planning of hope emerge when observing and listening to teachers? What recommendations for educators can be made to reflect the manifestations of "Critical Hope" in teachers' paradigm and pedagogy?

In this study, I learned that hope is very personal to the individual and it is connected to a belief in something that is valuable to the person. In answering question one, the extent of the voices of teacher reflects Critical Hope elements, were directly related to their paradigm of hope 
or paradigm of hopelessness. What makes Critical Hope really critical is the awareness of the social, cultural, economic, political, and historical factors that influence one's level of hope and the process of reflection and acting in ways that bring transformation of the world. In answering research question two, the findings from the 10 Participant interviews pointed toward the elements of Critical Hope such as Goals, Pathways, Agency, Optimism, Ethics, Social and Historical Consciousness, Caring Relationships, Sense of Community, Social and Cultural Capital, Humanizing Practices (Criticality, Dialogue, Praxis), Citizenry, and Democratic Advocacy, which are embedded in the following nine themes of Critical Hope that emerged:

- $\quad$ Building Trust, High Expectations and Caring Relationships

- Goal Oriented, Willingness to Act, and Persevere

- Critical Reflection, Advocacy, Citizenry, and Empowerment

- $\quad$ Valuing Optimism

- Confronting Hopelessness

- Personal Commitment to Ethical Responsibility and Accountability

- Humanizing Self Identity and Sense of Community

- Consciousness of Economic, Social, Cultural, and Historical Life Barriers

- Inspiring Motivation \& Engagement

It is important to note that all 10 Participants discussed building Trust, High Expectations, and Caring Relationships as the foundation for teaching. Without trust and an established relationship with the students, the teachers noted that no learning would occur. In addition, they all emphasized the role of setting high expectations for student success.

Goal Oriented, Willingness to Act, and Perseverance was discussed as an important aspect pertaining to hope. Teachers understood that hope was more than just belief in something, 
EXAMINING TEACHERS' PARADIGM AND PEDAGOGY IN MANIFESTING CRITICAL HOPE IN LOW-INCOME MIDDLE SCHOOL

but that hope is connected to action and cycles of goal pursuits, the importance of setting a goal, and completing a goal. In addition, the teachers understood that failure is a part of the goal cycle and they addressed the need for students to look at failure as one more step closer to success.

Critical Reflection, Advocacy, Citizenry, and Empowerment was demonstrated by the teachers who truly understood the social, cultural, economic, political, and historical life barriers the students face on a daily basis. Consciousness of the life barriers caused awareness for the need to engage students in critical reflection, advocacy, citizenry, and empowerment as a way to overcome the hopelessness that these life barriers can cause in students.

On Valuing Optimism, the findings showed the emphasized importance the teachers demonstrated in connection to having an asset based, positive mindset, and hopeful outlook to combat the daily obstacles students in poverty face such as crime, trauma, depression, and hopelessness that is caused by these factors. The teachers felt like they had to be the hope and the positivity the students would need in order to be successful and to increase the hope and belief the students had for their own quest for success.

On Confronting Hopelessness, all 10 Participants noted the importance of sharing their own personal narratives in a way that is relevant and applicable to their students' lives. They expressed the importance of recognizing the models of excellence, which exist right in their own communities and that their stories needed to be told to raise the level of hope in students, so they can believe that success is possible. As Participant 4 stated, "Where you live doesn't define where you are going." In this way, using real-life examples are an important way to confront hopelessness in students of poverty.

Personal Commitment to Ethical Responsibility and Accountability was a big theme as teachers mentioned the need for teachers to take on the personal and ethical responsibility for 
students' success. If the students were not successful, then the accountability was placed upon the teachers. This is connected to hope because it demonstrates their belief in their students' abilities to succeed and the accountability placed on the educator to ensure students believe they can be successful and that they demonstrate that success in their classrooms daily.

Humanizing Self Identity and Sense of Community was also a big theme as teachers mentioned the importance of dialogue, valuing the students' experiences, and student voice. This theme was connected to sense of community, as it is a natural result of humanizing practices where teachers emphasized the need for community and sense of belonging in order for the dialogue to occur, which also connects to hope as in the ontological need for one's quest towards success through communal and humanizing practices (Freire, 1997).

Consciousness of Economic, Social, Cultural, and Historical Life Barriers is an imperative theme because teachers recognized what social cultural and economic barriers students face their daily in lives, what systemic oppressions are currently in place, and what historic oppressions are relevant to the lives of their students. This theme is what makes Critical Hope really critical as the awareness of the social, cultural, economic, political, and historical factors influence one's level of hope and it is the process of reflection and acting in ways that bring transformation of the world.

Inspiring Motivation and Engagement was a natural theme from the outpouring of hope and optimism teachers inhabited. Their belief in their students' success and their relentless expectations for students to see their capabilities and to believe in their future inspired a meaningful language and behavior, assignments, and lesson plans that provided multiple domains of engagement during instruction. All teachers noted the connection to hope and 
engagement by stating without hope, students would not be engaged as there would be no purpose to participate or to complete the task at hand.

\section{Recommendations}

Through the findings from the interviews of the 10 Participants as they used their authentic voices through narrative inquiry qualitative methodology, I am providing the following three recommendations for educators in order to make hope visible in the lives of students in low-income schools through professional development on Critical Hope.

\section{The first recommendation is for professional development on understanding hope} and its impact on student achievement: The what, the why, and the how. Critical Hope starts with an understanding of what hope is and what hope is not, and the why or the importance of increasing hope for student success. This professional development also shows how to implement pedagogy of Critical Hope in the classroom.

The second recommendation is for professional development on infusing hope in teachers' paradigm and pedagogy as an equity solution when working with students of poverty. This is important as my research findings support the notion that pedagogy and paradigm are inseparable, as one teaches in the manner that one believes. Teachers who had asset-based or hopeful paradigms also had language and behavior of hope, tasks of hope, and lesson planning of hope. Similarly, teachers who had a deficit-based or hopeless paradigm, had language and behavior of hopelessness, tasks of hopelessness, and lesson planning of hopelessness. This professional development also shows how teachers unintentionally create hopelessness through common teaching practices and beliefs that reflect hopeless paradigms, such as Symbolic Violence, Misrecognition, Unstable and Oppressive Environments, Self- 
EXAMINING TEACHERS' PARADIGM AND PEDAGOGY IN MANIFESTING CRITICAL HOPE IN LOW-INCOME MIDDLE SCHOOL

Deception, Narrative Sickness, and Self Depreciation ultimately impacting students' beliefs in their capabilities and their quest for success.

The third recommendation is for professional development on confronting hopelessness and embracing humanizing practices that increase students' identity for student achievement. This professional development shows how educators can confront hopelessness through authentic, relevant-personal narratives that demonstrate models of excellence that have been successful in their own communities despite all the out-of-school factors they face. In addition, when employing humanizing practices, students' belief in their own capabilities grow as they confront the hopelessness found in poverty through dialogue, reflection, and praxis which ultimately creates a sense of community in the classroom.

\section{From Freire (1994) to current formulations of Critical Hope}

Critical Hope paradigm-pedagogy that this research formulated is deduced from "powerful conclusions from a small set of abstract basic principles" (Gee, 2013, p. 52). Freire (1994) discussed the pedagogy of hope from a social and critical consciousness point of view describing how hope is an ontological need for humanity and without hope it is impossible to engage in the struggle. As previously stated, Freire (1994) states, "We need Critical Hope the way a fish needs unpolluted water" (p. 2). This demonstrates that without hope one is not really living, but rather becomes an object subjective to the dominating powers that control the world around them. Freire states that the discourse of those involved in the struggle must be "hopeful, critically optimistic, and drenched in ethics" (Freire, 1997, p. 43).

Duncan-Andrade (2009) views Critical Hope from a psychological perspective where he conceptualizes Critical Hope to describe three elements of educational practices that can build true hope in urban schools, namely, material hope, socratic hope, and audacious hope. Similarly, 
he discusses the three forms of false hope found in educational practices and beliefs: hokey hope, mythical hope, and hope deferred, which have decimated hope for children in urban schools and have been "an assault on hope, particularly in our nation's urban centers . . . including disinvestment in schools and overinvestment in prisons." (Duncan-Andrade, 2009, p. 182). He also notes that hope is inequitably dispersed and decimates along the gradient by social class (Duncan-Andrade, 2009).

The current dissertation deduced the notion of Critical Hope from the Critical Traditions of Educational Practices (CTEP) and the Hope Theory which includes, critical optimism, ethics, criticality, social consciousness, sense of community, caring relationships, knowledge of social and cultural capital, democratic advocacy, humanizing practices, goals, pathways, and agency. From data collected that manifests Critical Hope from teachers' paradigm and pedagogy, the current study provides visible evidence through the paradigm of hope that drives the language and behavior of hope, the instructional delivery of hope, and instructional planning of hope, which explicitly distinguishes and operationalizes Critical Hope through research and data. As such, Critical Hope has gone through the process of design, designing, and redesign and is defined as the optimistic way of viewing and acting on the world from a critically historically conscious, socially and culturally situated perspective with a personal belief that inevitable change will inspire a sense of community, advocacy, liberation, and justice. The following matrix shows the progression of Critical Hope that was initially introduced by Freire $(1994 ; 1997)$, built upon by Duncan-Andrade (2008; 2009), and redefined by Strikwerda (2019). 
Table 3

From Freire (1994;1997), to Duncan-Andrade (2008;2009), to Current Formulations of Critical Hope Based Upon Research Strikwerda (2019)

\begin{tabular}{|c|c|c|}
\hline $\begin{array}{c}\text { Freire } \\
(1994 ; 1997)\end{array}$ & $\begin{array}{c}\text { Duncan-Andrade } \\
(2008 ; 2009)\end{array}$ & $\begin{array}{c}\text { Strikwerda } \\
(2019)\end{array}$ \\
\hline $\begin{array}{l}\text { Critical Hope defined as a } \\
\text { new reading of my world } \\
\text { requires a "new language- } \\
\text { that of possibility, open to } \\
\text { hope" (Freire, 1997, p. 37). } \\
\text { This language of hope } \\
\text { includes a critical } \\
\text { consciousness of the } \\
\text { injustices in the world } \\
\text { expressed with words of } \\
\text { goodness and peace by } \\
\text { those who are engaged in } \\
\text { the struggle, and are in } \\
\text { search of permanent } \\
\text { possibilities that may } \\
\text { alleviate the inequities of } \\
\text { the oppressed (Freire, } \\
\text { 1997). }\end{array}$ & $\begin{array}{l}\text { Critical Hope defined by } \\
\text { hooks (1994 as cited in } \\
\text { Duncan-Andrade, 2008) "the } \\
\text { world can be a place opposed } \\
\text { to domination and oppression } \\
\text { and that Critical education can } \\
\text { trigger all people, privileged } \\
\text { and oppressed, to act in ways } \\
\text { that liberate ourselves and } \\
\text { others" (p. 37). }\end{array}$ & $\begin{array}{l}\text { Critical Hope is defined as the } \\
\text { optimistic way of viewing and } \\
\text { acting in the world from a } \\
\text { critically, historically } \\
\text { conscious, socially, and } \\
\text { culturally situated perspective } \\
\text { with a personal belief that } \\
\text { inevitable change will inspire } \\
\text { a sense of community, } \\
\text { advocacy, liberation, and } \\
\text { justice. }\end{array}$ \\
\hline
\end{tabular}

\section{Conclusion}

As we have seen, there are many out-of-school factors that may have an impact on student achievement (Berliner, 2005). Systemic oppression is a result of inequitable paradigms that drive policies and institutionalized practices within society, which ultimately is internalized by educators and the students who constantly face these inequities. Internalized oppression can lead to waves of horizontal violence, and an acceptance of their place in this world (Freire, 1970) preventing students from believing that they can break out of the poverty cycle. Thus, educators continue the cycle of oppressive common practices, which creates hopeless paradigm-pedagogy by distributing acts of unintentional hopelessness through symbolic violence, misrecognition, 
oppressive environments, self-deflection, self-depreciation, and narrative sickness. According to these research findings, all of these tendencies impact the level of hope in educators and students of poverty creating what I have coined to be the hope gap, or the zone of difference, representing sets of expressions and actions that reflect hope and hopelessness in humanity. My question then becomes: Are the invisible factors of systemic oppression, internalized oppression, unintentional hopelessness and the hope gap, these factors that cannot be seen on high-stakes test scores or achievement scores, related to the visible and continual achievement gap that exists between low-income students and affluent students?

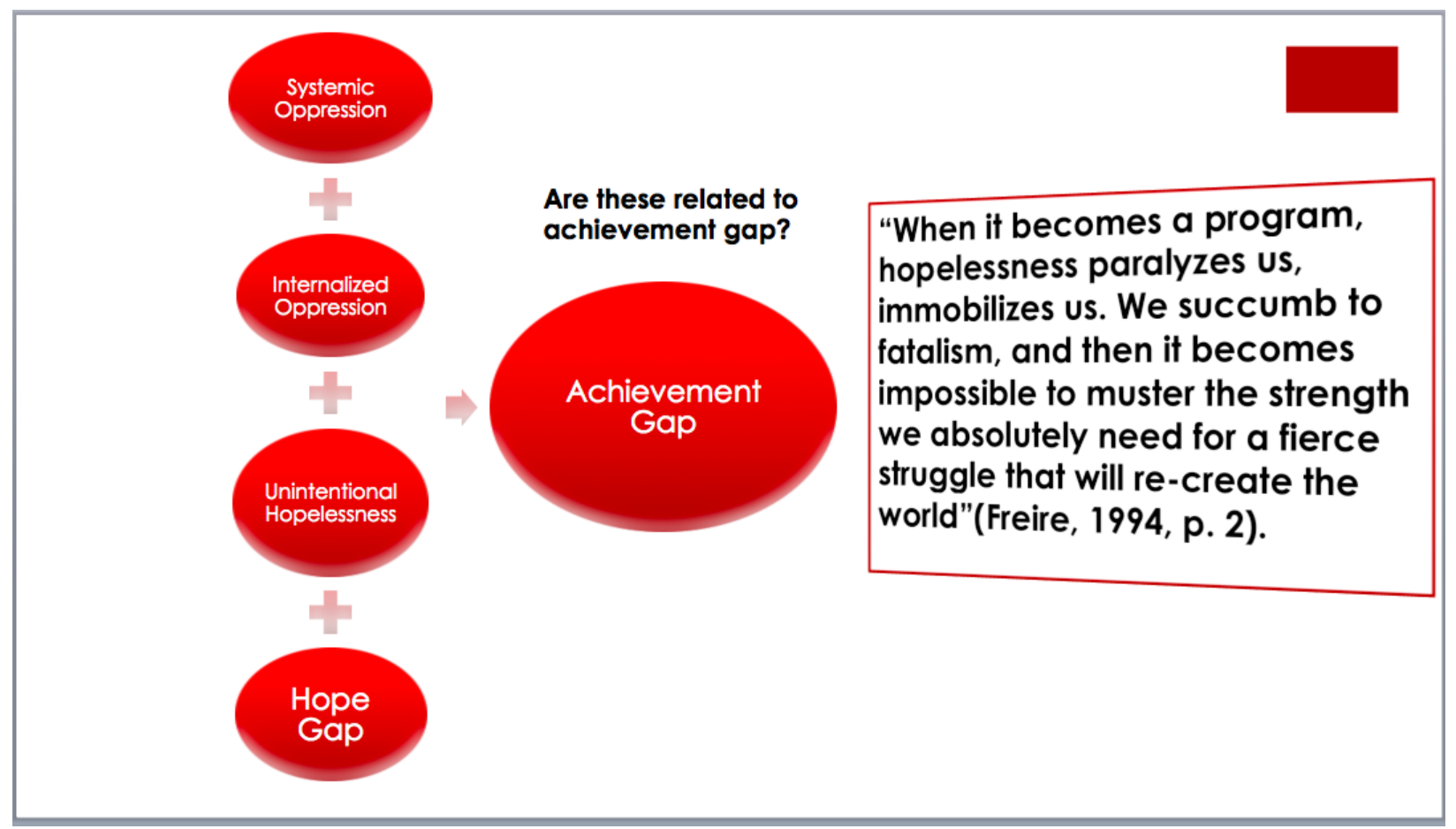

Figure 52: Invisible Factors that May Relate to the Continual and Existing Achievement Gap between Affluent Students and Low-Income Students

As previously stated, without hope one cannot begin to engage in the struggle (Freire, 1994). Thus, educators of children of poverty need to confront hopelessness through equitable, 
humanizing practices that move humanity beyond resistance and towards transformation through Critical Hope, love, and justice. "One of the tasks of the progressive educator, through a serious, correct political analysis, is to unveil opportunities for hope, no matter what obstacles may be" (Freire, 1994, p. 3). This research has added to the knowledge of Critical Hope by starting from the idea of Critical Hope and making Critical Hope tangible and visible through evidence that emerged from the voices of teachers, which can be pragmatically taught and applied inside the classrooms as an equity solution for poverty schools. Let us not be people who collapse under the weight and weariness found in the obstacles, but rather let us be those educators who are equity centered, removing barriers, raising hope, and moving beyond resistance towards transformation. As Dr. Martin Luther King states, "Out of a mountain of despair, a stone of hope" (Yorder, 2018, p. 14)

\section{Limitations}

Since I was not given IRB approval to observe student engagement, I was not able to make any conclusions on how Critical Hope impacts or directly influences student engagement. Thus, all references towards students in relation to manifestations of Critical Hope are inferred in the perspective and view of the teachers and have not been expressed by the students themselves.

\section{Future Research}

Future research is needed to gain an awareness of the direct connection between the themes and elements of Critical Hope and the multidimensional construct of student engagement. In order to identify student engagement in relation to Critical Hope paradigm-pedagogy, students would need to be observed and interviewed, data would need to be collected and analyzed, and findings would need to be discussed. How students see themselves in relation to the two positions of hope and hopelessness has an impact on student achievement (Edwards et al., 2007). 
Research demonstrated that motivation and engagement are directly connected to student achievement, as motivation is the internal intent while engagement is the output or the action (Bingham \& Okagaki, 2012, Cooper, 2014; Reeve, 2012). In order for students to be engaged, they must first be motivated. Motivation is an internalized drive that is sensitive to the opportunities provided to students in experiencing competence, autonomy, meaningfulness, and sense of belonging and research showed that students who are given the opportunity to experience motivation in these ways have higher student engagement and academic achievement (Lalas, Charest, Strikwerda, \& Ordaz, 2019). Engagement is a multidimensional construct consisting of behavioral engagement, cognitive engagement, affective engagement, and agentic engagement and is directly connected to student learning (Reeve 2012; Turner et al., 2014). The need for future research would be in the direct connection in how Critical Hope paradigmpedagogy influences motivation and student engagement.

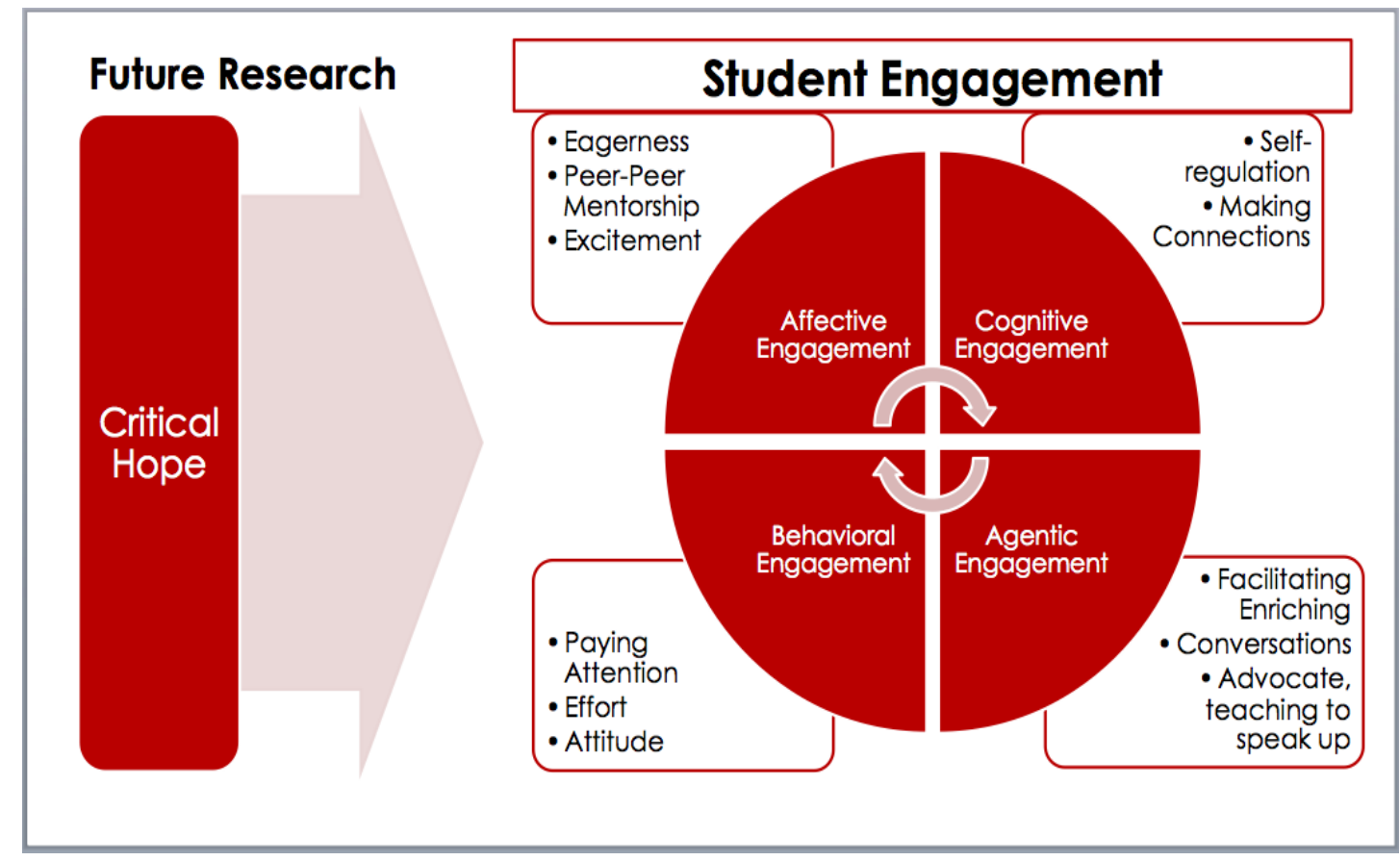

Figure 53: Critical Hope and Its Influence on Students Engagement 
EXAMINING TEACHERS' PARADIGM AND PEDAGOGY IN MANIFESTING CRITICAL HOPE IN LOW-INCOME MIDDLE SCHOOL

\section{References}

Althusser, L. (1970). Ideology and ideological state apparatuses. Lenin and philosophy and other essays. Retrieved from https://www.marxists.org/reference/archive/althusser/ 1970/ideology.htm

Anyon, J. (1980). Social class and the hidden curriculum of work. The Journal of Education, 162(1), 67-92. Retrieved from http://www.jstor/stable/42741976

Anyon, J. (2005). What counts as educational policy? Notes toward a new paradigm. Harvard Educational Review, 75(1), 65-68.

Anyon, J. (2011). Marx and education. New York, NY: Routled ge. doi:10.4324/9780203829615

Bakhtin, M. M. (1981). The dialogue of imagination: Four essays. Austin, TX: University of Texas Press.

Bell, D. (1980). Brown v. Board of Education and the interest-convergence dilemma. Harvard Law Review, 93, 513.

Bell, L. (2007). Theoretical foundations for social justice education: Teaching for diversity and social justice. New York: NY: Routledge.

Berliner, D. (2005). Our impoverished view of education. Teachers College Press, 1-63.

Retrieved from https://nepc.colorado.edu/sites/default/files/EPSL-0508-116EPRU\%5B1\%5D.pdf

Biddle, B. J., \& Berliner, B. D. (2002). A research Synthesis: Unequal school funding in the United States. Educational Leadership, 59(8), 48-59. Retrieved from http://www.ascd.org/publications/educational-leadership/may02/vol59/num08/UnequalSchool-Funding-in-the-United-States.aspx 
Bingham, G. E., \& Okagaki, L. (2012). Ethnicity and student engagement. In S. L. Christenson, A. L. Reschly, \& D. Wylie (Eds.), Handbook of research on student engagement (pp. 6595). Springer Science + Business Media.

Bonilla-Silva, E. (2017). Racism without racists: Color-blind racism and the persistence of racial inequality in America (5th ed.). New York NY: Rowman \& Littlefield Publishing. Boulding, K. (1972). The schooling industry as a possible pathological section of the American economy. Review of Educational Research, 42(1), 129-143.

Bourdieu, P. (1986). The forms of capital. In J. Richardson (Ed.). Handbook of theory and research for the sociology of education, (241-258). Westport, CT: Greenwood.

Bourdieu, P. (1991). Language and symbolic power. Trans. Gino Raymond and Matthew Adamson. Cambridge, MA: Harvard University Press.

Bourdieu, P. (1998). Acts of resistance against the tyranny of the market. New York, NY: The New Press.

Bourdieu, P. (2005). The social structures of the economy. UK: Polity Press.

Brookfield, S. (2005). The power of critical theory for adult learning and teaching. New York, NY: Open University Press.

Brown v. Board of Education. (1954). Brown v. Board of Education of Topeka, 347 U.S. 483 (1954). Retrieved from https://supreme.justia.com/cases/federal/us/347/483/case.html California Department of Education. (2016). Understanding your students score report: Grades 4, 6, \& 7. Retrieved from https://tb2cdn.schoolwebmasters.com/accnt_13143/site_13144/ Documents/UnderstandingCAASPPGr467_Upland_062916.pdf 
EXAMINING TEACHERS' PARADIGM AND PEDAGOGY IN MANIFESTING CRITICAL HOPE IN LOW-INCOME MIDDLE SCHOOL

Chen, T., \& Hahnel, C. (2017, April). The steep road to resource equity in California education. Education Trust-West, 1-19. Retrieved from https://west.edtrust.org/wp-content/ uploads/sites/3/2015/11/ETW_Steep-Road-to-Resource-Equity-in-CA_Final_Report _April_11_2017.pdf

Clayton, V. (2016, June 30). Turns out common core isn't about politics, it's about social justice [Web log post]. Retrieved from https://educationpost.org/turns-out-common-core-isntabout-politics-its-about-social-justice/

Cochran-Smith, M., Ell, F., Grundoff, L., Haigh, M., Hill, M., \& Ludlow. (2016). Initial teacher education: What does it take to put equity at the center? Teaching and Teacher Education, $57,67-78$

Coleman, J. S., Campbell, E. Q., Hobson, C. J., McPartland, J., Mood, A. M., Weinfeld, F. D., \& York, R. L. (1966). Equality of educational opportunity. Washington, DC: U. S. Government Printing Office.

Conle, C. (2001). The rationality of narrative inquiry in research and professional development. European Journal of Teacher Education, 24(1), 21-33.

Conle, C. (2006). Images of change in narrative inquiry. Teachers and Teaching, 2(2), 205-219. Retrieved from https://www.researchgate.net/publication/233151913_Images_of_ Change_in_Narrative_Inquiry

Connelly, F. M., \& Clandinin, D. J. (1990). Stories of experience and narrative inquiry. Educational Researcher, 19(5), 2-14.

Cooley, A. (2018). War on poverty: United States history. Encyclopedia Britannica. Retrieved from https://www.britannica.com/topic/War-on-Poverty 
EXAMINING TEACHERS' PARADIGM AND PEDAGOGY IN MANIFESTING CRITICAL HOPE IN LOW-INCOME MIDDLE SCHOOL

Cooper. K. (2014). Eliciting engagement in the high school classroom: A mixed-methods examination of teaching practices. American Education Research Journal, 51(2), 363402.

Crave, A. (2018). California ranked 42nd nationally in education. Retrieved from https://www.abc10.com/article/news/local/california/california-ranked-42nd-nationallyin-education/387174912

Creswell, J., \& Poth, C. (2018). Qualitative inquiry and research design: Choosing among five approaches (4th ed.). Thousand Oaks, CA: Sage Publications.

Darder, A. (2009). The critical pedagogy reader. New York, NY: Routledge.

Darling-Hammond, L., Amrein-Bead sley, A., Haertel, E., \& Rothstein, J. (2012). Evaluating teacher evaluation. Phi Delta Kappan, 93(6), 8-15. doi:10.1177/003172171209300603

Darling-Hammond, L., Bae, S., Cook-Harvey, C. M., Lam, L., Mercer, C., Podolsky, A., \& Stosich, E. L. (2016). Pathways to new accountability through the Every Student Succeeds Act. Retrieved from https://learningpolicyinstitute.org/sites/default/files/ product-files/Pathways_New-Accountability_Through_Every_Student_Succeeds_ Act_04202016.pdf

Darling-Hammond, L., \& Post, L. (2000). Inequality in teaching and schooling: Supporting highquality teaching and leadership in low-income schools. In R. D. Kahlenberg (Ed.), $A$ nation at risk: Preserving public education as an engine for social mobility (pp. 127167). New York, NY: The Century Foundation Press.

Deci, E., \& Ryan, R. (2015). Self-determination theory. Retrieved from http://duwtje.com/wpcontent/uploads/2015/06/Self-Determination-Theory.pdf 
EXAMINING TEACHERS' PARADIGM AND PEDAGOGY IN MANIFESTING CRITICAL HOPE IN LOW-INCOME MIDDLE SCHOOL

Delgado, R., \& Stefancic, J. (2017). Critical race theory: An introduction. New York, NY: New York University Press.

Dettling, L. J., Hsu, J. W., Jacobs, L., Moore, K. B., \& Thompson, J. P. (with Lianes, E.). (2017). Recent trends in wealth-holding by race and ethnicity: Evidence from the survey of customer finances. FEDS Notes. Retrieved from https:/www.federalreserve.gov/econres/ notes/feds-notes/recent-trends-in-wealth-holding-by-race-and-ethnicity-evidence-fromthe-survey-of-consumer-finances-20170927.htmFigure 1)

Dewey, J. (1938). Experience and education. New York, NY: Kappa Delta Pi Publications.

Duncan-Andrade, J. (2009). Note to educators: Hope required when growing roses in concrete. Harvard Educational Review, 79(2), 181-194.

Duncan-Andrade, J., \& Morrell, E. (2008). The art of critical pedagogy: Possibilities for moving from theory to practice in urban schools. New York, NY: Peter Lang Publishing.

Education Trust-West. (2017). The majority report: Supporting the educational success of Latino students on California. Retrieved from https://west.edtrust.org/wp-content/uploads/sites /3/2017/11/ETW_TMR_FINAL.pdf

Education Week Research Center. (2018). Quality counts 2018: Report and rankings: A report card for states and the Nation on K-12 Education. Retrieved from https://www.edweek. org/ew/collections/quality-counts-2018-state-grades/index.html

Edwards, L. M., Rand, K. L., Lopez, S. J., \& Snyder, C. R. (2007). Understanding hope: A review of measurement and construct validity research. In A. D. Ong, M. H. M. van Dulmen (Eds.), Oxford handbook of methods in positive psychology, (83-95). New York, NY: Oxford University Press. 
EXAMINING TEACHERS' PARADIGM AND PEDAGOGY IN MANIFESTING CRITICAL HOPE IN LOW-INCOME MIDDLE SCHOOL

Elementary and Secondary Education Act of 1965 (ESEA). (1965). Elementary and Secondary Education Act of 1965. Retrieved from https:/www2.ed.gov/about/offices/list/oii/ nonpublic/eseareauth.pdf

Every Student Succeeds Act. (2015). Public Law 114-95. Retrieved from https://www.congress. gov/114/plaws/pub195/PLAW-114pub195.pdf

Elliott, M. (1998). School finance and opportunity to learn: Does money well spent enhance students' achievement? Sociology of Education, 71(3), 223-245.

Etikan, I., Musa, S. A., \& Alkassim, R. S. (2016). Comparison of convenience sampling and purposive sampling. American Journal of Theoretical and Applied Statistics, 5(1), 1-4.

Felter, J., \& Ayers, L. (2016). Incorporating trauma informed practice and ACEs into professional curricula - A toolkit. The Philadelphia ACE Project. Retrieved from http://mind peacecincinnati.com/wp-content/uploads/Incorporating-Trauma-InformedPractice-and-ACEs-into-Professional-Curricula-a-Toolkit.pdf

Ferguson, R. F. (1991). Paying for public education: New evidence on how and why money matters. Harvard Journal on Legislation, 28(2), 465-498.

Ferguson, R. F., \& Ladd, H. F. (1996). How and why money matters: An analysis of Alabama schools. In H. F. Ladd(Ed.), Holding schools accountable: Performance-based reform in education (pp. 265-298). Washington, DC: The Brookings Institution.

Finn, J. D., \& Zimmer, K. S. (2012). Student engagement: What is it? Why does it matter? In S. L. Christenson, A. L. Reschly, \& C. Wylie (Eds.), Handbook of research on student engagement (pp. 97-131). New York, NY: Springer.

Flax, J. (1998). American dream in Black and White: The Clarence Thomas hearings. Ithaca, NY: Cornell University Press. 
EXAMINING TEACHERS' PARADIGM AND PEDAGOGY IN MANIFESTING CRITICAL HOPE IN LOW-INCOME MIDDLE SCHOOL

Flint, T. (2018). The price of inequality: The achievement gap and the high cost to America's future. California Schools Quarterly Publication of the California School Boards Association, 77(1), 22-33.

Flores, B., Strikwerda, H., \& Ordaz, M. (2019). A comparative paradigmatic matrix of EL policy, ideology, pedagogy, and practices. Multilingual Educator: CABE. Retrieved from http://www.gocabe.org/wp-content/uploads/2019/04/CABE2019ME_FINAL.pdf

Fontenot, K, Semega, J, \& Kollar, M. (2018). Income and poverty in the United States: 2017. Current population reports. Washington, DC: U.S. Government Printing Office. Retrieved from https://www.census.gov/content/dam/Census/library/publications/ 2018/demo/p60263.pdf

Fox, L. (2017). The supplemental poverty measure: 2016: Current Population Reports. Retrieved from https://www.census.gov/content/dam/Census/library/publications/ 2017/demo/p60-261.pdf

Fraser, N. (1997). Justus Interuptus: Critical reflections on the "postsocialist" condition. New York, NY: Routledge.

Fredricks, J. A., Blumenfeld, P. C., \& Paris, A. H. (2004). School engagement: Potential of the concept, state of the evidence. Review of Educational Research, 74(1), 59-109

Freire, P. (1970). Pedagogy of the oppressed. New York, NY: Continuum.

Freire, P. (1974). Education for critical consciousness. London: Sheed and Ward Ltd.

Freire, P. (1994). The pedagogy of hope. New York, NY: Continuum.

Freire, P. (1997), Pedagogy of the heart. New York, NY: Continuum.

Freire, P., \& Macedo, D. (1987). Literacy: Reading the word and the world. New York, NY: Continuum. doi:https://doi.org/10.4324/9780203986103 
Gay, G. (2000). Culturally responsive teaching. New York, NY: Teachers College Press.

Gay, G. (2002). Preparing for culturally responsive teaching. Journal of Teacher Education, 53(2), 106-116.

Gee, J. P. (2013). Discourses in and out of school: Looking back. In M. Hawkins (Ed.), Framing languages and literacies: Socially situated views and perspectives (pp. 51-83). New York, NY: Routledge. doi:10.4324/9780203070895

Geertz, C. (1973). The interpretation of culture. New York, NY: Basic Books.

Giroux, H. (1983). Theory and resistance in education. South Hadley, MA: Bergin \& Garvey.

Giroux, H. (1997). Pedagogy and the politics of hope: Theory, culture, and schooling, a critical reader. Boulder, CO: Westview Press.

Giroux, H. (2011). Critical pedagogy. New York, NY: Bloomsbury.

González, N., Moll, L. C., \& Amanti, C. (Eds.). (2005). Funds of knowledge: Theorizing practices in households, communities, and classrooms. Mahwah, NJ: Lawrence Erlbaum Associates.

Greenwald, R., Hedges, L. V., \& Laine, R. D. (1996). The effect of school resources on school achievement. Review of Educational Research, 66(3), 361-396.

Habermas, J. (1984). The theory of communicative action: Reason and the rationalization of society (Volume One). Boston, MA: Beacon Press.

Hakuta, K. (2017). California English learner roadmap. Strengthening comprehensive educational policies, programs, and practices for English learners. Sacramento, CA: California Department of Education. Retrieved from http:/cabe2018.gocabe.org/wpcontent/uploads/2018/03/CDE_EL_Roadmap_Guidance.pdf 
Hansen, C. (2018). States with the highest poverty rates. Retrieved from https://www.usnews. com/news/best-states/slideshows/states-with-the-highest-poverty-rates-in-the-us

Hanushek, E. A. (1989). The impact of differential expenditures on school performance. Educational Researcher, 18(4), 45-65.

Hanushek, E. A. (1996). School resources and student performance. In G. Burtless (Ed.), Does money matter? The effect of school resources on student achievement and adult success (pp. 43-73). Washington, DC: The Brookings Institution.

Harmon, D. (2012). Culturally responsive teaching through a historical lens: Will history repeat itself? Interdisciplinary Journal of Teaching and Learning, (2)1, 1-22.

Hawkins, M. (2014). Framing languages and literacies: Socially Situated Views and Perspectives. Journal of Language Identity and Education, 13(5), 192-213.

Hedges, L. V., \& Greenwald, R. (1996). Have times changed? The relation between school resources and student performance. In G. Burtless (Ed.), Does money matter? The effect of school resources on student achievement and adult success (pp. 74-92). Washington, DC: The Brookings Institution.

Hedges, L. V., Laine, R. D., \& Greenwald, R. (1994). Does money matter? A meta-analysis of studies of the effects of differential school inputs on student outcomes. Educational Researcher, 23(3), 5-14.

hooks, bell. (2003). Teaching community: A pedagogy of hope. New York, NY: Routledge. Howard, T. (2010). Why race and culture matter in schools. New York, NY: Teachers College Press. 
EXAMINING TEACHERS' PARADIGM AND PEDAGOGY IN MANIFESTING CRITICAL HOPE IN LOW-INCOME MIDDLE SCHOOL

Jean-Marie, G., Normore, A. H., \& Brooks, J. S. (2009). Leadership for social justice: Preparing 21st century school leaders for a new social order. Journal of Research on Leadership Education, 4, 1-31.

Johnson, A. G. (2007). Privilege, power, and difference. New York, NY: McGraw-Hill.

Kalantzis, M., Cope, B., Chan, E., \& Dally-Trim, L. (2016) Literacies. Sydney: Cambridge University Press.

Kozol, J. (2007). The shame of a nation: The restoration of Apartheid schooling in America. New York, NY: Three Rivers Press.

Kuhn, T. (1962). The structure of scientific revolutions. Chicago, IL: The University of Chicago Press.

Kumashiro, K. K. (2015). Against common sense: Teaching and learning toward social justice (3rd ed.). New York, NY: Routledge. doi:10.4324/9781315765525

Ladson-Billings, G. (1994). The dream keepers: Successful teachers of African American children. San Francisco, CA: Jossey-Bass.

Ladson-Billings, G. (2005). Culturally relevant teaching: the key to making multicultural education work. In C. A. Grant (Ed.), Research and multicultural education: From the margins to the mainstream (102-118). Bristol, PA: Falmer Press.

Ladson-Billings, G. (2013). What critical race theory is not! In Lynn, M., \& A. D. Dixson (Eds.), Handbook of critical race theory in education (pp. 34-47). New York, NY: Routledge.

Ladson-Billings, G. (2014). Culturally relevant pedagogy 2.0: a.k.a. the remix. Harvard Educational Review, 84(1), 74-84. Retrieved from http://piggottsclass.weebly.com/ uploads/2/3/1/7/23179512/ladson-billings_culturally_relevant_pedagogy.pdf 
EXAMINING TEACHERS' PARADIGM AND PEDAGOGY IN MANIFESTING CRITICAL HOPE IN LOW-INCOME MIDDLE SCHOOL

Ladson-Billings, G., \& Tate, W. (1995). Toward a critical race theory of education. Teachers College Record, 97(1), 47-68.

Lalas, J. (2007). Teaching for social justice in multicultural urban schools: Conceptualization and classroom implication. Multicultural Education, 14(3), 17-21.

Lalas, J., Charest, B., Strikwerda, H., \& Ordaz, M. (2019). Nurturing hope, sense of belonging and engagement through equity. In C. Forlin (Sen. Ed.), Promoting social inclusion: Cocreating environments that foster equity, hope and belonging (Vol. 13; pp. 41-52). New Zealand; Emerald.

Lalas, J., Macias, A., Fortner, K., Flores, N., Blackmon-Balogun, \& Vance, M. (2016). Who we are and how we learn: Educational engagement and justice for diverse learners. San Diego, CA: Cognella Academic Publishing.

Lopez, S. (2013). Making hope happen: Create the future you want for yourself and others. New York, NY: Atria

Luschei, T. F., \& Jeong, D. W. (2018). Is teacher sorting a global phenomenon? Cross-national evidence on the nature and correlates of teacher quality opportunity gaps. $A E R A, 47(9)$, $556-576$.

Macias, A., \& Lalas, J. (2014). Funds of knowledge and student engagement: A qualitative study on Latino high school students. Learning Landscapes, 7(2), 195-217.

Mahatmya, D., Lohman, B. J., Matjasko, J. L., \& Farb, A. F. (2012). Engagement across developmental periods. Retrieved from https://ib.dr.iastate.edu/cgi/viewcontent. cgi? article $=1023 \&$ context $=$ hdfs_pubs

Marx, K., \& Engels, F. (1848). Manifesto of the Communist Party. Selected Works (Volume One). Moscow: Progress Publishers. Retrieved from https://www.marxists.org/archive/ 
marx/works/download/pdf/Manifesto.pdf

Maslow, A. H. (1943). A theory of human motivation. Psychological Review, 50(4), 370-396.

Mason, C. N. (2017). The American Dream: Only 4\% of America's poor rise to the middle. What causes poverty? Not a lack of money, but a lack of social relationships. Retrieved from http://cnicolemason.com/index/uncategorized/346/

McKee, G. A. (2010). Lyndon B. Johnson and the War on Poverty. Retrieved from https://prde.upress.virginia.edu/content/WarOnPoverty

McLaren, P. (1994). Life in schools: An introduction to critical pedagogy and the foundations of education. New York, NY: Longman.

McLaren, P. (2003). Life in schools: An introduction to critical pedagogy in the foundations of education (2nd ed.). Boston, MA: Allyn and Bacon.

Misra. T. (2015). The stark inequality of U.S. public schools, mapped. Retrieved from https://www.citylab.com/equity/2015/05/the-stark-inequality-of-u s-public-schools$\underline{\text { mapped/393095/ }}$

Moll, L. C., \& Gonzales, N. (2004). Engaging life: A funds of knowledge approach to multicultural education. In J. A. Banks \& C. A. M. Banks (Eds.), Handbook of research on multicultural education (pp. 699-715). New York, NY: Jossey-Bass.

Murphy, M., \& Fleming, T. (2010). Taking aim at the heart pf education: Critical theory and the future of learning. In M. Murphy \& T. Fleming, T. (Eds.), Habermas, critical theory and education (pp. 201-207). Routledge International Studies in the Philosophy of Education. New York, NY: Routledge.

N., P. (2013). Cultural blindness. Retrieved from https://psychologydictionary.org/culturalblindness/ 
Neuberger, N., \& Riddle, W. (2015). How to identify low-income students in "community eligibility" schools for Title I purposes. Retrieved from https://www.cbpp.org/sites/default/files/atoms/files/6-2-14fa.pdf

Niemiec, C. P., \& Ryan, R. M. (2009). Autonomy, competence, and the relationship in the classroom: Applying self-determination theory to educational practice. Theory and Research in Education, 7(2), 133-144. doi:10.1177/1477878509104318

No Child Left Behind Act of 2001. (2002). Public Law 107-110: An Act to close the achievement gap with accountability, flexibility, and choice, so that no child is left behind. Retrieved from http://www2.ed.gov/policy/elsec/leg/esea02/107-110.pdf

Noddings N. (2017). Care ethics and education. In N. Aloni, \& L. Weintrob (Eds), Beyond Bystanders: Educational leadership for a humane culture in a globalizing reality (pp. 183-190). Rotterdam, The Netherlands: Sense Publishers.

North, C. (2008). What is all this talk about social justice? Teacher College Record, 110(6). 1182-1206.

Opitz, B., Ferdinand, N. K., \& Mecklinger, A. (2011). Timing matters: The impact of immediate and delayed feedback on artificial language learning. Frontiers in Human Neuroscience, 5(8). doi:10.3389/fnhum.2011.00008

Orshansky, M. (1965). Who's who amongst the poor: A demographic view of poverty. Retrieved from https://pdfs.semanticscholar.org/50ce/69e1504390beb21af3c30ac5a3db7177 58a0.pdf

Owen, S., \& Sawhill, I. (2013). Should everyone go to college? Center on children and Families at BROOKINGS, CCF Brief\#50. Retrieved from https://www.brookings.edu/wpcontent/up loads/2016/06/08-should-everyone-go-to-college-owen-sawhill.pdf 
EXAMINING TEACHERS' PARADIGM AND PEDAGOGY IN MANIFESTING CRITICAL HOPE IN LOW-INCOME MIDDLE SCHOOL

Patton, M. (2002). Qualitative research \& evaluation methods. Thousand Oaks, CA: Sage Publications.

Peter G. Peterson Foundation. (2018, September 13). Income and wealth in the United States: An overview of recent data [Web log post]. Retrieved from https:/www.pgpf.org/blog/2018/ 09/income-and-wealth-in-the-united-states-an-overview-of-data

Pike, G. R. (2002). Measurement issues in outcomes assessment. In T. W. Banta \& Associates, Building a scholarship of assessment (pp. 131-147). San Francisco, CA: Jossey-Bass.

Poverty. (2017). How the census bureau measures poverty. Retrieved from https://www.census. gov/topics/income-poverty/poverty/guidance/poverty-measures.html

Rawlinson. R. (2011). A mind shaped by poverty: 10 things educators should know. Bloomington, IN: Universe.

Rector, R., \& Sheffield, R. (2011). Air conditioning, cable tv, and an xbox: What is poverty in the United States today? The Heritage Foundation, No. 2575, 1-25. Retrieved from http://thf_media.s3.amazonaws.com/2011/pdf/bg2575.pdf

Reeve, J. (2012). A self-determination theory perspective on student engagement. In S. L. Christenson, A. L. Reschly, \& C. Wylie (Eds.), Handbook of research on student engagement (pp. 149-171). New York, NY: Springer Science + Business Media.

Renwick, T., Short, K., Bishaw, A., \& Hokayem, C. (2012). Using the American community survey (ACS) to implement a supplemental poverty measure (SPM). SEHSD Working Paper \#2012-10. Retrieved from https://www.census.gov/content/dam/Census/library/ working-papers/2012/demo/SEHSD-WP2012-10.pdf

Reschly, A. L., \& Christenson, S. L. (2012). Jingle, jangle, and conceptual haziness: Evaluation of future directions of the engagement construct. In S. L. Christenson, A. L. Reschly, \& 
EXAMINING TEACHERS' PARADIGM AND PEDAGOGY IN MANIFESTING CRITICAL HOPE IN LOW-INCOME MIDDLE SCHOOL

C. Wylie (Eds.), Handbook of research on student engagement (pp. 3-19). New York, NY: Springer.

Ryan, R., \& Weinstein, N. (2009). Undermining quality teaching and learning: A selfdetermination theory perspective on high-stakes testing. Theory and Research in Education, 7(2) 224-233

Ryan, W. (1971). Blaming the victim. New York, NY: Random House.

Saltman, K. (2018). The politics of education: A critical introduction. New York, NY: Routledge.

Schmoker, M. (2012). Refocus professional development. Phi Delta Kappan, 93(6), 68-69.

Schneiderman, H. G. (2017). Engagement \& disengagement: Class, authority, politics, and intellectuals. New York, NY: Routledge. doi:https://doi.org/10.4324/9781315100074

Seidman, I. (2006). Interviewing as qualitative research: A guide for researchers in education and the social sciences (3rd ed.). New York, NY: Teachers College Press

Senge, P. (2012). Schools that learn. New York, NY: Crown Business.

Sensoy, O., \& DiAngelo, R. (2012). Is everyone really equal? New York, NY: Teachers College Press.

Short, K. (2011). The research supplemental poverty measure: 2010: Consumer income. Retrieved from https://www.census.gov/prod/2011pubs/p60-241.pdf

Shuey, A. (1966). The testing of Negro intelligence. Lynchburg, VA: Social Science Press.

Shulman, L. (2007). Counting and assessment and the quest for accountability. Change: The Magazine of Higher Learning, 39(1), 20-25.

Skinner, E., \& Pitzer, J. (2012). Developmental dynamics of student engagement, coping, and everyday resilience. New York, NY: Spinner Science + Business Media. 
EXAMINING TEACHERS' PARADIGM AND PEDAGOGY IN MANIFESTING CRITICAL HOPE IN LOW-INCOME MIDDLE SCHOOL

Spellings, M. (2005). 10 Facts about K-12 educational funding. Washington, DC: U. S. Department of Education.

Steele, C. M., \& Aronson, J. (1995). Stereotype threat and the intellectual test performance of African Americans. Journal of Personality and Social Psychology, 69(5), 797-811

Swartz, D., (1997). Bourdieu's political economy of symbolic power. In D. Swartz (Ed.), Culture and power: The sociology of Pierre Bourdieu (pp. 65-94). Chicago, IL: The University of Chicago Press.

Terada, Y. (2019). Understanding a teacher's long-term impact. Retrieved from https://www.edutopia.org/article/understanding-teachers-long-term-impact

Theoharis, G. (2007). Social justice educational leaders and resistance: Toward a theory of social justice leadership. Education Administration Quarterly, 43(2), 221-258.

Turner, J. C., Christenson, A., Kackar-Cam, H. Z., Trucano, M., \& Fulmer, S. M. (2014). Enhancing students' engagement: Report of a 3-year intervention with middle school teachers. American Educational Research Journal, 51(6), 1195-1226. doi:10.3102/000 2831214532515

Ullucci, K., \& Howard, T. (2015). Pathologizing the poor: Implications for preparing teachers to work in high poverty schools. Urban Education, 50(2), 170-193.

U. S. Census Bureau (2018). Historical poverty tables: People and families-1959-2017. Retrieved from https://www.census.gov/data/tables/time-series/demo/income-poverty/ historical-poverty-people.html.

Valencia, R. (1997). The evolution of deficit thinking: Educational thought and practice. contemporary deficit thinking. New York, NY: Routledge Falmer. 
Webb, L. D., \& Norton, M. S. (2009). Human resources administration. (5th ed.). Upper Saddle River, NJ: Merrill.

Woolley, III, J. T., \& Peters, G. (n.d.). Lyndon B. Johnson: Annual message to the Congress on the state of the union on January 8, 1964. Retrieved from http://www.presidency.ucsb.edu/ ws/index.php?pid=26787

Yorder, M. (2018). Out of a mountain of despair a stone of hope: MLK memorial brings famous speech to life. Retrieved from https://www.wusa9.com/article/news/nation-world/out-ofa-mountain-of-despair-a-stone-of-hope-mlk-memorial-brings-famous-speech-to-life/507534376186

Zhou, M. M., \& Kam, C. (2016): Hope and general self-efficacy: Two measures of the same construct? The Journal of Psychology, 150 (5), 543-559. doi:10.1080/00223980.2015. 1113495 
EXAMINING TEACHERS' PARADIGM AND PEDAGOGY IN MANIFESTING CRITICAL HOPE IN LOW-INCOME MIDDLE SCHOOL

\section{Appendix A}

\section{Participant 1: Interview and Observation}

\begin{tabular}{|c|c|c|}
\hline $\begin{array}{l}\text { Participant } \\
\text { P.1 5/23/19 }\end{array}$ & Questions & Comments \\
\hline Teacher & $\begin{array}{l}\text { Introduction: Thank you for } \\
\text { a greeing to participate in my study on } \\
\text { Examining Teachers' Paradigm and } \\
\text { Pedagogy in Manifesting Critical Hope in } \\
\text { Low-Income Middle School Classrooms: A } \\
\text { Narrative Inquiry } \\
\text { This interview will last } \\
\text { approximately } 30 \text { to } 45 \text { minutes. I will be } \\
\text { a sking you to review the transcript of the } \\
\text { interview for accuracy. } \\
\qquad 1 \text {. (PI's introduction to questions) } \\
\text { START WITH an OPEN-ENDED } \\
\text { QUESTION: JUST LIKE TELLING A } \\
\text { STORY: We a lready know through existing } \\
\text { research that there is an achievement gap } \\
\text { between low-income students and affluent } \\
\text { students. Tell me, how do you make your } \\
\text { low-income students a spire to be } \\
\text { successful? }\end{array}$ & 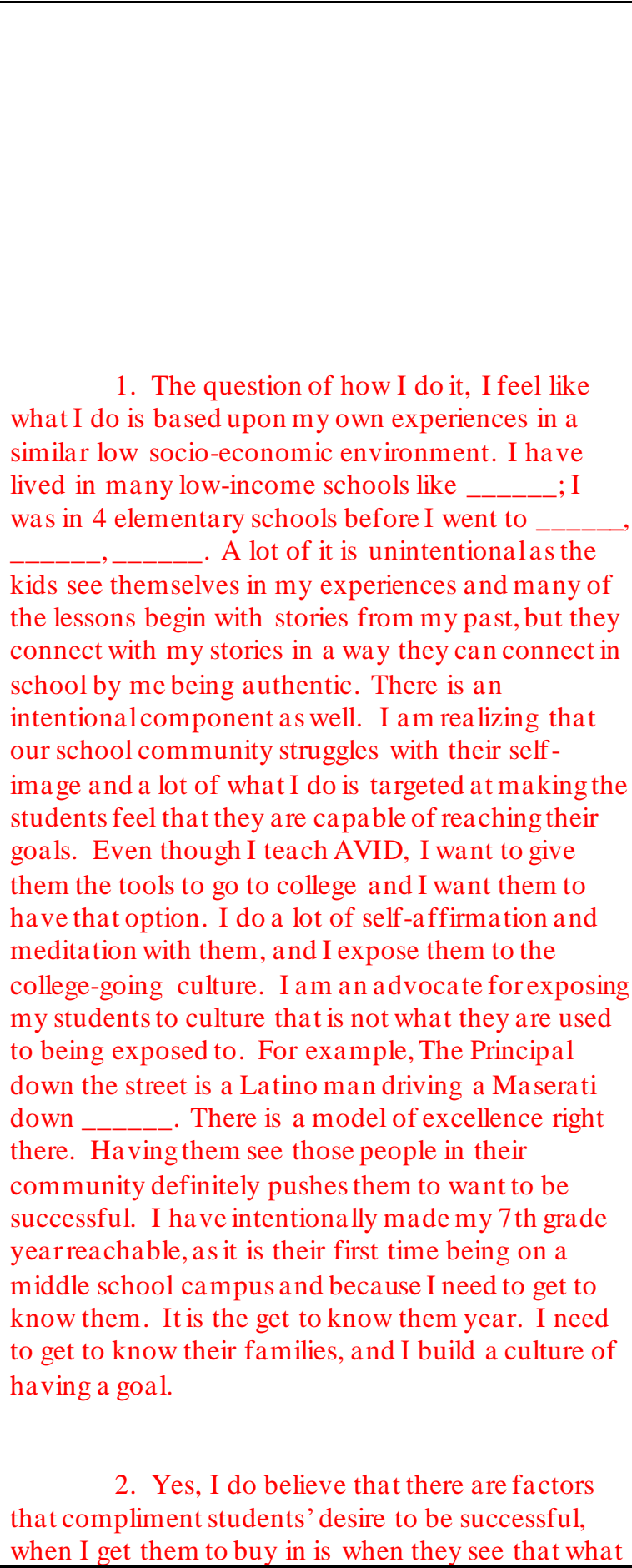 \\
\hline
\end{tabular}




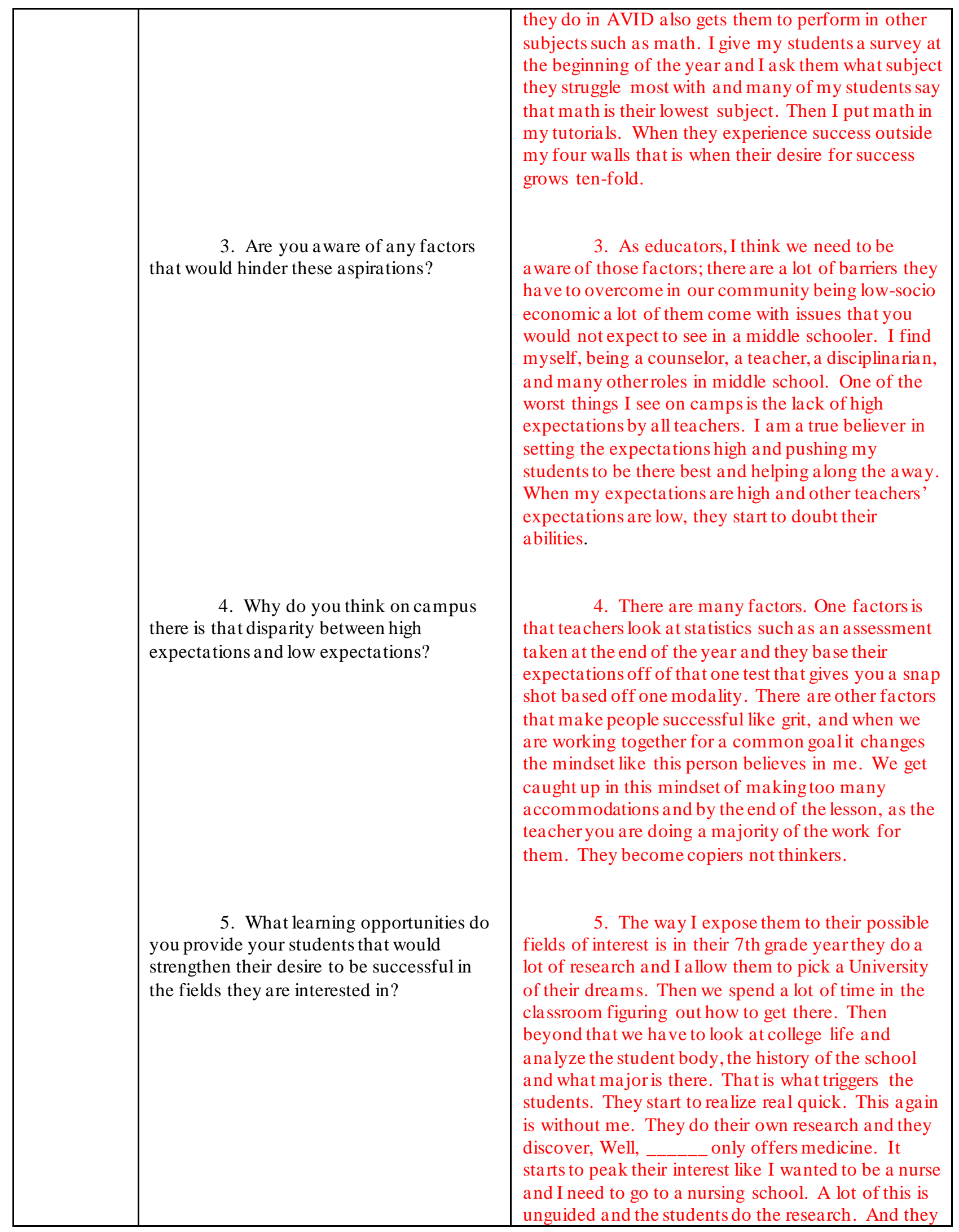




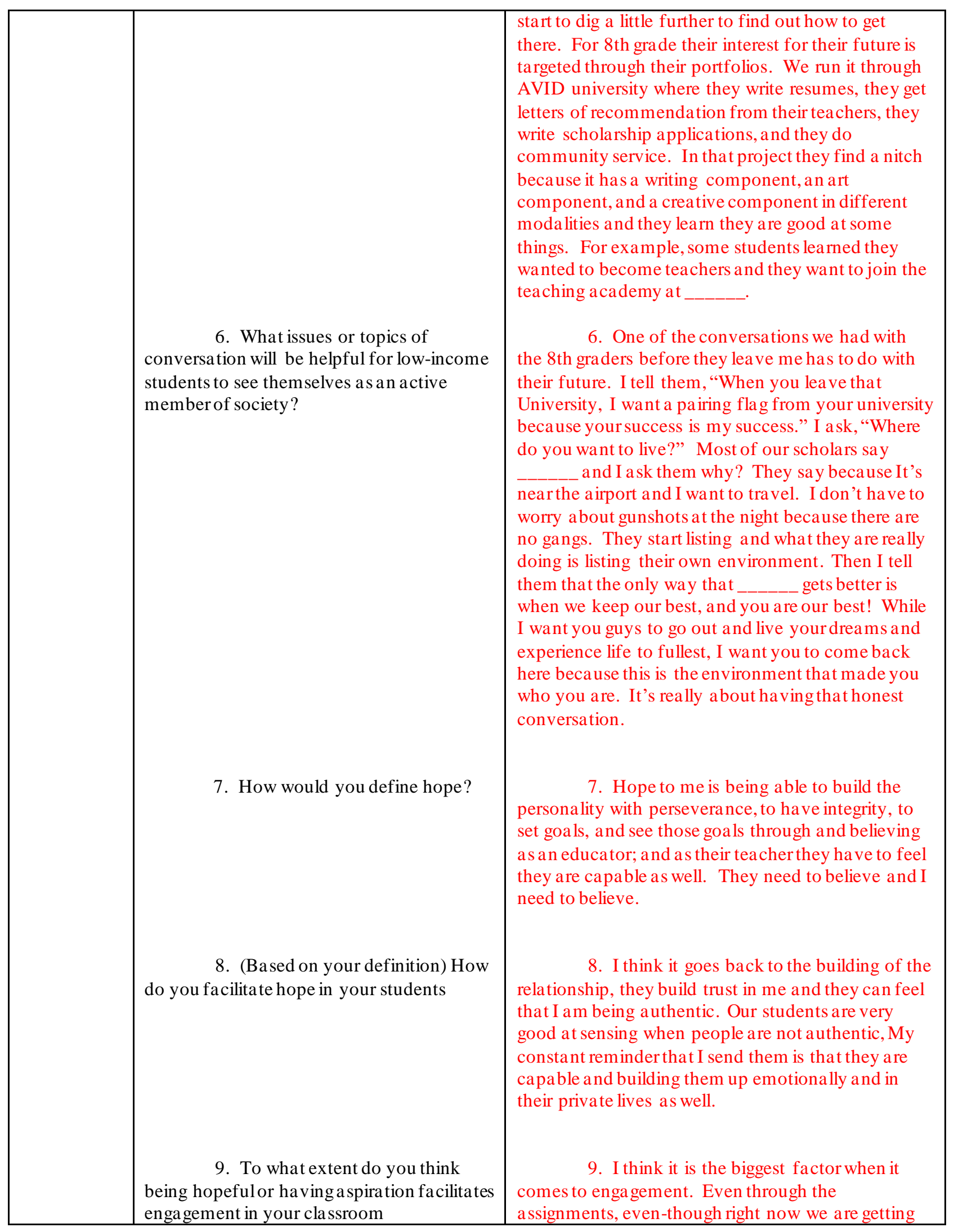




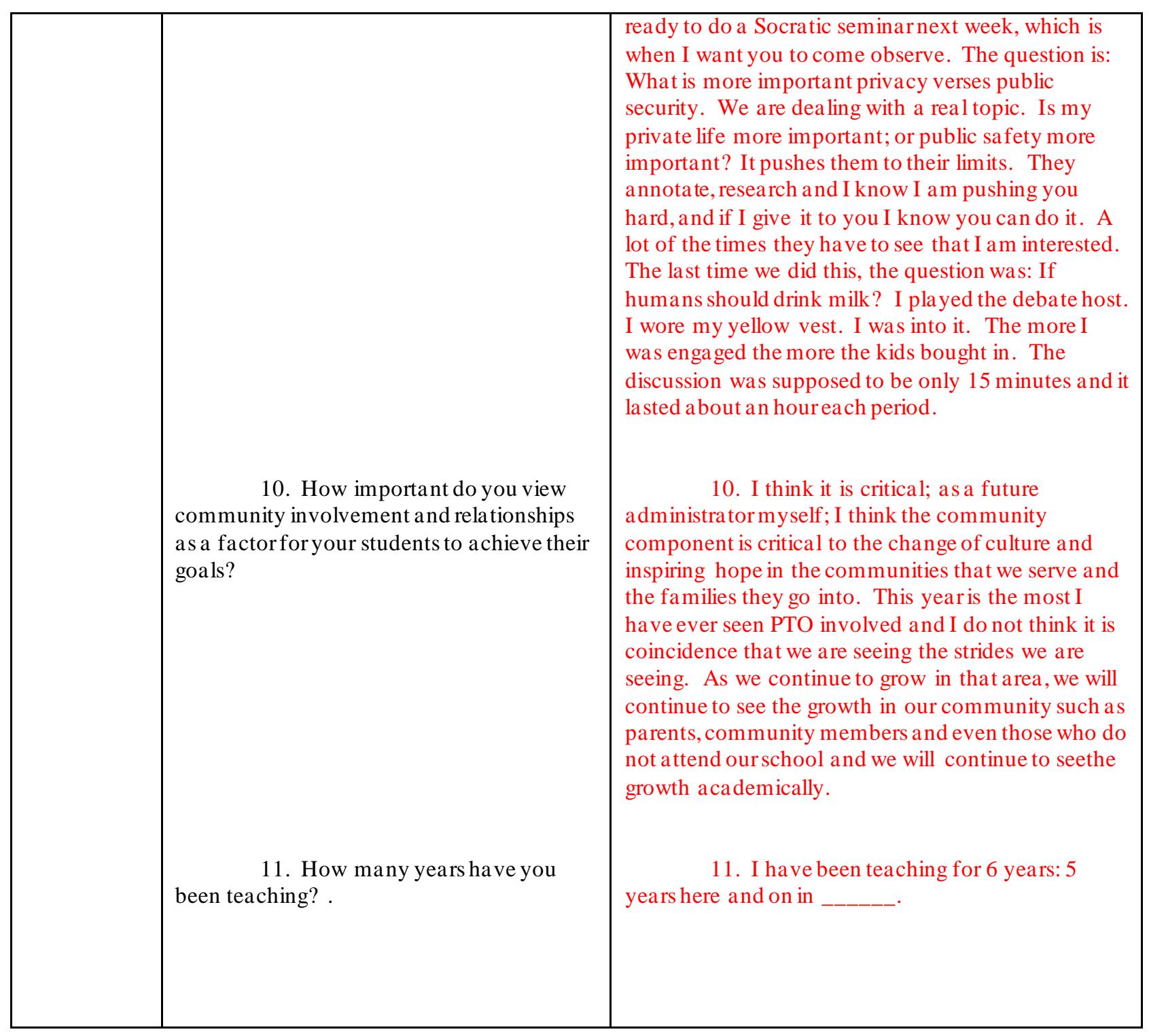


EXAMINING TEACHERS' PARADIGM AND PEDAGOGY IN MANIFESTING CRITICAL HOPE IN LOW-INCOME MIDDLE SCHOOL

Manifestations of Critical Hope Reflected in Teachers' Paradigm and Pedagogy

\section{Observation Matrix}

(For observing elements of Critical Hope manifested in teachers' language and behavior of hope, teachers' instructional delivery of hope, and teachers' lesson planning of hope)

\section{The Teacher \\ Time Observed: _ P-1}

Observer: _ D2

Date: 10:48 5/31/18

\begin{tabular}{|c|c|c|c|c|}
\hline $\begin{array}{l}\text { Manifestations of } \\
\text { Critical Hope } \\
\text { Elements in } \\
\text { Teachers' } \\
\text { Paradigm \& } \\
\text { Pedagogy } \\
\end{array}$ & $\begin{array}{l}\text { Manifestations } \\
\text { of Critical Hope } \\
\text { in: }\end{array}$ & $\begin{array}{l}\text { General } \\
\text { Indicators }\end{array}$ & Examples & Observed Evidence \\
\hline $\begin{array}{ll}\checkmark & \text { Goal Directed } \\
& \text { Thinking } \\
\checkmark & \text { Navigating } \\
& \text { Pathways } \\
\checkmark & \text { Willpower or } \\
& \text { Agency } \\
& \text { (Edwards, } \\
& \text { Rand,Lopez, \& } \\
& \text { Snyder, 2007) }\end{array}$ & $\begin{array}{l}\text { Teachers use } \\
\text { of vocabulary } \\
\text { expression and } \\
\text { written } \\
\text { direction }\end{array}$ & $\begin{array}{l}\text { - Language } \\
\text { and behavior } \\
\text { that connect, } \\
\text { connote, } \\
\text { imply } \\
\text { valuing and } \\
\text { trusting } \\
\text { optimistic } \\
\text { aspirations }\end{array}$ & $\begin{array}{ll}\circ & \begin{array}{l}\text { Positive } \\
\text { reinforce- } \\
\text { ments }\end{array} \\
\circ & \begin{array}{l}\text { Positive } \\
\text { directives }\end{array} \\
\circ & \text { Praises } \\
\circ & \text { Encouraging } \\
& \text { statements } \\
\circ & \text { Competence }\end{array}$ & $\begin{array}{l}\text { Students are sitting in } \\
\text { a large circle. There is calming } \\
\text { meditation music on in the } \\
\text { background. "Remember levels } \\
\text { of questioning. Level } 1 \text { is just } \\
\text { for definition. We don't need a } \\
\text { definition. Everyone in here } \\
\text { has done the work. We need } \\
\text { thoughtfulquestions. Raise } \\
\text { your hand if you need more } \\
\text { time. I'll give you one more } \\
\text { minute." Students are quietly } \\
\text { preparing for their Socratic } \\
\text { Seminar. This is an } 8 \text { th grade } \\
\text { AVID classroom. All the tables } \\
\text { have been pushed out of the } \\
\text { way, to make room for this } \\
\text { large circle. Teacherbegins } \\
\text { with addressing the "Rules of } \\
\text { Engagement." "I am just a } \\
\text { facilitatorin this setting. You } \\
\text { are not talking to me. You are } \\
\text { talking to the group. \#1 Be } \\
\text { prepared. I know you are } \\
\text { prepared. I see you with your } \\
\text { documents. Thank you for that. } \\
\text { \#2 Show Respect. This is not a } \\
\text { debate. This is just a natural }\end{array}$ \\
\hline
\end{tabular}




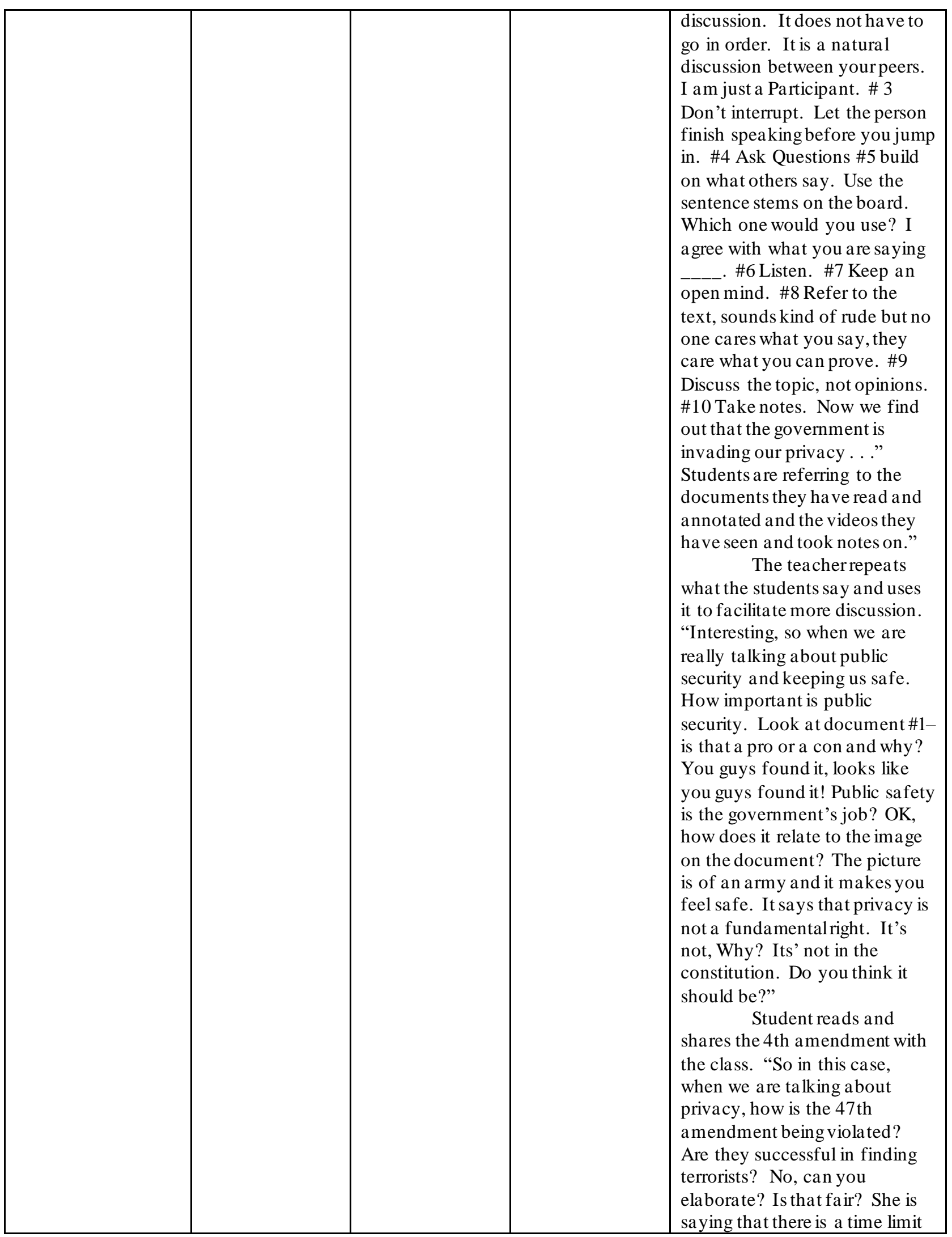




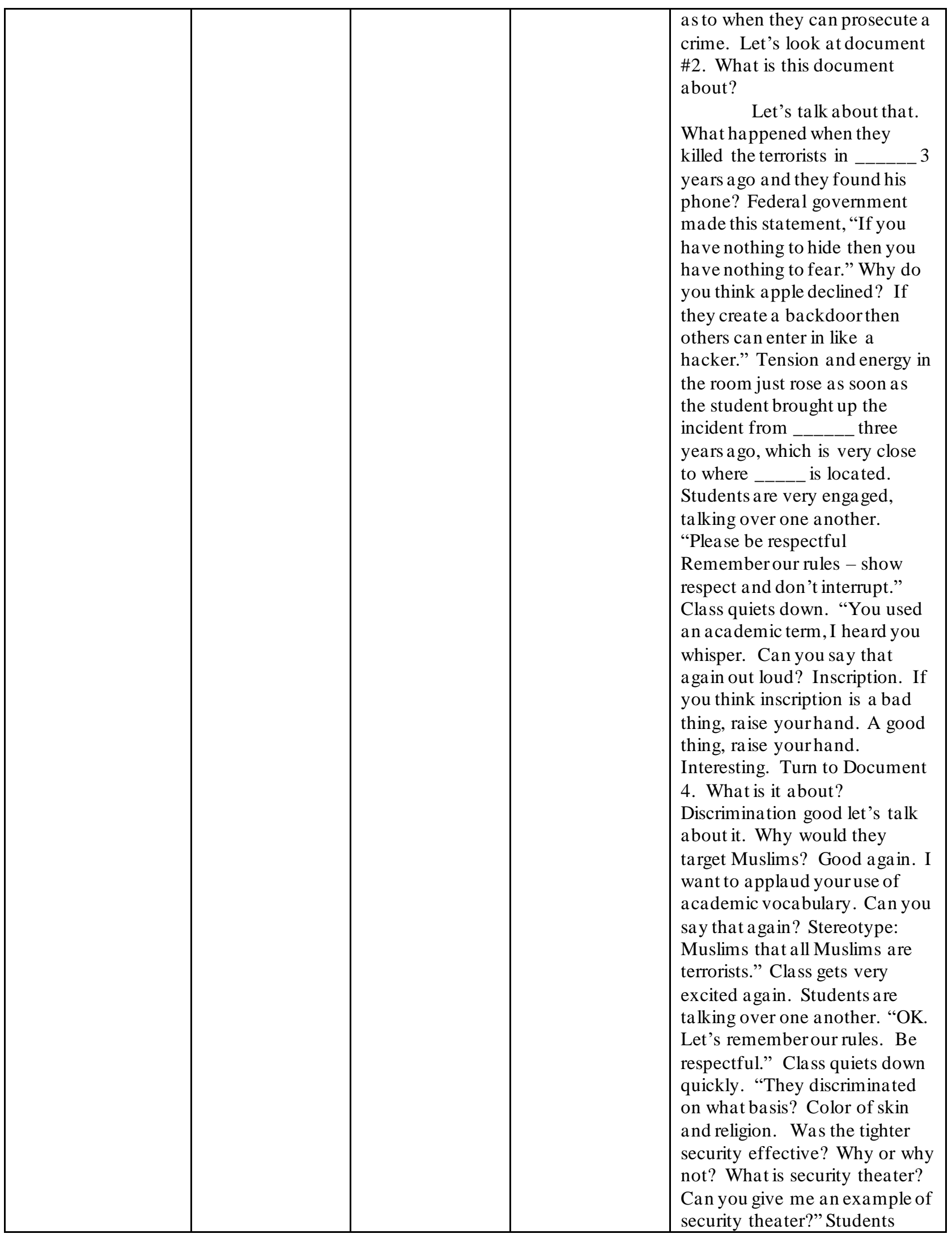




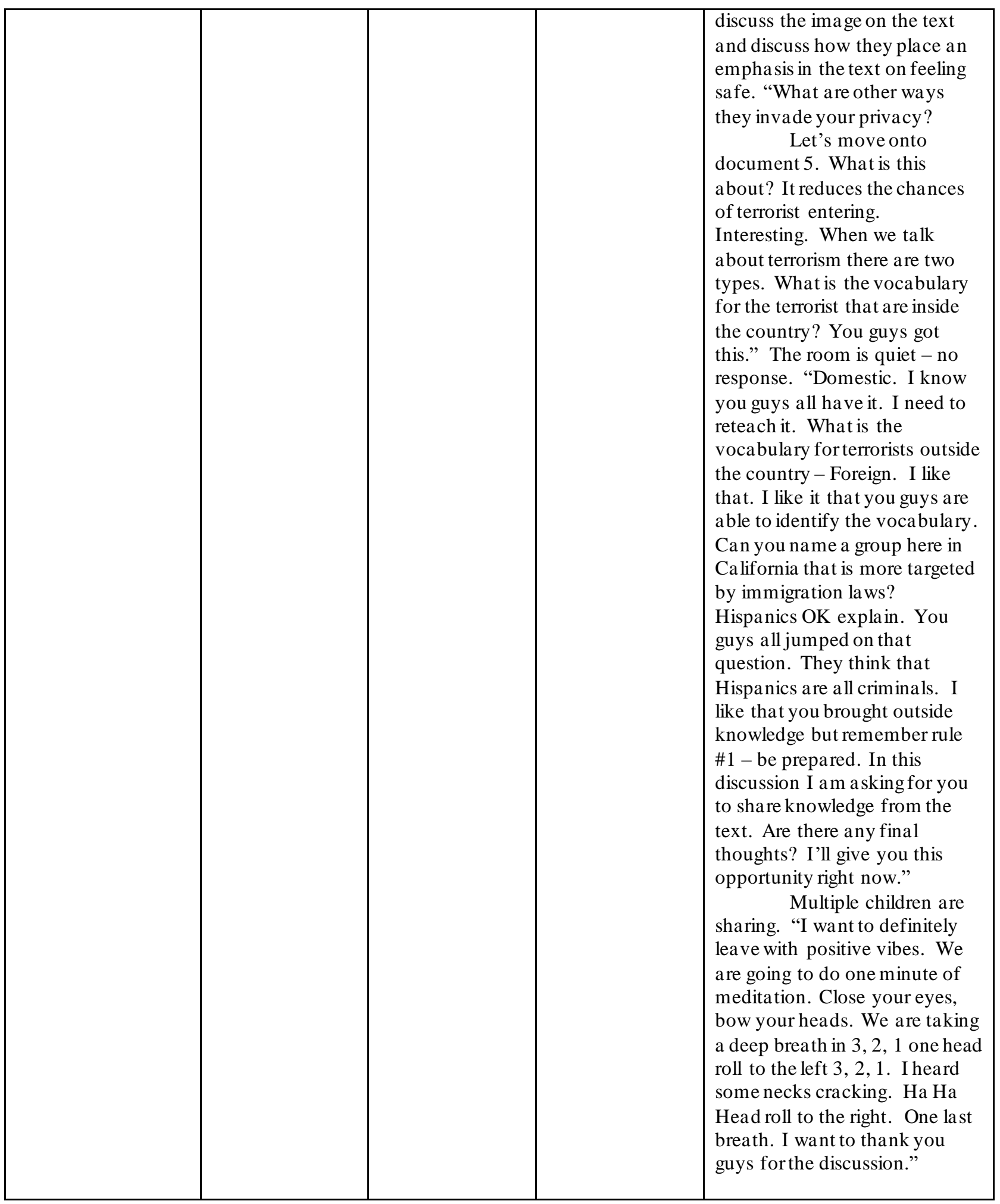




\begin{tabular}{|c|c|c|c|c|c|}
\hline $\begin{array}{l}\checkmark \\
\checkmark \\
\checkmark \\
\checkmark \\
\checkmark \\
\checkmark\end{array}$ & $\begin{array}{l}\text { Optimism } \\
\text { (Freire, 1997) } \\
\text { Ethics (Freire, } \\
\text { 1997) } \\
\text { Criticality } \\
\text { (Freire, 1974, } \\
\text { Giroux, 2011) } \\
\text { Social and } \\
\text { Historical } \\
\text { Consciousness } \\
\text { (Freire, 1974, } \\
\text { 1997) } \\
\text { Humanizing } \\
\text { Practices } \\
\text { (Freire, 1970, } \\
\text { 1974, 1994, } \\
\text { 1997) }\end{array}$ & $\begin{array}{l}\text { Teachers } \\
\text { prepared } \\
\text { written tasks } \\
\text { and } \\
\text { assignments }\end{array}$ & $\begin{array}{l}\text { Teacher } \\
\text { delivery in } \\
\text { prompting } \\
\text { critical } \\
\text { thinking and } \\
\text { aspirations } \\
\text { to be caring } \\
\text { socially } \\
\text { conscious } \\
\text { and ethical. }\end{array}$ & $\begin{array}{ll}\circ & \begin{array}{l}\text { Higher } \\
\text { levels of } \\
\text { questioning } \\
\text { Making } \\
\text { value }\end{array} \\
\text { judgments } \\
\text { that show } \\
\text { caring and } \\
\text { ethical } \\
\text { disposition } \\
\text { based upon } \\
\text { the criteria }\end{array}$ & \\
\hline $\begin{array}{l}\bar{\checkmark} \\
\checkmark\end{array}$ & $\begin{array}{l}\text { Sense of } \\
\text { Community } \\
\text { (hooks, 2003, } \\
\text { Freire, 1997) } \\
\text { Caring } \\
\text { Relationships } \\
\text { (Noddings, } \\
\text { 2017, Freire } \\
\text { 1997) } \\
\text { Knowledge of } \\
\text { Social \& } \\
\text { Cultural } \\
\text { Capital } \\
\text { (Bourdieu, } \\
\text { 1986) } \\
\text { Critical } \\
\text { Citizenry } \\
\text { (Giroux, 2011, } \\
\text { Freire, 1997) } \\
\text { Democratic } \\
\text { Advocacy } \\
\text { (Giroux, 2011) }\end{array}$ & $\begin{array}{l}\text { Teachers } \\
\text { composed } \\
\text { lesson plans }\end{array}$ & $\begin{array}{l}\text { Teacher } \\
\text { planning that } \\
\text { may elicit a } \\
\text { sense of } \\
\text { community } \\
\text { and } \\
\text { humanizing } \\
\text { practices. }\end{array}$ & $\begin{array}{l}\text { Lesson plans } \\
\text { that requires } \\
\text { collaboration } \\
\text { and } \\
\text { creativity in } \\
\text { manifesting } \\
\text { voice and } \\
\text { advocacy } \\
\text { that reflect } \\
\text { sense of } \\
\text { community } \\
\text { and } \\
\text { humanizing } \\
\text { practices }\end{array}$ & \\
\hline & & \multicolumn{4}{|c|}{$\begin{array}{l}\text { Other observations: This classroom is highly rigorous. Students have had to } \\
\text { ad six documents and annotate, and create leveled questions to be prepared for this } \\
\text { scussion. There is nothing fancy in this room expect for the high level of expectations } \\
\text { id pedagogical instruction. Big smiles on the teacher's face. He enjoys his job. There } \\
\text { e posters on the wall about AVID that students have created and hand-written } \\
\text { ntence stems on the white board. Conversations are deep and rich and students are } \\
\text { ghly engaged. Everyone was on task, listening, contributing, and supporting one } \\
\text { lother. There are currently, zero behavioral issues in this room. }\end{array}$} \\
\hline
\end{tabular}


EXAMINING TEACHERS' PARADIGM AND PEDAGOGY IN MANIFESTING CRITICAL
HOPE IN LOW-INCOME MIDDLE SCHOOL

Appendix B

Participant 2: Interview and Observation

\begin{tabular}{|c|c|c|}
\hline $\begin{array}{l}\text { Participant } \\
\text { P.2 5/24/19 }\end{array}$ & Questions & Comments \\
\hline \multirow[t]{2}{*}{ Teacher } & $\begin{array}{l}\text { Introduction: Thank you for a greeing } \\
\text { to participate in my study on Examining } \\
\text { Teachers' Paradigm and Pedagogy in } \\
\text { Manifesting Critical Hope in Low-Income } \\
\text { Middle School Cla ssrooms: A Narrative } \\
\text { Inquiry } \\
\text { This interview will last approximately } \\
30 \text { to } 45 \text { minutes. I will be a sking you to } \\
\text { review the transcript of the interview for } \\
\text { accuracy. } \\
\text { 1. (PI's introduction to questions) } \\
\text { START WITH an OPEN-ENDED } \\
\text { QUESTION: JUST LIKE TELLING A } \\
\text { STORY: We already know through existing } \\
\text { research that there is an achievement gap } \\
\text { between low-income students and affluent } \\
\text { students. Tell me, how do you make your low- } \\
\text { income students a spire to be successful? } \\
\text { Follow-up question: Can you provide } \\
\text { an example? }\end{array}$ & 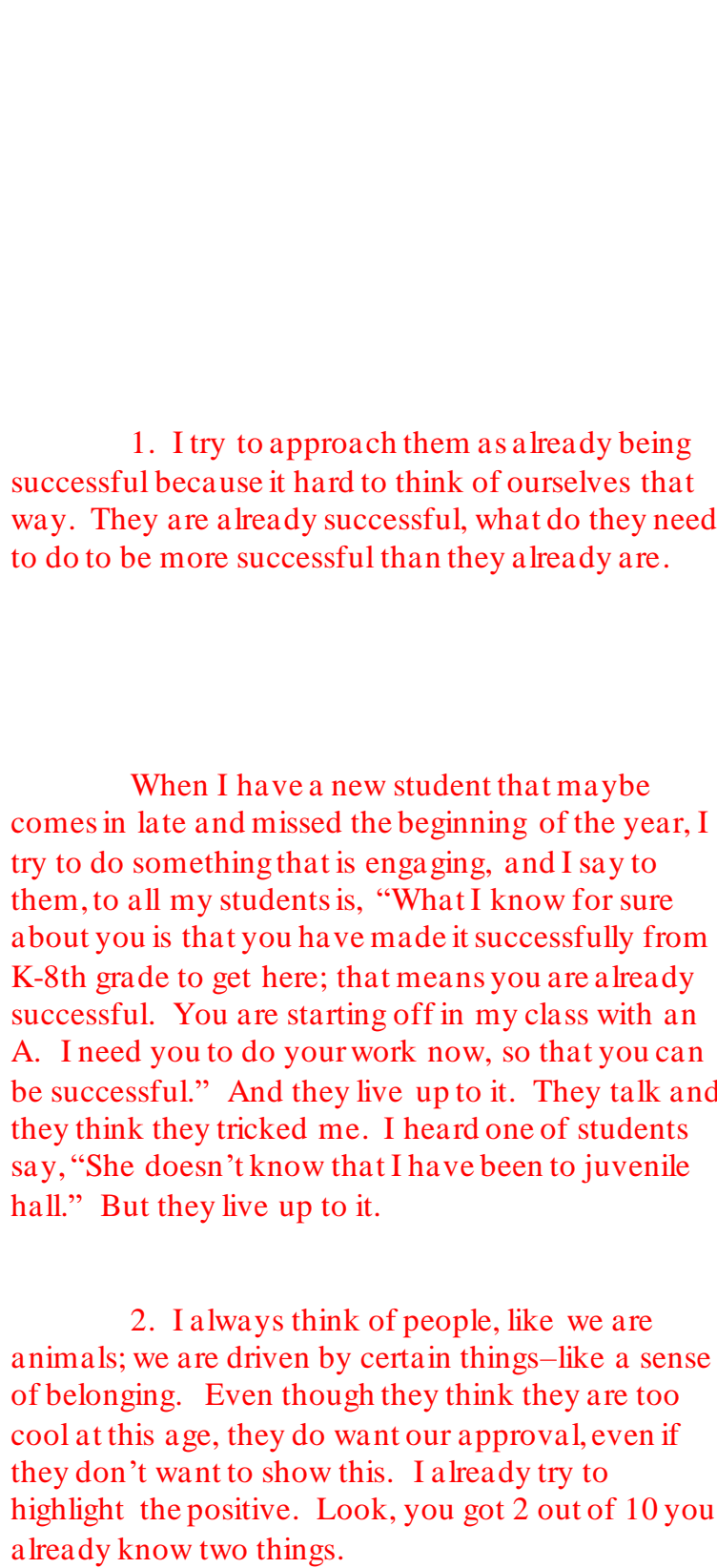 \\
\hline & $\begin{array}{l}\text { 3. Are you aware of any factors that } \\
\text { would hinder these aspirations? }\end{array}$ & $\begin{array}{l}\text { 3. Any difficulties that they experience in their } \\
\text { personal lives and I think that finding a middle } \\
\text { ground in the classroom to where they are being held }\end{array}$ \\
\hline
\end{tabular}




\section{EXAMINING TEACHERS' PARADIGM AND PEDAGOGY IN MANIFESTING CRITICAL HOPE IN LOW-INCOME MIDDLE SCHOOL}

4. Why do you think on campus there is that disparity between high expectations and low expectations?

5. What learning opportunities do you provide your students that would strengthen their desire to be successful in the fields they are interested in?

6. What issues or topics of conversation will be helpful for low-income students to see themselves as an active member of society?

7. How would you define hope?

8. (Based on your definition) How do you facilitate hope in your students to expectations, there is punishment, and at the same time there is, a new start, we start over tomorrow. If they feel they are the ones that are bad, instead their behavior that is a poor choice. Then that will hinder their aspirations.

4. I think we confuse our kids with their behavior sometimes. Behaviors can be very outra geous and personally offensive, but at the end of the day, they are an expression of a need that isn't being met. If you can connect it to that, you can move past the behavior and into the person; and if you can't, that person is that behavior and they are then a problem.

5. During our career week, we have a career research project and everyone researches the career they are interested in. The goal is to figure it out. How much money will I make? How much education will I need? I have seen kids who already know how to fill out that form because they have already done the research and I see others who fill out that form and discover that they don't want to be for example, a photographer because they will not make that much money and they realize they are one step closer to what they want to do by discovering out this isn't for me.

6. I think always seeing others like themselves who are in positions of opportunity will always be something that is helpful for them, which is why I always wanted to teach in a community, which is like mine, in is very

similar to the student population in I read about something called a stereotype threat, which is not other people stereotyping, but its themselves, how they see themselves, that is limiting them from reaching their potential. Always talking about them and telling them that you are capable and you are able just as much as anyone else. I think those things are helpful.

7. I would define hope as the coura ge to dream and to pursue those dreams.

8. With goal setting, with a career-day project, I try to guide them towards always having something specific they are working towards. So that they understand there is a cycle: set a goal, try, 


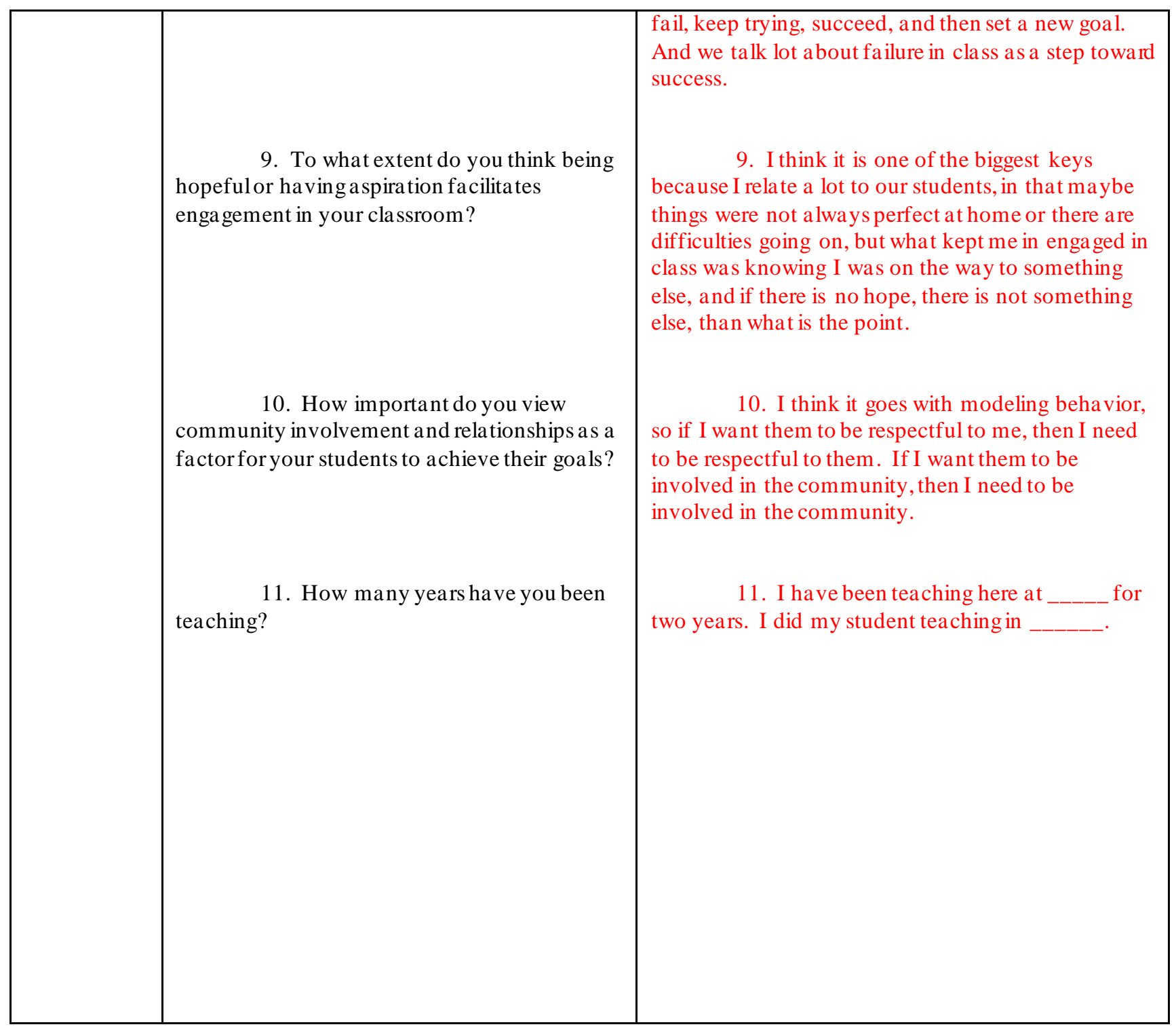


EXAMINING TEACHERS' PARADIGM AND PEDAGOGY IN MANIFESTING CRITICAL HOPE IN LOW-INCOME MIDDLE SCHOOL

\section{Manifestations of Critical Hope Reflected in Teachers' Paradigm and Pedagogy}

\section{Observation Matrix}

(For observing elements of Critical Hope manifested in teachers' language and behavior of hope, teachers' instructional delivery of hope, and teachers' lesson planning of hope)

\section{The Teacher}

Time Observed: __P-2
Observer:
F5
Date: 1:28 5/23/18

\begin{tabular}{|c|c|c|c|c|}
\hline $\begin{array}{l}\text { Manifestations of } \\
\text { Critical Hope } \\
\text { Elements in } \\
\text { Teachers' Paradigm } \\
\text { \& Pedagogy }\end{array}$ & $\begin{array}{l}\text { Manifestation } \\
\text { s of Critical } \\
\text { Hope in: }\end{array}$ & $\begin{array}{l}\text { General } \\
\text { Indicators }\end{array}$ & Examples & Observed Evidence \\
\hline $\begin{aligned} \checkmark & \text { Goal Directed } \\
& \text { Thinking } \\
\checkmark & \text { Navigating } \\
& \text { Pathways } \\
\checkmark & \text { Criticality } \\
& \text { (Freire, 1974, } \\
& \text { Giroux, 2011) } \\
\checkmark & \text { Social } \\
& \text { Willpower or } \\
& \text { Agency } \\
& \text { (Edwards, } \\
& \text { Rand,Lopez, \& } \\
& \text { Snyder, 2007) }\end{aligned}$ & $\begin{array}{l}\text { Teachers use } \\
\text { of vocabulary } \\
\text { expression } \\
\text { and written } \\
\text { direction }\end{array}$ & $\begin{array}{l}\text { - Language } \\
\text { and behavior } \\
\text { that connect, } \\
\text { connote, } \\
\text { imply } \\
\text { valuing and } \\
\text { trusting } \\
\text { optimistic } \\
\text { a spirations }\end{array}$ & $\begin{array}{ll}\circ & \begin{array}{l}\text { Positive } \\
\text { reinforcements }\end{array} \\
\circ & \begin{array}{l}\text { Positive } \\
\text { directives }\end{array} \\
\circ & \text { Praises } \\
\circ & \text { Encouraging } \\
\text { statements } \\
\circ & \text { Competence }\end{array}$ & $\begin{array}{l}\text {-Good! It actually } \\
\text { improved the time! So what } \\
\text { is your next move? } \\
\text {-Nice! What else can we } \\
\text { do? } \\
\text {-Wow! That works great! }\end{array}$ \\
\hline $\begin{array}{ll}\checkmark & \text { Optimism } \\
& \text { (Freire, 1997) } \\
\checkmark & \text { Ethics (Freire, } \\
& \text { 1997) } \\
\checkmark & \text { and Historical } \\
& \text { Consciousness } \\
& \text { (Freire, 1974, } \\
& \text { 1997) } \\
\checkmark & \text { Humanizing } \\
& \text { Practices } \\
& \text { (Freire, 1970, } \\
1974,1994, \\
1997)\end{array}$ & $\begin{array}{l}\text { Teachers } \\
\text { prepared } \\
\text { written tasks } \\
\text { and } \\
\text { assignments }\end{array}$ & $\begin{array}{l}\text { Teacher } \\
\text { delivery in } \\
\text { prompting } \\
\text { critical } \\
\text { thinking and } \\
\text { a spirations to } \\
\text { be caring } \\
\text { socially } \\
\text { conscious } \\
\text { and ethical. }\end{array}$ & 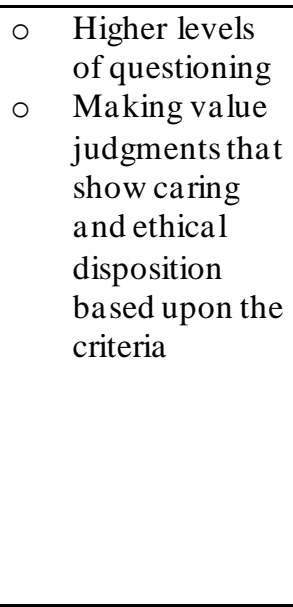 & $\begin{array}{l}\text {-Gentleman, you are in } \\
\text { this group. I need everyone } \\
\text { working together on the } \\
\text { project to solve it ok? } \\
\text {-Teacher is explaining } \\
\text { the incline: You see how it } \\
\text { looks straight, but there is } \\
\text { enough of an incline to create } \\
\text { motion. It doesn't have to } \\
\text { have such a big drop. } \\
\text { Here is what I would } \\
\text { suggest: Someone in the } \\
\text { group make a sharp turn and } \\
\text { someone in the group make a } \\
\text { slight turn and see what }\end{array}$ \\
\hline
\end{tabular}




\begin{tabular}{|c|c|c|c|c|c|}
\hline & & & & & works best. \\
\hline \multicolumn{2}{|c|}{ 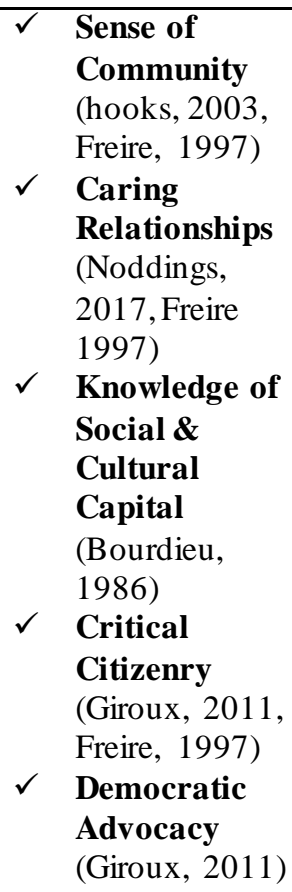 } & $\begin{array}{l}\text { Teachers } \\
\text { composed } \\
\text { lesson plans }\end{array}$ & $\begin{array}{l}\text { - Teacher } \\
\text { planning that } \\
\text { may elicit a } \\
\text { sense of } \\
\text { community } \\
\text { and } \\
\text { humanizing } \\
\text { practices. }\end{array}$ & $\begin{array}{l}\text { Lesson plans } \\
\text { that requires } \\
\text { collaboration } \\
\text { and creativity } \\
\text { in manifesting } \\
\text { voice and } \\
\text { advocacy that } \\
\text { reflect sense of } \\
\text { community } \\
\text { and } \\
\text { humanizing } \\
\text { practices }\end{array}$ & $\begin{array}{l}\text { This project is a } \\
\text { collaborative group project } \\
\text { on building a roller coaster } \\
\text { out of paper and tape and } \\
\text { working together to problem } \\
\text { solve to expand the time it } \\
\text { takes for the marble to go } \\
\text { from the top to the bottom. }\end{array}$ \\
\hline & & \multicolumn{4}{|c|}{$\begin{array}{l}\text { Other observations: The room is exciting. Jazz music playing in the } \\
\text { background. Students are working collaboratively in table groups making roller } \\
\text { coasters out of paper and tape. They are standing about } 4 \text { feet high and are colorful } \\
\text { because of the different colors of paper. They are trying to improve their times. They } \\
\text { put a marble in on the top and time it on their cell phones how long it takes to get to } \\
\text { the bottom. The task is to increase the time to at least } 15 \text { seconds. Class is lively, } \\
\text { students are excited and happy, and the teacher is acting as a facilitator, going to each } \\
\text { group asking questions and explains the concepts to get them to continue to improve } \\
\text { their roller coasters. Students have named their roller coasters. One has a sign on it } \\
\text { that says "DOPE." Conversations are rich. }\end{array}$} \\
\hline
\end{tabular}




\section{EXAMINING TEACHERS' PARADIGM AND PEDAGOGY IN MANIFESTING CRITICAL HOPE IN LOW-INCOME MIDDLE SCHOOL}

Appendix C

Participant 3: Interview and Observation

\begin{tabular}{|c|c|c|}
\hline $\begin{array}{l}\text { Participant } \\
\text { P.3 5/29/19 }\end{array}$ & Questions & Comments \\
\hline Teacher & $\begin{array}{l}\text { Introduction: Thank you for } \\
\text { agreeing to participate in my study on } \\
\text { Examining Teachers' Paradigm and Pedagogy } \\
\text { in Manifesting Critical Hope in Low-Income } \\
\text { Middle School Classrooms: A Narrative } \\
\text { Inquiry } \\
\text { This interview will last } \\
\text { approximately } 30 \text { to } 45 \text { minutes. I will be } \\
\text { a sking you to review the transcript of the } \\
\text { interview for accuracy. } \\
\text { 1. (PI's introduction to questions) } \\
\text { START WITH an OPEN-ENDED } \\
\text { QUESTION: JUST LIKE TELLING A } \\
\text { STORY: We a lready know through existing } \\
\text { research that there is an achievement gap } \\
\text { between low-income students and affluent } \\
\text { students. Tell me, how do you make your } \\
\text { low-income students a spire to be successful? } \\
\text { F. Are you aware of any factors that } \\
\text { Follow-up question: Can you provide } \\
\text { an example? }\end{array}$ & $\begin{array}{l}\text { 1. I like to share my own experience because } \\
\text { I was also a low-income student and I am from this } \\
\text { community and I tell them that I have been there } \\
\text { where you are and if I can do it you can do it too. } \\
\text { You need a support system. My family was my } \\
\text { support system. I want to be their support system. I } \\
\text { tell them even when you go to high school come back } \\
\text { and I will be here for you. In class, we use what we } \\
\text { have. } \\
\qquad \text { We make it work. When I run out of graph } \\
\text { paper we adjust. When my projector does not work } \\
\text { we make it happen. Just little things like that. } \\
\text { 2. Acknowledging them and making them } \\
\text { feel valued in the classroom and even out of the } \\
\text { classroom like going to their games and stuff. Letting } \\
\text { them know they have my support. When they } \\
\text { apologize, I let them know that I will always be there } \\
\text { for them and by consistently supporting them through } \\
\text { the good and the bad. }\end{array}$ \\
\hline
\end{tabular}




\section{EXAMINING TEACHERS' PARADIGM AND PEDAGOGY IN MANIFESTING CRITICAL HOPE IN LOW-INCOME MIDDLE SCHOOL}

\begin{tabular}{|c|c|}
\hline $\begin{array}{l}\text { 4. Why do you think on campus } \\
\text { there is that disparity between high } \\
\text { expectations and low expectations? }\end{array}$ & $\begin{array}{l}\text { 4. It has a lot to do with the teachers and } \\
\text { what they believe their students can do. Like I said, I } \\
\text { came from nothing, so it's possible but I don't think } \\
\text { all teachers understand this. I think high expectations } \\
\text { come with care, with building a relationship, the } \\
\text { deeper your relationship; the more the students will } \\
\text { reach these high expectations. The more you care the } \\
\text { more they will reach. }\end{array}$ \\
\hline $\begin{array}{l}\text { 5. What learning opportunities do } \\
\text { you provide your students that would } \\
\text { strengthen their desire to be successful in the } \\
\text { fields they are interested in? }\end{array}$ & $\begin{array}{l}\text { 5. I try to relate the math to real life, so they } \\
\text { can apply what they know and what they are } \\
\text { interested in. I try to push them to be the best they } \\
\text { can be. I tell them, I know you can do this, you just } \\
\text { got to focus. I know they can do this. }\end{array}$ \\
\hline $\begin{array}{l}\text { 6. What issues or topics of } \\
\text { conversation will be helpful for low-income } \\
\text { students to see themselves as an active } \\
\text { member of society? }\end{array}$ & $\begin{array}{l}\text { 6. You can't really read it from a book and } \\
\text { when they do its general that's not my life. When } \\
\text { they see someone from their community then it's } \\
\text { more valuable. I want to be that for you guys. I want } \\
\text { to be that for you. I want you to start thinking about } \\
\text { your future now. }\end{array}$ \\
\hline 7. How would you define hope? & $\begin{array}{l}\text { 7. Hope, I would say is like. I think hope is } \\
\text { like having a positive mindset toward a situation. } \\
\text { Toward something you want or need. }\end{array}$ \\
\hline $\begin{array}{l}\text { 8. (Based on your definition) How } \\
\text { do you facilitate hope in your students }\end{array}$ & $\begin{array}{l}\text { 8. I would be consistently reinforcing that I } \\
\text { believe in them. I try to build a classroom where it is } \\
\text { safe, so they can feel comfortable enough to let down } \\
\text { their guard, so they can achieve. I support their } \\
\text { academic needs; their interests, their goals, and I try to } \\
\text { have them focus on having a positive mindset. }\end{array}$ \\
\hline $\begin{array}{l}\text { 9. To what extent do you think being } \\
\text { hopefulor having a spiration facilitates } \\
\text { engagement in your classroom? }\end{array}$ & $\begin{array}{l}\text { 9. I think that is a big part of the classroom } \\
\text { because the students have to know that you care and } \\
\text { believe in them. I try to have that come first; to build } \\
\text { a relationship with them. Coming from the same } \\
\text { community I feel our students need this. I needed one } \\
\text { teacher to really see me not just look at a class of } 35 \text {, } \\
\text { but really see me. I try to see them, to see their goals } \\
\text { and their dreams, and I try to support them in reaching } \\
\text { them. }\end{array}$ \\
\hline $\begin{array}{l}\text { 10. How important do you view } \\
\text { community involvement and rela tionships as a } \\
\text { factor for your students to achieve their goals? }\end{array}$ & $\begin{array}{l}10 \text {. I think it is really important because the } \\
\text { students have to see you out participating in after- } \\
\text { school things in the community because that shows } \\
\text { you care that you took the time to come see them after } \\
\text { school, to go to their a wards assembly, and to build }\end{array}$ \\
\hline
\end{tabular}


EXAMINING TEACHERS' PARADIGM AND PEDAGOGY IN MANIFESTING CRITICAL HOPE IN LOW-INCOME MIDDLE SCHOOL

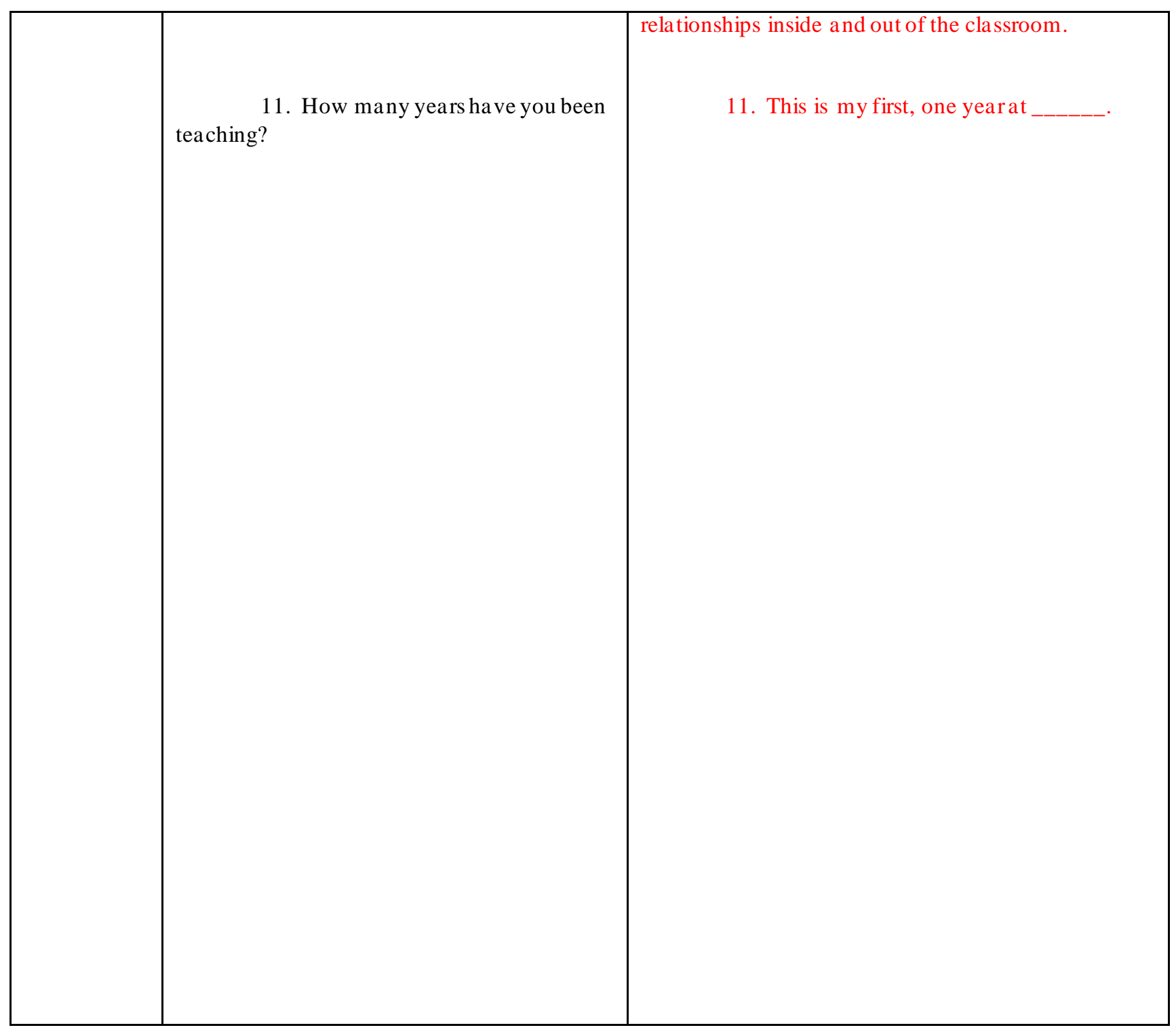


EXAMINING TEACHERS' PARADIGM AND PEDAGOGY IN MANIFESTING CRITICAL HOPE IN LOW-INCOME MIDDLE SCHOOL

\section{Manifestations of Critical Hope Reflected in Teachers' Paradigm and Pedagogy}

\section{Observation Matrix}

(For observing elements of Critical Hope manifested in teachers' language and behavior of hope, teachers' instructional delivery of hope, and teachers' lesson planning of hope)

\section{The Teacher}

Time Observed: __ P-3
Observer: $\quad \mathbf{E} 4$
Date: 1:36 5/23/18

\begin{tabular}{|c|c|c|c|c|}
\hline $\begin{array}{l}\text { Manifestations of } \\
\text { Critical Hope } \\
\text { Elements in } \\
\text { Teachers' } \\
\text { Paradigm \& } \\
\text { Pedagogy }\end{array}$ & $\begin{array}{l}\text { Manifestations } \\
\text { of Critical } \\
\text { Hope in: }\end{array}$ & $\begin{array}{l}\text { General } \\
\text { Indicators }\end{array}$ & Examples & Observed Evidence \\
\hline $\begin{array}{ll}\checkmark & \text { Goal } \\
& \text { Directed } \\
& \text { Thinking } \\
\checkmark & \text { Navigating } \\
& \text { Pathways } \\
\checkmark & \text { Willpower or } \\
& \text { Agency } \\
\text { (Edwards, } \\
\text { Rand,Lopez, } \\
\text { \& Snyder, } \\
\text { 2007) }\end{array}$ & $\begin{array}{l}\text { Teachers use } \\
\text { of vocabulary } \\
\text { expression } \\
\text { and written } \\
\text { direction }\end{array}$ & $\begin{array}{l}\text { - Language } \\
\text { and } \\
\text { behavior } \\
\text { that } \\
\text { connect, } \\
\text { connote, } \\
\text { imply } \\
\text { valuing and } \\
\text { trusting } \\
\text { optimistic } \\
\text { a spirations }\end{array}$ & $\begin{array}{cl}\circ & \begin{array}{l}\text { Positive } \\
\text { reinforcements }\end{array} \\
\circ & \text { Positive } \\
\text { directives } \\
\circ & \text { Praises } \\
\circ & \text { Encouraging } \\
& \text { statements } \\
\circ & \text { Competence }\end{array}$ & $\begin{array}{l}\text {-Ok. Right now we } \\
\text { need to be writing } \\
\text {-Whether you are done } \\
\text { or not, you need to hit submit so } \\
\text { you can get points for this } \\
\text { week. } \\
\text {-I am going to wait } \\
\text { until we have a voice level of } 0 . \\
\quad \text { You will need to listen } \\
\text { or you will get lost. } \\
\text { (Walking through } \\
\text { explicit direct instructions) } \\
\text { Click on home, } 9 \text { th grade } \\
\text { project, so there are two parts. } \\
\quad \text { We are going to be } \\
\text { building a city. Before we do } \\
\text { this, we need to spend time on } \\
\text { vocabulary, academic } \\
\text { vocabulary that we have forgot. } \\
\text { O.K. I am going to explain, but } \\
\text { hold on. (Stops to dealwith } \\
\text { behavioralissues.) Here is how } \\
\text { I want you to set up your paper. } \\
\text { Write the words, definition, } \\
\text { Google it and cite where you } \\
\text { found it. And draw a picture. } \\
\text { You have } 18 \text { min. to complete } 9 \\
\text { words. I will set the timer for } 2\end{array}$ \\
\hline
\end{tabular}




\begin{tabular}{|c|c|c|c|c|c|c|}
\hline & & & & & & $\begin{array}{l}\text { minutes. You have } 2 \text { minutes } \\
\text { per word. Let's turn off our } \\
\text { voices. Now we need to finish } \\
\text { this today. Appropriate voice } \\
\text { level for this is } 0 \text {. You are } \\
\text { working on your own. You } \\
\text { need all components. }\end{array}$ \\
\hline $\begin{array}{l}\checkmark \\
\checkmark \\
\checkmark \\
\checkmark \\
\checkmark \\
\checkmark \\
\checkmark \\
\checkmark\end{array}$ & $\begin{array}{l}\text { Optimism } \\
\text { (Freire, } \\
\text { 1997) } \\
\text { Ethics } \\
\text { (Freire, } \\
\text { 1997) } \\
\text { Criticality } \\
\text { (Freire, } \\
\text { 1974, } \\
\text { Giroux, } \\
\text { 2011) } \\
\text { Social and } \\
\text { Historical } \\
\text { Consciousn } \\
\text { ess (Freire, } \\
\text { 1974, 1997) } \\
\text { Humanizing } \\
\text { Practices } \\
\text { (Freire, } \\
\text { 1970, 1974, } \\
\text { 1994, 1997) }\end{array}$ & $\begin{array}{l}\text { Teachers } \\
\text { prepared } \\
\text { written tasks } \\
\text { and } \\
\text { assignments }\end{array}$ & $\begin{array}{l}\text { Teacher } \\
\text { delivery in } \\
\text { prompting } \\
\text { critical } \\
\text { thinking } \\
\text { and } \\
\text { a spirations } \\
\text { to be caring } \\
\text { socially } \\
\text { conscious } \\
\text { and ethical. }\end{array}$ & & $\begin{array}{l}\text { Higher levels of } \\
\text { questioning } \\
\text { Making value } \\
\text { judgments that } \\
\text { show caring and } \\
\text { ethical } \\
\text { disposition based } \\
\text { upon the criteria }\end{array}$ & \\
\hline $\begin{array}{l}\checkmark \\
\checkmark \\
\checkmark \\
\checkmark \\
\checkmark \\
\checkmark \\
\end{array}$ & $\begin{array}{l}\text { Sense of } \\
\text { Community } \\
\text { (hooks, 2003, } \\
\text { Freire, 1997) } \\
\text { Caring } \\
\text { Relationships } \\
\text { (Noddings, } \\
\text { 2017, Freire } \\
\text { 1997) } \\
\text { Knowledge } \\
\text { of Social \& } \\
\text { Cultural } \\
\text { Capital } \\
\text { (Bourdieu, } \\
\text { 1986) } \\
\text { Critical } \\
\text { Citizenry } \\
\text { (Giroux, } \\
\text { 2011, Freire, } \\
\text { 1997) } \\
\text { Democratic } \\
\text { Advocacy } \\
\text { (Giroux, } \\
\text { 2011) }\end{array}$ & $\begin{array}{c}\text { Teachers } \\
\text { composed } \\
\text { lesson plans }\end{array}$ & $\begin{array}{l}\text { Teacher } \\
\text { planning } \\
\text { that may } \\
\text { elicit a } \\
\text { sense of } \\
\text { community } \\
\text { and } \\
\text { humanizing } \\
\text { practices. }\end{array}$ & & $\begin{array}{l}\text { Lesson plans } \\
\text { that requires } \\
\text { collaboration } \\
\text { and creativity in } \\
\text { manifesting } \\
\text { voice and } \\
\text { advocacy that } \\
\text { reflect sense of } \\
\text { community and } \\
\text { humanizing } \\
\text { practices }\end{array}$ & \\
\hline
\end{tabular}




\begin{tabular}{|l|l|}
\hline \multicolumn{1}{|c|}{ Other observations: Students are all siting in groups of 4 but they are all } \\
working individually in isolation. They are not allowed to speak or talk to their \\
neighbor. Voice level is 0. They all have ipads and are using them to work on this \\
vocabulary. Everyone has a lined sheet of paper and they are drawing four columns. \\
Class is quiet, there are students making abrupt noises. Teacher seems agitated and \\
frustrated. The environment feels punitive.
\end{tabular}




\section{EXAMINING TEACHERS' PARADIGM AND PEDAGOGY IN MANIFESTING CRITICAL HOPE IN LOW-INCOME MIDDLE SCHOOL}

Appendix D

Participant 4: Interview and Observation

\begin{tabular}{|c|c|c|}
\hline $\begin{array}{l}\text { Participant } \\
\text { P.4 5/24/19 }\end{array}$ & Questions & Comments \\
\hline Teacher & $\begin{array}{l}\text { Introduction: Thank you for agreeing } \\
\text { to participate in my study on Examining } \\
\text { Teachers' Paradigm and Pedagogy in } \\
\text { Manifesting Critical Hope in Low-Income } \\
\text { Middle School Cla ssrooms: A Narrative Inquiry } \\
\text { This interview will last approximately } \\
30 \text { to } 45 \text { minutes. I will be asking you to review } \\
\text { the transcript of the interview for accuracy. } \\
\text { 1. (PI's introduction to questions) } \\
\text { START WITH an OPEN-ENDED QUESTION: } \\
\text { JUST LIKE TELLING A STORY: We a lready } \\
\text { know through existing research that there is an } \\
\text { achievement gap between low-income students } \\
\text { and affluent students. Tell me, how do you } \\
\text { make your low-income students a spire to be } \\
\text { successful? } \\
\text { Follow-up question: Can you provide } \\
\text { an example? } \\
\text { 4. Why do you think on campus there } \\
\text { Follow-up questions to teacher's } \\
\text { 2. In your experience are you aware of } \\
\text { low expectations? }\end{array}$ & $\begin{array}{l}\text { 2. When they see other people from the } \\
\text { same place they are from that are successful, } \\
\text { bringing people outside the classroom and within } \\
\text { their community to speak and so they can see where } \\
\text { they have come from and where they started is in } \\
\text { the same neighborhood as them, and then they can } \\
\text { see that they can do this first hand. } \\
\text { 3. Lack of resources at home, or at school. } \\
\text { When they see things like old textbooks, or things } \\
\text { that are not as nice, maybe they feel it is a reflection } \\
\text { of them. Definitely a lack of resources. } \\
\quad 4 \text {. Some students before the school year } \\
\text { starts have a perceived notion and so they do not } \\
\text { even try. I try to get them out of a negative } \\
\text { mindset. They, their past, their failures don't define } \\
\text { them. }\end{array}$ \\
\hline
\end{tabular}


5. What learning opportunities do you provide your students that would strengthen their desire to be successful in the fields they are interested in?

Follow-up question: Do you ever have students who say that nothing positive happened to them over the weekend?

6. What issues or topics of conversation will be helpful for low-income students to see themselves as an active member of society?

7. How would you define hope?

8. (Based on your definition) How do you facilitate hope in your students

9. To what extent do you think being hopefulor having a spiration facilitates enga gement in your classroom?

Follow-up: What did you say?
5. I let them talk about things that are positive, I always have a starter on Mondays where they talk about 3 positive things that they did over the weekend. Even though this has nothing to do with the assignment, I want them to start off with a positive mindset.

Yes then I say, are you here right now? Do both yourlegs work? I know someone who is missing a leg. Did you eat today? Did you sleep somewhere? Did you take a hot shower? I keep trying to keep it basic.

6. Letting them tell their stories no matter what it is, if it is positive or negative. Let them know that they will not be judged.

7. Being positive. Always having something to look forward to. Always feeling like you can make a difference. Having a positive spirit. If you have nothing to look forward to, then you pretty much have no hope.

8. We talk about positive things. We share with our partners so they can hear how others feel. Then we share out so that we can hear others' passivity that may inspire others and creates a hopefulclimate.

9. I feel like if you do not have any hope you will not be engaged in school because you will not feel any value in what you are doing, but if you have hope you will try. Yesterday, a student ditched my class and I gave him detention. I thought he was going to hate me today because of some of the things I said, but today he shook my hand and said thank you.

I was going over the statistics on how 1 in 6 Latino males go to prison and how those who do not graduate often go to prison and I related that to him and his grades and his effort. I told him that I do not want one of my students go to prison, but it's on you. 
EXAMINING TEACHERS' PARADIGM AND PEDAGOGY IN MANIFESTING CRITICAL HOPE IN LOW-INCOME MIDDLE SCHOOL

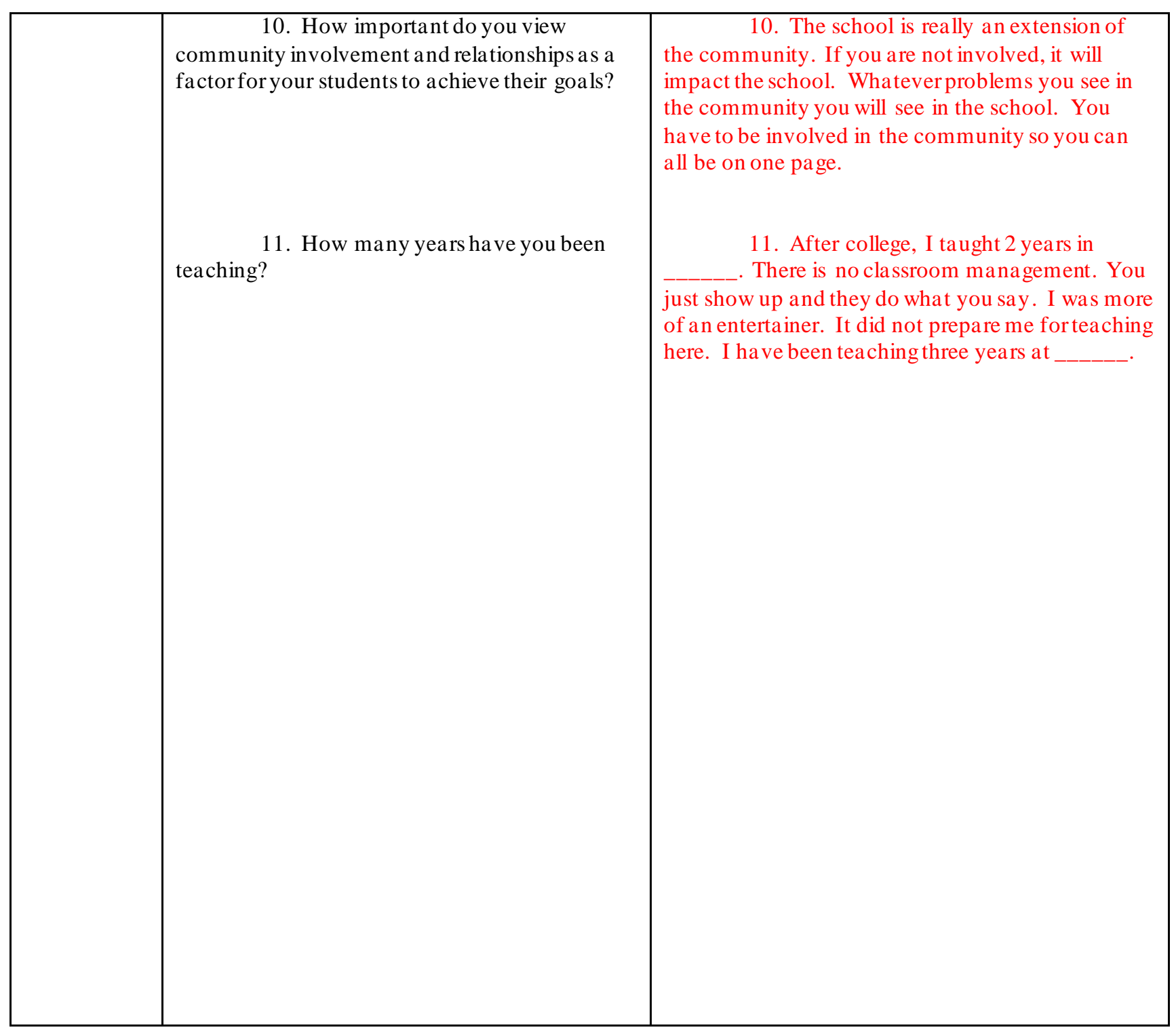


EXAMINING TEACHERS' PARADIGM AND PEDAGOGY IN MANIFESTING CRITICAL HOPE IN LOW-INCOME MIDDLE SCHOOL

\section{Manifestations of Critical Hope Reflected in Teachers' Paradigm and Pedagogy}

\section{Observation Matrix}

(For observing elements of Critical Hope manifested in teachers' language and behavior of hope, teachers' instructional delivery of hope, and teachers' lesson planning of hope)

\section{The Teacher \\ Time Observed:}

Observer:__D3____ Date: 1:28 5/23/18

\begin{tabular}{|c|c|c|c|c|}
\hline $\begin{array}{l}\text { Manifestations of } \\
\text { Critical Hope } \\
\text { Elements in } \\
\text { Teachers' } \\
\text { Paradigm \& } \\
\text { Pedagogy }\end{array}$ & $\begin{array}{l}\text { Manifestations } \\
\text { of Critical } \\
\text { Hope in: }\end{array}$ & $\begin{array}{l}\text { General } \\
\text { Indicators }\end{array}$ & Examples & Observed Evidence \\
\hline $\begin{array}{ll}\checkmark & \text { Goal Directed } \\
& \text { Thinking } \\
\checkmark & \text { Navigating } \\
& \text { Pathways } \\
\checkmark & \text { Willpower or } \\
& \text { Agency } \\
& \text { (Edwards, } \\
\text { Rand,Lopez, } \\
\text { \& Snyder, } \\
\text { 2007) }\end{array}$ & $\begin{array}{l}\text { Teachers use } \\
\text { of vocabulary } \\
\text { expression } \\
\text { and written } \\
\text { direction }\end{array}$ & 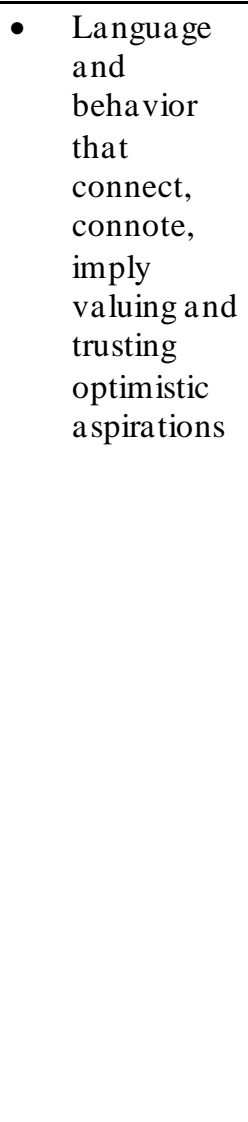 & $\begin{array}{ll}\circ & \begin{array}{l}\text { Positive } \\
\text { reinforcements }\end{array} \\
\circ & \text { Positive directives } \\
\circ & \text { Praises } \\
\circ & \text { Encouraging } \\
& \text { statements } \\
\circ & \text { Competence }\end{array}$ & $\begin{array}{l}\text { The last of the } \\
\text { questions are on your own. If } \\
\text { you need help, raise your hand. } \\
\text { When you are done, do not } \\
\text { pack up. We will share out. } \\
\text { Who does not have a } \\
\text { job? So share cropping is } \\
\text { sla very because they are not } \\
\text { getting paid. Who is going to } \\
\text { jail now? How are you doing? } \\
\text { Which one are you on? So now } \\
\text { you need a pass to get off the } \\
\text { plantation, so which } \\
\text { amendment would that be? } \\
\text { First step is go back and read } \\
\text { these amendments. If you do } \\
\text { not understand this you will not } \\
\text { understand the assignment. If } \\
\text { you get lost again raise your } \\
\text { hand. Yes, you got this. } \\
\text { Which one are you } \\
\text { stuck on? Get that stuff out of } \\
\text { your ear. } \\
\text { Let's do this together } \\
\text { then the rest are on your own. } \\
\text { Slavery is over now. Just } \\
\text { because we change a law does } \\
\text { that mean we change the }\end{array}$ \\
\hline
\end{tabular}




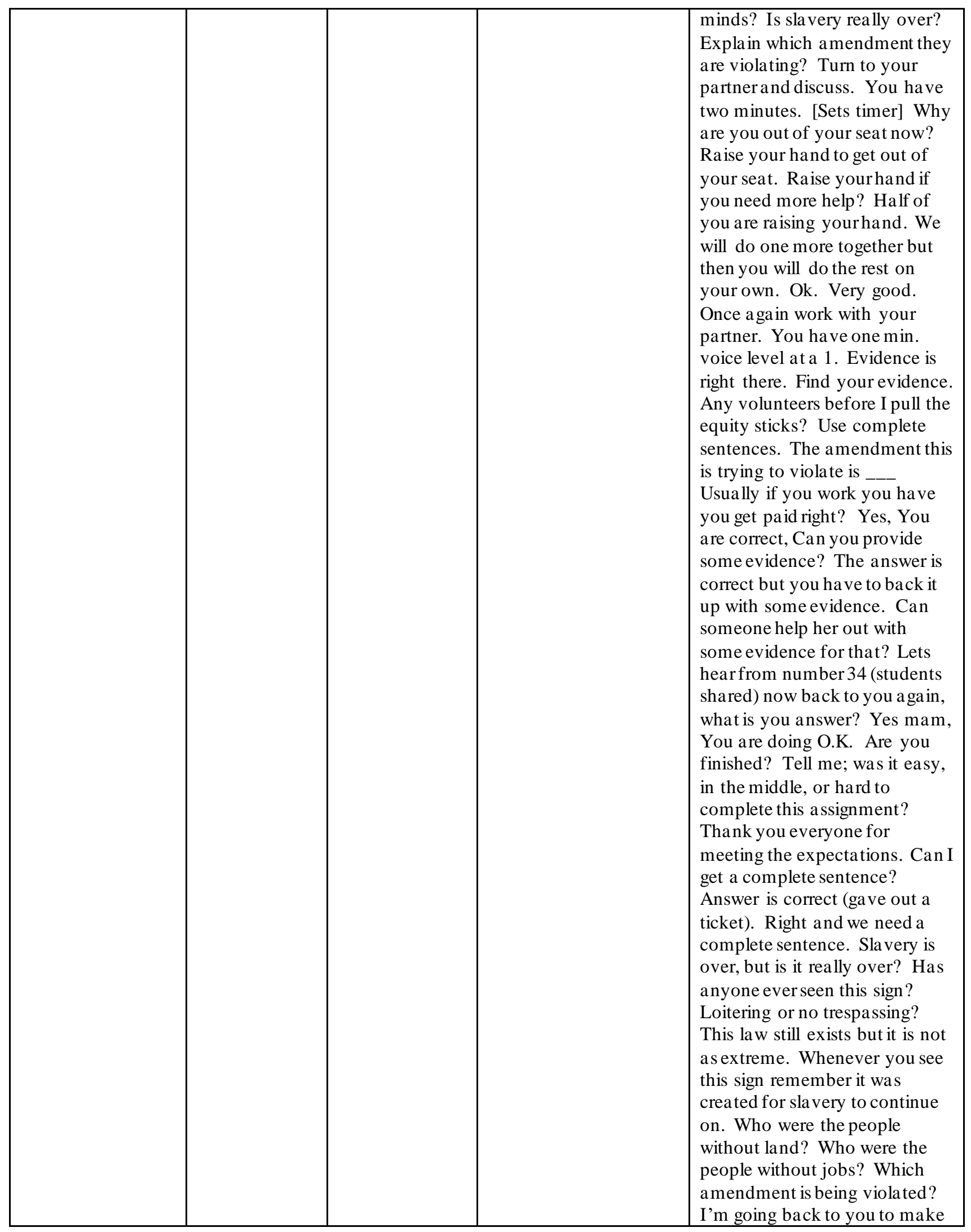




\begin{tabular}{|c|c|c|c|c|c|}
\hline & & & & & $\begin{array}{l}\text { sure you were listening. } \\
\text { Excellent. Thank you for giving } \\
\text { me a complete sentence, and for } \\
\text { giving me evidence. Yah, very } \\
\text { good. Raise yourhand if you } \\
\text { need a stamp and don't' leave } \\
\text { unless you get your permission } \\
\text { slip for him. }\end{array}$ \\
\hline $\begin{array}{l}\checkmark \\
\checkmark \\
\checkmark \\
\checkmark \\
\checkmark \\
\checkmark \\
\checkmark\end{array}$ & $\begin{array}{l}\text { Optimism } \\
\text { (Freire, 1997) } \\
\text { Ethics (Freire, } \\
\text { 1997) } \\
\text { Criticality } \\
\text { (Freire, 1974, } \\
\text { Giroux, 2011) } \\
\text { Social and } \\
\text { Historical } \\
\text { Consciousness } \\
\text { (Freire, 1974, } \\
\text { 1997) } \\
\text { Humanizing } \\
\text { Practices } \\
\text { (Freire, 1970, } \\
\text { 1974, 1994, } \\
\text { 1997) }\end{array}$ & $\begin{array}{l}\text { Teachers } \\
\text { prepared } \\
\text { written tasks } \\
\text { and } \\
\text { assignments }\end{array}$ & $\begin{array}{l}\text { - } \\
\text { Teacher } \\
\text { delivery in } \\
\text { prompting } \\
\text { critical } \\
\text { thinking } \\
\text { and } \\
\text { a spirations } \\
\text { to be caring } \\
\text { socially } \\
\text { conscious } \\
\text { and ethical. }\end{array}$ & $\begin{array}{ll} & \text { Higher levels of } \\
\text { questioning } \\
\text { Making value } \\
\text { judgments that } \\
\text { show caring and } \\
\text { ethical disposition } \\
\text { based upon the } \\
\text { criteria }\end{array}$ & $\begin{array}{l}\text { Explain which } \\
\text { amendment they are trying to } \\
\text { violate. Even though they } \\
\text { passed these laws, now they are } \\
\text { trying to find ways to go around } \\
\text { them. The grandfather cla use. } \\
\text { Which amendment are they } \\
\text { trying to violate? Is slavery } \\
\text { really over? }\end{array}$ \\
\hline $\begin{array}{l}\checkmark \\
\checkmark \\
\checkmark\end{array}$ & $\begin{array}{l}\text { Sense of } \\
\text { Community } \\
\text { (hooks, 2003, } \\
\text { Freire, 1997) } \\
\text { Caring } \\
\text { Relationships } \\
\text { (Noddings, } \\
\text { 2017, Freire } \\
\text { 1997) } \\
\text { Knowledge of } \\
\text { Social \& } \\
\text { Cultural } \\
\text { Capital } \\
\text { (Bourdieu, } \\
\text { 1986) } \\
\text { Critical } \\
\text { Citizenry } \\
\text { (Giroux, 2011, } \\
\text { Freire, 1997) } \\
\text { Democratic } \\
\text { Advocacy } \\
\text { (Giroux, 2011) }\end{array}$ & $\begin{array}{l}\text { Teachers } \\
\text { composed } \\
\text { lesson plans }\end{array}$ & $\begin{array}{l}\text { Teacher } \\
\text { planning } \\
\text { that may } \\
\text { elicit a } \\
\text { sense of } \\
\text { community } \\
\text { and } \\
\text { humanizing } \\
\text { practices. }\end{array}$ & $\begin{array}{l}\text { Lesson plans that } \\
\text { requires } \\
\text { collaboration and } \\
\text { creativity in } \\
\text { manifesting voice } \\
\text { and advocacy that } \\
\text { reflect sense of } \\
\text { community and } \\
\text { humanizing } \\
\text { practices }\end{array}$ & \\
\hline & & \multicolumn{4}{|c|}{$\begin{array}{l}\text { Other observations: Teacher is constantly walking around the room supporting } \\
\text { and checking in on each scholar. The room is set up in rows with pairs of two. Teacher is } \\
\text { very respectful with the students. You can feel his presence; there is care and high } \\
\text { expectations. He is relentless asking for evidence, complete sentences, and for work }\end{array}$} \\
\hline
\end{tabular}


completion. This is an 8th grade History class. The environment is very cultural. There are flags all up around the room from different countries. America, Mexico, Africa, England, China, and so on. There are posters of Fredrick Douglass, Martin Luther King, and Obama on the front wall. Lot of students work up on the back wall. Posters on group NORMS, AVID NORMS, and the 5C's paragraph writing sentence frame. He is constantly questioning them, getting them to think, and relating the historical content to relevant things they experience in their daily life. 
EXAMINING TEACHERS' PARADIGM AND PEDAGOGY IN MANIFESTING CRITICAL
HOPE IN LOW-INCOME MIDDLE SCHOOL

Appendix E

Participant 5: Interview and Observation

\begin{tabular}{|c|c|c|}
\hline $\begin{array}{l}\text { Participant } \\
\text { P.5 5/24/19 }\end{array}$ & Questions & Comments \\
\hline Teacher & $\begin{array}{l}\text { Introduction: Thank you for } \\
\text { agreeing to participate in my study on } \\
\text { Examining Teachers' Paradigm and Pedagogy } \\
\text { in Manifesting Critical Hope in Low-Income } \\
\text { Middle School Classrooms: A Narrative } \\
\text { Inquiry } \\
\text { This interview will last } \\
\text { approximately } 30 \text { to } 45 \text { minutes. I will be } \\
\text { a sking you to review the transcript of the } \\
\text { interview for accuracy. } \\
\text { 1. (PI's introduction to questions) } \\
\text { START WITH an OPEN-ENDED } \\
\text { QUESTION: JUST LIKE TELLING A } \\
\text { STORY: We already know through existing } \\
\text { research that there is an achievement gap } \\
\text { between low-income students and affluent } \\
\text { students. Tell me, how do you make your } \\
\text { low-income students a spire to be successful? } \\
\text { Follow-up question: Can you provide } \\
\text { an example? }\end{array}$ & $\begin{array}{l}\text { 1. I would say I inspire my low-income } \\
\text { students to be successful by first; I try to be a model, } \\
\text { an example to them. Throughout the year I tell them } \\
\text { my own personalstories that relate to them. How I } \\
\text { got to this point. How I made my way. Things were } \\
\text { not always set up for me to be successful and through } \\
\text { my family and my faith I made myself successful. } \\
\text { That they can do that too. Right now they might not } \\
\text { have a lot of control of their circumstances because } \\
\text { they are minors. } \\
\text { They have to keep in mind if they want to } \\
\text { achieve it, if they want to do it, they can. They have } \\
\text { to keep this in mind in order to achieve. } \\
\text { 2. For the most part, I try to talk to my } \\
\text { students and when they do open up and tell me, } \\
\text { usually it is a sad story they tell me. I instantly give } \\
\text { them a compliment. Do you know how amazing you } \\
\text { are because you have gone through that? Do you } \\
\text { know how many adults have not even experienced } \\
\text { this? Because you are dealing with this now, you are } \\
\text { making yourself stronger than the one who has never } \\
\text { experienced this in life. No matter what their story is, } \\
\text { or how bad it is, I let them know you can turn that } \\
\text { around and use that same energy and same force to } \\
\text { push you forward. Then one day you can teach } \\
\text { someone else how to overcome difficulty. I feel often } \\
\text { that when kids feel they were abused, molested, or } \\
\text { when they were given up for adoption, then they can } \\
\text { use that as an excuse, like you do not have to try } \\
\text { anymore. I try to use this as a story to turn it around }\end{array}$ \\
\hline
\end{tabular}




\section{EXAMINING TEACHERS' PARADIGM AND PEDAGOGY IN MANIFESTING CRITICAL HOPE IN LOW-INCOME MIDDLE SCHOOL}

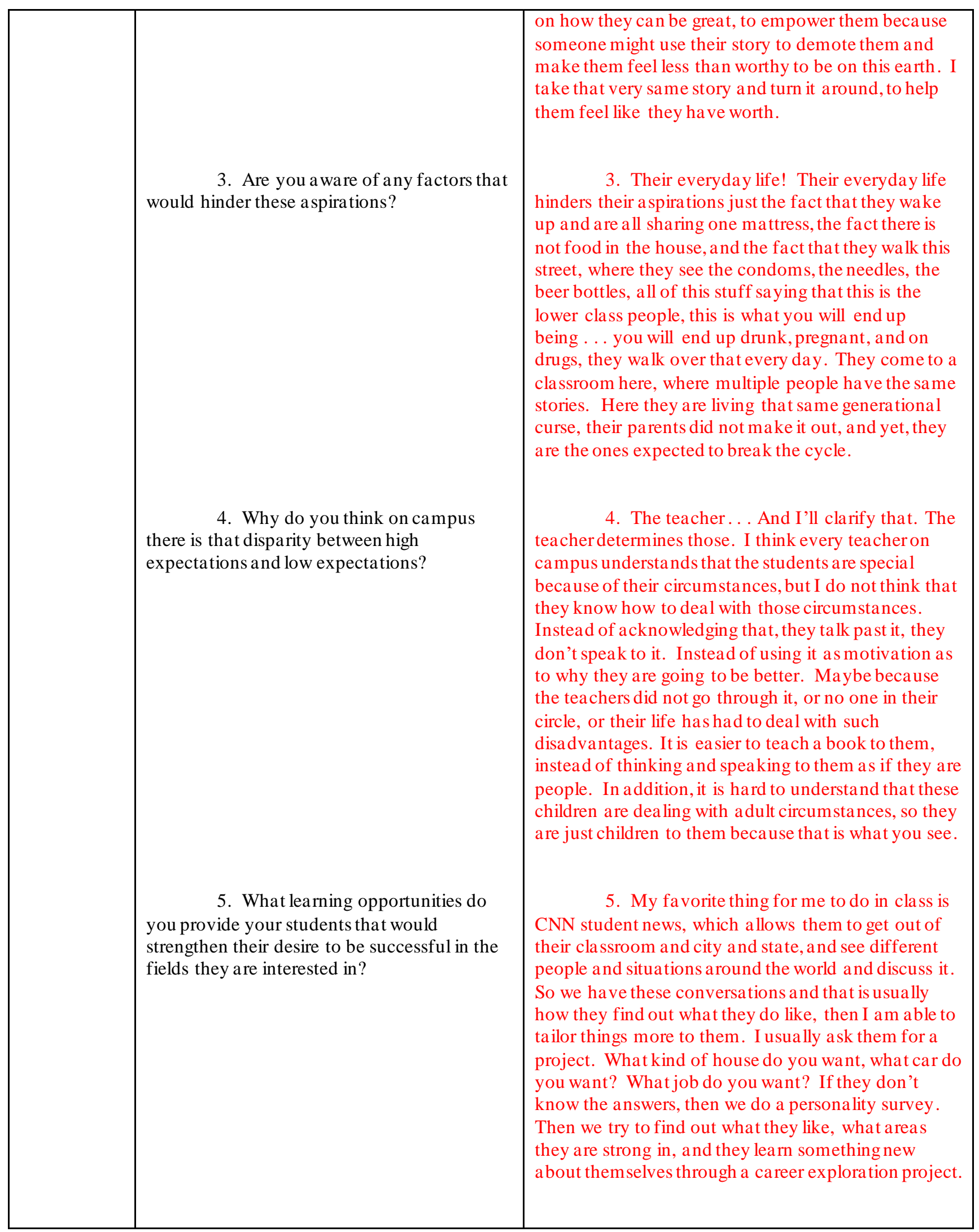




\section{EXAMINING TEACHERS' PARADIGM AND PEDAGOGY IN MANIFESTING CRITICAL HOPE IN LOW-INCOME MIDDLE SCHOOL}

\begin{tabular}{|c|c|}
\hline $\begin{array}{l}\text { 6. What issues or topics of } \\
\text { conversation will be helpful for low-income } \\
\text { students to see themselves as an active } \\
\text { member of society? }\end{array}$ & $\begin{array}{l}\text { 6. Politics, some of my students are so } \\
\text { entrenched in their own neighborhood and their own } \\
\text { culture that they do not really understand what is } \\
\text { going on around them. I love it that they embrace } \\
\text { their culture and their community, and it is a rude } \\
\text { a wakening when you get outside your community and } \\
\text { someone puts you in your place somewhere. I was } \\
\text { always told what my place was in the world; I have } \\
\text { worked to rise above it, but I was reminded of whom I } \\
\text { am. For example, one time my administrator said to } \\
\text { me what my people signify in the status quo of culture } \\
\text { and my community. As much as I want them to keep } \\
\text { their innocence and their culture, they also have to } \\
\text { know they have to have a voice because someone is } \\
\text { going to try to burst your bubble someday. You must } \\
\text { know how to respond in these situations. If you don't } \\
\text { tell them, they will go out and set themselves up for } \\
\text { failure and get knocked off of their journey. It will } \\
\text { take them a while to come back. They need to know } \\
\text { how others see what your place is in society and how } \\
\text { you have to work against that place, and if no one } \\
\text { ever tells you, you do not know what you are working } \\
\text { against. }\end{array}$ \\
\hline 7. How would you define hope? & $\begin{array}{l}\text { 7. Believing in the unseen (laughter). My } \\
\text { hope is my faith. I really don't say hope, I say faith. }\end{array}$ \\
\hline $\begin{array}{l}\text { 8. (Based on your definition) How } \\
\text { do you facilitate hope in your students }\end{array}$ & $\begin{array}{l}\text { 8. Demonstration first. I have to have faith, } \\
\text { so I have to have hope in them that I have confidence } \\
\text { in them, in their abilities first because a lot of them do } \\
\text { not believe in themselves. I have to have almost an } \\
\text { exaggerated amount of hope, so they buy in. Even } \\
\text { when they do something small, I have to show them } \\
\text { and make it a big deal. Wow!!! This is a mazing!! } \\
\text { Wow!! Then after a few months they start using my } \\
\text { language and I have to make sure that my jar of hope } \\
\text { is full and running over because they might not see } \\
\text { hope in their homes, or on the street. I have to be that } \\
\text { demonstration, that ray of light, when everyone who } \\
\text { is around them is telling them something different. }\end{array}$ \\
\hline $\begin{array}{l}\text { 9. To what extent do you think being } \\
\text { hopefulor having a spiration facilitates } \\
\text { engagement in your classroom? }\end{array}$ & $\begin{array}{l}\text { 9. Oh that's, everything OMG. (Laughter) I } \\
\text { don't even know how to say that in another way. } \\
\text { They have to know that someone is in their corner and } \\
\text { that someone is expecting them to be successful. } \\
\text { They see that you are relentless, that you are not } \\
\text { giving up, that they have no other option, than to meet } \\
\text { that standard. You have to be hopefuland confident } \\
\text { in yourability as a teacher. Just like if I need a heart } \\
\text { transplant. I'm not going to get any doctor, I need to } \\
\text { see you are together, in need to see your interaction } \\
\text { with your family, I need to have confidence in you, or }\end{array}$ \\
\hline
\end{tabular}




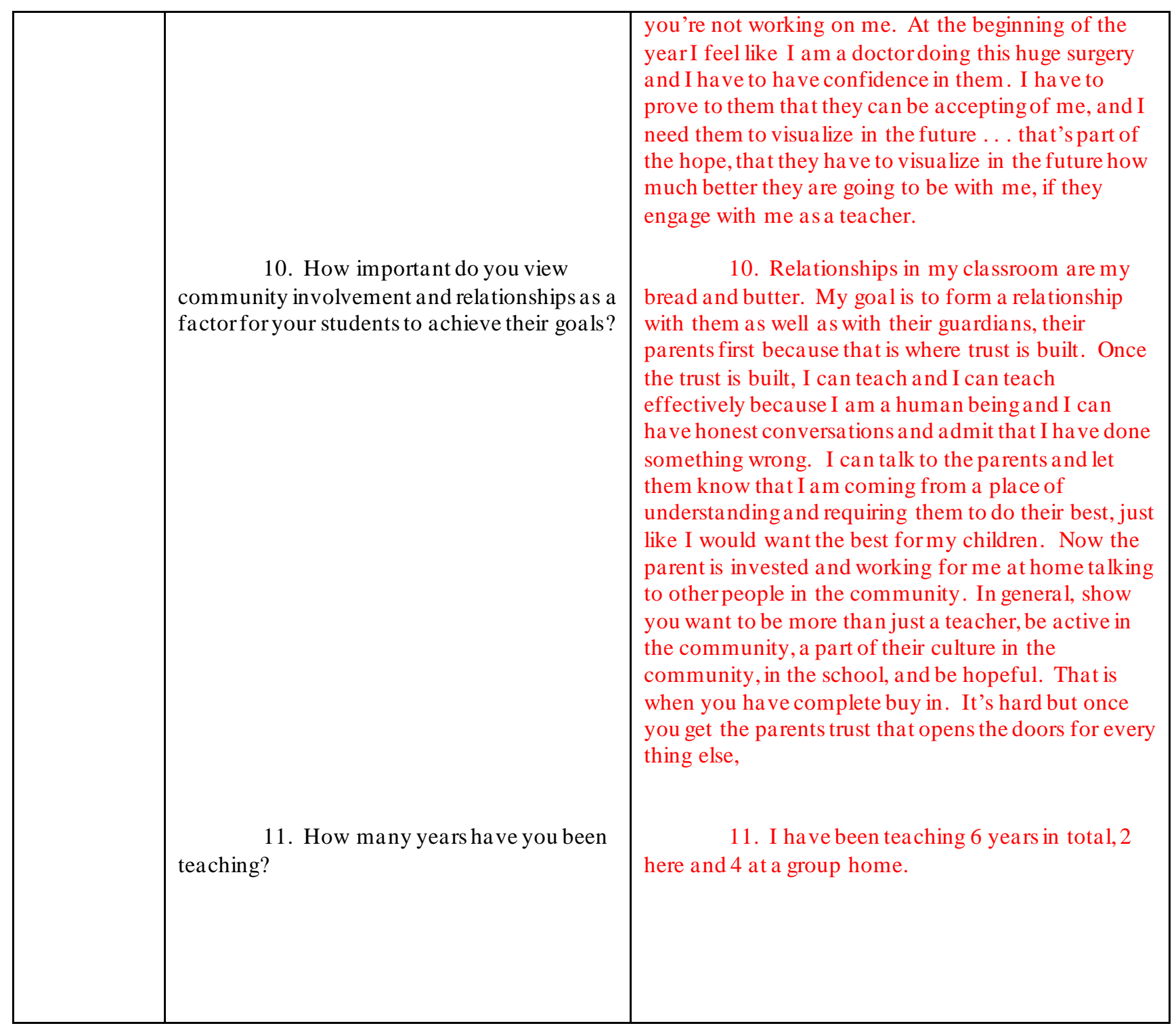




\section{Manifestations of Critical Hope Reflected in Teachers' Paradigm and Pedagogy}

\section{Observation Matrix}

(For observing elements of Critical Hope manifested in teachers' language and behavior of hope, teachers' instructional delivery of hope, and teachers' lesson planning of hope)

\section{The Teacher}

Time Observed: $\quad$ P-5
Observer:
$\mathrm{C} 2$
Date: 1:00 5/24/19

\begin{tabular}{|c|c|c|c|c|}
\hline $\begin{array}{l}\text { Manifestations of } \\
\text { Critical Hope } \\
\text { Elements in } \\
\text { Teachers' } \\
\text { Paradigm \& } \\
\text { Pedagogy } \\
\end{array}$ & $\begin{array}{l}\text { Manifestations } \\
\text { of Critical Hope } \\
\text { in: }\end{array}$ & $\begin{array}{l}\text { General } \\
\text { Indicators }\end{array}$ & Examples & Observed Evidence \\
\hline 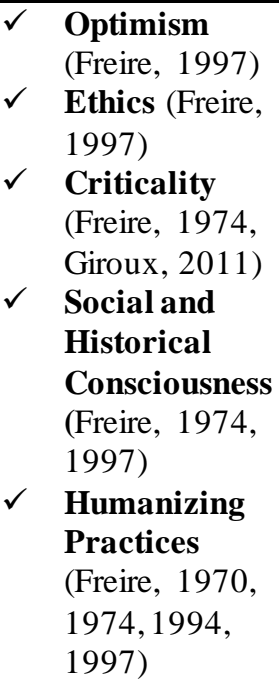 & $\begin{array}{l}\text { Teachers } \\
\text { prepared } \\
\text { written tasks } \\
\text { and } \\
\text { assignments }\end{array}$ & $\begin{array}{l}\text { Teacher } \\
\text { delivery in } \\
\text { prompting } \\
\text { critical } \\
\text { thinking and } \\
\text { a spirations } \\
\text { to be caring } \\
\text { socially } \\
\text { conscious } \\
\text { and ethical. }\end{array}$ & $\begin{array}{ll}\text { Higher } \\
\text { levels of } \\
\text { questioning } \\
\text { Making } \\
\text { value } \\
\text { judgments } \\
\text { that show } \\
\text { caring and } \\
\text { ethical } \\
\text { disposition } \\
\text { based upon } \\
\text { the criteria }\end{array}$ & \\
\hline $\begin{array}{ll}\checkmark & \begin{array}{l}\text { Sense of } \\
\text { Community } \\
\text { (hooks, 2003, }\end{array} \\
& \text { Freire, 1997) } \\
\text { Caring } \\
\text { Relationships } \\
\text { (Noddings, } \\
2017, \text { Freire } \\
1997 \text { ) } \\
\checkmark \\
\text { Knowledge of }\end{array}$ & $\begin{array}{l}\text { Teachers } \\
\text { composed } \\
\text { lesson plans }\end{array}$ & $\begin{array}{l}\text { Teacher } \\
\text { planning that } \\
\text { may elicit a } \\
\text { sense of } \\
\text { community } \\
\text { and } \\
\text { humanizing } \\
\text { practices. }\end{array}$ & $\begin{array}{l}\text { Lesson plans } \\
\text { that requires } \\
\text { collaboration } \\
\text { and } \\
\text { creativity in } \\
\text { manifesting } \\
\text { voice and } \\
\text { advocacy } \\
\text { that reflect } \\
\text { sense of } \\
\end{array}$ & \\
\hline
\end{tabular}




\begin{tabular}{|c|c|c|c|c|}
\hline$\checkmark$ & $\begin{array}{l}\text { Social \& } \\
\text { Cultural } \\
\text { Capital } \\
\text { (Bourdieu, } \\
\text { 1986) } \\
\text { Critical } \\
\text { Citizenry } \\
\text { (Giroux, 2011, } \\
\text { Freire, 1997) } \\
\text { Democratic } \\
\text { Advocacy } \\
\text { (Giroux, 2011) }\end{array}$ & & $\begin{array}{l}\text { community } \\
\text { and } \\
\text { humanizing } \\
\text { practices }\end{array}$ & \\
\hline & & \multicolumn{3}{|c|}{$\begin{array}{l}\text { Other observations: The room is extremely cultural. There are positive quotes } \\
\text { on the walls. A Norms poster. The books that the students are choosing to read are all } \\
\text { multicultural-leveled readers. This is an ELD class. There is a hand-written poster on } \\
\text { the wall that says, "ELD is not about speaking good or bad it's about learning different } \\
\text { dialects." The teacher is strong, caring, and sets high expectations. She walked around } \\
\text { the room and checked in on each student. She holds them accountable and they } \\
\text { respond to her. They want her approval. The room is set up in collaborative } \\
\text { groupings. Each grouping has a different color tape on the floor to identify the table } \\
\text { groups. While I was in there, she asked her aid to watch the class so she could speak to } \\
\text { me outside. She broke down in tears because she was just informed that she would not } \\
\text { be teaching ELD next year. She loves her ELD students and she was excited about } \\
\text { getting better and being more prepared to meet their needs in the upcoming school } \\
\text { year. When we came back inside a student made a comment. Mrs. ----- we want } \\
\text { you to be happy. It touched my heart. They noticed that she as upset. }\end{array}$} \\
\hline
\end{tabular}




\section{EXAMINING TEACHERS' PARADIGM AND PEDAGOGY IN MANIFESTING CRITICAL HOPE IN LOW-INCOME MIDDLE SCHOOL}

\section{Appendix F}

\section{Participant 6: Interview and Observation}

\begin{tabular}{|c|c|c|}
\hline $\begin{array}{l}\text { Participant } \\
\text { P.6 5/29/19 }\end{array}$ & Questions & Comments \\
\hline Teacher & $\begin{array}{l}\text { Introduction: Thank you for } \\
\text { a greeing to participate in my study on } \\
\text { Examining Teachers' Paradigm and Pedagogy } \\
\text { in Manifesting Critical Hope in Low-Income } \\
\text { Middle School Classrooms: A Narrative } \\
\text { Inquiry } \\
\text { This interview will last } \\
\text { approximately } 30 \text { to } 45 \text { minutes. I will be } \\
\text { asking you to review the transcript of the } \\
\text { interview for accuracy. } \\
\text { 1. (PI's introduction to questions) } \\
\text { START WITH an OPEN-ENDED } \\
\text { QUESTION: JUST LIKE TELLING A } \\
\text { STORY: We already know through existing } \\
\text { research that there is an achievement gap } \\
\text { between low-income students and affluent } \\
\text { students. Tell me, how do you make your } \\
\text { low-income students a spire to be successful? } \\
\text { Follow-up question: When you say } \\
\text { your belief, do you mean your belief as the } \\
\text { teacher, or the scholars' belief in themselves? } \\
\text { Follow-up questions to teacher's } \\
\text { 2. In your experience are you aware } \\
\text { storytelling: } \\
\text { of any factors that compliment students' desire } \\
\text { to be successful? }\end{array}$ & $\begin{array}{l}\text { 1. By giving them the support and building } \\
\text { confidence in them that they can achieve whatever } \\
\text { they put their mind into. Positive reinforcement. No } \\
\text { opt out. When they say I don't know, IDK, letting } \\
\text { them not be afraid to try. Even if the answer is not } \\
\text { correct, showing them that they can learn the } \\
\text { information whether it is at this school, or another } \\
\text { school. Your belief is what builds that confidence in } \\
\text { that scholar. } \\
\qquad \text { Me believing in them, and them believing in } \\
\text { themselves. My belief in them, so it translates into } \\
\text { them believing in themselves. } \\
\text { 2. Umm, gosh, um. Couple things. One } \\
\text { daily attendance, coming to school and wanting to do } \\
\text { well, but the second part is a sking questions when } \\
\text { they feel they do not understand the materialin a way } \\
\text { to help them feel comfortable in the classroom. To } \\
\text { me the environment is very important staying positive } \\
\text { to help expand their effort. } \\
\text { 3. Being negative in class, not listening to } \\
\text { the scholars if they have questions or just to be heard } \\
\text { sometimes. They know real quickly if you don't care, } \\
\text { or have an attitude that will hinder them in a negative } \\
\text { way. }\end{array}$ \\
\hline
\end{tabular}




\section{EXAMINING TEACHERS' PARADIGM AND PEDAGOGY IN MANIFESTING CRITICAL HOPE IN LOW-INCOME MIDDLE SCHOOL}

expectations and low expectations?

5. What learning opportunities do you provide your students that would strengthen their desire to be successful in the fields they are interested in?

6. What issues or topics of conversation will be helpful for low-income students to see themselves as an active member of society?

7. How would you define hope?

8. (Based on your definition) How do you facilitate hope in your students

9. To what extent do you think being hopefulor having a spiration facilitates engagement in your cla ssroom?

10. How important do you view community involvement and relation ships as a factor for your students to achieve their goals? expectation might seem to be low. If they understand from the teachers and staff that we expect a level of achievement of effort most scholars will demand more of themselves than the expectations of the teachers or the staff. Not afraid to challenge scholars even when they say they can't do it even though you know they can.

5. Learning opportunities would include TPS, Google classroom, videos, discussions; with these different a venues there are no two days a like in my class and it keeps the students on their toes in wanting to try to continuously to do well or better and they don't feel like it is repetition in a history class, the same thing over and over like read and answer questions.

6. Not to be afraid to use your voice when we have discussions. Its ok to have an opinion, but make sure with that opinion you have facts with it and just because you are 13 or 14 does not mean that you cant have an impact and to think a bout voting, community service, help with siblings, school work or homework, anything that would help in your community so you can see the positive impact of giving. It feels good like they have accomplished something. It usually brings a smile and they are proud of that achievement.

7. To believe in and never giving up.

8. That I never give up on them, even if we have a bad day; that is just one day. Tomorrow will be a new day. Even if they give up on themselves, that I never do or will give up, that I a m always on their side.

9. Um, usually being in a positive environment and with that you get enga gement with most scholars whether they truly want to raise their hand or not, or you use cold call, so they give an answer and they try their best knowing they will not be put down, or made fun of.

10. I find it important for the community or the school community or outside the community; by giving them positive reinforcement most students will take that positive reinforcement and use it. The more 
EXAMINING TEACHERS' PARADIGM AND PEDAGOGY IN MANIFESTING CRITICAL HOPE IN LOW-INCOME MIDDLE SCHOOL

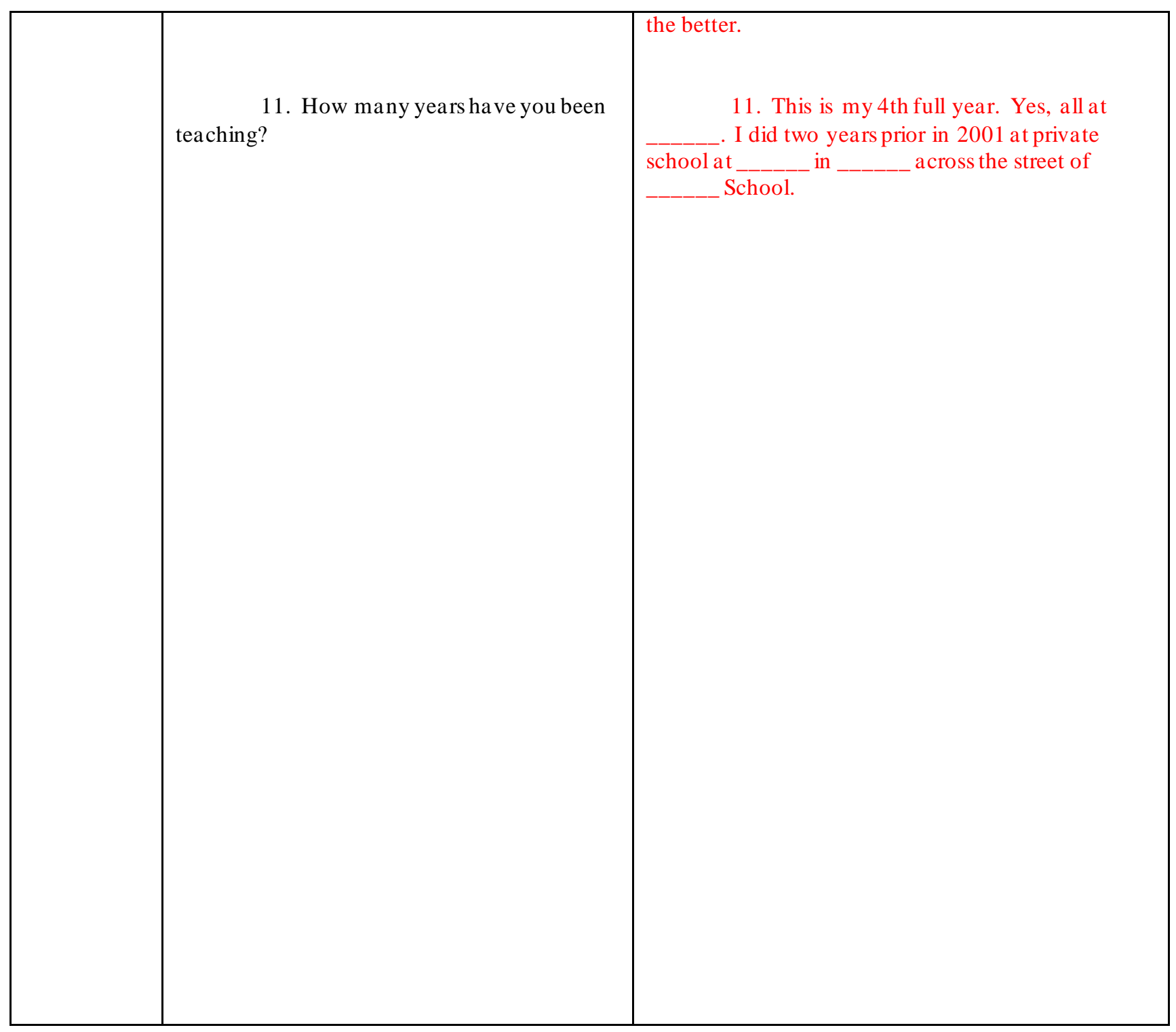


EXAMINING TEACHERS' PARADIGM AND PEDAGOGY IN MANIFESTING CRITICAL HOPE IN LOW-INCOME MIDDLE SCHOOL

\section{Manifestations of Critical Hope Reflected in Teachers' Paradigm and Pedagogy}

\section{Observation Matrix}

(For observing elements of Critical Hope manifested in teachers' language and behavior of hope, teachers' instructional delivery of hope, and teachers' lesson planning of hope)

\section{The Teacher}

Time Observed: $\_$P-6
Observer: _ G5
Date: 10:02am 5/30/19

\begin{tabular}{|c|c|c|c|c|}
\hline $\begin{array}{l}\text { Manifestations of } \\
\text { Critical Hope } \\
\text { Elements in } \\
\text { Teachers' } \\
\text { Paradigm \& } \\
\text { Pedagogy }\end{array}$ & $\begin{array}{l}\text { Manifestations } \\
\text { of Critical } \\
\text { Hope in: }\end{array}$ & $\begin{array}{l}\text { General } \\
\text { Indicators }\end{array}$ & Examples & Observed Evidence \\
\hline $\begin{array}{ll}\checkmark & \text { Goal Directed } \\
& \text { Thinking } \\
\checkmark & \text { Navigating } \\
& \text { Pathways } \\
\checkmark & \text { Willpower or } \\
& \text { Agency } \\
& \text { (Edwards, } \\
\text { Rand, Lopez, } \\
\text { \& Snyder, } \\
\text { 2007) }\end{array}$ & $\begin{array}{l}\text { Teachers use } \\
\text { of vocabulary } \\
\text { expression } \\
\text { and written } \\
\text { direction }\end{array}$ & $\begin{array}{l}\text { Language } \\
\text { and } \\
\text { behavior } \\
\text { that } \\
\text { connect, } \\
\text { connote, } \\
\text { imply } \\
\text { valuing } \\
\text { and } \\
\text { trusting } \\
\text { optimistic } \\
\text { aspirations }\end{array}$ & $\begin{array}{cl}\circ & \begin{array}{l}\text { Positive } \\
\text { reinforcements }\end{array} \\
\circ & \begin{array}{l}\text { Positive } \\
\text { directives }\end{array} \\
\circ & \text { Praises } \\
\circ & \text { Encouraging } \\
& \text { statements } \\
\circ & \text { Competence }\end{array}$ & $\begin{array}{l}\text { When I walked in, } \\
\text { students were sitting quietly } \\
\text { watching the ending to the movie } \\
\text { Glory. Teacherhad sent home } \\
\text { permission slips last week since } \\
\text { the movie is rated R. Teacher } \\
\text { stops the movie to engage the } \\
\text { students in a conversation. } \\
\text { "Remember how he was } \\
\text { wounded the day before and he } \\
\text { has been marching continuously } \\
\text { for a few days now, so he } \\
\text { probably was light headed, } \\
\text { fatigued." Students are } \\
\text { interacting with him. Then he } \\
\text { finishes up the film for the next } \\
\text { 15 minutes of class. He hands } \\
\text { the guided questions to a student } \\
\text { and a sks him to pass it out to the } \\
\text { class. He a sks another student to } \\
\text { turn on the lights. Enga ges the } \\
\text { class in a conversation. "How } \\
\text { many of us enjoyed the movie?" } \\
\text { Students are raising their hands. } \\
\text { "How did this movie make you } \\
\text { feel? Is this movie a true story?" } \\
\text { "Yes, it is based off a true story } \\
\text { during the Civil War." Teacher }\end{array}$ \\
\hline
\end{tabular}




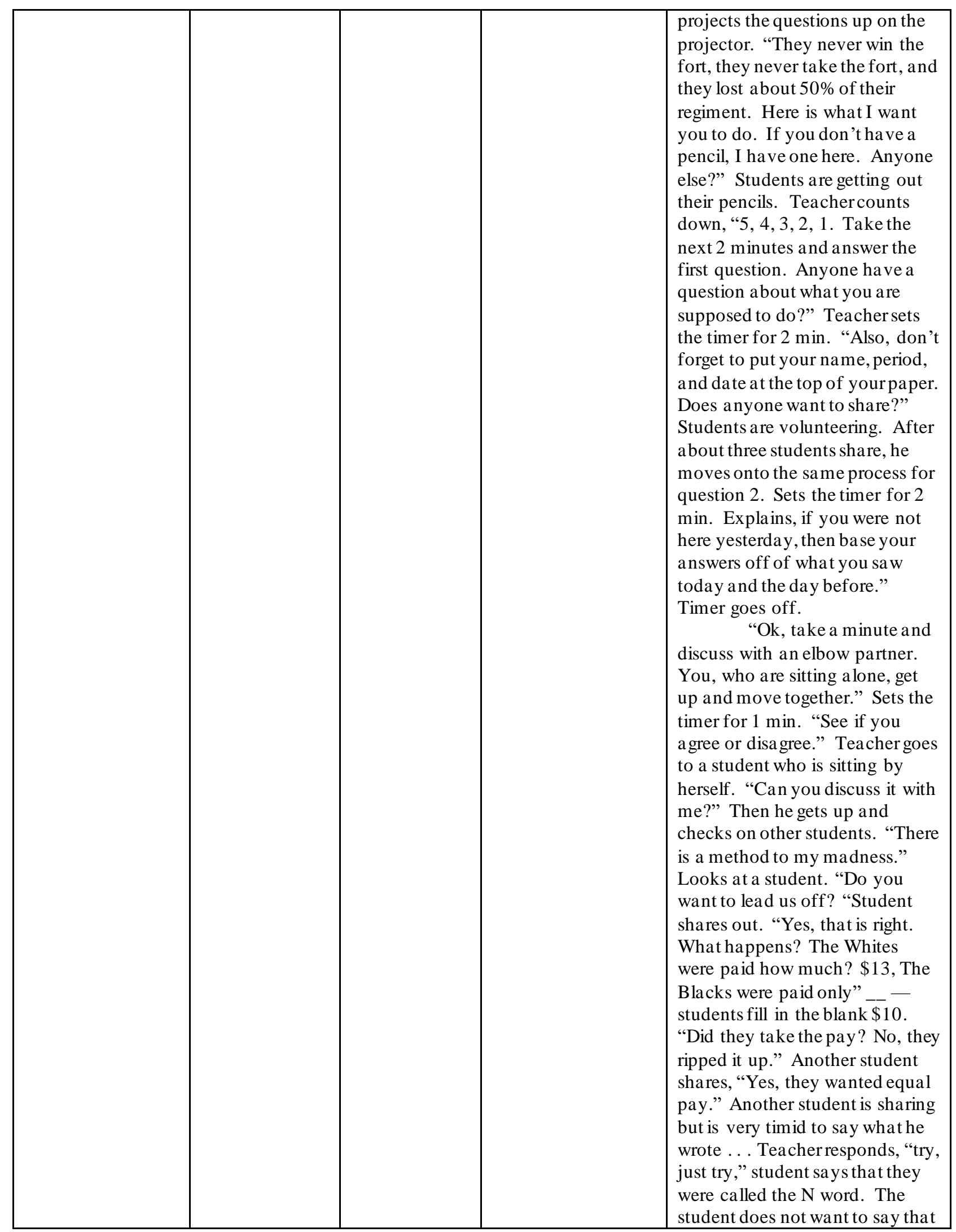




\begin{tabular}{|c|c|c|c|c|}
\hline & & & & $\begin{array}{l}\text { word. Teacher affirms him. } \\
\text { Yes, then another student shares } \\
\text { out. "AWE, yes! What about } \\
\text { the point of the shoes and } \\
\text { socks?" Teacheris constantly } \\
\text { enga ging students in dia logue } \\
\text { through questioning. "He saw } \\
\text { his feet, pretty disgusting right? } \\
\text { What does Colonial Shaw do?" } \\
\text { Teacher continues this } \\
\text { process. Moves onto question } 3 \\
\text { and sets the timer. "I need a } \\
\text { pause; someone asked a good } \\
\text { question here. I need a time out. } \\
\text { What does this mean, "turning } \\
\text { point"? Let's make an } \\
\text { educational guess here." He } \\
\text { calls on students to pull out the } \\
\text { meaning of the phrase. "Are you } \\
\text { saying changes? Ok Good! } \\
\text { Let's use your words instead, } \\
\text { What changed to make Colonial } \\
\text { Shaw ...? Excellent question! } \\
\text { When in doubt, ask!!"” }\end{array}$ \\
\hline $\begin{array}{ll}\checkmark & \text { Optimism } \\
& \text { (Freire, 1997) } \\
\checkmark & \text { Ethics (Freire, } \\
& \text { 1997) } \\
\checkmark & \text { Criticality } \\
& \text { (Freire, 1974, } \\
& \text { Giroux, 2011) } \\
\checkmark & \text { Social and } \\
& \text { Historical } \\
& \text { Consciousness } \\
& \text { (Freire, 1974, } \\
& \text { 1997) } \\
\checkmark & \text { Humanizing } \\
& \text { Practices } \\
& \text { (Freire, 1970, } \\
\text { 1974, 1994, } \\
\text { 1997) }\end{array}$ & $\begin{array}{l}\text { Teachers } \\
\text { prepared } \\
\text { written tasks } \\
\text { and } \\
\text { assignments }\end{array}$ & 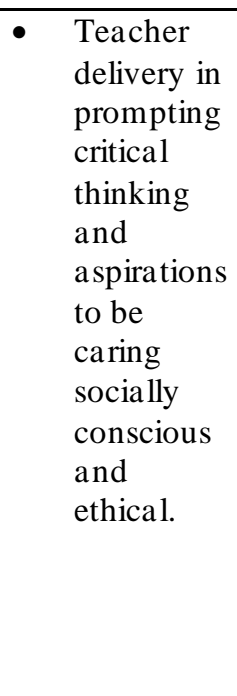 & $\begin{array}{ll} & \text { Higher levels of } \\
\text { questioning } & \text { Making value } \\
\text { judgments that } \\
\text { show caring and } \\
\text { ethical } \\
\text { disposition } \\
\text { based upon the } \\
\text { criteria }\end{array}$ & \\
\hline 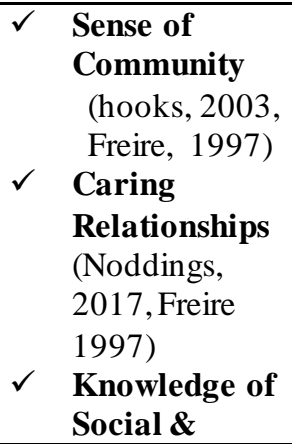 & $\begin{array}{l}\text { Teachers } \\
\text { composed } \\
\text { lesson plans }\end{array}$ & $\begin{array}{l}\text { Teacher } \\
\text { planning } \\
\text { that may } \\
\text { elicit a } \\
\text { sense of } \\
\text { community } \\
\text { and } \\
\text { humanizing } \\
\text { practices. }\end{array}$ & $\begin{array}{l}\text { Lesson plans } \\
\text { that requires } \\
\text { collaboration } \\
\text { and creativity in } \\
\text { manifesting } \\
\text { voice and } \\
\text { advocacy that } \\
\text { reflect sense of } \\
\text { community and } \\
\text { humanizing } \\
\text { practices }\end{array}$ & \\
\hline
\end{tabular}




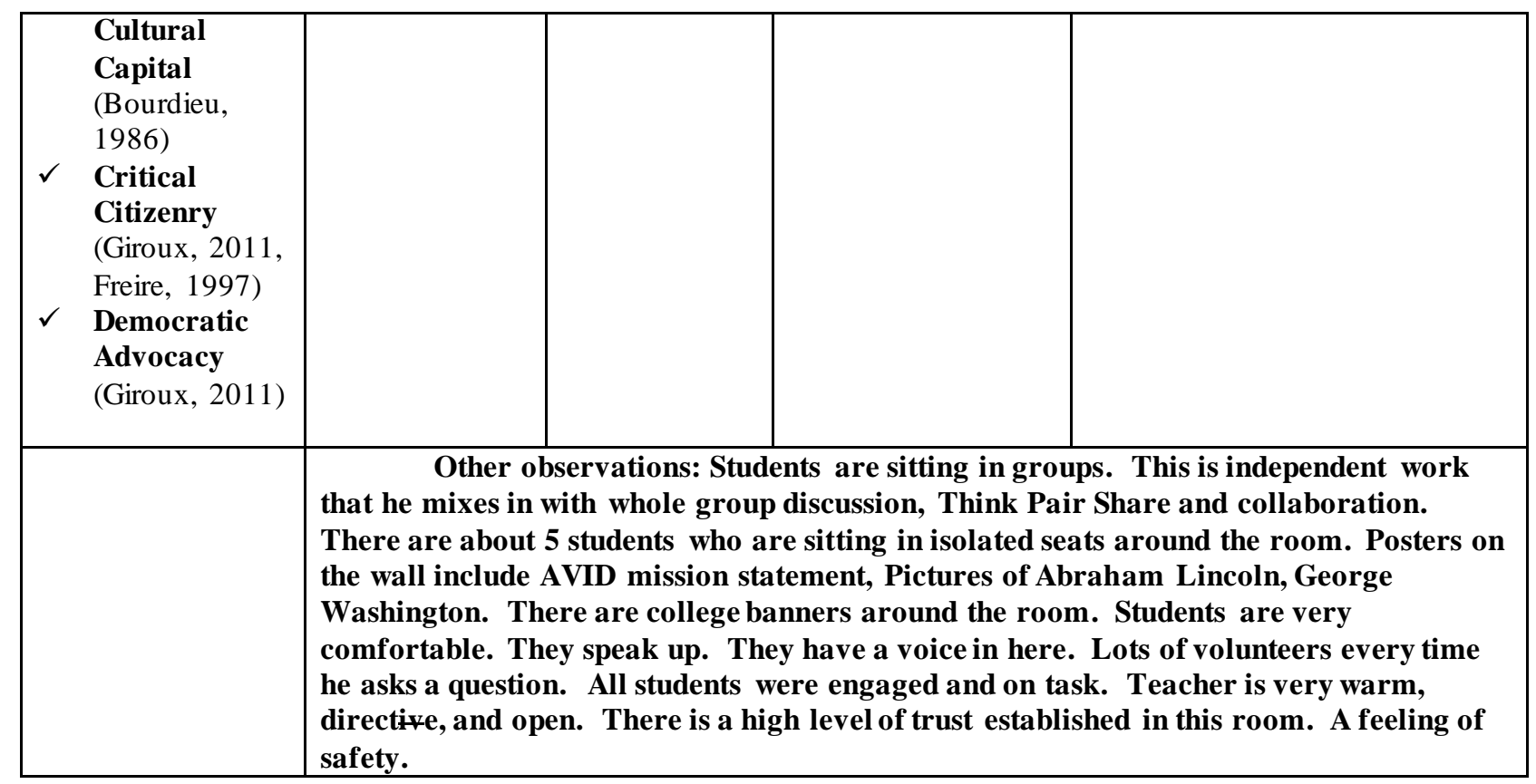


EXAMINING TEACHERS' PARADIGM AND PEDAGOGY IN MANIFESTING CRITICAL HOPE IN LOW-INCOME MIDDLE SCHOOL

Appendix G

Participant 7: Interview and Observation Matrix

\begin{tabular}{|c|c|c|}
\hline $\begin{array}{l}\text { Participant } \\
\text { P.7 5/29/19 }\end{array}$ & Questions & Comments \\
\hline Teacher & $\begin{array}{l}\text { Introduction: Thank you for } \\
\text { a greeing to participate in my study on } \\
\text { Examining Teachers' Paradigm and Pedagogy } \\
\text { in Manifesting Critical Hope in Low-Income } \\
\text { Middle School Classrooms: A Narrative } \\
\text { Inquiry } \\
\text { This interview will last } \\
\text { approximately } 30 \text { to } 45 \text { minutes. I will be } \\
\text { a sking you to review the transcript of the } \\
\text { interview for accuracy. } \\
\text { 1. (PI's introduction to questions) } \\
\text { START WITH an OPEN-ENDED } \\
\text { QUESTION: JUST LIKE TELLING A } \\
\text { STORY: We already know through existing } \\
\text { research that there is an achievement gap } \\
\text { between low-income students and affluent } \\
\text { students. Tell me, how do you make your } \\
\text { low-income students a spire to be successful? } \\
\text { Follow-up questions to teacher's } \\
\text { 2. In your experience are you aware } \\
\text { storytelling: } \\
\text { of any factors that compliment students' desire } \\
\text { to be successful? }\end{array}$ & $\begin{array}{l}\text { 1. I think by trying to get, by making them } \\
\text { see how I relate to them, by making them see how I } \\
\text { came from a similar background and making them } \\
\text { see, for them to feel successful to see how } \\
\text { I grew up and my parents grew up. If they } \\
\text { were to see where I came from, the neighborhood, the } \\
\text { poverty, the discrimination to where I am now, they } \\
\text { can see that hope and inspiration for them to see that } \\
\text { you can be successful in an upbringing that is similar } \\
\text { to mine. } \\
\text { 2. What would inspire for them to be } \\
\text { successful is for an example. I tell the story about my } \\
\text { parents because I think they might be from similar } \\
\text { backgrounds as my parents from working long hours } \\
\text { or laborious jobs and seeing how my parents } \\
\text { struggled inspired me. Hopefully observing the way } \\
\text { their parents struggle would inspire them to want to } \\
\text { do better and in the long run to want to help out their } \\
\text { parents, but through good careers not something } \\
\text { laborious that is going to break their backs. } \\
\text { 3. I think if they see a lot of poverty around } \\
\text { them, or people who do not really move out of their } \\
\text { communities that they will feel stuck that they will } \\
\text { feel it is not likely that they will be able to make it out } \\
\text { of their communities as well. Parents say they need } \\
\text { to be a hard worker so they may feel they need to } \\
\text { have a laborious job, to be valued because people can } \\
\text { interpret hard work as physical work. }\end{array}$ \\
\hline
\end{tabular}




\section{EXAMINING TEACHERS' PARADIGM AND PEDAGOGY IN MANIFESTING CRITICAL HOPE IN LOW-INCOME MIDDLE SCHOOL}

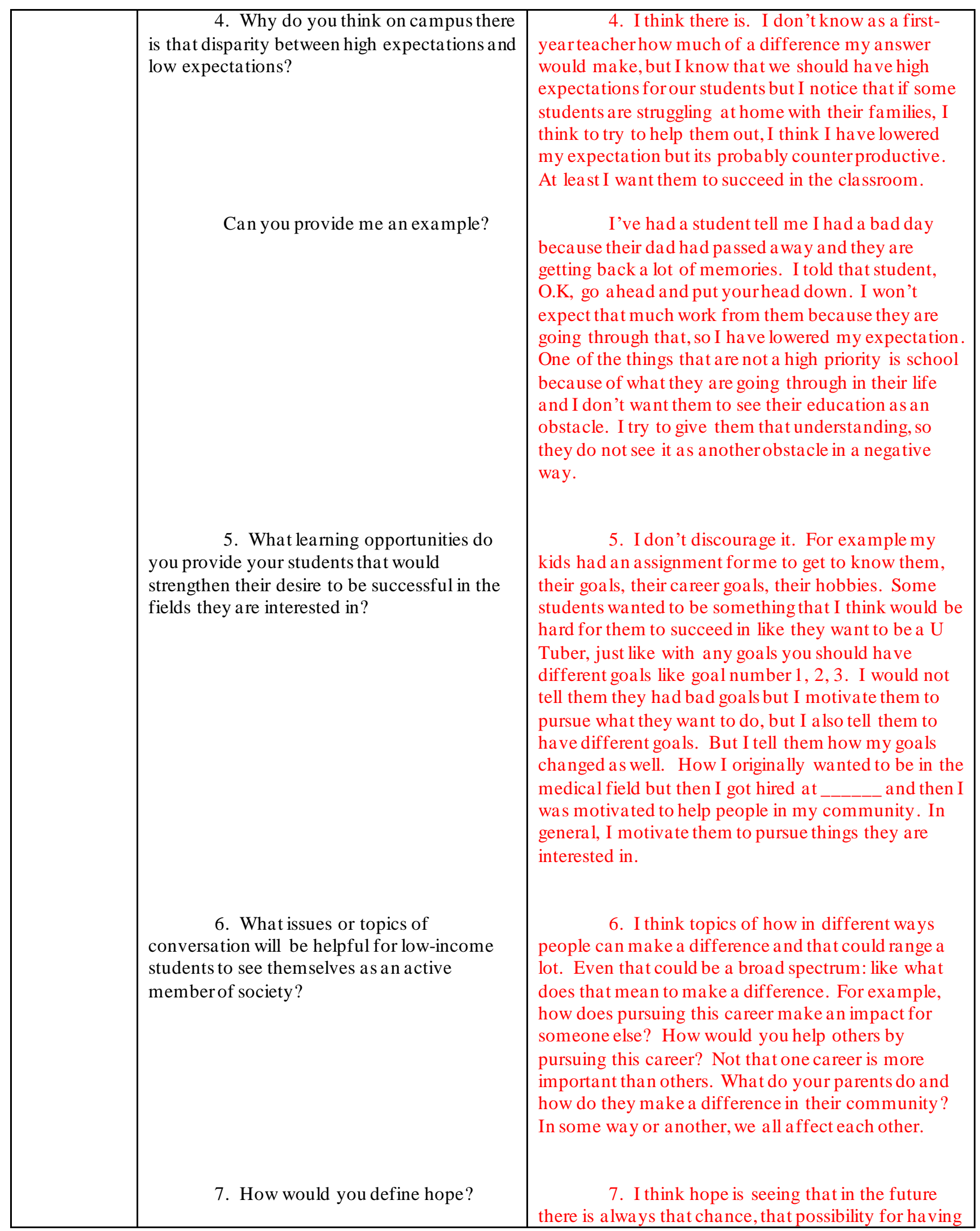




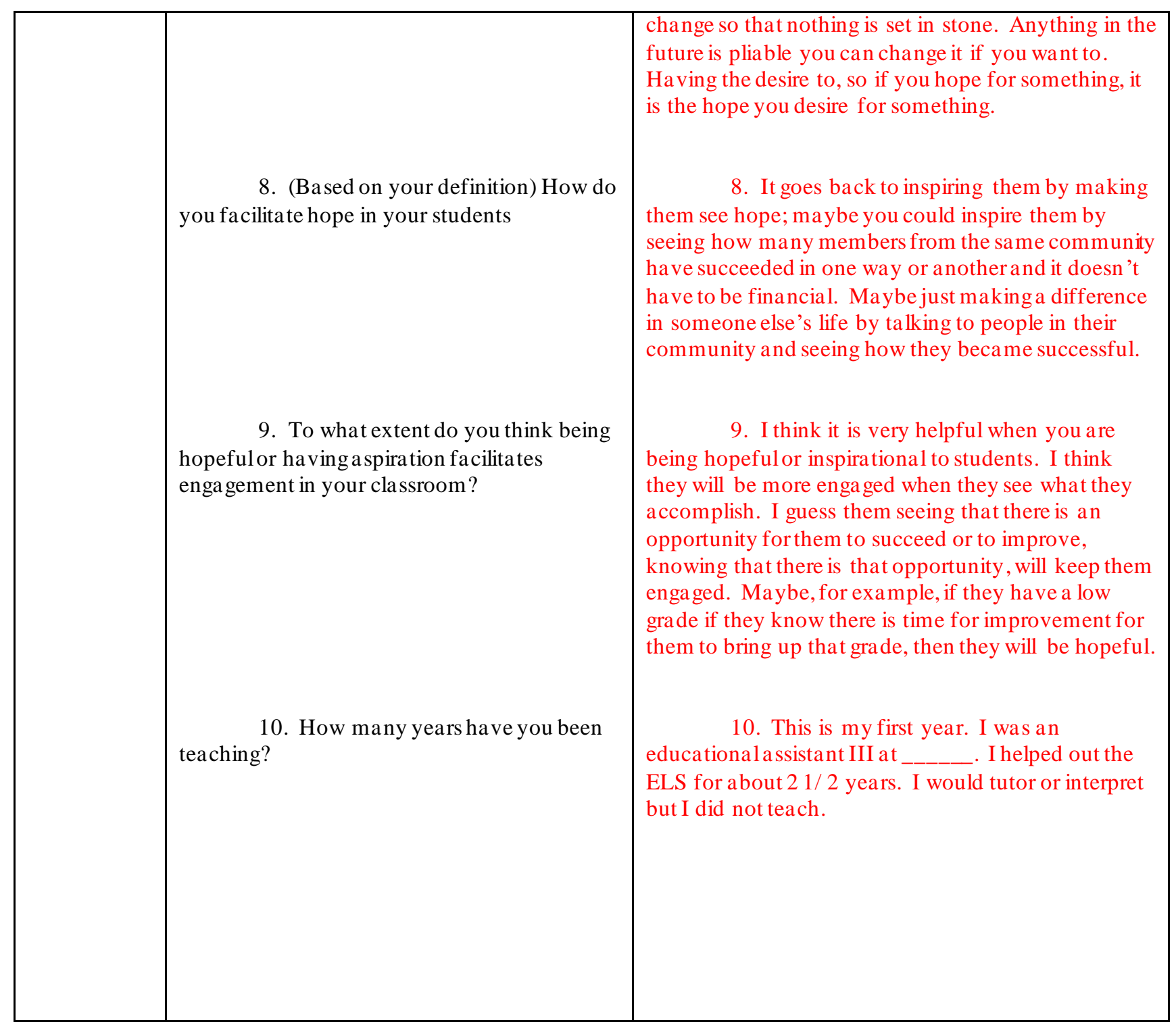




\section{Manifestations of Critical Hope Reflected in Teachers' Paradigm and Pedagogy}

\section{Observation Matrix}

(For observing elements of Critical Hope manifested in teachers' language and behavior of hope, teachers' instructional delivery of hope, and teachers' lesson planning of hope)

\section{The Teacher}

Time Observed: $\_$P-7
Observer:
F4
Date: 9:50 5/29/19

\begin{tabular}{|c|c|c|c|c|}
\hline $\begin{array}{l}\text { Manifestations of } \\
\text { Critical Hope } \\
\text { Elements in Teachers' } \\
\text { Paradigm \& } \\
\text { Pedagogy }\end{array}$ & $\begin{array}{l}\text { Manifestations } \\
\text { of Critical } \\
\text { Hope in: }\end{array}$ & $\begin{array}{l}\text { General } \\
\text { Indicators }\end{array}$ & Examples & Observed Evidence \\
\hline $\begin{array}{ll}\checkmark & \text { Goal Directed } \\
\text { Thinking } \\
\checkmark & \text { Navigating } \\
\text { Pathways } \\
\checkmark & \text { Willpower or } \\
\text { Agency } \\
\text { (Edwards, Rand, } \\
\text { Lopez, \& Snyder, } \\
\text { 2007) }\end{array}$ & $\begin{array}{l}\text { Teachers use } \\
\text { of vocabulary } \\
\text { expression } \\
\text { and written } \\
\text { direction }\end{array}$ & 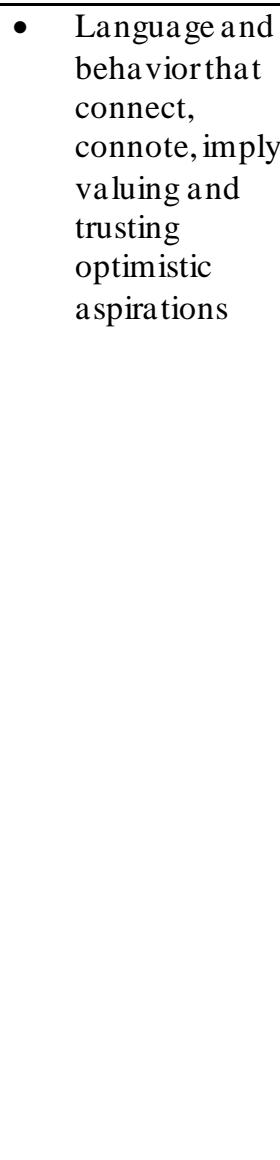 & $\begin{array}{cl}\circ & \begin{array}{l}\text { Positive } \\
\text { reinforcements }\end{array} \\
\circ & \begin{array}{l}\text { Positive } \\
\text { directives }\end{array} \\
\circ & \text { Praises } \\
\circ & \begin{array}{l}\text { Encouraging } \\
\text { statements }\end{array} \\
\circ & \text { Competence }\end{array}$ & $\begin{array}{l}\quad \text { I am going to } \\
\text { be recording for my } \\
\text { CAL TPA's today as } \\
\text { well. Hands a students } \\
\text { her phone to record in } \\
\text { the back. Today we are } \\
\text { going to be taking } 3 \\
\text { bullet point notes on the } \\
\text { Rock Cycle. If you } \\
\text { want more extra credit } \\
\text { take more than } 3 \text { notes } \\
\text { from the video. Please } \\
\text { get the lights. Thank } \\
\text { you. Teacheris } \\
\text { showing the video on } \\
\text { the rock cycle. Students } \\
\text { are taking notes. I can } \\
\text { tell they have done this } \\
\text { before. Teacher is } \\
\text { passing out supplies. } \\
\text { Each table gets a box. } \\
\text { Raise your hand and tell } \\
\text { me one fact you have } \\
\text { written down. Students' } \\
\text { gives answer and the } \\
\text { teacher repeats it. } \\
\text { Good. Asks for another } \\
\text { volunteer, teacher } \\
\text { repeats the student's }\end{array}$ \\
\hline
\end{tabular}




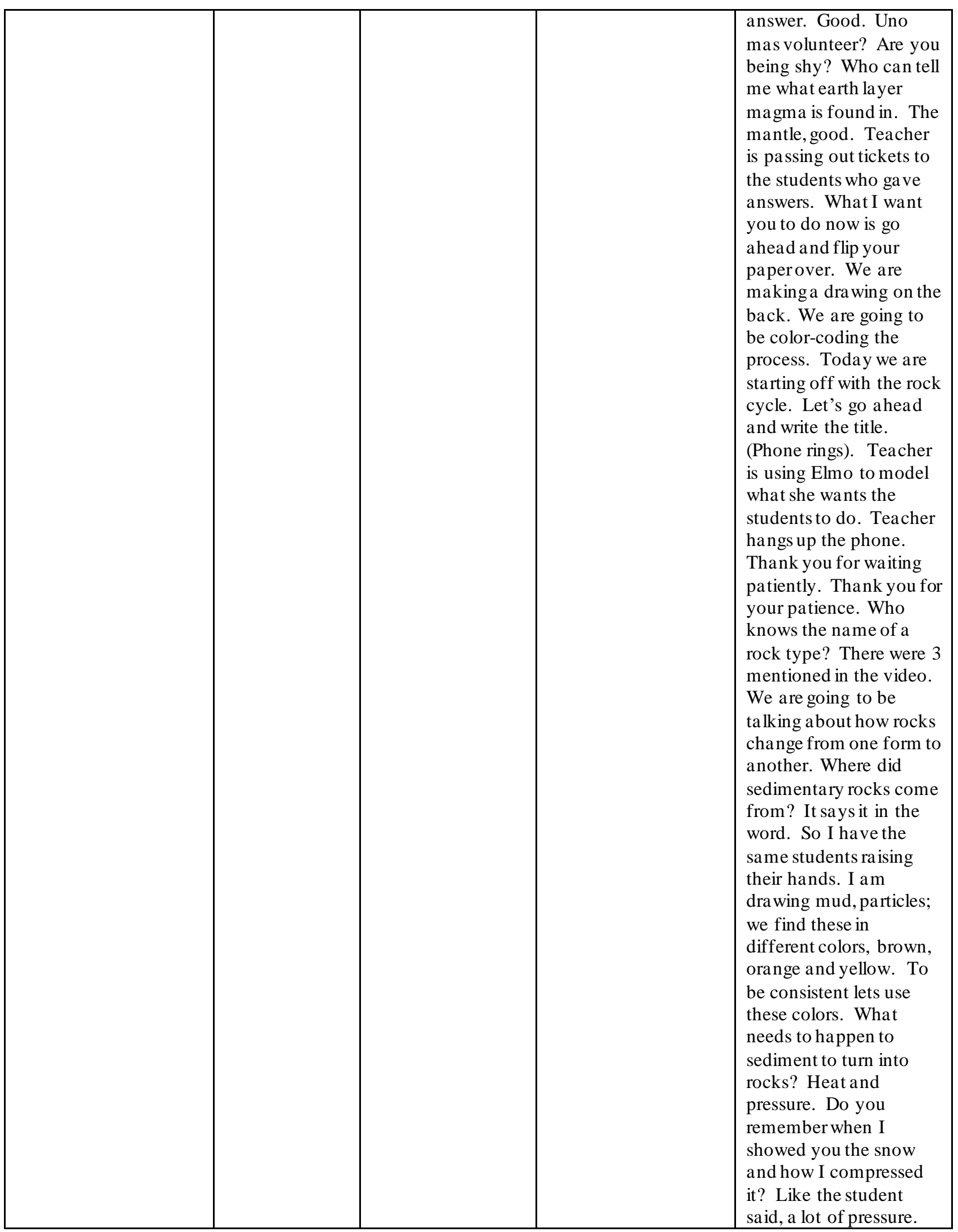




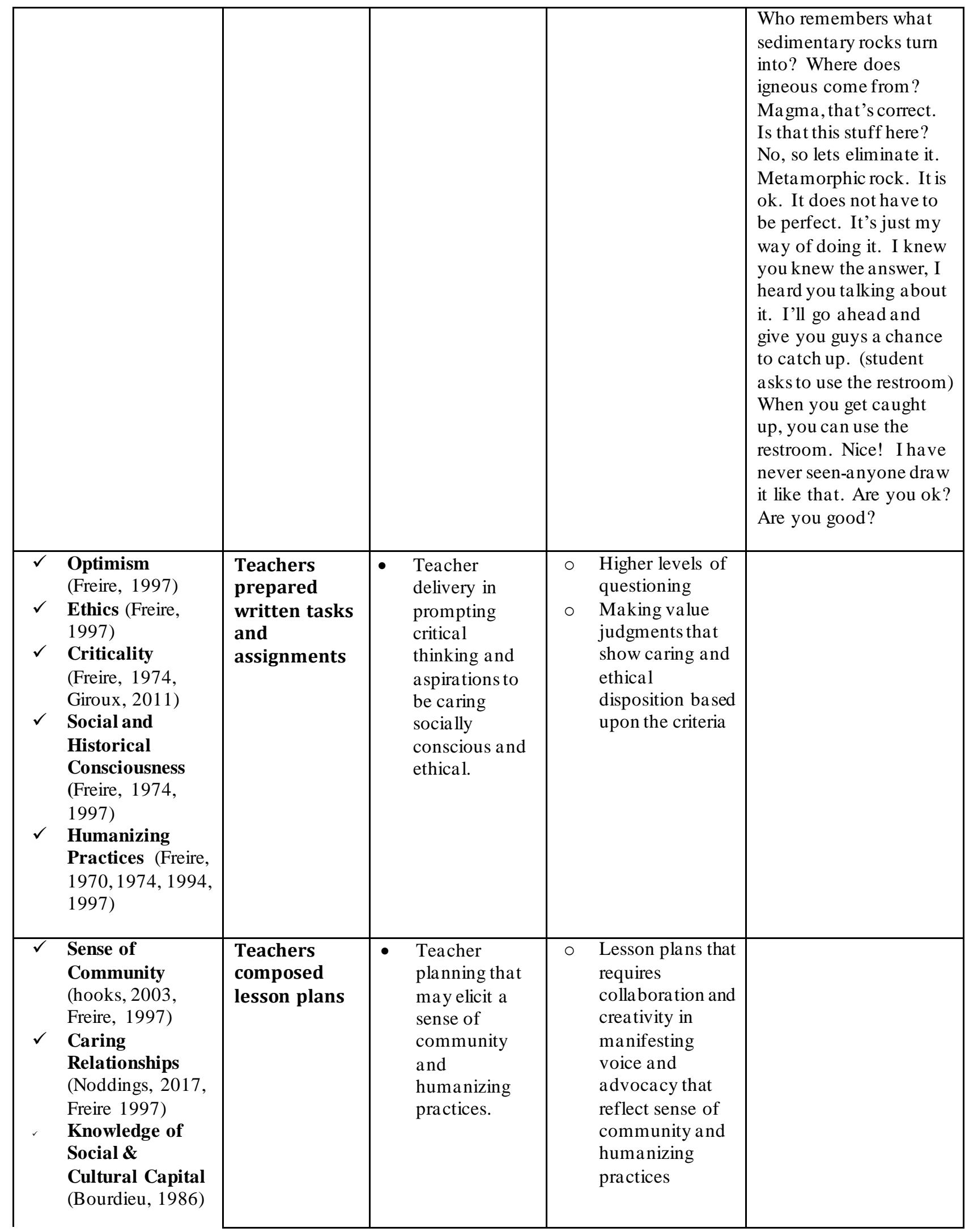


EXAMINING TEACHERS' PARADIGM AND PEDAGOGY IN MANIFESTING CRITICAL

HOPE IN LOW-INCOME MIDDLE SCHOOL

\begin{tabular}{|l|l|l|l|}
\hline$\checkmark$ & $\begin{array}{l}\text { Critical } \\
\text { Citizenry } \\
\text { (Giroux, 2011, }\end{array}$ \\
$\checkmark$ & $\begin{array}{l}\text { Freire, 1997) } \\
\text { Democratic } \\
\text { Advocacy } \\
\text { (Giroux, 2011) }\end{array}$ & & \\
\hline & \multicolumn{2}{|c|}{ Other observations: Room is very calm. Students are on task. Teacher has } \\
& $\begin{array}{l}\text { established relationships with the students. They respect the teacher and the teacher } \\
\text { respects them. }\end{array}$ \\
\hline
\end{tabular}


EXAMINING TEACHERS' PARADIGM AND PEDAGOGY IN MANIFESTING CRITICAL HOPE IN LOW-INCOME MIDDLE SCHOOL

\section{Appendix $\mathrm{H}$}

\section{Participant 8: Interview and Observation}

\begin{tabular}{|c|c|c|}
\hline $\begin{array}{l}\text { Participant } \\
\text { P.8 5/29/19 }\end{array}$ & Questions & Comments 8th grade math \\
\hline Teacher & $\begin{array}{l}\text { Introduction: Thank you for } \\
\text { agreeing to participate in my study on } \\
\text { Examining Teachers' Paradigm and Pedagogy } \\
\text { in Manifesting Critical Hope in Low-Income } \\
\text { Middle School Cla ssrooms: A Narrative } \\
\text { Inquiry } \\
\text { This interview will last } \\
\text { approximately } 30 \text { to } 45 \text { minutes. I will be } \\
\text { a sking you to review the transcript of the } \\
\text { interview for accuracy. } \\
\text { 1. (PI's introduction to questions) } \\
\text { STAllow-up questions to teacher's } \\
\text { of any factors that compliment students' desire } \\
\text { to be successful? } \\
\text { QUESTION: JUST LIKE TELLING A } \\
\text { STORY: We a lready know through existing } \\
\text { research that there is an achievement gap } \\
\text { between low-income students and affluent } \\
\text { students. Tell me, how do you make your } \\
\text { low-income students a spire to be successful? }\end{array}$ & $\begin{array}{l}\text { 1. That is a deep question let me think ... } \\
\text { So I believe that if kids see that they can be } \\
\text { successful then they are more likely to try to be } \\
\text { successful. First thing I do is I try to build a } \\
\text { community with my kids so they can see that I am } \\
\text { learning. Then I try to build that trust, so they can } \\
\text { learn from me. So I try to start with what they are } \\
\text { doing well and push them a little further. Instead of } \\
\text { starting with what they are doing wrong. I try to } \\
\text { focus on what they are doing right. Once they } \\
\text { experience some success it is contagious and they } \\
\text { want to try harder. I try to encourage them. I don't } \\
\text { want them to look at their present circumstances. I } \\
\text { came where you came from, I don't want you to say I } \\
\text { did not learn this because I came from King, I want } \\
\text { them to learn this well, they don't have to go to a } \\
\text { private school in a rich area; they can be successful } \\
\text { where they are. I hold them accountable and I hold } \\
\text { my self-accountable. Without that accountability } \\
\text { they will never experience that success. Then I tell } \\
\text { them to trust me and then I say try, if you try and you } \\
\text { trust me they will experience success. Do not give } \\
\text { me } 99 \text { percent give me } 100 \text { percent. If I give them } \\
\text { homework I want them to do their best. Try your } \\
\text { best, look back on what you have done and try to } \\
\text { mirror it. DO it for one week try for one week and } \\
\text { tell me how you feel; then I ask them what changed? } \\
\text { I have never seen one student who has tried for one } \\
\text { week and have never come back and said they did not } \\
\text { feel good. }\end{array}$ \\
\hline
\end{tabular}




\section{EXAMINING TEACHERS' PARADIGM AND PEDAGOGY IN MANIFESTING CRITICAL HOPE IN LOW-INCOME MIDDLE SCHOOL}

\begin{tabular}{|c|c|}
\hline$x^{2}$ & $\begin{array}{l}\text { to want to do more. } \\
\text { 3. I mean there are a lot of things. I'm } \\
\text { going to talk bout something I don't have control over } \\
\text { like their family situation, they do not have anybody } \\
\text { they look up to that want them to be at the level we } \\
\text { are at. So we are their examples. A lot of them say } \\
\text { my dad has never been to school and he does not have } \\
\text { a good job. They do not have any real example; they } \\
\text { do not have anyone to help them at home. To tutor or } \\
\text { to help them these kids do not have access to that. A } \\
\text { lot of them it's not an option to stay after school } \\
\text { because of safety. There are roadblocks and some of } \\
\text { them have attitude. A student today had a cut and } \\
\text { wanted a band aid and she was telling me that she } \\
\text { was cleaning the floor and a plate broke and cut her } \\
\text { hand. A lot of these kids are dong adult things and } \\
\text { taking care of their homes } \\
\text { Some things we have controlover is adult } \\
\text { attitude. Adults can feel that they have no bearing } \\
\text { when they are not doing well but they are doing well } \\
\text { they take responsibility that they are doing well. If I } \\
\text { am focused and concentrated in teaching but not } \\
\text { focused on if they are learning, then that can hinder } \\
\text { their success. Also if I have low expectations. Just } \\
\text { because these kids are coming from a different place } \\
\text { does not mean they cannot learn at the same level as I } \\
\text { do. Does not mean they cannot reach high } \\
\text { expectations. My expectations can hinder them. } \\
\text { Their well being, If they are hungry I can choose to } \\
\text { provide something for them. Well I bring all these } \\
\text { oranges I don't bring them just for myself. I know I } \\
\text { have student who haven't eaten. The granola bars, } \\
\text { the students have been eating these bars. Because } \\
\text { they don't have breakfast making sure they are eating } \\
\text { well. Some of them didn't even sleep. Sometimes } \\
\text { they tell me 'I have a headache so I let them put it } \\
\text { down for } 5 \text { min. You know what the student is going } \\
\text { to do or me, he will work for me because they know } \\
\text { that I care more about them than just math. That girl, } \\
\text { her mom has cancer, she talks to me. I know you are } \\
\text { going through these things, I tell her you are working } \\
\text { so hard and you will be able to take care of her. }\end{array}$ \\
\hline $\begin{array}{l}\text { 4. Why do you think on campus } \\
\text { there is that disparity between high } \\
\text { expectations and low expectations? }\end{array}$ & $\begin{array}{l}\text { 4. Amongst teachers, there is not one } \\
\text { answer to that; I think it has to do with more of you as } \\
\text { a person. What is your goal, what is your motive for } \\
\text { teaching; your why? If my why is to see my students } \\
\text { succeed then I know that I will have high } \\
\text { expectations. If they are not learning the } \\
\text { responsibility involves me. What drives us? Is it a } \\
\text { paycheck; is it that I want something to do? }\end{array}$ \\
\hline
\end{tabular}




\section{EXAMINING TEACHERS'PARADIGM AND PEDAGOGY IN MANIFESTING CRITICAL HOPE IN LOW-INCOME MIDDLE SCHOOL}

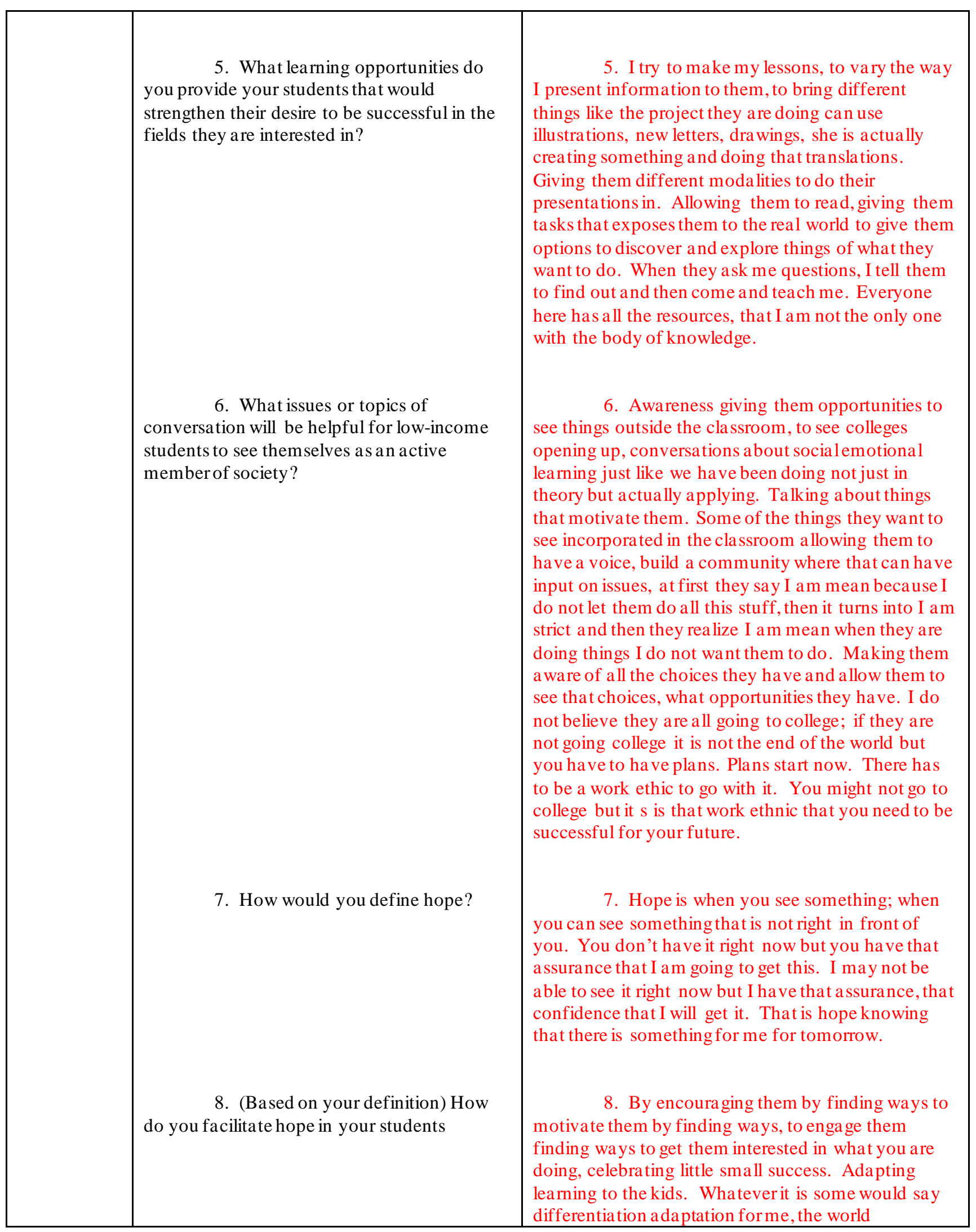




\section{EXAMINING TEACHERS' PARADIGM AND PEDAGOGY IN MANIFESTING CRITICAL HOPE IN LOW-INCOME MIDDLE SCHOOL}

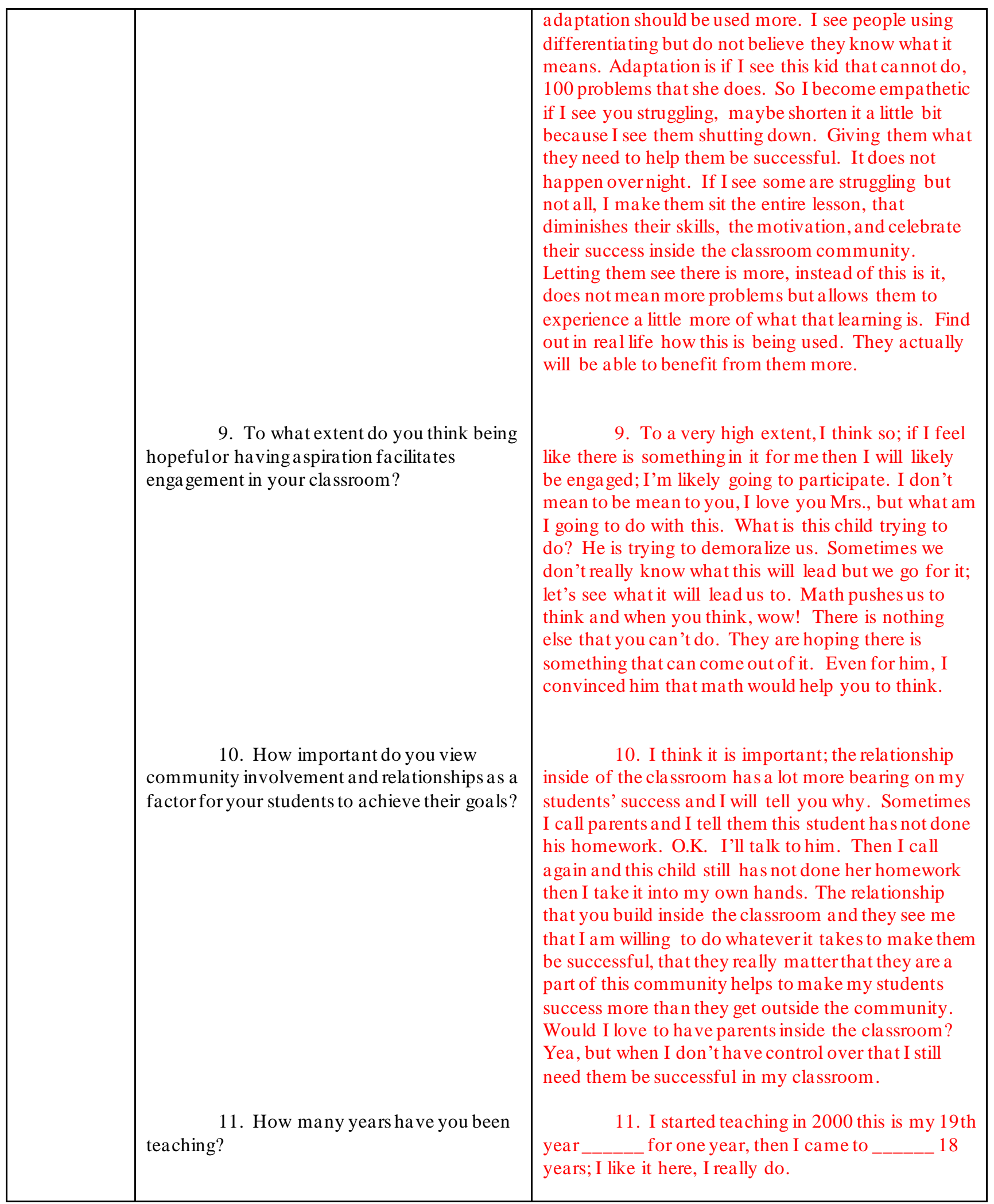


EXAMINING TEACHERS' PARADIGM AND PEDAGOGY IN MANIFESTING CRITICAL HOPE IN LOW-INCOME MIDDLE SCHOOL

\section{Manifestations of Critical Hope Reflected in Teachers' Paradigm and Pedagogy}

\section{Observation Matrix}

(For observing elements of Critical Hope manifested in teachers' language and behavior of hope, teachers' instructional delivery of hope, and teachers' lesson planning of hope)

\section{The Teacher \\ Time Observed:}
Observer:
F2
Date: 1:38 5/29/18

\begin{tabular}{|c|c|c|c|c|}
\hline $\begin{array}{l}\text { Manifestations of } \\
\text { Critical Hope } \\
\text { Elements in } \\
\text { Teachers' } \\
\text { Paradigm \& } \\
\text { Pedagogy }\end{array}$ & $\begin{array}{l}\text { Manifestations } \\
\text { of Critical } \\
\text { Hope in: }\end{array}$ & $\begin{array}{l}\text { General } \\
\text { Indicators }\end{array}$ & Examples & Observed Evidence \\
\hline $\begin{array}{ll}\checkmark & \text { Goal Directed } \\
& \text { Thinking } \\
\checkmark & \text { Navigating } \\
& \text { Pathways } \\
\checkmark & \text { Willpower or } \\
& \text { Agency } \\
& \text { (Edwards, } \\
& \text { Rand,Lopez, } \\
& \& \text { Snyder, } \\
& 2007 \text { ) }\end{array}$ & $\begin{array}{l}\text { Teachers use } \\
\text { of vocabulary } \\
\text { expression } \\
\text { and written } \\
\text { direction }\end{array}$ & $\begin{array}{l}\text { - Language } \\
\text { and beha vior } \\
\text { that connect, } \\
\text { connote, } \\
\text { imply } \\
\text { valuing and } \\
\text { trusting } \\
\text { optimistic } \\
\text { aspirations }\end{array}$ & $\begin{array}{ll}\circ & \begin{array}{l}\text { Positive } \\
\text { reinforcements }\end{array} \\
\circ & \text { Positive } \\
& \text { directives } \\
\circ & \text { Praises } \\
\circ & \text { Encouraging } \\
& \text { statements } \\
\circ & \text { Competence }\end{array}$ & $\begin{array}{l}\text {-What type of city are } \\
\text { you trying to build? Research it } \\
\text { and then, go back to your city. } \\
\text { Students are making projects. } \\
\text { They have iPads' and are } \\
\text { working in small flexible } \\
\text { groupings. Teacher is going } \\
\text { from person to person. You do } \\
\text { not need me to tell you. You } \\
\text { need to read this and it will tell } \\
\text { you everything. Where is your } \\
\text { ruler? Make sure you have it. } \\
\text { There is a ruler in the bag if you } \\
\text { need it. What should you be } \\
\text { doing right now? Let me see. } \\
\text { Make sure it does not get stuck } \\
\text { there. You are going to have to } \\
\text { really do this, do the homework } \\
\text { first, and follow the directions. } \\
\text { Student asks what is } \\
\text { transversal? Yes, do you know } \\
\text { what transversalis? Yes, let's } \\
\text { ask your peer, all right, a sks } \\
\text { whole class. What is a } \\
\text { transversal? Someone is a sking } \\
\text { me what a transversalis. I see } \\
\text { one hand,two, three. Only } \\
\text { three people can tell me what a }\end{array}$ \\
\hline
\end{tabular}




\begin{tabular}{|c|c|c|c|c|}
\hline & & & & $\begin{array}{l}\text { transversalis? A transversal } \\
\text { line, so what is a transversal } \\
\text { line? Can it also be like this? It } \\
\text { is reflecting? Is that true? } \\
\text { Starts to draw on the } \\
\text { whiteboard. It is a line that } \\
\text { intersects two lines, they do not } \\
\text { have to be parallel lines because } \\
\text { it cuts two or more, but when it } \\
\text { cuts through the parallel lines } \\
\text { we have these special } \\
\text { relationships. Does that make } \\
\text { sense? Goes back to the } \\
\text { original student who had the } \\
\text { question. So do you know what } \\
\text { transversalline is? They have } \\
\text { the same what? Students are } \\
\text { corralling "rate of change" they } \\
\text { have the same what? "Rate of } \\
\text { change." It is not that they look } \\
\text { parallel you have to prove they } \\
\text { are parallel it is because they } \\
\text { have the same rate of change or } \\
\text { the same slope. Sit like a } \\
\text { scholar. Students are back on } \\
\text { their assignments. }\end{array}$ \\
\hline 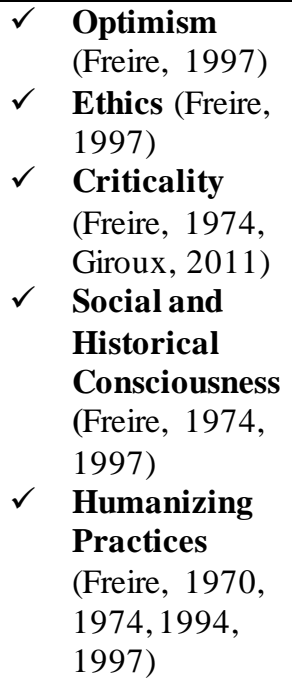 & $\begin{array}{l}\text { Teachers } \\
\text { prepared } \\
\text { written tasks } \\
\text { and } \\
\text { assignments }\end{array}$ & $\begin{array}{l}\text { Teacher } \\
\text { delivery in } \\
\text { prompting } \\
\text { critical } \\
\text { thinking and } \\
\text { a spirations } \\
\text { to be caring } \\
\text { socially } \\
\text { conscious } \\
\text { and ethical. }\end{array}$ & $\begin{array}{ll}\circ & \text { Higher levels } \\
\text { of questioning } \\
\text { Making value } \\
\text { judgments that } \\
\text { show caring } \\
\text { and ethical } \\
\text { disposition } \\
\text { based upon the } \\
\text { criteria }\end{array}$ & \\
\hline $\begin{array}{ll}\checkmark & \begin{array}{l}\text { Sense of } \\
\text { Community }\end{array} \\
& \text { (hooks, 2003, } \\
& \text { Freire, 1997) } \\
\checkmark & \text { Caring } \\
\text { Relationships } \\
\text { (Noddings, } \\
\text { 2017, Freire } \\
\text { 1997) }\end{array}$ & $\begin{array}{l}\text { Teachers } \\
\text { composed } \\
\text { lesson plans }\end{array}$ & $\begin{array}{l}\text { - Teacher } \\
\text { planning that } \\
\text { may elicit a } \\
\text { sense of } \\
\text { community } \\
\text { and } \\
\text { humanizing } \\
\text { practices. }\end{array}$ & $\begin{array}{l}\text { Lesson plans } \\
\text { that requires } \\
\text { collaboration } \\
\text { and creativity } \\
\text { in manifesting } \\
\text { voice and } \\
\text { advocacy that } \\
\text { reflect sense of } \\
\text { community }\end{array}$ & \\
\hline
\end{tabular}




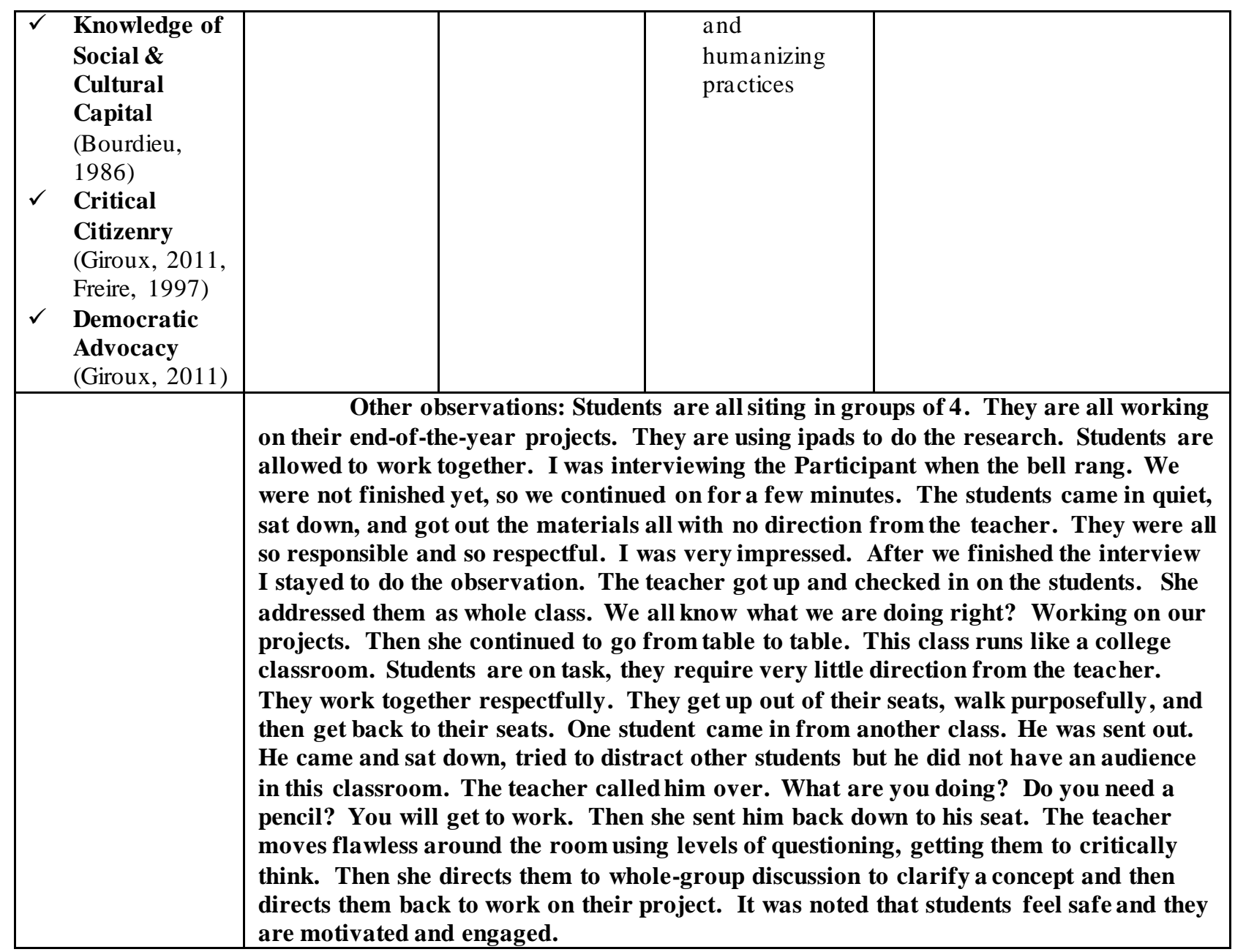


EXAMINING TEACHERS' PARADIGM AND PEDAGOGY IN MANIFESTING CRITICAL
HOPE IN LOW-INCOME MIDDLE SCHOOL

Appendix I

Participant 9: Interview and Observation

\begin{tabular}{|c|c|c|}
\hline $\begin{array}{l}\text { Participant } \\
\text { P.9 5/29/19 }\end{array}$ & Questions & Comments \\
\hline \multirow[t]{2}{*}{ Teacher } & $\begin{array}{l}\text { Introduction: Thank you for } \\
\text { agreeing to participate in my study on } \\
\text { Examining Teachers' Paradigm and Peda gogy } \\
\text { in Manifesting Critical Hope in Low-Income } \\
\text { Middle School Classrooms: A Narrative } \\
\text { Inquiry } \\
\text { This interview will last } \\
\text { approximately } 30 \text { to } 45 \text { minutes. I will be } \\
\text { asking you to review the transcript of the } \\
\text { interview for accuracy. } \\
\text { 1. (PI's introduction to questions) } \\
\text { START WITH an OPEN-ENDED } \\
\text { QUESTION: JUST LIKE TELLING A } \\
\text { STORY: We already know through existing } \\
\text { research that there is an achievement gap } \\
\text { between low-income students and affluent } \\
\text { students. Tell me, how do you make your } \\
\text { low-income students a spire to be successful? } \\
\text { Follow-up questions to teacher's } \\
\text { storytelling: } \\
\text { 2. In your experience are you aware } \\
\text { of any factors that compliment students' } \\
\text { desire to be successful? }\end{array}$ & $\begin{array}{l}\text { 1. Things I do in the classroom: I explain to } \\
\text { them that what we are doing in the classroom makes } \\
\text { them ready for jobs and prepares them for college, } \\
\text { which can help them with the income. I try to make a } \\
\text { connection between what we are doing in here and } \\
\text { what they want to do in the future. School gives you } \\
\text { choices. The more you do in school, the more } \\
\text { educated you are, the more choices are opened up for } \\
\text { you. Today, I pulled in several students to talk about } \\
\text { their potentialand their leadership skills, and to } \\
\text { encourage them to use them in a productive way. I } \\
\text { told them that they had gifts and they should use those } \\
\text { gifts, and that I did not see them using it for the best } \\
\text { right now. } \\
\qquad 2 \text {. I try to find their strengths and skills and } \\
\text { give them positive feedback on those things and I try } \\
\text { to be open and positive about things that happened for } \\
\text { me and how I tried to make it better, and so can they. } \\
\text { In the beginning of the yearI mentioned how I had a } \\
\text { hard time in math in middle school and I talked about } \\
\text { things that I learned that helped me to overcome that. } \\
\text { Just like the notebooks, trying to keep them organized } \\
\text { and successful in all their classes. My desire is for } \\
\text { them to be successful. I try to be open about how } \\
\text { things that may take practice now can be developed } \\
\text { into a skill and how you can use that to over come } \\
\text { them. }\end{array}$ \\
\hline & $\begin{array}{l}\text { 3. Are you aware of any factors that } \\
\text { would hinder these aspirations? }\end{array}$ & $\begin{array}{l}\text { 3. In the community we live in there are a } \\
\text { lot of things. I had a really gifted student that was } \\
\text { missing every day. I know she is gifted and can do } \\
\text { things really well. I found out that she has family }\end{array}$ \\
\hline
\end{tabular}




\section{EXAMINING TEACHERS' PARADIGM AND PEDAGOGY IN MANIFESTING CRITICAL HOPE IN LOW-INCOME MIDDLE SCHOOL}

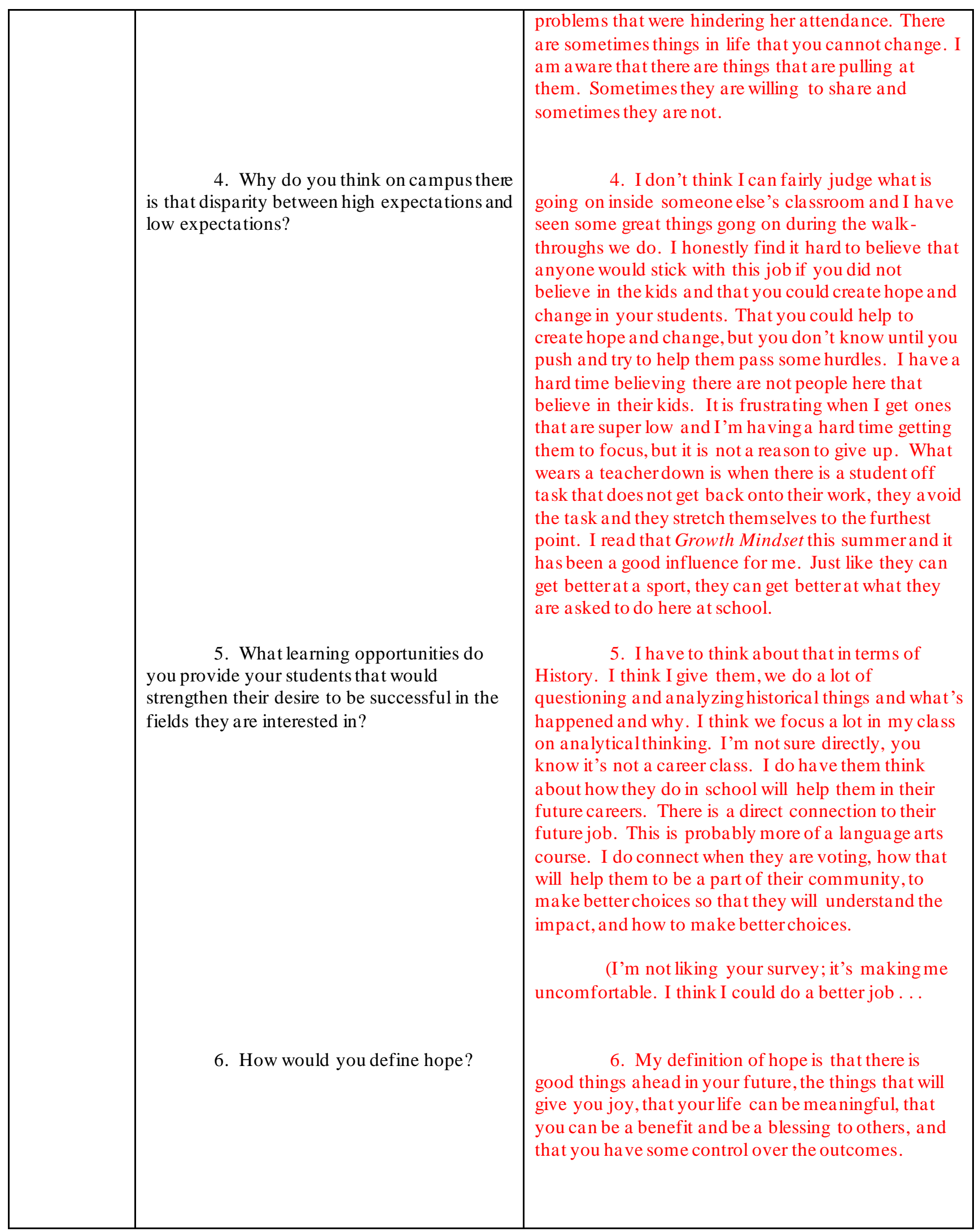




\section{EXAMINING TEACHERS' PARADIGM AND PEDAGOGY IN MANIFESTING CRITICAL HOPE IN LOW-INCOME MIDDLE SCHOOL}

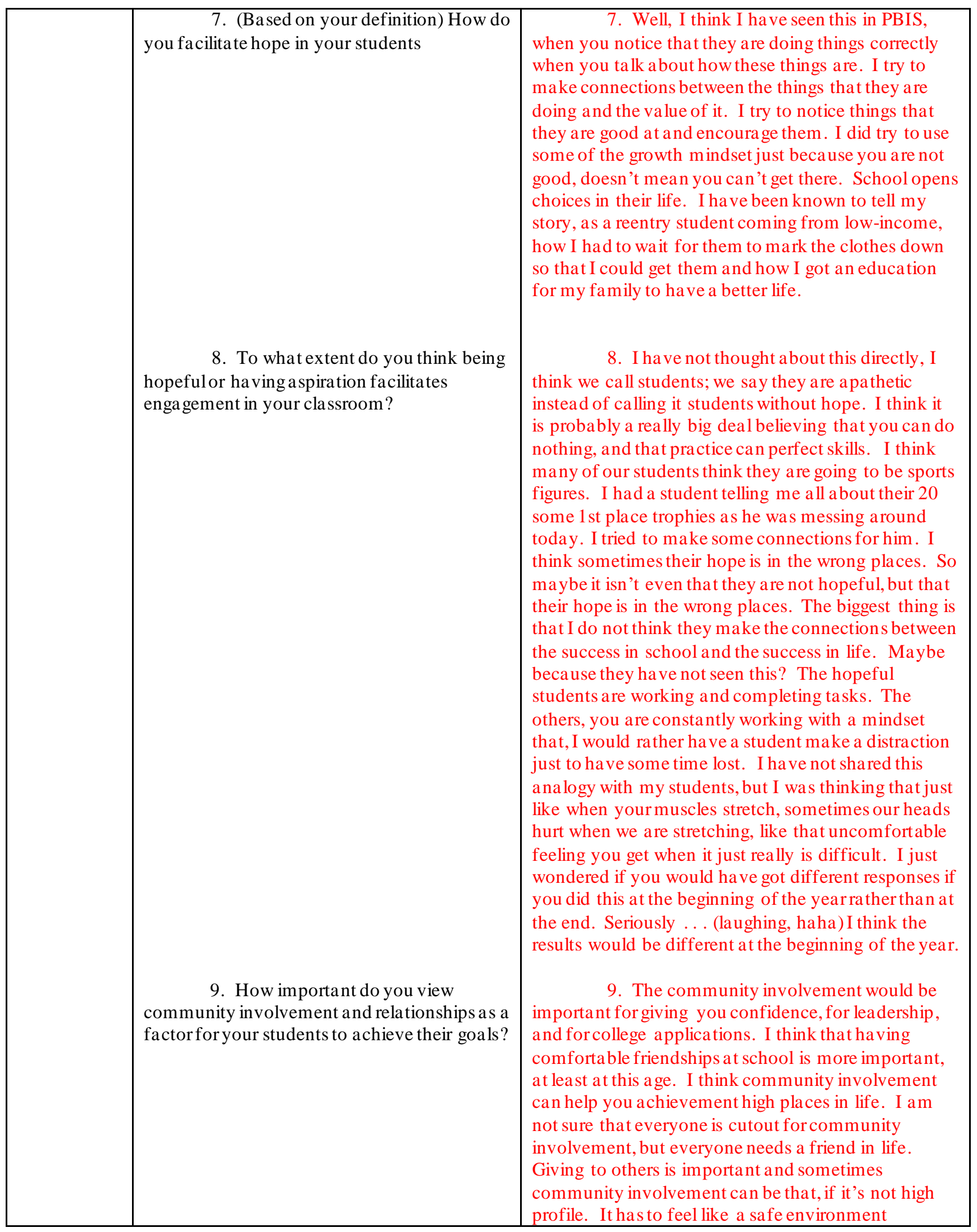


EXAMINING TEACHERS' PARADIGM AND PEDAGOGY IN MANIFESTING CRITICAL HOPE IN LOW-INCOME MIDDLE SCHOOL

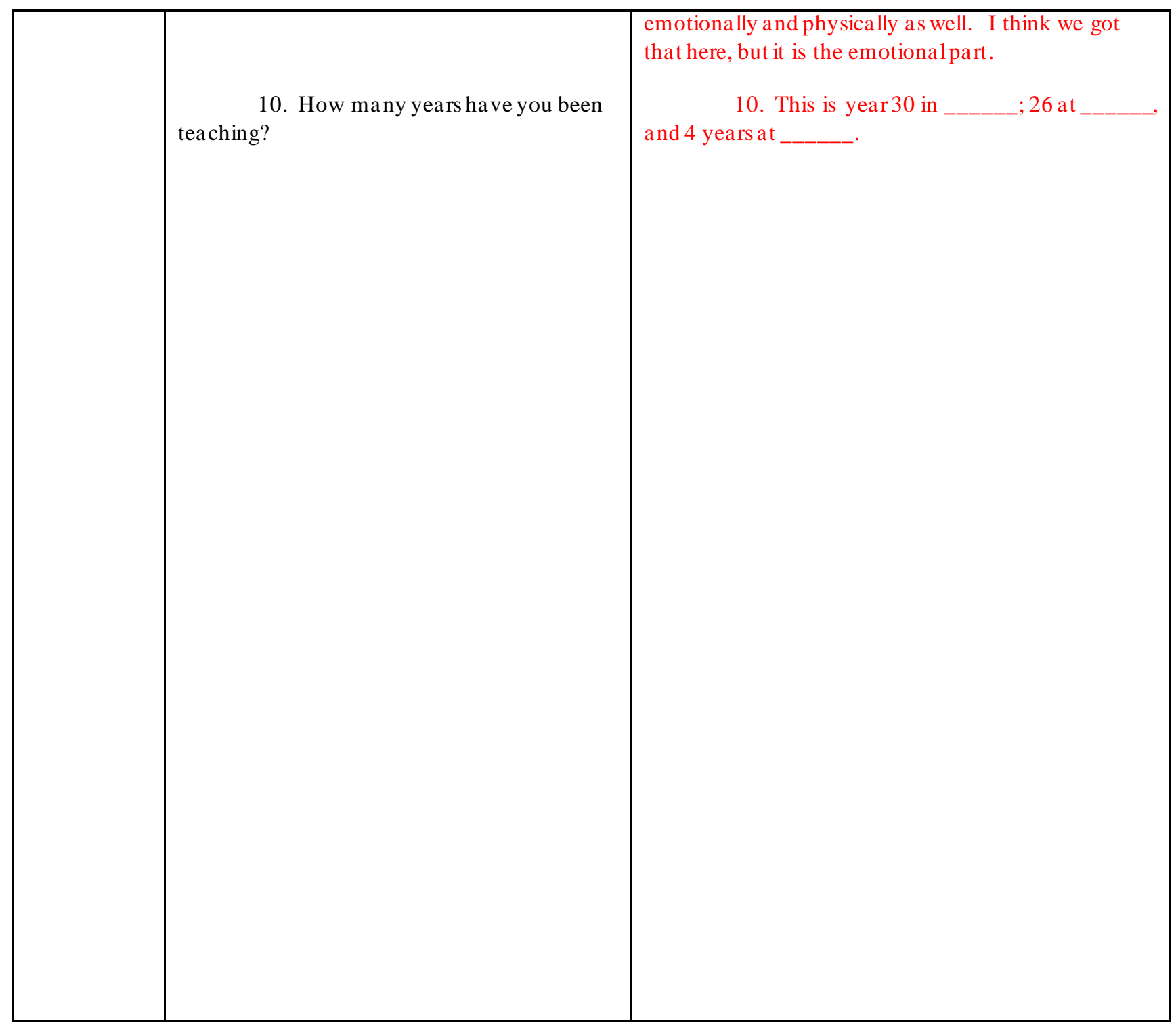

Observation: Participant 9 declined the observation. 


\section{EXAMINING TEACHERS' PARADIGM AND PEDAGOGY IN MANIFESTING CRITICAL HOPE IN LOW-INCOME MIDDLE SCHOOL}

\section{Appendix J}

\section{Participant 10: Interview and Observation}

\begin{tabular}{|c|c|c|}
\hline $\begin{array}{l}\text { Participant } \\
\text { P.2 5/24/19 }\end{array}$ & Questions & Comments \\
\hline Teacher & $\begin{array}{l}\text { Introduction: Thank you for agreeing } \\
\text { to participate in my study on Examining } \\
\text { Teachers' Paradigm and Pedagogy in } \\
\text { Manifesting Critical Hope in Low-Income } \\
\text { Middle School Classrooms: A Narrative Inquiry } \\
\text { This interview will last approximately } \\
30 \text { to } 45 \text { minutes. I will be asking you to review } \\
\text { the transcript of the interview for accuracy. } \\
\qquad \text { 1. (PI's introduction to questions) } \\
\text { START WITH an OPEN-ENDED QUESTION: } \\
\text { JUST LIKE TELLING A STORY: We already } \\
\text { know through existing research that there is an } \\
\text { achievement gap between low-income students } \\
\text { and affluent students. Tell me, how do you } \\
\text { make your low-income students aspire to be } \\
\text { successful? } \\
\text { Follow-up question: Can you provide an } \\
\text { example? } \\
\text { Follow-up questions to teacher's } \\
\text { 2. In your experience are you aware } \\
\text { storytelling: } \\
\text { of any factors that compliment students' desire } \\
\text { to be successful? }\end{array}$ & $\begin{array}{l}\text { 2. From what I have experienced here, I think } \\
\text { family dynamic plays a big factor in that students have a more } \\
\text { stable environment at home are in regular attendance, they } \\
\text { work hard, and have high expectations asset. My other } \\
\text { students who come from foster or have parents in jail, I feel } \\
\text { like I have to council them first before they even try. For } \\
\text { example I have a student whose dad is n jail and she says I'm } \\
\text { going to end up in jail any ways, why even try. I have to } \\
\text { counselor her to try. } \\
\qquad 3 \text {. Living with alternate family members, negativity } \\
\text { towards their progress is one thing that can really set them } \\
\text { back. I have a student I gave an award for most improved. } \\
\text { His parent called me and was upset with me for giving him } \\
\text { that award because they said that he did not even know his } \\
\text { ABC's; why did I give him an award. I let her vent and go off, } \\
\text { I like to meet students where they are at; from there we make } \\
\text { goals; Let's progress. She gave me an earful. I had never } \\
\text { been yelled at for giving a student an award. I noticed he } \\
\text { stopped trying because he must have got harped at home. It } \\
\text { was interesting because I had an IEP three weeks later and at } \\
\text { the IEP she did not question me. He has done so much better } \\
\text { this year. This is a kid who would not even write a letter A on } \\
\text { his paper and now he is a writing sentence. }\end{array}$ \\
\hline & & 4. I think a lot of times people don't want to poke \\
\hline
\end{tabular}




\section{EXAMINING TEACHERS' PARADIGM AND PEDAGOGY IN MANIFESTING CRITICAL HOPE IN LOW-INCOME MIDDLE SCHOOL}

there is that disparity between high expectations and low expectations?

5. What learning opportunities do you provide your students that would strengthen their desire to be successful in the fields they are interested in?

6. What issues or topics of conversation will be helpful for low-income students to see themselves as an active member of society?

7. How would you define hope?

8. (Based on your definition) How do you facilitate hope in your students

9. To what extent do you think being hopeful or having aspiration facilitates engagement in your classroom?

10. How important do you view community involvement and relationships as a factor for your students to achieve their goals? the bear with those who have behavior issues, so they just let them fall to the wayside. There is really not a consistency with consequences and a lot of my parents do not back me up when it comes to academics. The parents that are more supportive; their children do a lot better. They get it at both ends. At here and at home.

5. The counselor has been great in involving us in things that are off campus , where students got to go and observe, a conference for girls to empower them and be confident and make better decisions. As far as in class, I try to give them the freedom of choice is some projects we do that way they can take control of their learning. We do a lot of hands on. I love hands on.

6. A lot of our kids, we talk about goal setting, their future careers, their strengths, what they are good at. We do touch on stuff about how we treat others through social media, being respectful. Being good humans in general. We read Wonder; being opened in general, greeting others, and approaching people who are different.

7. Believing no matter now what is going on around you, believing that you are capable of achieving your goals. Knowing that there is more even if stuff is going on.

8. We try to build a relationship with them, make things fun, encourage them to try new things, even if they fail its ok. Regardless of what is going on at home, we encourage them to keep going; that they can make a better life for themselves. It is about them and not their parents.

9. To a very high extent, I guess because I have a lens from being here this year and last year and I see how they treat others inside the classroom and outside the classroom, they are goal setting on their own now and telling us what they want to do. I had the same kids for two years. I came in midyear last and they were a nightmare to be honest, kids cussing at me, throwing things at me; it was horrible. Then summer came and they came in able to follow the routine and are a lot more kind with one another. Kids that snapped at each other are now kind to each other and helping to learn how to read.

My thing is they are kids; they start new everyday with me. It's funny, a student that tried to fight me last year says now I'm her favorite teacher. It took awhile for them to trust me, to accept me. They came in at the beginning of the year and they said you're not mad at me and I said no. The big thing was they said you're not coming back and then I did.

10. Community involvement when it is positive is very important. When you think about the community you live around, there is a lot of negativity. It is important to plug into the positives. You guys all mom us, it's a culture where 
EXAMINING TEACHERS' PARADIGM AND PEDAGOGY IN MANIFESTING CRITICAL HOPE IN LOW-INCOME MIDDLE SCHOOL

\begin{tabular}{|l|l|l|}
\hline 11. How many years have you been & $\begin{array}{l}\text { we try to create that it's comfortable in there; we might not } \\
\text { always agree; we keep it mellow. Today what you saw I could } \\
\text { not have done that last year! There was no way I could not sit } \\
\text { down last year. I could not have two students here and some } \\
\text { on computers. I can trust there are on the websites I have } \\
\text { asked them to be on. That is a huge shift. I can actually teach } \\
\text { this year. }\end{array}$ \\
$\begin{array}{l}\text { teaching? } \\
\text { 11. This is my first experience. I started here } \\
\text { Previously I was at year. This is my first full year. } \\
\text { for 4 years and an instructional paraprofessional for } 6 \text { years. }\end{array}$ \\
\end{tabular}


EXAMINING TEACHERS'PARADIGM AND PEDAGOGY IN MANIFESTING CRITICAL HOPE IN LOW-INCOME MIDDLE SCHOOL

\section{Manifestations of Critical Hope Reflected in Teachers' Paradigm and Pedagogy}

\section{Observation Matrix}

(For observing elements of Critical Hope manifested in teachers' language and behavior of hope, teachers' instructional delivery of hope, and teachers' lesson planning of hope)

\section{The Teacher \\ Time Observed: __P-10}

Observer: _ K5___ Date: 7:50am 5/30/19

\begin{tabular}{|c|c|c|c|c|}
\hline $\begin{array}{l}\text { Manifestations of } \\
\text { Critical Hope } \\
\text { Elements in } \\
\text { Teachers' Paradigm } \\
\text { \& Pedagogy }\end{array}$ & $\begin{array}{l}\text { Manifestations } \\
\text { of Critical } \\
\text { Hope in: }\end{array}$ & $\begin{array}{l}\text { General } \\
\text { Indicators }\end{array}$ & Examples & Observed Evidence \\
\hline $\begin{array}{cl}\checkmark & \text { Goal Directed } \\
& \text { Thinking } \\
\checkmark & \text { Navigating } \\
& \text { Pathways } \\
\checkmark & \text { Willpower or } \\
& \text { Agency } \\
& \text { (Edwards, Rand, } \\
& \text { Lopez, \& } \\
& \text { Snyder, 2007) }\end{array}$ & $\begin{array}{l}\text { Teachers use } \\
\text { of vocabulary } \\
\text { expression } \\
\text { and written } \\
\text { direction }\end{array}$ & $\begin{array}{l}\text { - Language and } \\
\text { behavior that } \\
\text { connect, } \\
\text { connote, } \\
\text { imply valuing } \\
\text { and trusting } \\
\text { optimistic } \\
\text { aspirations }\end{array}$ & $\begin{array}{ll}\circ & \begin{array}{l}\text { Positive } \\
\text { reinforce- } \\
\text { ments }\end{array} \\
\circ & \begin{array}{l}\text { Positive } \\
\text { directives }\end{array} \\
\circ & \text { Praises } \\
\circ & \text { Encouraging } \\
& \text { statements } \\
\circ & \text { Competence }\end{array}$ & $\begin{array}{l}\text { Students are seated in table } \\
\text { groups all working on } \\
\text { individual assignments. Some } \\
\text { are writing narratives, some are } \\
\text { doing volcano research } \\
\text { projects, some are coloring, } \\
\text { and others are working on their } \\
\text { laptops. Teacher walks around } \\
\text { and checks in on each table } \\
\text { group. Then she goes back to } \\
\text { the front table where she has } \\
\text { two students working with her } \\
\text { on their narrative essays. } \\
\text {-Let me know if you don't } \\
\text { understand it. } \\
\text {-Are you stuck? } \\
\text {-What part are you at? } \\
\text {-Do you mind if I read this } \\
\text { part? } \\
\text {-Are you doing your } \\
\text { conclusion? Student is not } \\
\text { sure ... Teacher reads his } \\
\text { writing and smiles. } \\
\text {-Wow this is really good! } \\
\text { She engages him in a } \\
\text { conversation. } \\
\text { What is the character going to } \\
\text { do next? He explains and then } \\
\text { she provides the student with }\end{array}$ \\
\hline
\end{tabular}




\begin{tabular}{|c|c|c|c|c|c|c|}
\hline & & & & & & $\begin{array}{l}\text { some direction. } \\
\text {-You have quite the story } \\
\text { here! } \\
\text { (They both laugh) Then the } \\
\text { teacher turns to the next } \\
\text { student. } \\
\text { So what do you put at the end } \\
\text { of a sentence? } \\
\text { What can you use instead of } \\
\text { the word it? Then looks } \\
\text { around and she checks in with } \\
\text { a student across the room.. } \\
\text { Are you ok? Student brings up } \\
\text { her dra wing. She is very proud } \\
\text { of it. Wants to show it off. } \\
\text { Ok, please let's not distract } \\
\text { these two who are working } \\
\text { here. } \\
\text { Teacher goes from one table } \\
\text { group to the next, "Oh, WOW! } \\
\text { I like it! Beautiful colors!" }\end{array}$ \\
\hline & $\begin{array}{l}\text { Optimism } \\
\text { (Freire, 1997) } \\
\text { Ethics (Freire, } \\
\text { 1997) } \\
\text { Criticality } \\
\text { (Freire, 1974, } \\
\text { Giroux, 2011) } \\
\text { Social and } \\
\text { Historical } \\
\text { Consciousness } \\
\text { (Freire, 1974, } \\
\text { 1997) } \\
\text { Humanizing } \\
\text { Practices } \\
\text { (Freire, 1970, } \\
\text { 1974, 1994, } \\
\text { 1997) }\end{array}$ & $\begin{array}{l}\text { Teachers } \\
\text { prepared } \\
\text { written tasks } \\
\text { and } \\
\text { assignments }\end{array}$ & $\begin{array}{l}\text { Teacher } \\
\text { delivery in } \\
\text { prompting } \\
\text { critical } \\
\text { thinking and } \\
\text { aspirations to } \\
\text { be caring } \\
\text { socially } \\
\text { conscious and } \\
\text { ethical. }\end{array}$ & $\begin{array}{l}0 \\
0\end{array}$ & $\begin{array}{l}\text { Higher } \\
\text { levels of } \\
\text { questioning } \\
\text { Making } \\
\text { value } \\
\text { judgments } \\
\text { that show } \\
\text { caring and } \\
\text { ethical } \\
\text { disposition } \\
\text { based upon } \\
\text { the criteria }\end{array}$ & \\
\hline & $\begin{array}{l}\text { Sense of } \\
\text { Community } \\
\text { (hooks, 2003, } \\
\text { Freire, 1997) } \\
\text { Caring } \\
\text { Relationships } \\
\text { (Noddings, } \\
\text { 2017, Freire } \\
\text { 1997) } \\
\text { Knowledge of } \\
\text { Social \& } \\
\text { Cultural } \\
\text { Capital }\end{array}$ & $\begin{array}{l}\text { Teachers } \\
\text { composed } \\
\text { lesson plans }\end{array}$ & $\begin{array}{l}\text { Teacher } \\
\text { planning that } \\
\text { may elicit a } \\
\text { sense of } \\
\text { community } \\
\text { and } \\
\text { humanizing } \\
\text { practices. }\end{array}$ & & $\begin{array}{l}\text { Lesson } \\
\text { plans that } \\
\text { requires } \\
\text { collaboratio } \\
\mathrm{n} \text { and } \\
\text { creativity in } \\
\text { manifesting } \\
\text { voice and } \\
\text { advocacy } \\
\text { that reflect } \\
\text { sense of } \\
\text { community } \\
\text { and }\end{array}$ & \\
\hline
\end{tabular}




\begin{tabular}{|c|c|c|c|}
\hline $\begin{array}{ll} & \text { (Bourdieu, } \\
& 1986) \\
\checkmark & \text { Critical } \\
& \text { Citizenry } \\
& (\text { Giroux, 2011, } \\
& \text { Freire, 1997) } \\
\checkmark & \text { Democratic } \\
& \text { Advocacy } \\
& (\text { Giroux, 2011) } \\
\end{array}$ & & $\begin{array}{l}\text { humanizing } \\
\text { practices }\end{array}$ & \\
\hline & \multicolumn{3}{|c|}{$\begin{array}{l}\text { Other observations: You would never know that his is an SDC/SPED English } \\
\text { class. Students are calm, working in groups, sitting together. Teacher is happy; } \\
\text { students are happy, and engaged. They are proud of their work; the environment is } \\
\text { very comfortable and almost home like. Signs on the wall say, "You Matter," "In this } \\
\text { class we dream big, have fun, laugh, and learn!" There are college banners in the } \\
\text { room, student artwork is up, they painted pictures of the solar system; the teacher is } \\
\text { very pleasant and respectful, which has translated into the students' relationships with } \\
\text { her and with each other. }\end{array}$} \\
\hline
\end{tabular}

